\title{
Looking Wider and Further: \\ The Evolution of Galaxies Inside Galaxy Clusters
}

\author{
by \\ Yuanyuan Zhang \\ A dissertation submitted in partial fulfillment \\ of the requirements for the degree of \\ Doctor of Philosophy \\ (Physics) \\ in the University of Michigan \\ 2016
}

Doctoral Committee:

Professor Timothy A. McKay, Chair

Professor Henriette Elvang

Professor David Gerdes

Professor Dragan Huterer

Professor Christopher J. Miller 
(C) Yuanyuan Zhang

2016 


\section{$\begin{array}{lllllllllllllll}\text { A } & C & K & \mathbf{N} & \text { O } & \text { W } & \text { L } & \text { E } & \text { D } & G & \text { M } & \text { E } & \mathbf{N} & \mathbf{T} & \mathbf{S}\end{array}$}

The road leading to this dissertation was not free from stress or problems, but many people have helped making the journey fulfilling and entertaining. First and Foremost, I want to thank my advisor, Tim McKay, for the training and the guidance. I had no previous experience in astronomy or astrophysics, but Tim took me on without hesitation. Over the years, I learned a great deal from his words and deeds: how to do research, how to write and speak about research results, and how to establish fruitful collaborations. I also feel lucky that Tim helped me raising awareness about work-life balance and being practical while being a perfectionist.

I have been working with Chris Miller a lot since 2013. In the past a couple of years, he treated me as one of his own students and taught me many research skills without any reservation. He was keen on making me to pursue extra research, collaboration and observation opportunities. I would have missed many of them if it wasn't him.

I learned a lot about cosmology theories and simulations from the weekly meetings organized by Gus and Dragan. Gus is alway pressing me for my latest proposals and paper drafts, and offered many insightful feedbacks. Dave and Greg mentored my research with the DES collaboration, and helped to make sure that I communicate with people about my work. Michigan postdocs Joerg and Jeeseon supervised me when I was a junior graduate student. Michigan alumni Adam and Tomasz offered many helpful dicussions while they were here.

The bonds with the DES collaboration and the XCS collaboration (the XMM Cluster Survey) have benefitted me enormously. The feedbacks from the DES cluster working group, the DES galaxy evolution working group and the XCS collaboration are critical in shaping my DES and XCS projects. Many collaborators have generously provided their code and catalogs, or patiently answered my questions about data and simulations. More than twenty people have commented on my paper drafts at different stages of preparation. The telephone discussions within the two collaborations are always inspiring and motivating. This section would become too long if I list the people that have helped on various issues, but at least let me thank Kathy Romer here for supervising my participation in both the DES and the XCS collaborations.

My friends have helped to make the past five years fruitful and unforgettable: Scott, 
Tom, Paul and Arina, Ping, Zhao, Mijin, Arya and many others. I especially want to thank Jia $\mathrm{Xu}$ for taking care of me after an exercise accident.

I am grateful that my family has always stood with me. My brother across the Pacific ocean helped me staying connected to different sides of life. My parents were understanding, cheerful, and eagerly helpful. I've become interested in mathematical and physical science since childhood. However, being a female, it took some extra courage to push it through. My parents were firm that women deserve any kinds of excellence and happiness with any kinds of career choice. This dissertation is dedicated to my mother and father.

The work in this dissertation uses early DES data, which were released internally to the DES collaboration. My collaborators and I deeply appreciate the availability of this high quality data set. We are grateful for the extraordinary contributions of our CTIO colleagues and the DECam Construction, Commissioning and Science Verification teams in achieving the excellent instrument and telescope conditions that have made this work possible. The success of this project also relies critically on the expertise and dedication of the DES Data Management group.

Funding for the DES Projects has been provided by the U.S. Departmtent of Energy, the U.S. National Science Foundation, the Ministry of Science and Education of Spain, the Science and Technology Facilities Council of the United Kingdom, the Higher Education Funding Council for England, the National Center for Supercomputing Applications at the University of Illinois at Urbana-Champaign, the Kavli Institute of Cosmological Physics at the University of Chicago, the Center for Cosmology and Astro-Particle Physics at the Ohio State University, the Mitchell Institute for Fundamental Physics and Astronomy at Texas A\&M University, Financiadora de Estudos e Projetos, Fundação Carlos Chagas Filho de Amparo à Pesquisa do Estado do Rio de Janeiro, Conselho Nacional de Desenvolvimento Científico e Tecnológico and the Ministério da Ciência, Tecnologia e Inovação, the Deutsche Forschungsgemeinschaft and the Collaborating Institutions in the Dark Energy Survey.

Collaborating Institutions are Argonne National Laboratory, the University of California at Santa Cruz, the University of Cambridge, Centro de Investigaciones Energéticas, Medioambientales y Tecnológicas-Madrid, the University of Chicago, University College London, the DES-Brazil Consortium, the University of Edinburgh, the Eidgenössische Technische Hochschule (ETH) Zürich, Fermi National Accelerator Laboratory, the University of Illinois at Urbana-Champaign, the Institut de Ciències de l'Espai (IEEC/CSIC), the Institut de Física d'Altes Energies, Lawrence Berkeley National Laboratory, the LudwigMaximilians Universität München and the associated Excellence Cluster Universe, the University of Michigan, the National Optical Astronomy Observatory, the University of 
Nottingham, The Ohio State University, the University of Pennsylvania, the University of Portsmouth, SLAC National Accelerator Laboratory, Stanford University, the University of Sussex, and Texas A\&M University.

The work in this dissertation also uses data from the SDSS project. Funding for the SDSS and SDSS-II has been provided by the Alfred P. Sloan Foundation, the Participating Institutions, the National Science Foundation, the U.S. Department of Energy, the National Aeronautics and Space Administration, the Japanese Monbukagakusho, the Max Planck Society, and the Higher Education Funding Council for England. The SDSS Web Site is http://www.sdss.org/.

The SDSS is managed by the Astrophysical Research Consortium for the Participating Institutions. The Participating Institutions are the American Museum of Natural History, Astrophysical Institute Potsdam, University of Basel, University of Cambridge, Case Western Reserve University, University of Chicago, Drexel University, Fermilab, the Institute for Advanced Study, the Japan Participation Group, Johns Hopkins University, the Joint Institute for Nuclear Astrophysics, the Kavli Institute for Particle Astrophysics and Cosmology, the Korean Scientist Group, the Chinese Academy of Sciences (LAMOST), Los Alamos National Laboratory, the Max-Planck-Institute for Astronomy (MPIA), the MaxPlanck-Institute for Astrophysics (MPA), New Mexico State University, Ohio State University, University of Pittsburgh, University of Portsmouth, Princeton University, the United States Naval Observatory, and the University of Washington.

The Millennium Simulation databases used in this dissertation and the web application providing online access to them were constructed as part of the activities of the German Astrophysical Virtual Observatory (GAVO). 


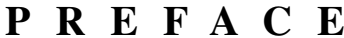

Galaxy clusters are important subjects of study for both cosmology and astrophysics. Thanks to the enormous amount of twinkling stars in their member galaxies, clusters are easy to recognize in optical images. This dissertation focuses on these galaxies, the cluster component that shines in visible light.

The work reported here includes four studies which I have led over the last five years. Three have already been published as first-author papers. The study presented in Chapter 2 is published on the Publications of the Astronomical Society of the Pacific under the title Crowded Cluster Cores: An Algorithm for Deblending in Dark Energy Survey Images. The study presented in Chapter 3 is published on the Astrophysical Journal under the title Galaxies in X-Ray Selected Clusters and Groups in Dark Energy Survey Data. I. Stellar Mass Growth of Bright Central Galaxies Since $z \sim 1.2$. Chapter 5 is published on the Astrophysical Journal with the title Studying Intercluster Galaxy Filaments through Stacking gmBCG Galaxy Cluster Pairs. The work presented in Chapter 4 is intended for submission to the the Astrophysical Journal with the working title Galaxies in X-ray Selected Clusters and Groups in Dark Energy Survey Data II: Luminosity Function of red sequence cluster galaxies at $0.1<z<1.05$. 


\section{TABLE OF CONTENTS}

Acknowledgments $\ldots \ldots \ldots \ldots \ldots \ldots \ldots \ldots \ldots$ ii

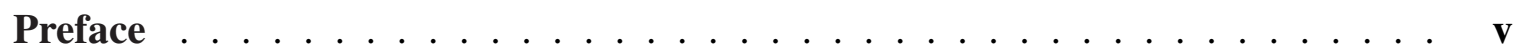

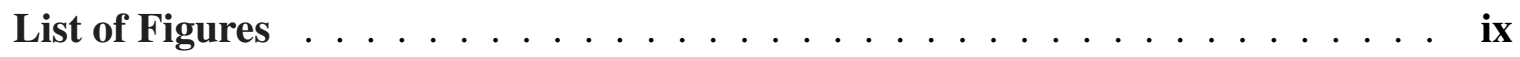

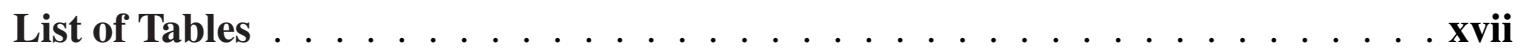

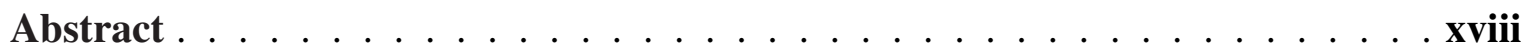

\section{Chapter}

1 Introduction . . . . . . . . . . . . . . . . . . . . . 1

1.1 Cluster Content . . . . . . . . . . . . . . . . . . 2

1.2 Multi-Wavelength Data . . . . . . . . . . . . . . . . 4

1.3 Wide Field Surveys $\ldots \ldots \ldots \ldots$

1.4 Dissertation Outline . . . . . . . . . . . . . . . . . 6

2 Detecting Cluster Galaxies . . . . . . . . . . . . . . . . . . . 8

2.1 Topic Introduction . . . . . . . . . . . . . . . . . . . . . . . . 8

2.2 Software Features and Functions $\ldots \ldots \ldots$

2.3 Software Algorithms . . . . . . . . . . . . . . . . 12

2.3.1 Source Detection . . . . . . . . . . . . . . . . . . 12

2.3.2 Deblend Blended Sources _. . . . . . . . . . . . . . 18

2.4 Methods Validation . . . . . . . . . . . . . . . . . 21

2.4.1 Photometry Measurement . . . . . . . . . . . . 25

2.4 .2 Purity and Completeness . . . . . . . . . . . . . . 25

2.5 Discussion . . . . . . . . . . . . . . . . . . . . . . . . 29

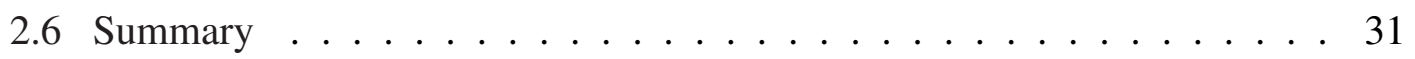

3 Evolution of Cluster Central Galaxies . . . . . . . . . . . . . . 33

3.1 Topic Introduction . . . . . . . . . . . . . . . . . . . . . . 33

3.2 Data . . . . . . . . . . . . . . . . . 36

3.2.1 DES Science Verification Data . . . . . . . . . . . . . . 36

3.2.2 The DES-XCS Cluster and Group Sample . . . . . . . . . . . . 36

3.2 .3 BCG Selection . . . . . . . . . . . . . . . . . . . 39

3.3 Galaxy Cluster Mass . . . . . . . . . . . . . . . . . . . . . . . 39

3.3.1 Cluster Mass From X-ray Temperature _ . . . . . . . . . . 39 
3.3.2 Cluster mass from X-ray Luminosity . . . . . . . . . . . . . 42

3.3.3 Mass Uncertainties and the Choice between Temperature and Luminosity Based Masses . . . . . . . . . . . . . . . . . . . 42

3.4 BCG Photometry . . . . . . . . . . . . . . . . . . . . . 42

3.4.1 Petrosian Magnitude . . . . . . . . . . . . . . . . . 45

3.4 .2 Kron Magnitude . . . . . . . . . . . . . . . . . . 45

3.4.3 Profile Fitting Magnitude . . . . . . . . . . . . . . . . . 46

3.4.4 Aperture Magnitude Used in this Work . . . . . . . . . . . . 47

3.5 BCG Luminosity and Stellar Mass . . . . . . . . . . . . . . . . . 48

3.5.1 BCG Luminosity Uncertainty . . . . . . . . . . . . . . . . 49

3.5.2 BCG Mass-to-Light Ratio Uncertainty . . . . . . . . . . . . . . . 49

3.6 Simulation Matching Analysis . . . . . . . . . . . . . . . 51

3.6.1 Simulation Sample Selection . . . . . . . . . . . . . . . . 54

3.6.2 Redshift Evolution of the Observed BCGs . . . . . . . . . . . . . 55

3.6.3 High Redshift BCGs . . . . . . . . . . . . . . . . . . 56

3.7 BCG-Cluster Mass Relation . . . . . . . . . . . . . . . . 56

3.7.1 Modeling the BCG-Cluster Mass Relation . . . . . . . . . . 56

3.7.2 Additional Information on Covariance and the Likelihood Function 59

3.7.3 Constraints on the BCG-Cluster Mass Relation . . . . . . . . . 60

3.8 BCG Stellar Mass Growth since $z \sim 1.0 \ldots \ldots$. . . . . . . . . 62

3.8 .1 Method and Test . . . . . . . . . . . . . . . . 62

3.8.2 Growth Rate from $z=1.0$ to $z=0$. . . . . . . . . . . 64

3.8 .3 Role of ICL Production . . . . . . . . . . . . . . 65

3.9 Summary and Discussion . . . . . . . . . . . . . . . . . 67

4 Evolution of Cluster Red Sequence Galaxies … . . . . . . . . . . . . 69

4.1 Topic Introduction . . . . . . . . . . . . . . . . . . . . . . . . . . 69

4.2 Data . . . . . . . . . . . . . . . . . . . . 71

4.2.1 Dark Energy Survey Science Verification Data . . . . . . . . 71

4.2.2 The XCS-SV cluster sample . . . . . . . . . . . . . . 71

4.2.3 The DES Photometric Data . . . . . . . . . . . . . . . 73

4.3 Red Sequence Galaxy Selection . . . . . . . . . . . . . . . . . . 74

4.3.1 Window Color Selection . . . . . . . . . . . . . . 74

4.3.2 RedMaPPer Member Selection . . . . . . . . . . . . . . . . 76

4.4 Completeness Function . . . . . . . . . . . . . . . . . . 76

4.4.1 The Completeness Function Model . . . . . . . . . . . . . 76

4.4.2 Relations between Model parameters and Image Depth . . . . . 78

4.4.3 Magnitude Limits of the LF Analyses . . . . . . . . . . . . . 79

4.5 Methods . . . . . . . . . . . . . . . . . . . 82

4.5.1 The histogram method . . . . . . . . . . . . . 82

4.5.2 The Bayesian Inference methods . . . . . . . . . . . . 85

4.5.3 redMaPPer Selected objects . . . . . . . . . . . . . . . . . . 89

4.6 Results . . . . . . . . . . . . . . . . . . . . . . . . . 91

4.6.1 Results in Redshift/Mass Bins . . . . . . . . . . . . . . . . 91

4.6.2 Results from the Bayes Dependence Model . . . . . . . . . . 92 
4.7 Discussion . . . . . . . . . . . . . . . . . . . . . 96

4.7.1 Comparison to Literature . . . . . . . . . . . . . . . . . . 96

4.7.2 The Methods . . . . . . . . . . . . . . . . . . . . 97

4.7.3 Implication on Cluster Red Sequence Formation . . . . . . . . 99

4.8 Summary . . . . . . . . . . . . . . . . . . . . . 100

5 Cluster Ambience - Galaxy Filaments _ . . . . . . . . . . . . . 101

5.1 Topic Introduction . . . . . . . . . . . . . . . . . . . . . 101

5.2 Methods . . . . . . . . . . . . . . . . . . . . . . . 104

5.2 .1 Data . . . . . . . . . . . . . . . . . . . . . . . 104

5.2 .2 Algorithm . . . . . . . . . . . . . . . . . . . . 104

5.2.3 Foreground/Background Subtraction and Filament Detection Significance . . . . . . . . . . . . . . . . . 111

5.2.4 Justification on Using C1/C2 for Foreground/Background Subtraction . . . . . . . . . . . . . . . . . 117

5.2.5 Null Test with Random Cluster Pair Re-Positioning . . . . . . . . 119

5.3 Results . . . . . . . . . . . . . . . . . . . . 120

5.3.1 Color Distributions . . . . . . . . . . . . . . . . 120

5.3.2 Luminosity Function . . . . . . . . . . . . . . . . . . 123

5.3.3 Cluster Richness Dependence . . . . . . . . . . . . . . . . . . 128

5.3 .4 Spatial Distribution . . . . . . . . . . . . . . . . . . . 129

5.4 Detection of Filaments in Simulation Data . . . . . . . . . . . . . 129

5.5 Summary and Discussion . . . . . . . . . . . . . . . 135

5.5 .1 Color Evolution . . . . . . . . . . . . . . . . . 136

5.5 .2 Luminosity Function . . . . . . . . . . . . . . . . 137

5.5.3 Filament Richness and Geometry . . . . . . . . . . . . 138

6 Closing Remarks . . . . . . . . . . . . . . . . . . . . . . . 139

6.1 Recapitulation . . . . . . . . . . . . . . . . . . . . 139

6.2 Outlook . . . . . . . . . . . . . . . . . . . . 142

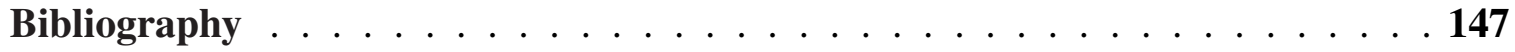




\section{LIST OF FIGURES}

\section{Figure}

2.1 Upper Left: the linear combination of DES $r, i$ and $z$ coadd images for a cluster field. There is a bright star on the left and a brightest cluster galaxy on the right. Upper Right: same image with detected sources marked out. Blue circles are SExtractor detections (226 objects) using standard DES pipeline settings. Red squares (42 detections) are additional detections found through GAIN. Lower left: SExtractor detections with an aggressive deblending setting (DEBLEND_MINCONT $=1 \times 10^{-6}$ ), which tends to introduce many spurious detections. Lower Right: black circles (80 detections) are the additional Sextractor detections gained by more aggressive deblending. For comparison, GAIN detections from the upper right panel are shown again in this figure. In these figures, we only show sources brighter than 24.0 mag in $i$. . . 10

2.2 Left: $r$ band DES coadd images of four blended sources. The circles indicate the areas to be deblended. Middle: images that contain light from neighbors of the blended sources. Right: the residual between the left and middle panels, which shows the light from the blended sources within the circle. . . . . . . 11

2.3 A flow chart demonstrating the GAIN computing steps explained in Section $2.3 . \ldots \ldots \ldots \ldots \ldots \ldots$

2.4 Upper left: Original DES coadd image, same as the one shown in Figure 2.1, but is zoomed in to best illustrate the segmentation procedure. Upper right: the segmentation map derived using all the local intensity maxima. Lower left: the segmentation map derived using all the local maxima of the weighted Laplacian map. Lower right: the segmentation map derived using the cross matched local maxima between the intensity map and the weighted Laplacian map. In the three segmentation maps, the empty white regions are the "boundarys" (see Section 2.3.1.1 for definition), and the pixels in black are associated with the seeds (local maxima from the intensity map, from the weighted laplacian map, and the cross matching between the two). . . . . . . . . . . . . . . 14

2.5 Upper Left: original DES coadd image, same as the one shown in Figure 2.1 and Figure 2.4. Upper Right: the Laplacian map of the same image. The fine features of the original image, like the spiral arms of the galaxy at the bottom right, are enhanced in the Laplacian map. Lower Left: intensity weighting map $\left(I_{w}\right)$ derived as described in Section 2.3.1.2. High intensity peaks are suppressed in this weighting map. Lower right: weighted Laplacian map in Section 2.3.1.2 which combines the Laplacian map with the intensity weighting map to bring out faint features. . . . . . . . . . . . . 16 
2.6 Left: An $r$ band DES coadd image. The 10 sigma limiting magnitude of this image is $25.3 \mathrm{mag}$ as measured from the SExtractor mag_auto uncertainty. Right: The same image after adding simulated BCGs. The size of these two images is approximately $3.15^{\prime} \times 3.15^{\prime}$. The apparent magnitude of the simulated BCGs is approximately $\sim 19$ mag. . . . . . . . . . . . . . . . . 21

2.7 Comparison of photometry measurements and star/galaxy separation quantities with SExtractor using the "global background" setting. (a)(b) Offsets between the "truth" magnitudes and measurements from altered images for "artificially" blended objects. (c)(d) Comparison of star/galaxy separation quantities for "artificially" blended objects. . . . . . . . . . . . . . . .

2.8 Comparison of photometry measurements and star/galaxy separation quantities with SExtractor using the "local background" setting. (a)(b) Offsets between "truth" magnitudes and measurements from altered images for "artificially" blended objects. (c)(d) Comparison of star/galaxy separation quantities for "artificially" blended objects. . . . . . . . . . . . . . . . . . .

2.9 Completeness of the $\mathrm{C} 1$ catalog (blue solid line, from SExtractor) and completeness of the combination (red dashed line) of $\mathrm{C} 1$ and $\mathrm{C} 2$ (C2 from GAIN) as computed in Section 2.4.2.1. The non-negligible incompleteness of SExtractor catalog can be improved by GAIN all the way to $25.5 \mathrm{mag}$. The vertical dotted line shows the 10 sigma limiting magnitude of the image that the test is performed on. The errors in this plot are estimated assuming poisson distribution. . . . . . . . . . . . . . . 26

2.10 Purity of C1 (blue Solid line) by SExtractor and C2 (red dotted line) by GAIN and purity of the combination of $\mathrm{C} 1$ and $\mathrm{C} 2$ (red dashed line) as computed in Section 2.4.2.2. The unsatisfying purity of $\mathrm{C} 1$ at the bright end indicates that the deblending procedure of SExtractor is prone to introduce spurious detections. Also, the purity of the combination of $\mathrm{C} 1$ and $\mathrm{C} 2$ is affected by the purity of $\mathrm{C} 1$. On the other hand, the sources contained in C2 (GAIN output) are highly consistent with the previous running. The vertical dotted line marks the 10 sigma limiting magnitude of the image that the test is performed on. The errors in this plot are estimated assuming poisson distribution. . . . . . . .

3.1 The mass and redshift distribution of the DES-XCS sample (black stars) compared to that of Lidman et al. (2012, red circles). . . . . . . . . . . . . .

3.2 Distances between the BCGs and the X-ray emission centers for our cluster sample. Half of the BCGs are separated less than $0.07 \mathrm{Mpc}$ (transverse comoving distance with negligible uncertainties from redshifts measurements) from the X-ray centers, and the large separations $(>0.4 \mathrm{Mpc})$ happen in clusters that may not be relaxed or appear to have spurious foreground/background emissions. . . . . . . . . . . . . . . . .

3.3 Comparison of the cluster masses derived from a few $M-T$ scaling relations. We plot the mass derived from the Kettula et al. 2013 relation on the $x$-axis of all the panels. The Kettula et al. $2013 M-T$ scaling relation agrees well with other relations at the cluster scale (See Section 3.3.1). For simplification, we only include X-ray temperature measurement uncertainty in this figure. . . 
3.4 Left: We derive cluster masses from both X-ray luminosities and X-ray temperatures, and decide which one to use through comparing their uncertainties. Right: X-ray temperature and X-ray luminosity of the XCS clusters plotted against a few scaling relations in literature.The grey band shows the redshiftdependent $L-T$ relation in Hilton et al. 2012 between $\mathrm{z}=1$ and $\mathrm{z}=0$. . . . .

3.5 We investigate measurement bias associated with Kron magnitude and aperture magnitude using the UFIG sky simulation (see Section 3.4.4 for details). Note that this test is done for a general galaxy population rather than BCGs. BCGs below redshift 1.0 generally have Sersic index $>2$ and apparent magnitude below 22. In the top row, $m_{\text {true }}$ is the galaxy's input total magnitude, but in the bottom row, $m_{\text {true }}$ is the galaxy's input $32 \mathrm{kpc}$ aperture magnitude. To summarize this figure, Kron magnitude tend to under-estimate the brightness of bulge-like galaxies and extended galaxies, while aperture magnitude remain well-behaved for galaxies of all profiles and sizes. The measurements from both systems do become biased for faint galaxies with apparent magnitude above 23, but the bias is un-important for this work. For efficiency, we use SExtractor output in this comparison, which matches our own measurements for a general galaxy population. . . . . . . . . . . . . .

3.6 The popular SExtractor software tends to under-estimate BCG Kron radius, resulting in significant brightness under-estimation. In panel (b), we show the difference in Kron magnitude measurement, $\Delta m$, when the measurement is

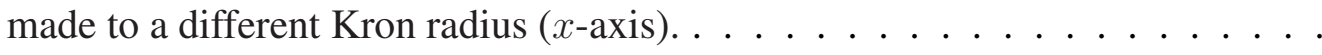

3.7 (a): Measurement dispersion of aperture magnitude, profile fitting magnitude and Kron magnitude. (b): We sample BCG apparent magnitude from multiple exposures to evaluate the measurement uncertainty, and our measurement accuracy is limited by the number of exposures we have. . . . . . . . . .

3.8 (a) We apply our stellar model fitting procedure to the DL07 simulation BCGs using their simulated SDSS $g, r, i$, $z$ photometry. Because the fitting procedure and the simulated BCG photometry are based on different SSP models and IMFs, there exists a 0.05 dex systematic offset. The derived mass-to-light ratio also has statistical uncertainty of $\sim 0.05$ dex at $z=0$ and $\sim 0.1$ dex at $z=1$. (b) We evaluate the stellar mass uncertainty combining redshift, magnitude and mass-to-light ratio uncertainties. The stellar mass uncertainty is dominated by apparent magnitude uncertainty at $z<0.8$, and redshift uncertainty at $z>0.8$.

3.9 Mass and redshift distribution of the cluster sample used for this work and distribution of the simulation clusters drawn from DL07. The black data points show the masses and mass uncertainties of the XCS clusters. The red data points show the median masses and the 0.158 and 0.842 percentiles of the simulation clusters. For clarification, we show the mass distribution of the resampled DL07 clusters at the redshift of the corresponding XCS cluster with a small offset. 
3.10 Comparison between the observed and simulated BCG properties. We show the median and $0.158,0.842$ percentiles of the differences. The dots are data points outside the range of the uncertainty whiskers, and the red diamonds are moving medians. The blue bands show the fitted linear model with dependence on look-back time, encompassing $1 \sigma$ uncertainties. We notice that the observed BCGs are becoming under-massive/under-luminous at decreasing redshift. Note that we have not included stellar mass or luminosity measurement uncertainties in the linear fit. We also use a 95\% confidence interval clipping technique to remove outliers. . . . . . . . . . . .

3.11 Posterior distribution of the parameters, $\log m_{0}, \alpha, \beta$ and $\epsilon$, in the BCG-cluster mass relation (Equation 3.1), based on the BCG stellar mass derived in $32 \mathrm{kpc}$ aperture. The histogram in each column shows the marginalized distribution of the corresponding parameter. Other panels show the correlation between two parameters noted on the $\mathrm{x}$ and $\mathrm{y}$ axes. . . . . . . . . . . . 57

3.12 To derive BCG stellar mass growth rate from BCG-cluster mass relations, we will need to derive cluster mass growth history from simulations. In the top panel, we show the halo mass evolution history of $\sim 200$ halos with $\log M_{200}=$ 13.8 at $z=1.0$. We utilize Equation 3.11 (we are using the BCG-cluster mass relation in DL07) to derive the BCG stellar mass growth rate shown by the red dashed line. In the bottom panel, we show the test result for this method (see Section 3.8.1 for details). Overall, our approach well reproduces the average growth rate within $1 \sigma$ for simulation BCGs. . . . . . . . . . . . .

3.13 This plot shows our BCG stellar mass growth estimation with the full sample
and with two mass-limited sub-samples. We also show the measurements in Lidman et al. 2012 and Lin et al. 2013 and the BCG growth rate in the DL07 simulation. Our estimation is consistent with previous measurements, but slower than DL07 by $\sim 2.5 \sigma$. The uncertainty from DL07 is extremely small as the simulation is well sampled. . . . . . . . . . . .

3.14 We show BCG growth rates from toy models adding more rapid ICL production or more dramatic BCG star formation to the DL07 simulation. Including extra constant ICL production at $20-40 M_{\odot} /$ yr well reproduces our observed growth rate. We also show the predicted $\mathrm{BCG}$ growth rate from Contini et al. 2014, which has updated the DL07 simulation with more realistic ICL production. The BCG growth rate in Contini et al. 2014 agrees well with our measurement. ...................... 66

4.1 The redshifts, masses, and mass uncertainties of the XCS-SV clusters. Vertical solid lines and Horizontal dashed lines show the ranges of the subsamples used to constrain LF mass dependence and redshift evolution respectively. . . . . . 72 
4.2 We select red sequence galaxies with $g-r$ color at $z<0.5$, and $r-i$ color at $z>0.5$. Having chosen the clipping width in $g-r$ to be $0.2 \mathrm{mag}$, we adjust the clipping width in $r-i$ to match the number of selected cluster galaxies. This figure shows how the clipping width in $r-i$ changes cluster galaxy counts in comparison to selecting with $g-r$. See Section 4.3 for details. Different symbols (circle, square and triangle) indicates cluster galaxy counts to different magnitude limits. We choose the clipping width in $r-i$ to be 0.13 (vertical dashed lines) according to this test. . . . . . . . . . . . . 75

4.3 This figure demonstrates our procedure on evaluating the completeness function with the UFIGsimulation. We use a completeness function to model the difference between the observed magnitude distribution (green squares in top panel) of observed objects and the truth magnitude distribution of all truth objects (black circles in top panel). Given a truth object of truth magnitude $m$, we model the probability of observing an object with observed magnitude $m$ (green squares in lower panel), which is well described by a complementary error function. For comparison, we also show the truth magnitude distribution of the observed objects (red triangles) . . . . . . . . . . . . . . . . 77

4.4 This figures shows the relations between completeness function parameters and image depth, characterized by $10 \sigma$ limiting magnitude. The relations derived from the UFIG simulation generally agree with those from the BALROG simulation. The $m_{50}$ values evaluated from re-stacking deep supernovae data appear to be 0.1-0.2 mag deeper than the relations derived from sky simulations, but the differences can be explained by Kron magnitude bias shown in Z15. Therefore, we use the relations from the UFIG simulation to model completeness function in this chapter. . . . . . . . . . . . . . 78

4.5 We evaluate the $m_{50}$ parameter for cluster and field regions of the same depth with the BALROG simulation. There appears to be a trend that the $m_{50}$ of the cluster regions are shallower by $\sim 0.1 \mathrm{mag}$, although the result is not well constrained due to small sample size. . . . . . . . . . . . . . . 80

4.6 For each cluster, we derive a completeness magnitude limit, $m_{\text {lim }}$ from the completeness function. Most of the $z<0.4$ clusters are complete to $m_{z}^{*}+$ 2 mag and beyond. This is also true for more than $2 / 3$ of the clusters at $z>0.4 .81$

4.7 Cluster red sequence lumnosity function derived in redshift bins for the window color selection (black filled circles) and redMaPPer member selection (red squares). The left column shows cluster galaxy magnitude distribution at linear scale. The right column shows the same data but at log scale. We also plot the Schechter function fitting results for the window color selected galaxies (black solid and dashed lines in the left column) and for the redMapper selected members (red solid and dashed lines in the right column). . . . . . . 83 
4.8 Cluster red sequence lumnosity function derived in $M_{200}$ bins for the window color selection (black filled circles) and redMaPPer member selection (red squares). The left column shows cluster galaxy magnitude distribution at linear scale. The right column shows the same data but at log scale. We also plot the Schechter function fitting results for the window color selected galaxies (black solid and dashed lines in the left column) and for the redMapper selected members (red solid and dashed lines in the right column). . . . . . . . 84

4.9 Redshift evolution of the faint end slope, $\alpha$, and the characteristic magnitude, $m^{*}$, derived with four different methods. . . . . . . . . . . . . . 90

4.10 Mass dependence of the faint end slope, $\alpha$, and the characteristic magnitude, $m^{*}$, derived with four different methods. . . . . . . . . . . . . . . . . . 91

4.11 Constraints on the amplitude of the luminosity function for individual clusters. We do not observe significant redshift evolution effect of the $\phi^{*}-M_{200}$ relations, therefore we only model it as mass dependent in this work. The linear relation between $\phi^{*}$ and $M_{200}$ is determined at the same time in the Bayes dependence method (Section4.5.2.3). . . . . . . . . . . . . . . . . . 94

4.12 In the Bayesian dependence model, we probe cluster mass using priors from $\mathrm{X}$-ray measurements. The constrained cluster masses generally agree with $\mathrm{X}$-ray measurements. The mass uncertainty improves when the X-ray measurement uncertainty, $M_{\mathrm{obs}}$, is larger than 0.3 dex. . . . . . . . . . . 95

4.13 Comparison to literature on the redshift evolution of the faint end slope, $\alpha$, and the characteristic magnitude $m^{*}$. . . . . . . . . . . . 95

4.14 Comparison to literature on the mass dependence of the faint end slope, $\alpha$, and the characteristic magnitude, $m^{*}$. . . . . . . . . . . . . 96

4.15 Simulation test of the Bayes method. The dashed lines show the truth input values of $\alpha$ and $m_{*}$. The black filled circles and red filled squares show the constraints derived with samples that match the sizes of our current data and future data release respectively. $\ldots \ldots \ldots \ldots \ldots$

5.1 Flowchart illustration of the algorithm described in Section 5.2.2. The left side of the figure illustrates the manipulations on galaxy data in order to acquire stacked cluster pair galaxy fields. The right side illustrates the the manipulations on the random point catalog in order to acquire the sky coverage weighting, which tells how many times each pixel in the galaxy stacking is covered by real sky data. The manipulations on random points are identical to the manipulations on real galaxy data before "Compute the Weighting" step (See Section 5.2.2.5 for details). Arrows indicate the processing flow. . . . . . 103

5.2 Cluster pairs' projected separation on the plane of the sky at their median redshift, $r_{\text {projected }}$, versus their 3-d physical separation, $r_{3 \mathrm{D}}$. The white dashed

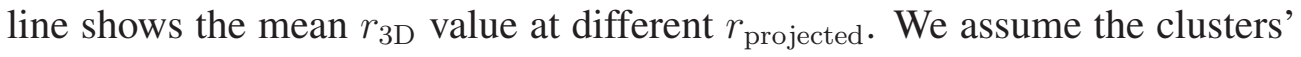
photometric redshift are purely due to Hubble flow and use the law of cosine for calculating $r_{3 \mathrm{D}}$. The cluster pairs' projected separation on the plane of the sky is cut off at $\sim 35 h^{-1} \mathrm{Mpc}$, depending on the exact redshift of the projection, but their $3-\mathrm{d}$ separation can be as large as $90 h^{-1} \mathrm{Mpc}$. . . . . . . . 105 
5.3 (a) Shows a galaxy overdensity significance contour map from stacking galaxy sets of clusters at $0.14<z<0.18$. There is a significant overdensity in the middle of the map, caused by the presence of many cluster galaxies. (b) Shows null test results from stacking randomly re-positioned pairs, where the whole field is noisily flat. In (a) and (b), the solid line and dashed line boxes mark out the four regions defined in Section 5.2.3. . . . . . . . . . . . . . 112

5.4 Projected galaxy number density in the filament region (FL) and the comparison 1 region $(\mathrm{C} 1)$ at $0.14<z<0.18$ when stacking real cluster pairs or when performing null test with randomly re-positioned pairs (see Section 5.2.5 for details). The histograms shows distributions of 40 bootstrapped resamplings of the original stacking. When stacking galaxy sets of real cluster pairs, the FL region (red dashed line) displays a galaxy overdensity above the $\mathrm{C} 1$ region (blue solid line) with a significance of $\sim 5 \sigma$. Galaxy number counts of FL and $\mathrm{C} 1$ at other redshift slices also show similar high significance detection. In null test of stacking randomly re-positioned pairs, we don't observe any overdensity in F1 (blue dotted line) over $\mathrm{C} 1$ (red dash dot line). Because the galaxy overdensity in cluster fields, the galaxy count in F1 and $\mathrm{C} 1$ from stacking real cluster pairs are higher than stacking randomly re-positioned pairs. . . . . . . . 113

5.5 How we get the "pure" color distributions of filament galaxies and cluster galaxies. (a) shows the color distributions of the FL region and the $\mathrm{C} 1$ region, which are almost indistinguishable. After subtracting the $\mathrm{C} 1$ region distribution from the FL region distribution, we obtain the color distribution of pure filament galaxies, which is shown in (b). Subtraction between the CL region and the $\mathrm{C} 2$ region is also applied to get the pure color distribution of cluster galaxies. (c) shows the color distributions of the CL region galaxies and the C2 region, and (d) shows the pure color distribution of cluster galaxies after subtraction. These plots are based on stacking galaxy sets of clusters in the redshift bin $[0.14,0.18]$. Unless otherwise noted, the error bars in this figure and the rest of the chapter are single standard deviation errors estimated from bootstrapping the galaxy sets being stacked. Because one cluster on average appears 5.7 times in the stack (158,897 cluster pairs versus 55,424 clusters), and the error for clusters tend to be underestimated, we enlarge the error estimation of clusters by a factor of $\sqrt{5.7}$ through out the chapter. . . . . . . . . . 114

5.6 (a) Distribution of the number of pair connections each cluster have in the cluster pair catalog. Most clusters are connected to more than 5 other clusters. (b) Distribution of the number of cluster pair connections in the $\mathrm{C} 1$ region (dashed line) and the FL region (solid line). There is no cluster pair connection in $\mathrm{C} 1$ for $30 \%$ of the time, and the average number of cluster pair connections in $\mathrm{C} 1$ is lower than that in FL. Meanwhile, the FL region always contain at least one cluster pair connection since one cluster pair is always aligned along $y=0$ at the FL side. (c) Cumulative probability distribution of the difference between number of cluster pair connections in FL and C1. C1 is unlikely to have equal or more cluster pairs than FL $(\sim 35 \%)$. The horizontal and vertical dotted lines indicate where number of cluster pair connections in FL and C1 are equal. . . . . . . . . . . . . . . . . 118 
5.7 $g-r$ distributions of clusters (a) and filaments (b) in 8 redshift bins. Filaments are bimodal with their color distribution and contain a larger blue galaxy bump than clusters. Also, the filament color distribution shows evidence of redshift evolution in the form of a blue galaxy population that becomes increasingly important at higher redshift. The vertical solid lines in these plots mark the blue/red galaxy color cut used in Section 5.3.1.1. Note that the galaxy population sampled here is somewhat incomplete at $0.38<z<0.42 . \quad \ldots \ldots \ldots 121$

5.8 Redshift evolution of blue galaxy fraction in clusters (a) and filaments (b). These two figures show box plots of the blue galaxy fraction evaluation in clusters and filaments of 40 bootstrapped stacks at each redshift bin. The bottom and top of the boxes represent the lower and upper quartiles, and the whiskers represent the minimum and maximum values of the data, or 1.5 times the quartiles. Data points outside this range are plotted as open circles. We also fit the blue galaxy fraction linearly to the redshift (dashed lines). The blue galaxy population in clusters and filaments both seems to increase at higher redshift, but the change in filaments is less dramatic. Also note that the observed galaxy population is somewhat incomplete at $0.38<z<0.42$ (see discussion in Section 5.2.2.6). . . . . . . . . . . . . . . . . . . . 122

5.9 Luminosity Distributions of filaments (1st and 3rd rows) and clusters (2nd and 4th rows) and fits to the Schechter function (dashed lines) using the MPFIT package. The fitting parameters are listed in Table 5.1. We do not fit for the $M_{r}-5 \log _{10} h<-22.3$ bright end of the luminosity distributions since a LRG population component is possibly present, and it luminosity distribution tend to deviate from the Schechter function. . . . . . . . . . . . . . . 125

5.10 The 2-d projected galaxy number density of filaments, $\Sigma_{\text {pff }}$, with FL and C1 boxes placed at different extension angles. The exact definition of FL and $\mathrm{C} 1$ are listed in Table 5.3. Filament galaxies still can be seen at increasing angles off the intercluster pair axis, but eventually die out when the extension angle from this axis is larger than $45^{\circ} \ldots \ldots \ldots \ldots$. . . . . . . . . . 129

$5.11 g-r$ distributions of filaments at different redshift bins in simulation (a) and SDSS (b). Comparing to SDSS data, the simulation is over-populated with red galaxies. Note that the galaxy population is incomplete at $0.38<z<0.42$ (See discussion in Section 5.2.2.6) and also (b) differs from Figure 5.7 (b) because of the different luminosity cut applied. . . . . . . . . . . . . . 132

5.12 Luminosity distributions of filament galaxies in simulation (1st and 3rd rows) and SDSS (2nd and 4th rows) and their Schechter function fits (dotted lines).The Schechter function fit has a fixed faint end slope, and the rest of the fitting parameters are listed in Table 5.4. The characteristic magnitude of the fitted Schechter function is brighter in simulation than in SDSS, indicating overpopulation of bright galaxies in the simulation. . . . . . . . . . . 133 


\section{LIST OF TABLES}

\section{Table}

3.1 Parameters of the stellar population models . . . . . . . . . . . . . . 48

3.2 Constraints on $\log m_{0}, \alpha, \beta$ and $\sigma$ of the BCG-cluster mass relation (Equation 3.1) and $p$ from the outlier pruning procedure $\ldots \ldots . \ldots 5$

4.1 Constraints on the $\alpha$ and $m *_{z=0.4}$ relations . . . . . . . . . . . . 93

5.1 Fitted Luminosity Function Parameters . . . . . . . . . . . . . . . . . . . 124

$5.2 \Sigma_{\mathrm{pfl}}\left[\left(h^{-1} \mathrm{Mpc}\right)^{-2}\right]$ versus richness at two redshift ranges $\ldots \ldots \ldots . \ldots 128$

5.3 Definitions of the FL and $\mathrm{C} 1$ boxes used in Section . . . . . . . . . . . . . 130

5.4 Fitted Luminosity Function Parameters in Simulation and SDSS . . . . . . . . 134

5.5 SDSS r-band characteristic magnitude measurements . . . . . . . . . . 137 


\begin{abstract}
Galaxy clusters are rare objects in the universe, but on-going wide field optical surveys are identifying many thousands of them to redshift 1.0 and beyond. Using early data from the Dark Energy Survey (DES) and publicly released data from the Sloan Digital Sky Survey (SDSS), this dissertation explores the evolution of cluster galaxies in the redshift range from 0 to 1.0. As it is common for deep wide field sky surveys like DES to struggle with galaxy detection efficiency at cluster core, the first component of this dissertation describes an efficient package that helps resolving the issue. The second part focuses on the formation of cluster galaxies. The study quantifies the growth of cluster bright central galaxies (BCGs), and argues for the importance of merging and intra-cluster light production during BCG evolution. An analysis of cluster red sequence galaxy luminosity function is also performed, demonstrating that the abundance of these galaxies is mildly dependent on cluster mass and redshift. The last component of the dissertation characterizes the properties of galaxy filaments to help understanding cluster environments.
\end{abstract}




\section{CHAPTER 1}

\section{Introduction}

Galaxy clusters are impressive structures in optical images. In fact, when Charles Messier (Biviano, 2000) made a list of nebulae (nowadays known as galaxies) in 1784, he already noticed the clustering of objects in the Virgo constellation (nowadays known to host the Virgo cluster). Galaxy clusters span $1-2 h^{-1} \mathrm{Mpc}$ in radius, and turn up as agglomerations of elliptical-shaped galaxies with red colors. At a larger scale, galaxy clusters appear to be the junctions of the "cosmic web". The galaxy distribution in the universe resembles an enormous network: cosmic voids with few objects are separated by filamentary structures made of galaxies. Galaxy clusters, which are either scattered along the filaments or connect several of them, represent the densest regions in this gigantic web.

The formation of clusters (see a review in Voit, 2005) starts with perturbations in the primordial matter density field. These initial perturbations grow through dark matter dominated gravitational accretion and form gravitationally-bound structures. The structures continue to grow through matter infall or merging with other structures, although the rising importance of dark energy after $z \sim 1.0$ speeds up cosmic expansion and acts against the growth.

Galaxy clusters can be as massive as $10^{15} \mathrm{M}_{\odot}$. Most of the mass comes from the dark matter component. Galaxy clusters are the most massive gravitationally-bound structures in the universe, and compose the massive end of the dark matter halo mass function. The cluster mass distribution and its evolution history are sensitive probes of the $\Lambda \mathrm{CDM}$ cosmological model (other probes include Type Ia supernovae, baryon acoustic oscillations and weak gravitational lensing. See a review in Weinberg et al., 2013). Cluster abundance studies based on a variety of observational data sets have produced some of the strongest constraints on the dark matter and dark energy parameters (especially the $\sigma_{8}$ and $\Omega_{m}$ parameters, see a review in Allen et al., 2011).

Galaxy clusters are also important for astrophysical studies that investigate baryonic processes during cosmic structure formation. The enormous cluster mass forms a deep 
gravitational well, which traps most of the in-falling baryonic matter including gas and galaxies. In this high density environment, galaxies and the hot and cold gas experience frequent interactions, leaving rich observational traces. Astrophysical studies also enjoy the convenience that the mass and number density of clusters are relatively well understood from cosmological studies.

Advancement in wide field astronomical surveys has brought up great opportunities for both cluster cosmology and astrophysics studies. Galaxy clusters are rare objects in the universe. Depending on the redshift, the number density of clusters more massive than $10^{14} \mathrm{M}_{\odot}$ can be as low as $\sim 10^{-5}\left(h^{-1} \mathrm{Mpc}\right)^{-3}$ (Carlberg et al., 1997). However, the Sloan Digital Sky Survey that images $\sim 15,000 \mathrm{deg}^{2}$ of the sky has identified more than 25,000 $z<0.55$ clusters in this mass range (redMaPPer: Rykoff et al., 2014).

This dissertation includes a few studies I performed in the past five years. The work uses data from the Dark Energy Survey and the Sloan Digital Sky Survey to probe cluster astrophysics, or to be more specific, to probe the evolution of cluster galaxies. This introduction chapter introduces the general background for the studies. The specific topics are listed in Section 1.4. For the rest of this dissertation, Chapter 2 to 5 describe the studies with details, one chapter for each topic. Chapter 6 provides a recapitulation and an outlook discussion for the investigated topics.

It worths stressing that the topics investigated herein are also important for cluster cosmological studies. Probing cosmology models with galaxy clusters relies on finding these structures and approximating their masses. Cluster baryonic observables have been extensively utilized for these two purposes.

\subsection{Cluster Content}

Galaxy clusters contain about $90 \%$ of dark matter and $10 \%$ of baryonic matter (see Gonzalez et al., 2013, and the referenced papers). Many interesting phenomena about the formation of cluster baryonic content remain to be understood.

About $90 \%$ of the cluster baryons are distributed as the intra-cluster medium (ICM). This gas component gets heated to above $10^{6} \mathrm{~K}$ upon accretion into the clusters, producing $\mathrm{X}$-ray photons that render their hosts observable with X-ray telescopes (see a review in Sarazin, 1988). The heated gas may cool again towards cluster center as it releases energy with X-ray emissions, although the cooling is barely observed in a range of observations (see reviews for the phenomenon and the AGN feedback mechanism as an explanation in Fabian, 1994, 2012).

The other $10 \%$ of the cluster baryonic matter comes from the stellar content. Stars 
inside cluster galaxies make up 1\% of the cluster total mass (see Budzynski et al., 2014, and the referenced papers). Galaxy clusters constantly accrete galaxies or galaxy groups from the surrounding environment, especially from the cosmic filament environment. The merging of clusters also makes these structures grow bigger.

The cluster galaxy population has one prominent feature: most of them are no longer forming stars. Cluster galaxies generally appear to be red elliptical or S0 galaxies. These "red sequence" galaxies occupy a narrow region in the color-magnitude diagram (Bower et al., 1992a). The homogeneous existence of a "red and dead" galaxy population indicates that there exist mechanisms to quench star formation and transform galaxy morphologies, either before (pre-processing) or after (post-processing) cluster infall. Some of the key astrophysical processes include stripping (e.g., Bahé \& McCarthy, 2015) and strangulation (e.g., Peng et al., 2015).

Another prominent feature of the cluster galaxy population is the giant elliptical galaxy at cluster center. Dubbed the bright central galaxy or the brightest cluster galaxy (BCG), this object can be $\sim 10$ times more massive than other cluster members. The BCGs are the galaxy occupants of the bottom of the cluster potential well. The formation of these galaxies (De Lucia \& Blaizot, 2007) illustrates the hierarchical structure formation process predicted by dark matter and dark energy cosmology models. As clusters combine to form more massive ones, galaxies inside clusters also merge to grow in mass and size. This is especially true for BCGs.

It is likely that a big fraction of the cluster stellar content exists in the form of diffused stars not associated with cluster galaxies. This component consists of stars that got ejected into the intra-cluster space during galaxy interactions (see Contini et al., 2014, and the referenced papers). The stars are no longer gravitationally bound to the galaxies but still bound to the cluster. This component is known as the intra-cluster light (ICL). ICL tends to be concentrated around the BCGs, but still appears to be extremely faint (about $1 \%$ of the sky brightness). Measuring the properties of intra-cluster light requires deep imaging data that are exquisitely processed (e.g., Krick et al., 2006; Rudick, 2010). Limited by the observing and data processing requirement, the formation mechanisms and the properties of this component remain largely unknown. A few observations estimate that ICL forms up to $50 \%$ percent of the cluster stellar content (e.g., Zibetti et al., 2005; Krick \& Bernstein, 2007; Gonzalez et al., 2007; Toledo et al., 2011; Montes \& Trujillo, 2014; Giallongo et al., 2014; Presotto et al., 2014). 


\subsection{Multi-Wavelength Data}

Galaxy clusters leave imprints across a wide range of the electro-magnetic spectrum. The primary methods of discovering clusters utilize the emissions from their baryonic content.

For many clusters, the over-density of galaxies makes them easy to spot in optical images. The history of finding clusters in optical observations dates back to the 18th century (Biviano, 2000). The rising awareness about inhomogeneous galaxy distribution in the 1950s stimulated discoveries of clusters. Systematic cluster searches started (Abell, 1958) with visually inspecting photographic plates.

The use of charge-coupled device (CCD) in astronomy reduced technical barriers in obtaining precision photometry, which prompted the development of automatic cluster finding algorithms. Especially, precise measurements makes it possible to utilize the colors of red sequence galaxies (Gladders \& Yee, 2000) in cluster finding. Cluster redshift can also be inferred from optical colors (for example, see Bleem et al., 2015). Similar to the use of optical observations, galaxy clusters are discovered in infra-red images, although the data are more suitable for studying high redshift $(z>1.0)$ clusters because of the shifting of galaxy spectra (e.g., Muzzin et al., 2013).

In addition to identifying clusters, cluster masses can be inferred from optical imaging data. An economic mass estimator is the number count of cluster galaxies. (e.g., Rykoff et al., 2012). Another technique is weak lensing mass measurement which can directly probe cluster total masses (including the mass of dark matter) (e.g., Sheldon et al., 2004, 2009a; Johnston et al., 2007; Sheldon et al., 2009b).

As mentioned in the above section, galaxy clusters leave imprints in the X-ray wavelength range. The X-ray photons are primarily produced in the hot gas bremsstrahlung process. Cluster X-ray emissions can be as luminous as $10^{43} \mathrm{ergs} / \mathrm{s}$, and appear to be extended in images. The phenomenon was first observed with the M87 galaxy (Byram et al., 1966) in the Virgo cluster. Since then, clusters up to redshift $\sim 1.6$ (Santos et al., 2011; Tanaka et al., 2013) have been discovered. In addition, X-ray temperatures and luminosities have been utilized for accurately inferring cluster masses (see a review in Allen et al., 2011).

Comparing to the number of clusters discovered with optical (or infra-red) data (a few thousands), the sizes of X-ray cluster samples are modest (up to a few hundreds). Because the Earth's atmosphere is opaque to X-rays, X-ray telescopes need to mounted on artificial satellites in the outer space. Cluster X-ray discoveries are limited by the availability of observing resources. However, it is possible to discover thousands of clusters through Xray archival data searching. The $X M M$ cluster survey (Lloyd-Davies et al., 2011; Mehrtens 
et al., 2012; Viana et al., 2013) is a project that conducts this kind of searching.

Besides, galaxy clusters leave imprints on the cosmic microwave background (CMB) radiation. The energy of the CMB photons gets boosted when passing through the cluster hot gas, which is known as the Sunyaev-Zel'dovich effect (the SZ effect, Sunyaev \& Zeldovich, 1969, 1972). The Planck satellite (Planck Collaboration et al., 2014, 2015), the Atacama Cosmology Telescope (Marriage et al., 2011; Hasselfield et al., 2013) and the South Pole Telescope (Staniszewski et al., 2009; Reichardt et al., 2013; Bleem et al., 2015) surveys have provided cluster samples discovered with this effect. The SZ signals are known to be tightly correlated with cluster masses (see a review in Allen et al., 2011). Galaxy clusters are also expected to produce radio (see a review in Ferrari et al., 2008) and gamma ray emissions (Huber et al., 2013; Ackermann et al., 2014; The Fermi-LAT Collaboration \& Rephaeli, 2015; Ackermann et al., 2015), but the signals are less ideal for cluster discovery or cluster mass measurements.

\subsection{Wide Field Surveys}

Using uniform wide field data provides statistical power and reduces selection biases for cluster cosmology and astrophysics studies. This dissertation is primarily based on data from the Dark Energy Survey (DES). Since wide field DES data were not available at the time one of the studies (Chapter 5) was performed, we have made use of the Sloan Digital Sky Survey data for that work.

The Sloan Digital Sky Survey (SDSS) is a joint imaging and spectroscopic survey. It observes $\sim 15,000 \mathrm{deg}^{2}$ of the northern hemisphere sky in the optical wavelength range. Originally designed to measure Baryon Acoustic Oscillation, the SDSS has made many serendipitous contributions from discovering Solar system objects to studying distant quasars. The SDSS data have been used in more than 5,800 publications ${ }^{1}$.

The SDSS project uses a $2.5 \mathrm{~m}$ telescope located at the Apache Point Observatory. The imaging survey was conducted from 1998 to 2008. Images were taken under photometric conditions in multiple optical bands $(u, g, r, i, z)$ with a drift scanning technique. The image depth reaches $22.3 \mathrm{mag}$ in $i$ (point sources, 5 $\sigma$, York et al., 2000). For galaxy cluster science, the depth allows identifying clusters and studying their galaxy population to $z \sim 0.55$ (Hao et al., 2010; Rykoff et al., 2014).

The SDSS spectroscopic survey programs acquire spectra for many millions of targets selected from the imaging data. Different programs geared towards a variety of science goals have been conducted since 2000 (e.g., SEGUE for surveying galactic stars, BOSS for

\footnotetext{
${ }^{1}$ http://www.sdss.org/science/
} 
surveying luminous galaxies to $z=0.7$ ). This dissertation does not explicitly use SDSS spectroscopic data.

The Dark Energy Survey (DES) is the successor of SDSS in the category of extremely wide-field optical surveys. Dedicated to probing cosmology models with four different methods - type Ia supernovae, baryon acoustic oscillations, galaxy clusters, and gravitational lensing - DES is one of the most ambitious cosmology experiments currently in action. The program makes use of the newly-built, wide-field Dark Energy camera (DECam, Flaugher et al., 2015a) mounted on the $4 \mathrm{~m}$ Blanco telescope. DES is an imaging only survey, but spectroscopic fellow-ups are being performed with the Anglo-Australian telescope in collaboration with the OzDES project ${ }^{2}$.

The survey plans to image 5,000 $\mathrm{deg}^{2}$ of the southern hemisphere sky, with a few hundred $\mathrm{deg}^{2}$ overlapping (in the "Stripe 82" area) with SDSS. The area of the DES footprint is smaller, but the images will reach a significantly deeper depth. Because of a higher redshift goal, DES images are taken in five redder bands, $g, r, i, z$ and $Y$. A single exposure image reaches $23.6 \mathrm{mag}$ in $i$ and $22.9 \mathrm{mag}$ in $z$ (point sources, $5 \sigma$, Dark Energy Survey Collaboration et al., 2016). Full depth DES data consists of 10 exposures in each band. This will allow cluster discovery and cluster galaxy population studies to be performed to redshift 1.0.

DES started taking science quality images for science verification purposes in the fall of 2012 (see more details in Sánchez et al., 2014). The main survey have officially began since 2013 (Diehl et al., 2014) and will continue for 5 years.

\subsection{Dissertation Outline}

This dissertation focuses on characterizing the formation and evolution of cluster galaxies. More detailed introductions about the presented topics can be found at the beginning of the corresponding chapters.

Most of the work makes use of the DES science verification data. Since precision observational studies require precisely processed data, the dissertation starts with describing a new software package that helps improving cluster galaxy detection in DES data. The package has been published as a first-author paper (Zhang et al., 2015b) on the Publications of the Astronomical Society of the Pacific.

Two chapters are dedicated to the evolution of cluster galaxies (i.e., BCGs, Chapter 3) and cluster red sequence galaxies (Chapter 4). Because the evolution of ICL is closely related to the evolution of the galaxies, predictions about ICL evolution are drawn from the

\footnotetext{
${ }^{2}$ http://www.mso.anu.edu.au/ozdes/
} 
central galaxy study (Chapter 3). These two studies make use of DES science verification data and the cluster sample identified by the $X M M$ cluster survey. The cluster masses are derived from X-ray temperatures and luminosities. The cluster central galaxy analysis has been published on the the Astrophysical Journal as a first-author paper (Zhang et al., 2015c). The cluster red sequence galaxy study will be submitted soon.

Characterizing the cluster galaxy population also requires an understanding about the cosmic filaments which feed cluster galaxy accretion. Chapter 5 presents analyses on cosmic filament properties. The SDSS data were employed (Chapter 5) since DES had not started observing at the time when the analysis was undertaken. The work makes use of optically selected clusters by the gmBCG algorithm (Hao et al., 2010). The analysis is also published on the the Astrophysical Journal as a first-author paper (Zhang et al., 2013). 


\section{CHAPTER 2}

\section{Detecting Cluster Galaxies}

\subsection{Topic Introduction}

While deep optical images greatly facilitate the detection and measurement of clusters, they present some data processing challenges. There exist several software packages for processing wide-field optical images; each attempts to automatically detect astronomical sources and measure their properties (Jarvis \& Tyson, 1981; Beard et al., 1990; Maddox et al., 1990; Yee, 1991; Bertin \& Arnouts, 1996; Andreon et al., 2000; Lang et al., 2014, Lang et al., in prep). The SExtractor (Bertin \& Arnouts, 1996) package is among one of the most popular of these tools. These packages have greatly aided astronomical imaging studies, but may become inefficient when processing deep images crowded with objects. A common problem is the failure to detect objects blended with others, i.e., failing to deblend.

The Dark Energy Survey has adopted an advanced version of SExtractor in the data processing pipeline for catalog production (the Dark Energy Survey collaboration, in prep.). To handle crowded images, SExtractor has a deblending procedure that decides if a detected object should be further separated as several branching components. Upon application to DES data, a few SExtractor set-ups (including the DEBLEND_MINCONT, DEBLEND_NTHRESH,CLEAN_PARAM parameters and the image convolution filters) were explored, but the improved detection efficiency of blended sources comes at the cost of an increasing amount of spurious detections and deteriorated photometry measurements. Although an optimum deblending setting has been determined to maximize DES data quality for general purposes, some scientific studies involving crowded regions like galaxy clusters set a different, more demanding requirement.

The deblending problem also seems to plague other reduction packages. The data processing pipeline of the Sloan Digital Sky Survey (SDSS) is very different from that of DES (York et al., 2000; Lupton et al., 2001). However, there are also reports about suppressed completeness around bright objects (Adelman-McCarthy et al., 2006; Mandelbaum et al., 
2005), implying that deblending was also an issue. A few other sky survey programs tackle the deblending dilemma with two SExtractor runs, one optimized for detecting isolated objects and one optimized for detecting blended objects, but the output from the second run will need to be pruned and refined. This is usually achieved with precision profile fitting methods (GIM2D Simard et al. 2002; GALFIT Peng et al. 2002, 2010), which turns out to be too slow for extremely large data sets like those from DES. Another successful practice focuses on deblending images crowded with point sources, like the images of globular clusters (Federici et al., 1983; Stetson, 1987; Diolaiti et al., 2000; Savage \& Oliver, 2007), but the technique cannot be directly applied to extra-galactic imaging surveys. When deblending point sources, the intrinsic shape of every object can be more or less accurately estimated, but extra-galactic images are dense with galaxies, each with its own unknown shape, brightness, and size. Their images often overlap, making the identification of individual galaxies and measurements of their brightness a challenge.

In this chapter, we describe a new software package that aids blended source detection and photometry measurement - the Gradient And INterpolation based deblender (GAIN deblender). The GAIN deblender is a secondary package that operates on clean, already processed astronomical images, and requires one round of source extraction to be done before its application. It automatically identifies blended sources, separates them, and prepares the photometry measurement of each individual source. This software is primarily designed for extremely wide surveys like DES, and running speed is one of the biggest feature. The algorithms may also be of use for other data sets like those from HST and SDSS. We note that a future version of SExtractor featuring an improved deblender is under development (Bertin, private communication), which DES plans to implement upon its delivery. Meanwhile, our software provides a quick fix that assists current DES data production. It can also be combined to use with future SExtractor releases.

The rest of this chapter is organized as follows. We introduce the features and functions of the GAIN deblender in Section 2.2 and explain the algorithms in Section 2.3. We then analyze the effectiveness of this approach with real full-depth DES data, and the result is presented in Section 2.4. The software characteristics are summarized and discussed in Section 2.6.

\subsection{Software Features and Functions}

The Gradient And INterpolation based deblender (GAIN deblender) is written in c++ and IDL and can be acquired online ${ }^{1}$. It operates on processed images that have been cleaned:

\footnotetext{
${ }^{1}$ https://github.com/yyzhang/gain_deblend
} 


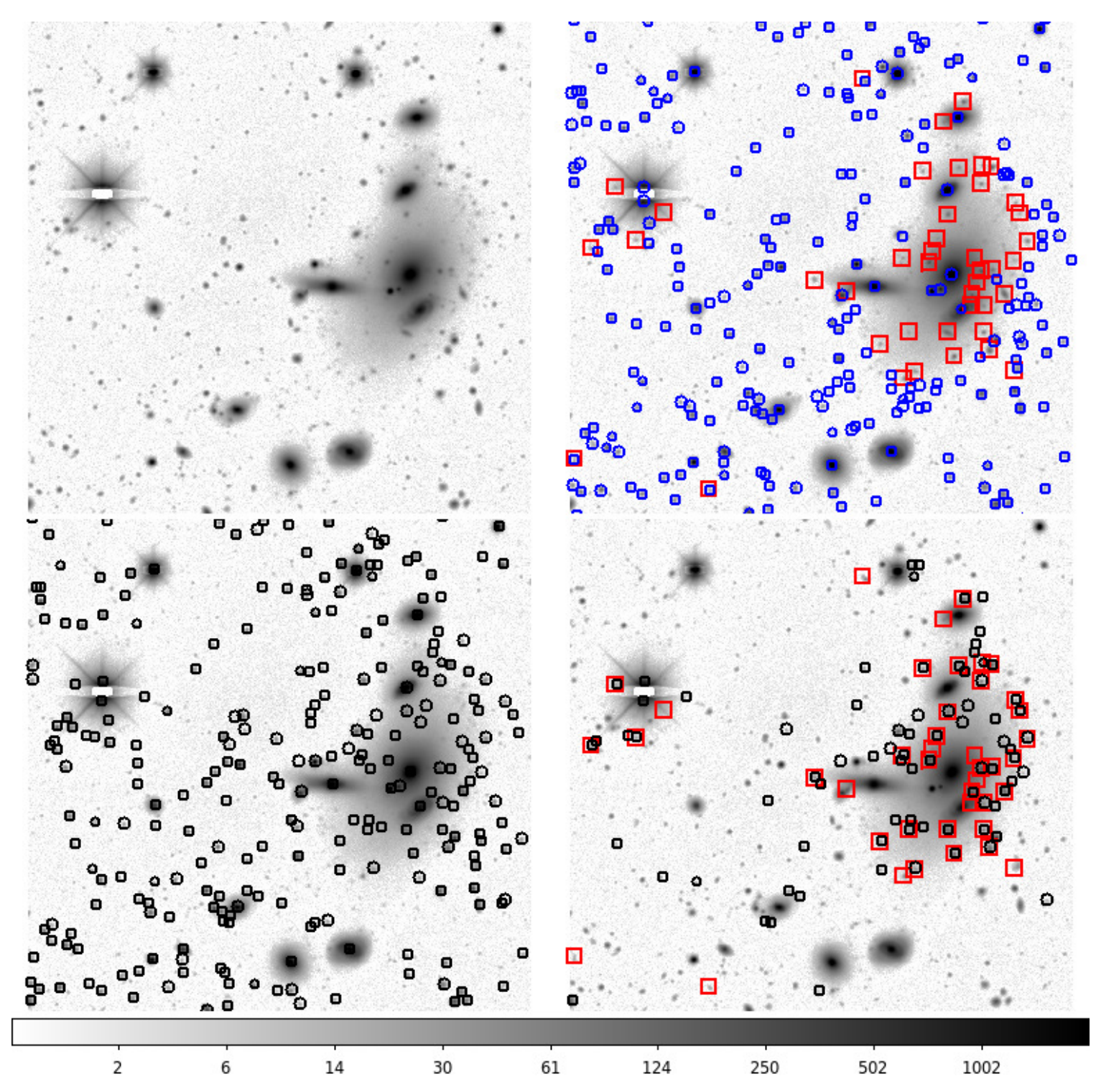

Figure 2.1: Upper Left: the linear combination of DES $r, i$ and $z$ coadd images for a cluster field. There is a bright star on the left and a brightest cluster galaxy on the right. Upper Right: same image with detected sources marked out. Blue circles are SExtractor detections (226 objects) using standard DES pipeline settings. Red squares (42 detections) are additional detections found through GAIN. Lower left: SExtractor detections with an aggressive deblending setting (DEBLEND_MINCONT $=1 \times 10^{-6}$ ), which tends to introduce many spurious detections. Lower Right: black circles (80 detections) are the additional Sextractor detections gained by more aggressive deblending. For comparison, GAIN detections from the upper right panel are shown again in this figure. In these figures, we only show sources brighter than 24.0 mag in $i$. 


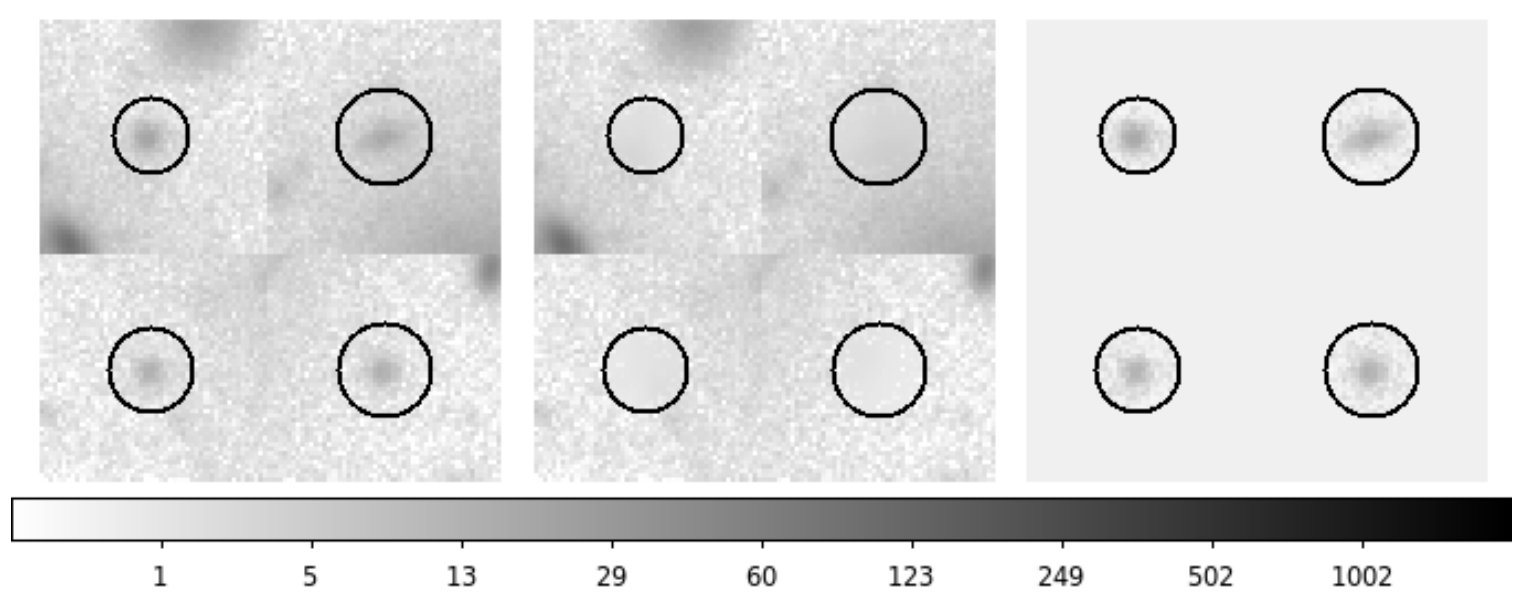

Figure 2.2: Left: $r$ band DES coadd images of four blended sources. The circles indicate the areas to be deblended. Middle: images that contain light from neighbors of the blended sources. Right: the residual between the left and middle panels, which shows the light from the blended sources within the circle.

flatfielded, background subtracted, with cosmic rays removed. We assume that the users have already done one round of source extraction by other means, and are using this package for finding blended sources that are missed in the previous procedure. The final output from GAIN are deblended (blending-effect removed) images of blended sources. GAIN does not include any catalog extraction module for producing catalogs from images. In our application in this chapter, we use SExtractor to produce source catalogs from the deblended images.

The first function of the software is the identification of blended sources. It begins by independently identifying all sources in the image, then matching its results to the user supplied object list. Sources not matched to the user supplied input list are kept as new sources to be extracted from the blended image. This procedure is illustrated in Figure 2.1. The upper left panel in Figure 2.1 shows a DES image containing a bright star and a brightest cluster galaxy. The upper right panel of Figure 2.1 shows sources detected by SExtractor (226 objects in blue circles) and some additional sources identified by GAIN (42 objects in red boxes). Note that this package is sensitive to image imperfections as is the case around the saturated star in the left half of the image. In general, the GAIN algorithm can find blended sources without introducing as many false detections as SExtractor with an aggressive deblending setting. This is illustrated by the 80 objects in black circles in the lower right panel of Figure 2.1.

The second function of the software aims to correctly assign the light in each pixel to the blended sources. This procedure is illustrated in Figure 2.2. Given a detected blended 
source, a region to be deblended is computed by our package. GAIN then interpolates for the light that comes from "background sources" as shown in the middle panel. The residual between the original image and the "background sources" interpolation is the light from the blended source alone (right panel). This constitutes the final output from GAIN. Users will need to employ an independent catalog-extraction software to construct catalogs from the deblended images.

We find GAIN to be useful for two scenarios. In many applications, GAIN would be used both to detect blended sources using the first component and to extract light for those sources using the second component. It may also be used by skipping the first step, and using the second component to extract light for user supplied blended sources. This step is recommended if the user has blended sources in their first round of source extraction and wants to obtain consistent photometry for all blended sources.

The GAIN package is fast enough to apply to wide field optical surveys. On a workstation computer equipped with Intel Xeon Processor E5645, running on a 10, $000 \times 10,000$ pixels image without parallelization, the source detection module takes approximately 300 seconds to identify 10,000 sources (without matching to a user supplied input catalog). The light separation module takes approximately 10 seconds for every 1,000 sources. The memory usage of this package depends on the size of the input image. It is generally more than twice the size of the image. For example, the total size of four tiles of DES coadd images is $\sim 3.5 \mathrm{~GB}$, the peak memory usage of GAIN running on these images can be higher than $8 \mathrm{~GB}$.

\subsection{Software Algorithms}

In the previous section, we mention that GAIN has two functions that can be combined for detecting blended sources and processing their images. In this section, we explain the algorithms used in these functions. The flowchart in Figure 2.3 demonstrates the computing steps during the application of the two functions.

\subsubsection{Source Detection}

The source detection component of GAIN aims to identify the local maxima in images which are associated with real objects. Our approach is inspired by the crowded field stellar photometry software DAOPHOT (Stetson, 1987). The presence of a separable astronomical object usually causes a local image intensity maximum. Unfortunately, many, even most, local intensity maxima are generated by image noise rather than real astronomical objects. 


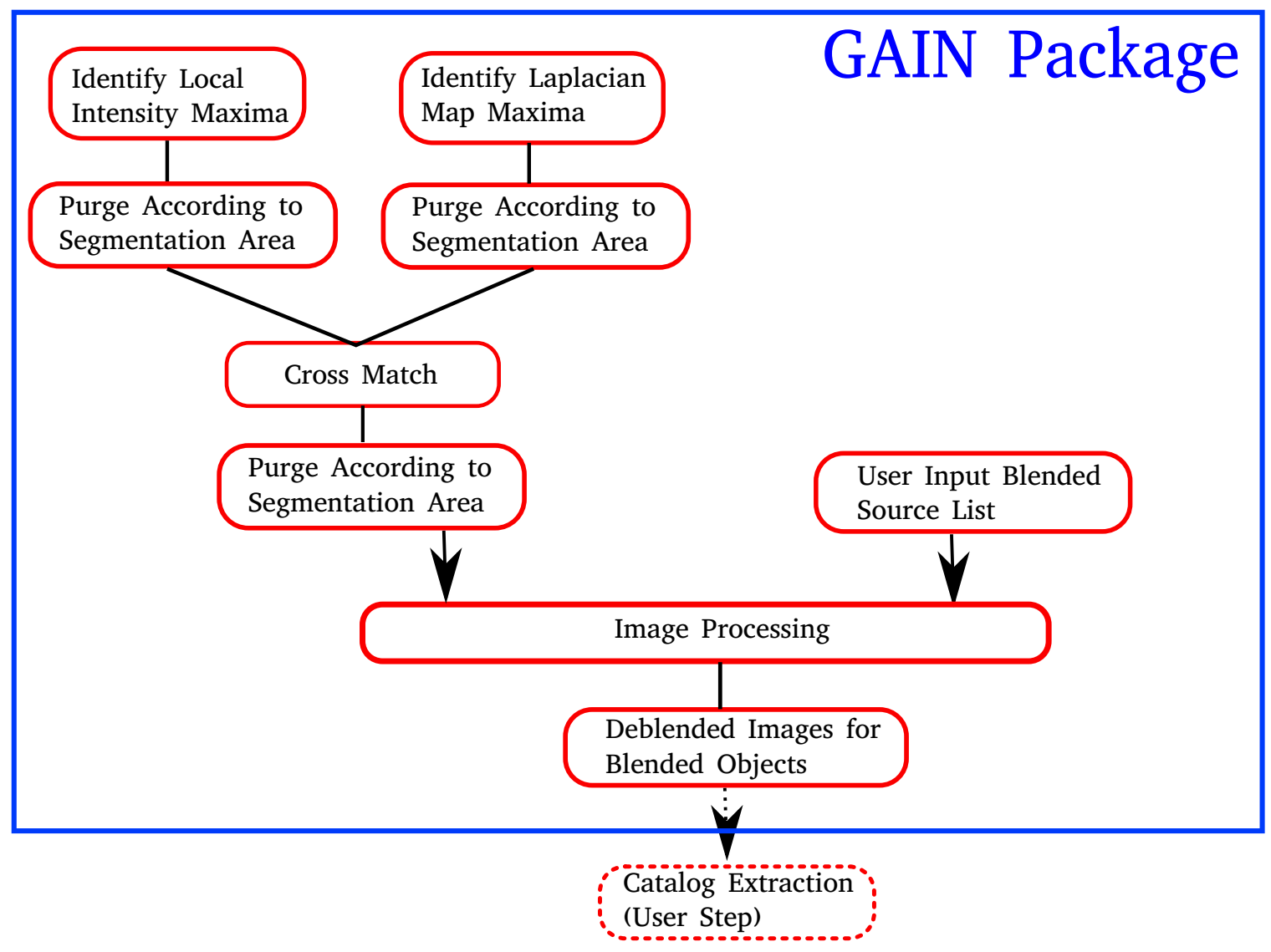

Figure 2.3: A flow chart demonstrating the GAIN computing steps explained in Section 2.3.

Procedures to eliminate these noise peaks are therefore necessary.

One approach to reducing the impact of noise is to smooth the whole image. While smoothing is very effective at eliminating noise, it also washes out the saddle points which separate close pairs of real astronomical sources, exacerbating the problem of blending. When close pairs are separated by distances around twice the FWHM of the seeing, and one source is brighter than the other, even very modest smoothing merges the two. To do the best job of deblending, we would like to avoid smoothing. Instead of finding maxima in smoothed images, GAIN uses the image segmentation procedure and the image Laplacian map to reduce the impact of noise.

Source detection in GAIN begins with identifying sources on raw, unsmoothed images, then purges the identifications assigned with low pixel area in the segmentation map. To further eliminate spurious detections, GAIN cross matches the remaining identifications to sources identified in a "weighted Laplacian" map (which we explain in Section 2.3.1.2). The software performs two other rounds of segmentation area purging during the "weighted Laplacian" step and during the "cross matching" step. 


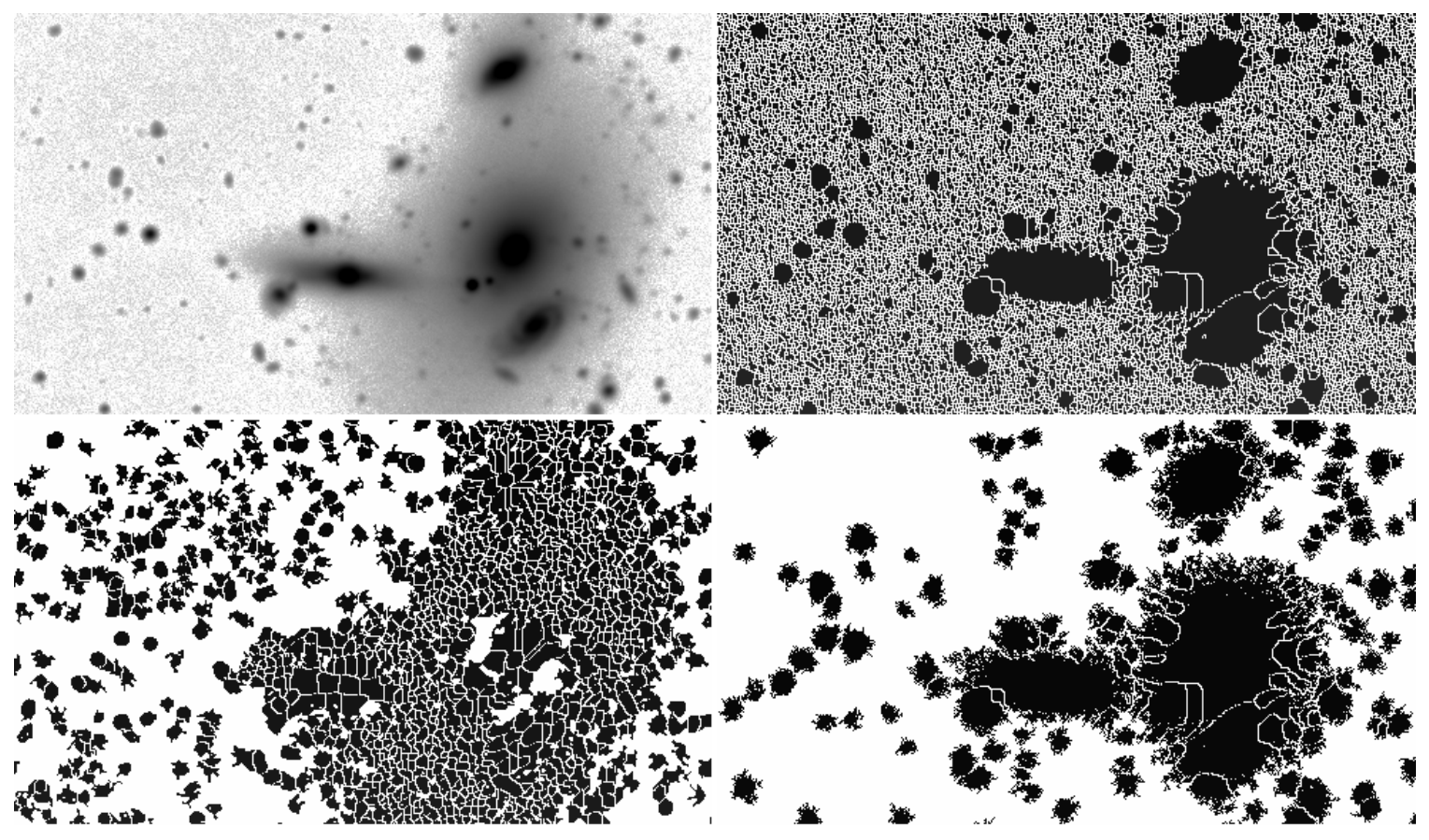

Figure 2.4: Upper left: Original DES coadd image, same as the one shown in Figure 2.1, but is zoomed in to best illustrate the segmentation procedure. Upper right: the segmentation map derived using all the local intensity maxima. Lower left: the segmentation map derived using all the local maxima of the weighted Laplacian map. Lower right: the segmentation map derived using the cross matched local maxima between the intensity map and the weighted Laplacian map. In the three segmentation maps, the empty white regions are the "boundarys" (see Section 2.3.1.1 for definition), and the pixels in black are associated with the seeds (local maxima from the intensity map, from the weighted laplacian map, and the cross matching between the two). 


\subsubsection{Segmentation of Images}

Purging of noise peaks can be aided by segmenting an image into separate regions, each associated with one element in the seed list of maxima. This seed list may include all local maxima, or may be produced by some other means, for example it could be a list of objects identified by SExtractor. In this first round of segmentation, we use image local intensity maxima, computationally defined as the pixels with intensity higher than the eight neighboring pixels. To segment the image, we use a simplified version of Meyer's watershed flooding algorithm (Meyer, 1992). To prime the segmentation procedure, we give each of the pixels in the seed list a unique region label. The goal of segmentation is then to label every pixel in the image as either belonging to one of the regions or residing in a boundary between the regions. The process begins by ranking all pixels in the image in descending order of intensity. As we move down the list, we apply the following procedure to each pixel.

1. If this pixel is already labeled (because it was in the seed list), its label remains unchanged.

2. If this pixel is unlabeled, and all of the neighboring pixels are unlabeled, this pixel is marked as a boundary. This should be true only for local maxima not found in the seed list.

3. If this pixel is unlabeled, and some or all of the neighboring pixels are already labeled, and their labels (except those labeled as boundary) are not all the same, this pixel is labeled as a boundary.

4. If this pixel is unlabeled, and some or all of the neighboring pixels are already labeled, and their labels (except those labeled as boundary) are all the same, this pixel is given that label. If all the labeled neighbors are labeled as boundary, this pixel will also be a boundary.

Note that this procedure differs from the original implementation of Meyer's algorithm. When the original seed list includes all of the local maxima (as shown in the upper right panel of Figure 2.4), the procedure above gives the same result as Meyer's, though it is much faster. When the seed list does not include all of the local maxima, as will be the case in our GAIN application, this algorithm yields thick boundaries (shown in the lower panels of Figure 2.4). This may not be desirable for computer vision applications like those for which Meyer's watershed algorithm was invented, but it is acceptable for our purposes. 


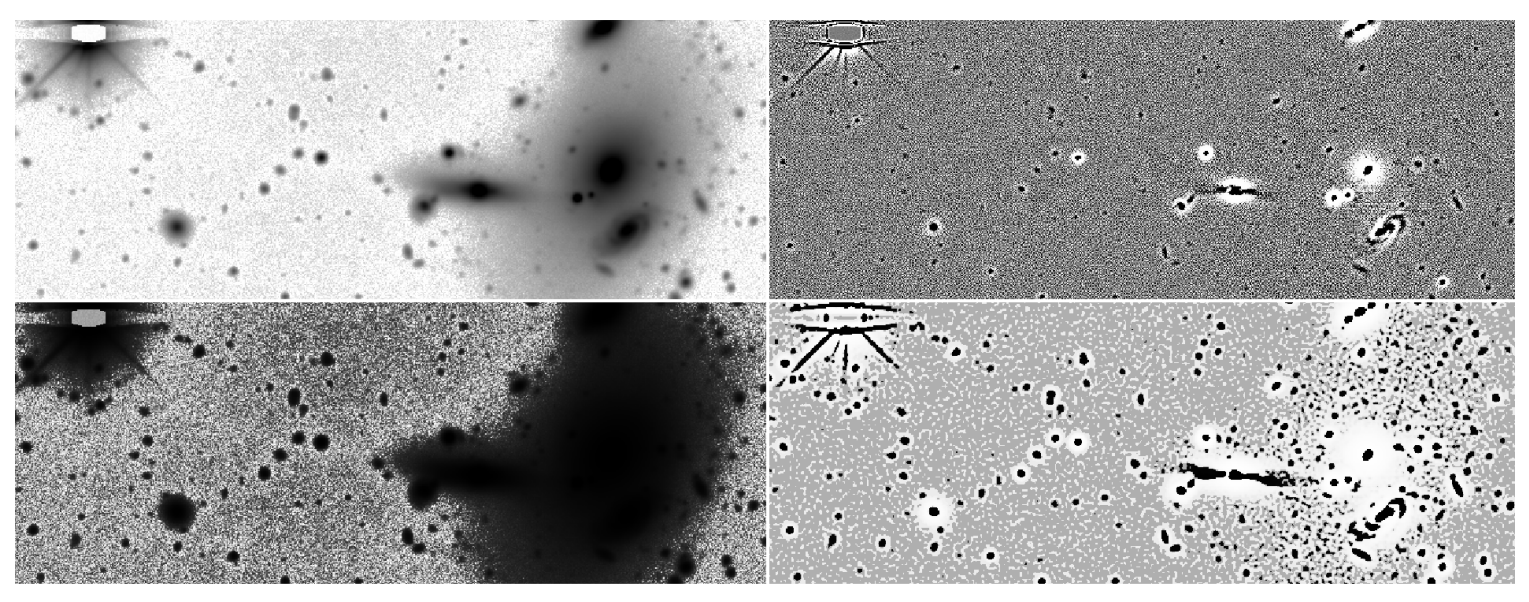

Figure 2.5: Upper Left: original DES coadd image, same as the one shown in Figure 2.1 and Figure 2.4. Upper Right: the Laplacian map of the same image. The fine features of the original image, like the spiral arms of the galaxy at the bottom right, are enhanced in the Laplacian map. Lower Left: intensity weighting map $\left(I_{w}\right)$ derived as described in Section 2.3.1.2. High intensity peaks are suppressed in this weighting map. Lower right: weighted Laplacian map in Section 2.3.1.2 which combines the Laplacian map with the intensity weighting map to bring out faint features.

After the segmentation is complete, we count the number of pixels labeled as belonging to each element of the original seed list. This number reflects the total area associated with each seed by the algorithm. We then purge false detections by eliminating seeds with segmentation areas smaller than an adjustable threshold value. For example, we purge all seed maxima associated with fewer than 27 pixels in DES coadd images. This threshold is set to reflect the the typical size of unresolved point sources in DES coadd images, but its value is finalized through trial and error, as are the other threshold values mentioned in this chapter.

\subsubsection{Cross Matching to Laplacian Maxima}

To populate an effective list of sources for deblending, GAIN generates a Laplacian map $(3 \times 3$ pixels Laplacian) of the original image. This is a useful approach because the contrast between real objects and their local background can be greatly enhanced in a Laplacian map. In terrestrial image processing applications, the Laplacian of Gaussian method is often used for edge detection (Lindeberg, 1993). In these applications, an image is first smoothed on some scale with a Gaussian kernel, and then the Laplacian of the resulting image is calculated. However, in astronomical images, real objects already have had their spatial extent smoothed by an instrumental PSF, so that finding features in the Laplacian is an effective approach to object detection. 
One limitation of this approach is that extended low contrast sources are not prominent in the Laplacian map. Since many of the sources indeed have low surface-brightness, we ameliorate this problem by weighting the Laplacian map with a transformed version of the original image intensity map. This weight map, denoted as $\mathrm{I}_{w}(x, y)$, is computed from the original image intensity map $\mathrm{I}(x, y)$ as,

$$
\begin{aligned}
& \mathrm{I}^{\prime}(x, y)=\mathrm{I}(x, y)-\min (\mathrm{I}) \\
& \mathrm{I}_{w}(x, y)=\left\{\begin{array}{r}
\mathrm{I}^{\prime}(x, y), \text { if } \mathrm{I}^{\prime}(x, y) \leq \operatorname{mean}\left(\mathrm{I}^{\prime}\right) \\
\log \frac{\mathrm{I}^{\prime}(x, y)}{\operatorname{mean}\left(\mathrm{I}^{\prime}\right)}+\text { mean }\left(\mathrm{I}^{\prime}\right), \text { otherwise }
\end{array}\right.
\end{aligned}
$$

We use the logarithmic values for high intensity pixels to suppress their weight. The weighted Laplacian map is then derived from the raw Laplacian map $\mathrm{L}(x, y)$ and the image weight $\operatorname{map} \mathrm{I}_{w}(x, y)$, as

$$
\mathrm{L}_{w}(x, y)=\mathrm{L}(x, y) \mathrm{I}_{w}(x, y)
$$

Unlike the original image intensity map, where pixel values range across many orders of magnitude, the pixel values of $\mathrm{I}_{w}(x, y)$ span a much narrower range. Finally, we smooth $\mathrm{L}_{w}(x, y)$ with a Gaussian function $(\sigma=1$ pixel for application to DES coadd images, value adjustable). Local maxima identified in this smoothed, weighted Laplacian image then become the seeds for the segmentation and purging step described in Section 2.3.1.1. For illustration, we show the smoothed, weighted Laplacian image of a DES coadd image in Figure 2.5. We also show the segmentation result using local maxima from this smoothed, weighted Laplacian image in Figure 2.4.

\subsubsection{Further Purging}

As a final step of cleaning, we cross match the complete list of local maxima from the original image with the list of local maxima from the smoothed and weighted Laplacian image. We retain those local maxima which have corresponding peaks (within 2 pixels or 0.53 ") in the smoothed and weighted Laplacian image. This list of matched local maxima then becomes the seed list for the segmentation process (illustrated in the lower right panel of Figure 2.4). Local maxima associated with sufficiently large areas in the segmentation map then form the final list of GAIN detected objects. 


\subsubsection{Matching to User Supplied Catalog}

The usual application of GAIN follows an initial round of source extraction using tools like SExtractor. GAIN aims to search for additional blended sources missed by these applications. For this reason, we match the GAIN source list to the user supplied source list, and single out those sources not identified by the original reduction as a list of newly identified, deblended sources.

For this matching procedure, the $(\mathrm{x}, \mathrm{y})$ coordinates of the user supplied sources are taken as input. Then in descending order of the intensity value at the user supplied sources' coordinates, we search for each source's nearest match in our source list from Section 2.3.1. All matches with a separation less than a threshold value (set to 10 pixels or 2.7" for our application to the DES data) are considered valid matches. The matched source is then removed from our list and the matching process continues. After removing all GAIN sources which match the user supplied list, the remaining sources constitute our list of newly identified, deblended sources.

\subsubsection{Deblend Blended Sources}

The most difficult aspect of blended object photometry is untangling the relative contributions of light from multiple sources. It is not uncommon for a faint source near a bright object to sit atop a background with photon count equal to - or in extreme cases several times higher than - the photon count of the source. To measure photometry for blended objects, we must account for the light contributed by their neighbors.

\subsubsection{Separating the Light}

To disentangle light from multiple sources, we use an image inpainting technique originally developed in the computer vision field. In this field, many techniques have been developed to recover damaged parts of an image, or to remove components that are unwanted. Our method is inspired by the Telea (2004) technique which is used to "inpaint" an image, i.e., to recover the texture of a small patch of an image from its surrounding pixels. It cannot re-create new patterns in the to-be-filled region, but rather fills them with a smooth background through interpolation. The problem this technique tries to solve is similar to our light separation problem. It allows us to estimate the light contribution from the more extended sources in the blended pixels. We choose the Telea (2004) technique over a variety of other available approaches (Bertalmio et al., 2000; Criminisi et al., 2004) because it is computationally efficient and has been extensively studied. 
The Telea (2004) technique works as follows. Given an intensity map, an unknown pixel can be inpainted with a value approximated from its known neighbors. Telea (2004) developed an efficient way to prioritize pixels in an unknown patch and determine the order in which they are inpainted, starting from the pixels nearest to the boundary and progressing inward (Sethian, 1996). This technique explicitly maintains a narrow band of pixels to be filled in as one of its features. Our implementation is adapted from the Telea (2004) technique, with a few modifications. A brief description follows.

1. Identify the regions to be inpainted: for each deblended source, this region is defined as a circle centered on the object, with an area equal to the segmentation area from Section 2.3.1.1. Pixels in this region are labeled as unknown, and the rest as known.

2. Initiate the narrow band: the narrow band is a list of pixels originally identified as unknown, that have at least one neighbor labeled as known. Pixels in the narrow band are prioritized in the ascending order of their original intensity value.

3. Begin inpainting: select the highest priority pixel from the narrow band and inpaint it. We explain how this is done in the next two items. After inpainting this pixel, label it as known, and check if it has any unknown neighbors. If there are any, add them to the narrow band list, re-prioritize the narrow band, and repeat this step until the narrow band is empty.

4. Inpaint a pixel: to inpaint one pixel, we fill it with the zeroth order approximation value from its known neighbors,

$$
\mathrm{I}(q)=\frac{\Sigma_{p} w_{p} \times \mathrm{I}(p)}{\Sigma_{p} w_{p}}
$$

with $w_{p}$ being the weighting for each neighboring pixel.

5. The computation of $w_{p}$ follows the original definition in Telea (2004) as

$$
w_{p}=\operatorname{dir}(p, q) \cdot \operatorname{dst}(p, q) \cdot \operatorname{lev}(p, q)
$$

with

$$
\begin{aligned}
& \operatorname{dir}(p, q)=\frac{(\vec{p}-\vec{q})}{\|\vec{p}-\vec{q}\|} \cdot \vec{N}(p), \vec{N}(p)=\nabla I(p), \\
& \operatorname{dst}(p, q)=\frac{1}{\|\vec{p}-\vec{q}\|^{2}} \text { and } \\
& \operatorname{lev}(p, q)=\frac{1}{1+\left|I_{\text {orginal }}(p)-I_{\text {orginal }}(q)\right|} .
\end{aligned}
$$


Here, $\vec{p}-\vec{q}$ is the vector from pixel $p$ to pixel $q$. $\operatorname{dir}(p, q)$ (dir stands for direction) evaluates if the $p$ and $q$ pixels are aligned with the image intensity gradient direction $\vec{N}(p)$. The gradient vector, $\vec{N}(p)=\nabla I(p)$, is approximated with the four neighboring pixels of $p$. $d s t(p, q)$ (dst stands for distance) evaluates the distance between $p$ and $q$. $\operatorname{lev}(p, q)$ (lev stands for level) evaluates the closeness of image intensity before the inpainting procedure.

At step 2, we prioritize the narrow band pixels according to their image intensity values. This deviates from the fundamental feature of the Telea (2004) algorithm in that Telea (2004) prioritize the narrow band in the order of pixels' distance to the original known and unknown region boundary, while the distance to the boundary is calculated from the fast marching solution to the Eikonal Equation (Sethian, 1996). This is designed to mimic the practice in manual inpainting that the pixels closest to the known region are filled first (Bertalmio et al., 2000, 2001). However, we prioritize pixels for inpainting using their intensity value rather than their distance to the known region. This is because pixels with lower intensity are less affected by the astronomical object we are removing, and filling them in first allows for more reliable background reconstruction. Also, we use Equation 2.3 for step 4 with zeroth order approximation rather than the first order approximation used in Telea (2004) because astronomical images are noisy, and the derivatives at pixel scale are unreliable.

After using the above method for "background" interpolation for one object, the interpolated image contains light from the object's neighbors. The difference between the original image and the interpolated image contains the extracted light for this deblended object.

\subsubsection{Catalog Production}

GAIN does not contain a module that produces source catalogs from deblended images. To produce a useful catalog of deblended sources, we need to measure magnitudes and shapes, as well as to classify each as a star or a galaxy. Many packages capable of doing this are available (Bertin \& Arnouts, 1996; Simard et al., 2002; Peng et al., 2002, 2010a), and a user might choose their favorite. For our application to the DES data, we use SExtractor (Bertin \& Arnouts, 1996). The tests described in the following sections are also based on the application of SExtractor. We provide the wrapper code for such an application in our package. Applications of other software, like GALFIT(Peng et al., 2002, 2010a), are possible as well.

When choosing software for cataloging, we advise users to consider a few things: 

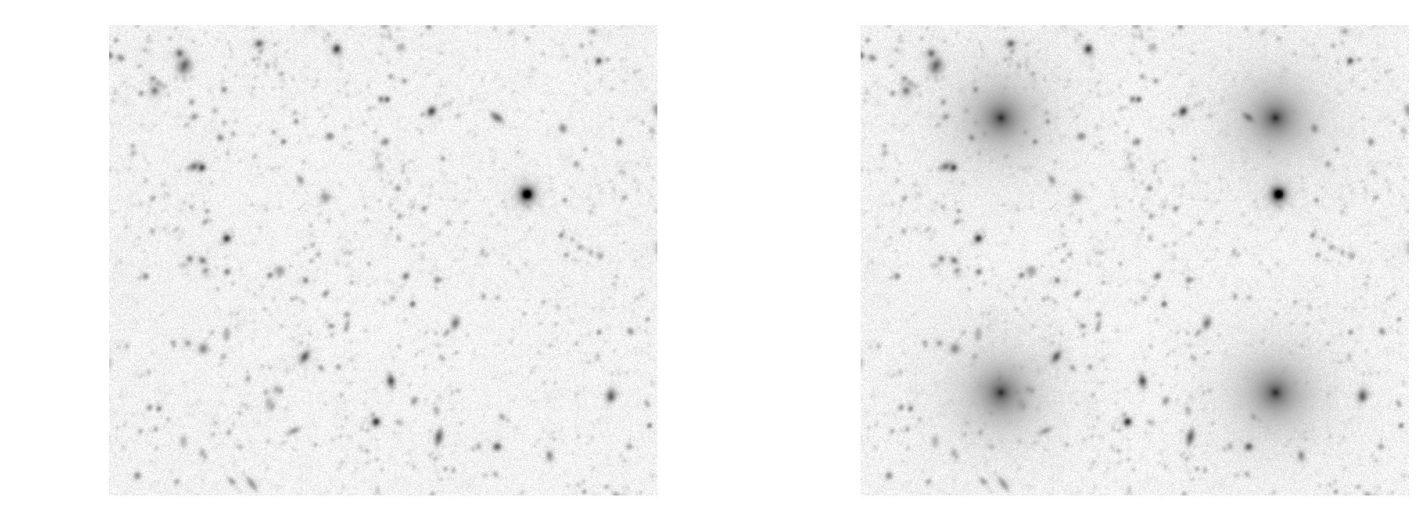

Figure 2.6: Left: An $r$ band DES coadd image. The 10 sigma limiting magnitude of this image is $25.3 \mathrm{mag}$ as measured from the SExtractor mag_auto uncertainty. Right: The same image after adding simulated BCGs. The size of these two images is approximately $3.15^{\prime} \times 3.15^{\prime}$. The apparent magnitude of the simulated BCGs is approximately $\sim 19$ mag.

1. The light extracted image of one object may be smaller than the area that contains all of its light. One should consider how to reconstruct/account for the light of the object outside this region.

2. Because of the above constraint, photometry from model fitting is probably more appropriate. With our application of SExtractor, we find that Kron (Kron, 1980) and Petrosian (Petrosian, 1976) magnitudes can provide reliable photometry.

3. Some star/galaxy separation methods may not work on light extracted images. When we use SExtractor on such images, we find that the class_star quantity fails most of the time because of the small area used for light separation. Star/galaxy separation using SExtractor spread_model quantity (a classifier that evaluates object profile with local PSF, Bertin, 2011) is still effective.

\subsection{Methods Validation}

We verify the performance of the following aspects of GAIN : photometry measurement, source detection completeness, and source detection purity. We also include a modest test on star/galaxy separation as part of the photometry test.

We want to test this package on optical images with complex deblending challenges, while all the sources in these images are known and already reliably measured. Our principal goal is to improve deblending around bright cluster galaxies, so we designed a test 
to simulate this challenge by adding simulated Brightest Cluster Galaxies (BCGs) to real deep optical images. For this test, we use deep coadd images from DES. Cleaning of individual exposures, coaddition of the images, and initial extraction of sources are all done using standard data processing pipelines from the DES collaboration (Mohr et al., 2012). We then select regions with few bright stars or real BCGs, so that deblending is not an important issue before the addition of a simulated BCG. Object catalogs extracted from these images are then used as "truth tables" in our testing procedure. When we add simulated BCGs to these images, some objects which are initially isolated and clean become blended, giving us a well understood deblending challenge to test against.

In Figure 2.6, we show an image before and after adding simulated BCGs. For the results presented in this section, we make simulated galaxy images of Sersic profile with Sersic index $n=4$ and Sersic radius $\operatorname{Re}=10^{\prime \prime}$ at $\sim 19.0$ magnitude (exact values vary depending on how brightness is measured). We convolve these profiles with the PSF function and add them into the image. We also ran the test with simulated galaxies of different magnitudes ( \pm 2 magnitude) and different Sersic parameters. The results are qualitatively independent of these changes. In this test, we combine GAIN with SExtractor for photometry measurement. We compare the performance of this set-up to the result from solely using SExtractor. Throughout the test, the major SExtractor deblending parameter, DEBLEND_MINCONT is set at 0.001, which is found to be optimum for processing Dark Energy Survey early data.

We note that the algorithm we describe in this chapter is designed for deblending between a satellite object and its much brighter neighbors. It may also be desirable to deblend closely spaced pairs and triples of astronomical objects. GAIN can indeed help with this kind of deblending problem, but its performance in these applications remains unverified. Because pairs or triplets do not always cause local maxima, it is hard to distinguish them from extended sources without using the image PSF (which is being implemented in a future version of SExtractor). GAIN is not optimized for this kind of deblending.

Finally, to thoroughly evaluate the deblending problem, the performance of GAIN, and more importantly the detection and photometry measurement of cluster galaxies in DES, one may wish to compare a data set with much higher resolution to DES data. A project comparing HST and DES data to study the DES data processing performance and its scientific effects is in progress (Palmese et al., in prep.). 
(a)

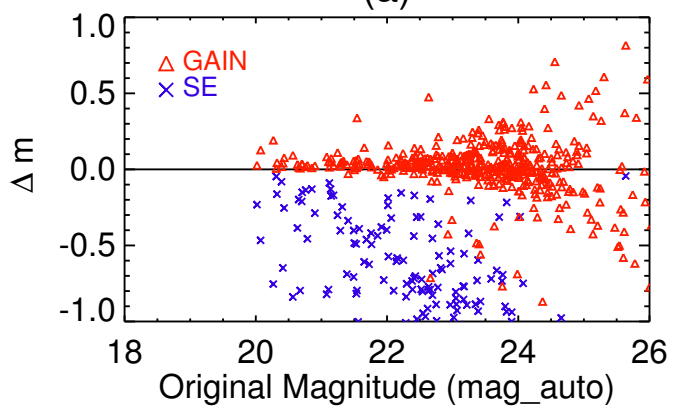

(c)

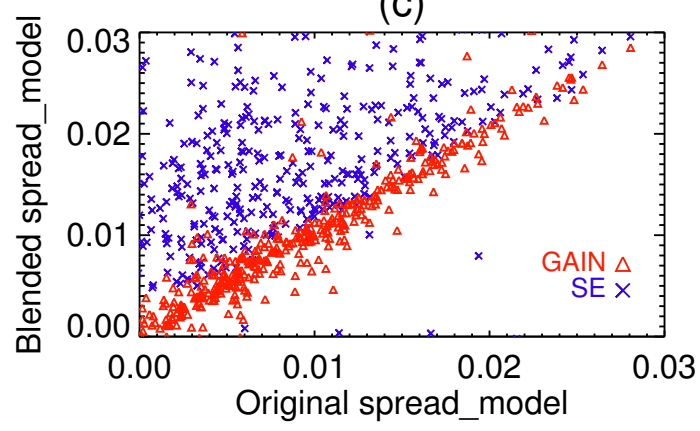

(b)

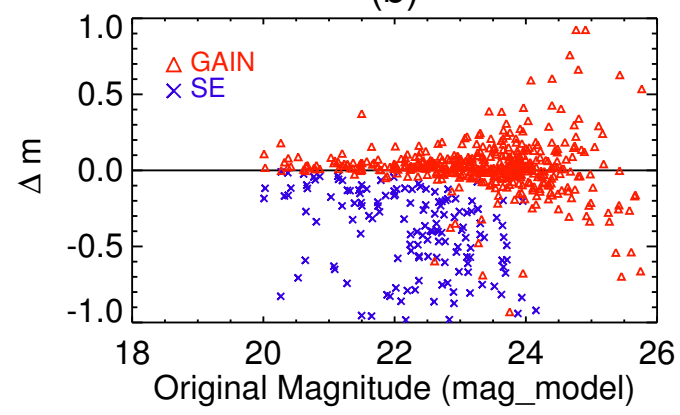

(d)

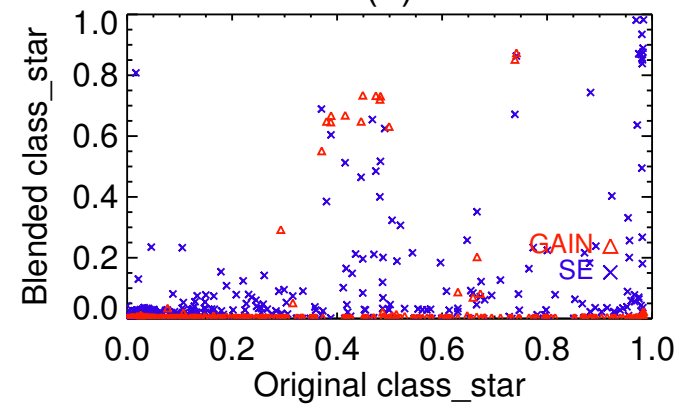

Figure 2.7: Comparison of photometry measurements and star/galaxy separation quantities with SExtractor using the "global background" setting. (a)(b) Offsets between the "truth" magnitudes and measurements from altered images for "artificially" blended objects. (c)(d) Comparison of star/galaxy separation quantities for "artificially" blended objects. 
(a)

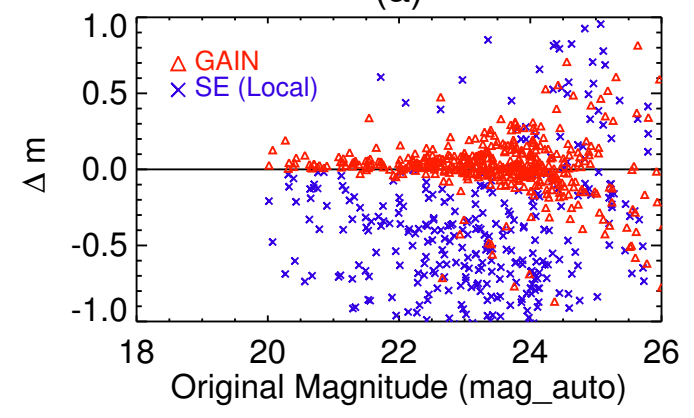

(c)

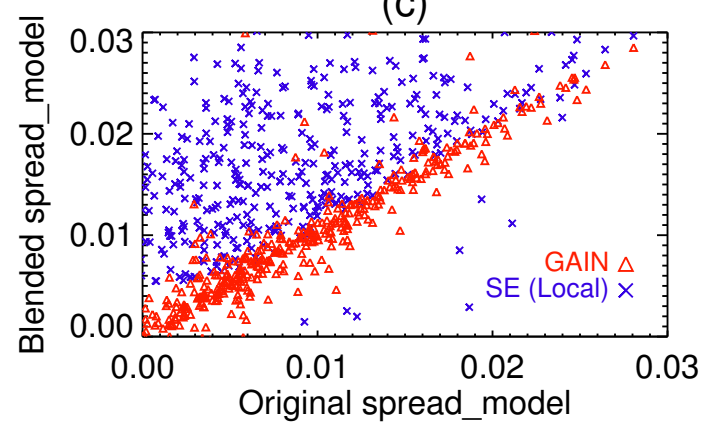

(b)

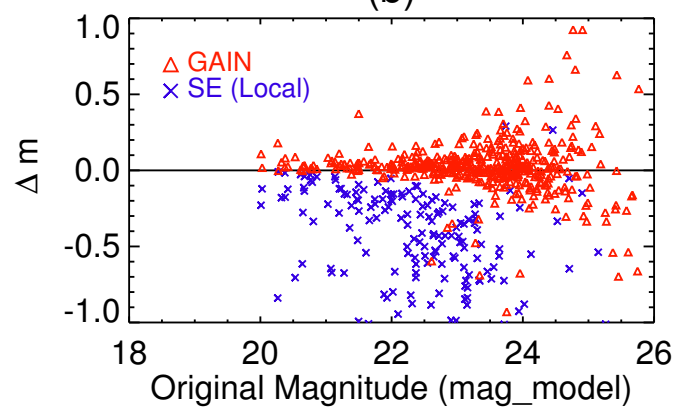

(d)

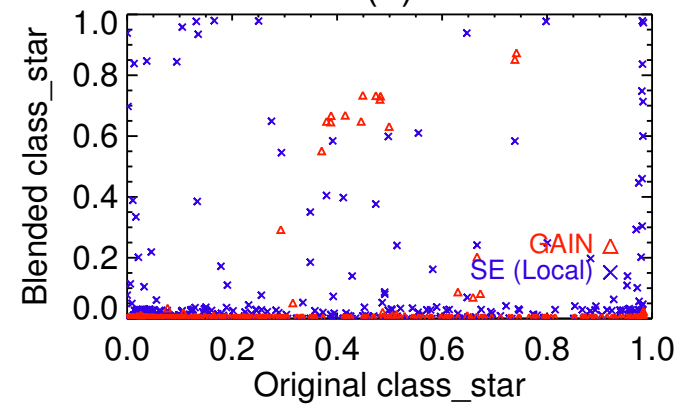

Figure 2.8: Comparison of photometry measurements and star/galaxy separation quantities with SExtractor using the "local background" setting. (a)(b) Offsets between "truth" magnitudes and measurements from altered images for "artificially" blended objects. (c)(d) Comparison of star/galaxy separation quantities for "artificially" blended objects. 


\subsubsection{Photometry Measurement}

To test whether GAIN can improve photometry measurement for blended sources, we use it to measure sources that become blended in the altered coadd image. We run SExtractor on the unaltered image as well as the image with simulated BCGs. We select sources that are flagged to be unblended (SExtractor flag $=0$ ) and isolated in the original image but become blended (SExtractor flag = 3) upon the addition of simulated BCGs. As these objects are considered to be clean in the original image, we treat their photometry measurement from this image to be the truth. In the images altered with simulated BCGs, we compare measurements of these sources from SExtractor directly and from SExtractor with GAIN implementation to their "truth" values. The result is shown in Figure 2.7 (a)(b).

Because light from simulated BCGs in the altered image is not completely accounted for in the basic SExtractor reductions, the blended sources typically have their brightness overestimated, often by as much as $0.5 \mathrm{mag}$. Comparing to model magnitudes (Bertin, 2011; Desai et al., 2012) in the truth table, Kron magnitudes (mag_auto) from the blended image are subject to more bias than model magnitudes. When GAIN is implemented, the measurements are significantly improved: both model magnitudes and Kron magnitudes for these "artificially" blended sources appear to be unbiased.

The photometry measurements from SExtractor in Figure 2.7 are obtained with the "global background" evaluation setting. In Figure 2.8, we show comparisons adopting SExtractor local background setting. A local background setting does help diminishing the biases, but is not sufficient to eliminate them. In addition, the scatter of photometry measurement is much larger with a local background setting.

In addition to magnitude measurements, we also include a modest comparison of star/galaxy separation parameters for these sources. This is shown in Figure 2.7 (c)(d) and Figure 2.8 (c)(d). We find that the class_star quantity has become ineffective for star/galaxy separation as discussed in Section 2.3.2.2. Another star/galaxy separation parameter spread_model appears to remain effective.

\subsubsection{Purity and Completeness}

In this section, we examine the improvement of completeness (did we recover all real objects) and purity (are all the new deblended objects real) after implementing GAIN.

After introducing simulated BCGs into deep optical images, issues associated with the deblending procedures emerge: object detection becomes incomplete and spurious detections appear. We use GAIN to improve deblending and then examine its impact on detection completeness and purity. Note that the face values of completeness and purity presented 


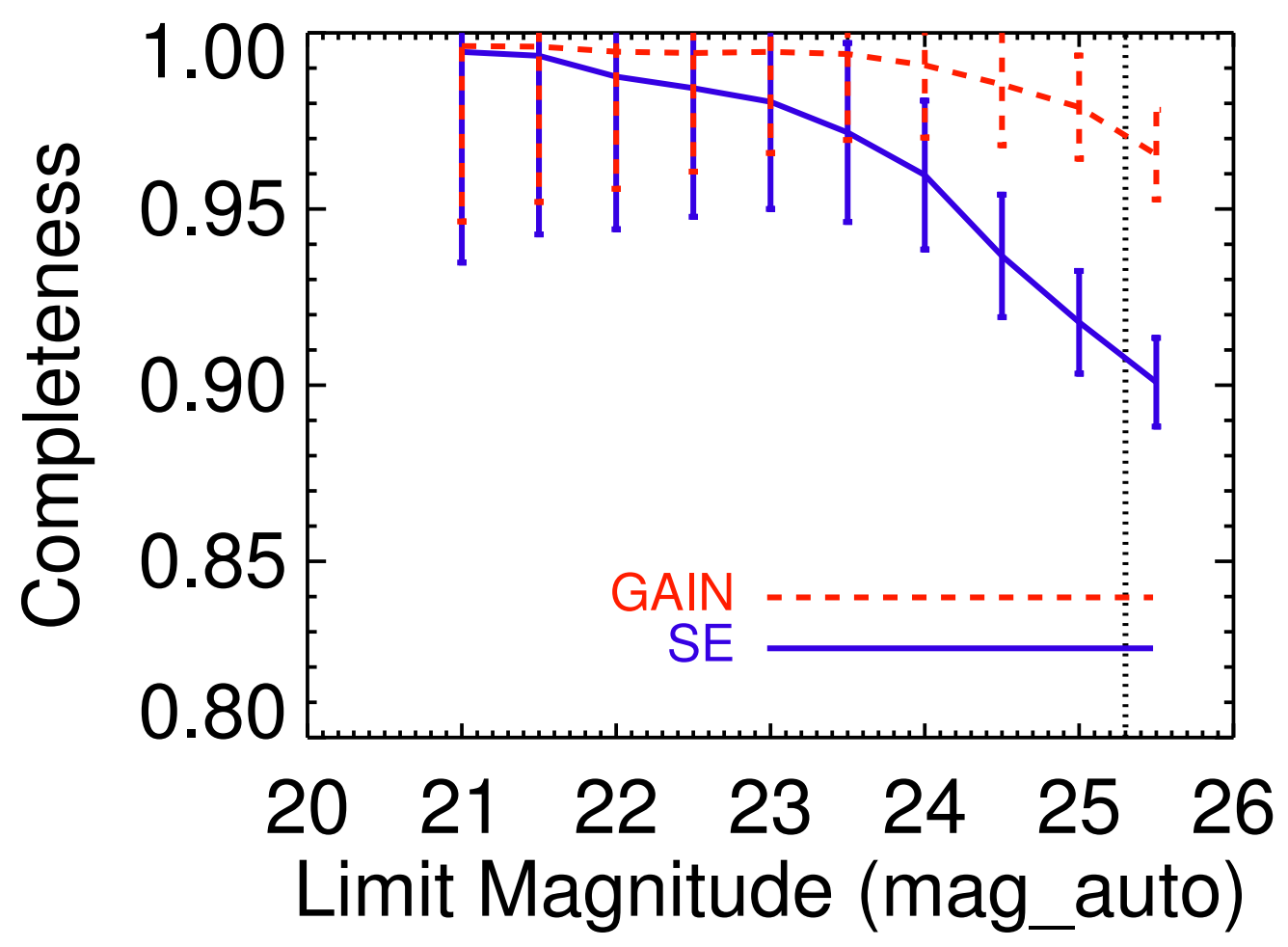

Figure 2.9: Completeness of the $\mathrm{C} 1$ catalog (blue solid line, from SExtractor) and completeness of the combination (red dashed line) of $\mathrm{C} 1$ and $\mathrm{C} 2$ (C2 from GAIN) as computed in Section 2.4.2.1. The non-negligible incompleteness of SExtractor catalog can be improved by GAIN all the way to 25.5 mag. The vertical dotted line shows the 10 sigma limiting magnitude of the image that the test is performed on. The errors in this plot are estimated assuming poisson distribution. 


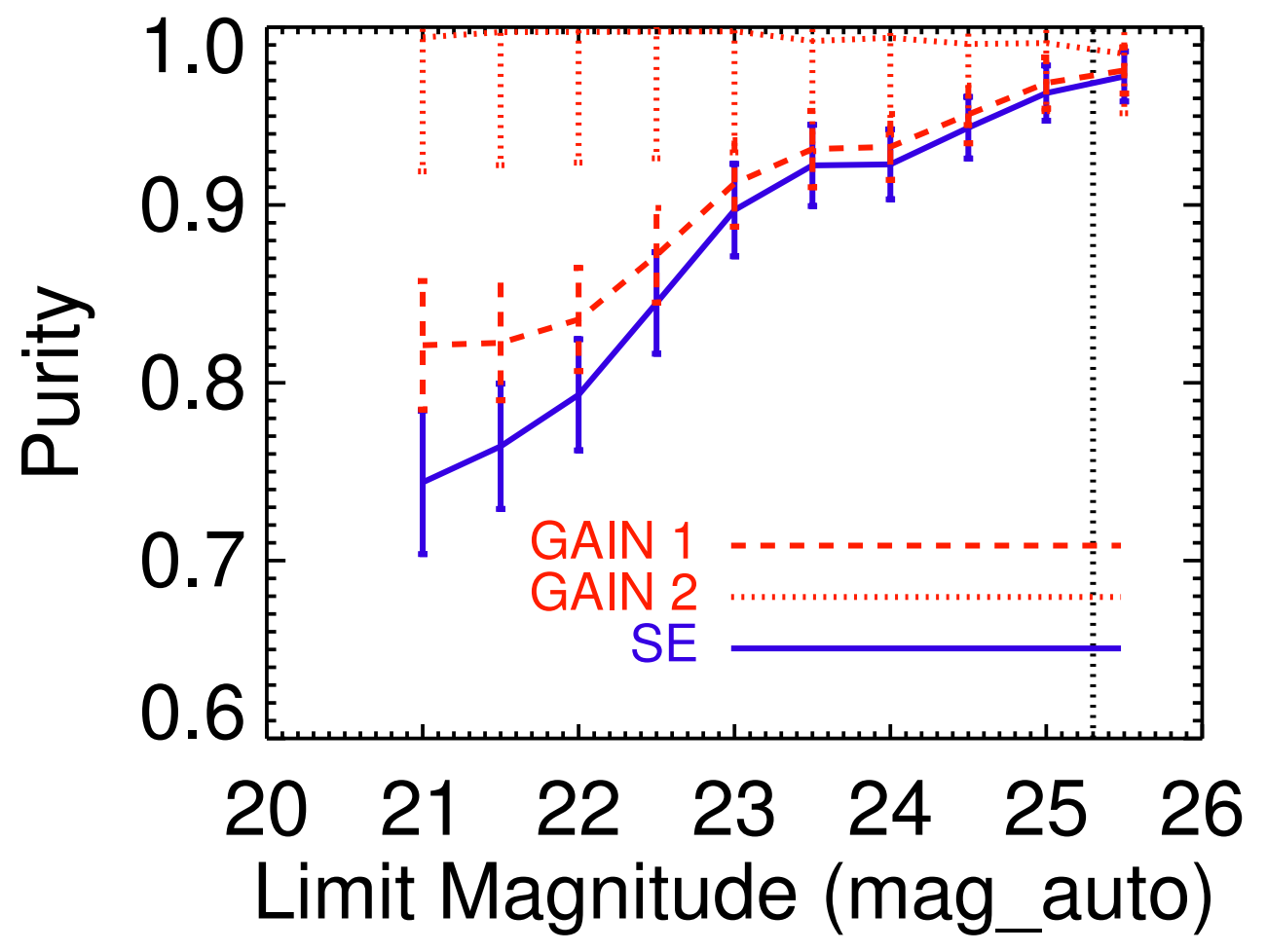

Figure 2.10: Purity of C1 (blue Solid line) by SExtractor and C2 (red dotted line) by GAIN and purity of the combination of $\mathrm{C} 1$ and $\mathrm{C} 2$ (red dashed line) as computed in Section 2.4.2.2. The unsatisfying purity of $\mathrm{C} 1$ at the bright end indicates that the deblending procedure of SExtractor is prone to introduce spurious detections. Also, the purity of the combination of $\mathrm{C} 1$ and $\mathrm{C} 2$ is affected by the purity of $\mathrm{C} 1$. On the other hand, the sources contained in C2 (GAIN output) are highly consistent with the previous running. The vertical dotted line marks the 10 sigma limiting magnitude of the image that the test is performed on. The errors in this plot are estimated assuming poisson distribution. 
in this section should not be taken as estimations for real astronomical images, as we are imposing exaggerated deblending difficulty in the testing images. The test here are only meant to show the effectiveness of GAIN with extreme situations.

\subsubsection{Completeness}

For the completeness test, we first run SExtractor on the unaltered coadd image, and use the resulting catalog as the Truth Table 1 (TT1). Running GAIN on the original, unaltered coadd image produces a catalog of blended sources not detected in Truth Table 1, and we use this list of additional sources as Truth Table 2 (TT2). The combination of TT1 and TT2 is then used as the total truth table (TT) in our completeness and purity tests. We then insert simulated BCGs, run SExtractor on the image to produce Catalog $1(\mathrm{C} 1)$, and run the GAIN package to search for blended sources and extract a supplemental Catalog 2 (C2). We then take all objects brighter than some magnitude limit from TT and match them to the $\mathrm{C} 1$ and $\mathrm{C} 2$ catalogs.

The matching is done in descending order of brightness for objects in the Truth Tables. For one object in the TTs, we search for the object that is nearest in $\mathrm{C} 1$ or C2. If the nearest neighbor from $\mathrm{C} 1$ or $\mathrm{C} 2$ is separated less than 5 pixels to the TT object, we claim this object as matched. Once a $\mathrm{C} 1 / \mathrm{C} 2$ object is used as a match, it is removed from the list available for matching. The TT sample is matched to the $\mathrm{C} 1$ or $\mathrm{C} 2$ sample deeper by 1 mag to ensure that the completeness evaluation is not subject to photometry measurement scatters. We evaluate completeness by computing the ratio between the number of matched objects and the total number of objects in the TT sample. We compute this quantity for SExtractor by matching TT1 to $\mathrm{C} 1$ and for GAIN improved catalogs by matching TT to the combination of $\mathrm{C} 1$ and $\mathrm{C} 2$. The result is shown in Figure 2.9.

After the image is altered by simulated BCGs, a small but non-negligible fraction of sources are missed from SExtractor data reduction, especially at the faint end. The situation is noticeably improved after the application of GAIN, demonstrating the effectiveness of the software.

\subsubsection{Purity upon Deblending}

As there is no clear definition of "real" objects in the DES images, for the purity test, we focus on evaluating the number of spurious detections introduced by the deblending procedure rather than categorizing objects as "real" or not.

To test the purity of the catalogs, we match $\mathrm{C} 1$ or $\mathrm{C} 2$ from the altered coadd images to Truth Tables from un-altered coadd images. The procedure is similar to the completeness 
test in Section 2.4.2.1, except that we match Catalogs to the Truth Tables instead of match Truth Tables to Catalogs. We match the $\mathrm{C} 1$ or $\mathrm{C} 2$ sample above a magnitude limit to the $\mathrm{TT}$ sample deeper by 1 mag. We match $\mathrm{C} 1$ to $\mathrm{T} 1, \mathrm{C} 2$ to the combination of $\mathrm{T} 1$ and $\mathrm{T} 2$ and also the combination of $\mathrm{C} 1$ and $\mathrm{C} 2$ to the combination of $\mathrm{T} 1$ and $\mathrm{T} 2$. We calculate purity as the ratio between the number of matched objects in $\mathrm{C} 1$ or $\mathrm{C} 2$ sample and the total number of objects in the sample. The result is shown in Figure 2.10. In this plot, GAIN 1 is the purity for the combination of $\mathrm{C} 1$ and $\mathrm{C} 2$, while GAIN2 is the purity of $\mathrm{C} 2$ alone. The purity of SExtractor (SE) is evaluated as the purity of $\mathrm{C} 1$ alone.

In Figure 2.10, purity of C1 (SE) lowers toward the bright end, and is less than $80 \%$ at magnitude 21 . This is partially caused by deteriorated photometry and astrometry measurements of blended objects. Spurious and real objects in $\mathrm{C} 1$ are biased brighter because of blending (see discussion in 2.4.1), which affects the bright end of the purity test. The purity of the combination of $\mathrm{C} 1$ and $\mathrm{C} 2$ (GAIN 1) is also negatively influenced by spurious detections in $\mathrm{C} 1$. For $\mathrm{C} 2$ (GAIN 2) alone, the sources contained in $\mathrm{C} 2$ are consistent with sources contained in $\mathrm{T} 1$ and $\mathrm{T} 2$ to $\sim 99 \%$. Figure 2.10 indicates that while performing the deblending procedure, SExtractor is likely to introduce spurious detections but GAIN is not.

\subsection{Discussion}

In this work, we describe modest tests showing that GAIN improves the reliability of photometry for blended objects. However, these tests are only designed to demonstrate the effectiveness of GAIN, and we recommend caution before applying these test results to new analyses. The completeness, purity, and photometry test results presented here should be considered valid only for this application of SExtractor and GAIN. GAIN is a supplement to object finding and photometry packages like SExtractor, and its performance inevitably depends on details of its image processing partner. In this work we compare the SExtractor and GAIN combined photometry output to SExtractor output, but do not investigate the original SExtractor photometry measurements.

A key element in any discussion of galaxy photometry is the evaluation of background light, which mostly comes from the sky. No photometry measurement algorithm will produce reasonable results if the sky background is inaccurately estimated. For this reason, any tests of photometric reduction algorithms must address background subtraction. GAIN does not perform photometry measurements or sky subtraction, so its connection to this issue is remote. Nevertheless, we have tested GAIN's susceptibility to imperfect sky background subtraction by performing an additional round of background subtraction for the 
test images used in Section 2.4. We employ the SExtractor local background evaluation function for this procedure, and then apply GAIN to these images that have went through an unnecessary (the images have already been background subtracted) and wrong (using improper SExtractor background evaluation setting) round of background subtraction. The results of GAIN application in this scenario remain quantitatively similar to those reported above, confirming our expectation that background subtraction has little impact on GAIN efficacy.

While the background estimation in this test is improper, it is not outrageously wrong. However, if it had been outrageously wrong, no photometry algorithm would produce reasonable results. This test at least shows that GAIN is not sensitive to imperfect background subtraction. Note that when performing image interpolation for blended sources, GAIN does perform an additional round of local background subtraction, in which the "background" light is contributed by neighboring objects. Our test in Section 2.4 have already verified GAIN's effectiveness on this aspect.

When applying GAIN for a specific science analysis, one may wish to design additional tests that focus on aspects of GAIN relevant to the analysis. For this work, aimed at deblending galaxies in crowded cluster cores, the real image plus simulated galaxy approach outlined in Section 4 seems to be the most relevant approach. We also considered tests using simulated images, but found them more difficult to interpret. Completeness and purity measures vary with environment, and objectively distinguishing different environments is not trivial. Finally evaluating the realism of the simulated images without a specific scientific goal in mind is challenging. It is for these reasons that we have chosen to test GAIN with this real image plus simulated galaxy approach.

Finally, GAIN is not a substitute for packages that would yield precision photometry measurement for blended objects (GALAPAGOS Barden et al. 2012; Galametz et al. 2013; GASPHOT D'Onofrio et al. 2014). These packages tend to perform two rounds of SExtractor source extraction with the second round tuned to pick up faint blended sources. As the second round of source extraction tends to yield many spurious objects and biased photometry, precision photometry fitting software needs to be employed to purge and refine the final catalog. Compared to these packages, the biggest advantage of GAIN is speed. The multipass approaches used on crowded fields are generally too slow for full wide field survey data, limited by the speed of precision photometry fitting. For example, the GASPHOT package is about 100 times slower than GAIN. It took GAIN 7 days to run for $300 \mathrm{deg}^{2}$ DES science verification data on a small computer cluster, but it would take GASPHOT 1.92 years for the same field. GAIN is strikingly efficient in terms of computing demands.

The software has been applied to DES science verification coadd images. Combined 
with the SExtractor software, GAIN provides a valued added catalog that can be used together with DES science verification coadd catalog. The value added catalog has been found useful in a variety of applications: testing photometric redshift precision with cluster galaxy members, measuring cluster central galaxy light profile, measuring cluster richness and finding strong lensing clusters etc. The cluster richness that counts the number of $L>0.2 L_{*}$ galaxies can change by up to $10 \%$ according to a preliminary study. Finding strong lensing clusters rely on identifying the faint blue lensing arcs at cluster center. The GAIN catalog may help increasing the number of strong lensing cluster candidates by up to $20 \%$.

\subsection{Summary}

Deep astronomical images face deblending challenges, especially in the crowded cores of galaxy clusters. Current deblending algorithms are not optimized to handle this problem. To take full advantage of the opportunity offered by new surveys like the DES, we need better methods for extracting accurate galaxy lists in cluster cores. In this chapter, we describe a relatively simple approach to sorting out blended sources in these crowded regions. The design of this GAIN package includes two innovative features.

1. This package makes use of the Laplacian of an intensity image for blended source detection. In deblending procedures, one of the biggest challenges occurs when the intensity contrast between blended sources is too low to trigger detection. In this chapter, we have shown that this problem can be alleviated by measuring the image intensity gradient. The image intensity gradient is often used in the computer vision field to bringing out fine details of an image. Future astronomical data production software can make use of this information to help deblending.

2. This package uses an interpolation technique to separate blended light from multiple sources. This is an improvement comparing to two popular approaches: simply assigning pixels to blended sources which is inaccurate but computationally efficient, and simultaneously fitting profiles of multiple sources which is accurate but computationally inefficient. Our method provides a nice balance between accuracy and efficiency.

We have tested this package on DES coadd images. Our tests show that it can increase the reliability of photometry for blended objects. It can also increase the completeness of blended source detection, while introducing only a modest number of spurious detections. 
The software has been applied to science verification data from DES. It is also possible to apply GAIN to HST and SDSS images. 


\section{CHAPTER 3}

\section{Evolution of Cluster Central Galaxies}

\subsection{Topic Introduction}

Bright central galaxies (BCGs) are the luminous elliptical galaxies residing at the centers of galaxy clusters or groups. Once commonly referred to as the brightest cluster galaxies, the name bright central galaxy better reflects their special nature as the central galaxy of a massive halo. BCGs are surrounded by a subsidiary population of satellite galaxies. Their centrality and large size sets them apart from the general galaxy population.

Early attention about BCGs started with studies about cD-type galaxies, since many BCGs are enveloped by extended stellar halos (Matthews et al., 1964). Statements that this population is not consistent with being statistically drawn from the global galaxy luminosity function led Tremaine \& Richstone (1977) to argue that BCGs require a special formation process. Analytical and early numerical estimates of their growth through dynamical friction and resultant cannibalism of cluster galaxies was soon identified as a viable process (Ostriker \& Tremaine, 1975; White, 1976; Hausman \& Ostriker, 1978; Richstone \& Malumuth, 1983). Early N-body simulations of merging pairs and groups of galaxies led Dubinski (1998) to perform the first N-body study of BCG formation in a massive halo formed within a cold dark matter (CDM) cosmology. In that study, growth through early merging of a few massive galaxies dominated over late-time accretion of many smaller systems.

The modern context of BCG assembly through hierarchical growth within an evolving spatial network of dark matter halos is now well established, but detailed understanding of various competing astrophysical processes remains elusive. Models in which BCGs accrete their stellar mass through "dry" merging with red and old galaxies produce scaling behavior and light profiles in fairly good agreement with observations (e.g., Ruszkowski \& Springel, 2009; Laporte et al., 2013).

Pure N-body models of dry merging ignore intra-cluster gas processes such as cooling 
and subsequent accretion and star formation of baryons onto the BCG. Semi-analytical models find that such cooling needs to be mitigated by heating, and AGN feedback in a socalled "radio mode" is proposed as the solution (Croton et al., 2006; De Lucia \& Blaizot, 2007). Simulations with explicit hydrodynamic treatment of the baryons are struggling to develop sub-grid models that capture the full complexity of the baryon behavior (e.g., Martizzi et al., 2012; Ragone-Figueroa et al., 2013; Martizzi et al., 2014; Pike et al., 2014).

While BCG in situ star formation is almost certainly suppressed by the quenching effect of AGN (active galactic nuclei) feedback (Fabian, 1994, 2012), observational studies have found that residual star formation of $\sim 10-100 M_{\odot} y \mathrm{r}^{-1}$ exists in many nearby BCGs (Fraser-McKelvie et al., 2014; Liu et al., 2012; Groenewald \& Loubser, 2014). A most puzzling study has observed a BCG starburst of $740 \pm 160 M_{\odot} \mathrm{yr}^{-1}$ in the $z=0.596$ Phoenix cluster (McDonald et al., 2012). Such a large star formation rate would contribute significantly to BCG stellar mass even if it lasted for just $1 \mathrm{Gyr}$.

Recent arguments based on local cooling-to-dynamical timescales tie together this rich phenomenology in a self-regulated precipitation model (Voit et al., 2015, and references therein). Idealized hydrodynamic simulations (Li \& Bryan, 2014a,b; Meece et al., 2015) support an episodic picture in which gas below a cooling threshold (roughly $t_{\text {cool }} / t_{\text {dyn }}<10$ ) feeds black hole accretion and local star formation, with AGN feedback serving as the rectifier that shuts down cooling and allows the cycle to refresh. With $H S T$ observations of BCGs in the CLASH sample, Donahue et al. (2015) offer evidence that ultraviolet morphologies and star-formation rates of BCGs in CLASH clusters display features remarkably similar to those anticipated by these simulations.

The semi-analytical expectations of BCG growth have been called into question by a number of observations that report significantly slower build-up of stellar mass over time (Whiley et al., 2008; Collins et al., 2009; Lidman et al., 2012; Lin et al., 2013b,b; OlivaAltamirano et al., 2014; Inagaki et al., 2015). This tension highlights limitations in our current understanding of BCG formation and motivates this work.

The production of intra-cluster light (ICL) is another important process affecting BCG formation over time. The ICL contains stars that got dispersed into intracluster space from BCGs or BCG mergers (see: Contini et al., 2014). Simulation and observational studies show that ICL can make up 5-50\% of the total cluster/group stellar content (Zibetti et al., 2005; Krick et al., 2006; Krick \& Bernstein, 2007; Gonzalez et al., 2007; Toledo et al., 2011; Guennou et al., 2012; Burke et al., 2012; Montes \& Trujillo, 2014; Giallongo et al., 2014; Presotto et al., 2014; Burke et al., 2015). Details of how the ICL is formed and how its properties might vary from cluster to cluster remain unsettled (Monaco et al., 2006; Conroy et al., 2007; Puchwein et al., 2010; Rudick et al., 2011; Cui et al., 2014; Contini 
et al., 2014; DeMaio et al., 2015).

To advance our understanding about the above processes and BCG formation in general, it is important that we continue to refine our measurements of BCG growth. Most upto-date observations are yielding perplexing or even contradictory results on this subject, perhaps because of in-comparability in their processing BCG observables (Mandelbaum et al., 2005; Lauer et al., 2007; Bernardi et al., 2007). For instance, a few studies based on high redshift ( $\mathrm{z}_{i}$ 1.0) X-ray selected clusters (Stott et al., 2010, 2011; Collins et al., 2009; Whiley et al., 2008) finds no sign of BCG stellar mass growth, while others based on clusters at low and high redshifts do observe the change (Brough et al., 2002; Lin et al., 2013b; Lidman et al., 2012, which included some of the samples from the forementioned X-ray studies). On the other hand, deriving BCG luminosity and hence BCG stellar mass from imaging data is not straightforward, and inconsistent measurements may have affected many previous findings about BCG formation. Finally, BCG mass is known to be correlated with cluster mass, which needs to accounted for when studying the change of BCG mass over time (see for example, Lidman et al., 2012). Advances in our understanding of the nature of the growth of BCGs require a careful accounting of all of the ingredients, including their measurement uncertainties.

In this work, we investigate BCG stellar mass growth using DES Science Verification (DES SV) data, and a new sample of 106 X-ray selected clusters and groups from the DES $X M M$ Cluster Survey (XCS), an XMM-Newton archival discovery project. Through using this X-ray selected sample, selection effect on studying BCG's optical properties are greatly alleviated: X-ray selected clusters display a wider variety of optical properties compared to optically selected clusters (Harrison et al., 2012). The cluster and group sample spans a redshift range of $[0,1.2]$, and a mass range of $\left[3 \times 10^{13} M_{\odot}, 2 \times 10^{15} M_{\odot}\right]$. While most previous studies on this redshift range or cluster mass range are combining different samples or different imaging data sets, we study a single cluster sample with the deep optical data from DES. In this work, we also pay particular attention to possible biases affecting BCG photometry, and have carefully evaluated the uncertainties associated with cluster mass, redshift, BCG luminosity and BCG stellar mass measurements.

The rest of this chapter is organized in the following order. From Section 3.2 to 3.5, we present our data sets and derive measurements about cluster masses, BCG luminosities and BCG stellar masses. We perform a matching exercise of BCG redshift evolution to the Millennium Simulation expectations in Section 3.6, then fit both simulated and observed BCG populations to a simple low-order model in Section 3.7. We compare this model to previous estimates of BCG growth rate in Section 3.8. We summarize our results in Section 4.8. Throughout this chapter, we assume $\Omega_{m}$ to be $0.3, \Omega_{\Lambda}$ to be 0.7 , and the 
Hubble parameter $h$ to be 0.7 .

\subsection{Data}

This work is based on an X-ray selected cluster and group sample from the DES-XCS project. BCG photometry is derived from DES Science Verification data. The rest of this section introduces the DES SV data, the DES-XCS sample, and the BCGs selection procedure.

\subsubsection{DES Science Verification Data}

The Dark Energy Survey is a ground-based optical survey that uses the wide-field DECam camera (Flaugher et al., 2015a) mounted on the 4m Blanco telescope to image 5,000 deg ${ }^{2}$ of the southern hemisphere sky (Sánchez, 2010). The work is based on $200 \mathrm{deg}^{2} \mathrm{DES}$ Science Verification (SV) data. This data set was taken during the 2012B observing season before the main survey (Diehl et al., 2014) began. A large fraction of the SV data have full DES imaging depth (Lin et al., 2013a) and are processed with the official DES data processing pipeline (Mohr et al., 2012). A more detailed review can be found in Sánchez et al. (2014).

\subsubsection{The DES-XCS Cluster and Group Sample}

The XMM Cluster Survey serendipitously searches for galaxy cluster (and group) candidates in the XMM-Newton archive (Lloyd-Davies et al., 2011; Mehrtens et al., 2012; Viana et al., 2013). The cluster candidates are then verified with optical/infrared imaging data, which confirm the existence of red sequence galaxies. Photometric redshifts of the confirmed clusters are also subsequently derived with the red sequence locus. Using DES SV data, Miller et al. (in prep; referred to as M15 in the rest of the chapter) have identified $\sim 170$ X-ray selected clusters and groups from XCS. M15 also measures their photometric redshifts and verify the measurements against archival spectroscopic redshifts ${ }^{1}$. In this work, we use a sub-sample from M15 that consists of 106 clusters and groups with mass above $3.0 \times 10^{13} \mathrm{M}_{\odot}$. These clusters and groups are all referred to as "clusters" in the rest of the chapter. In Figure 3.1, we show their mass and redshift distribution. For comparison, we also show the mass and redshift distribution of the cluster sample used in a similar

\footnotetext{
${ }^{1}$ http://ned.ipac.caltech.edu
} 


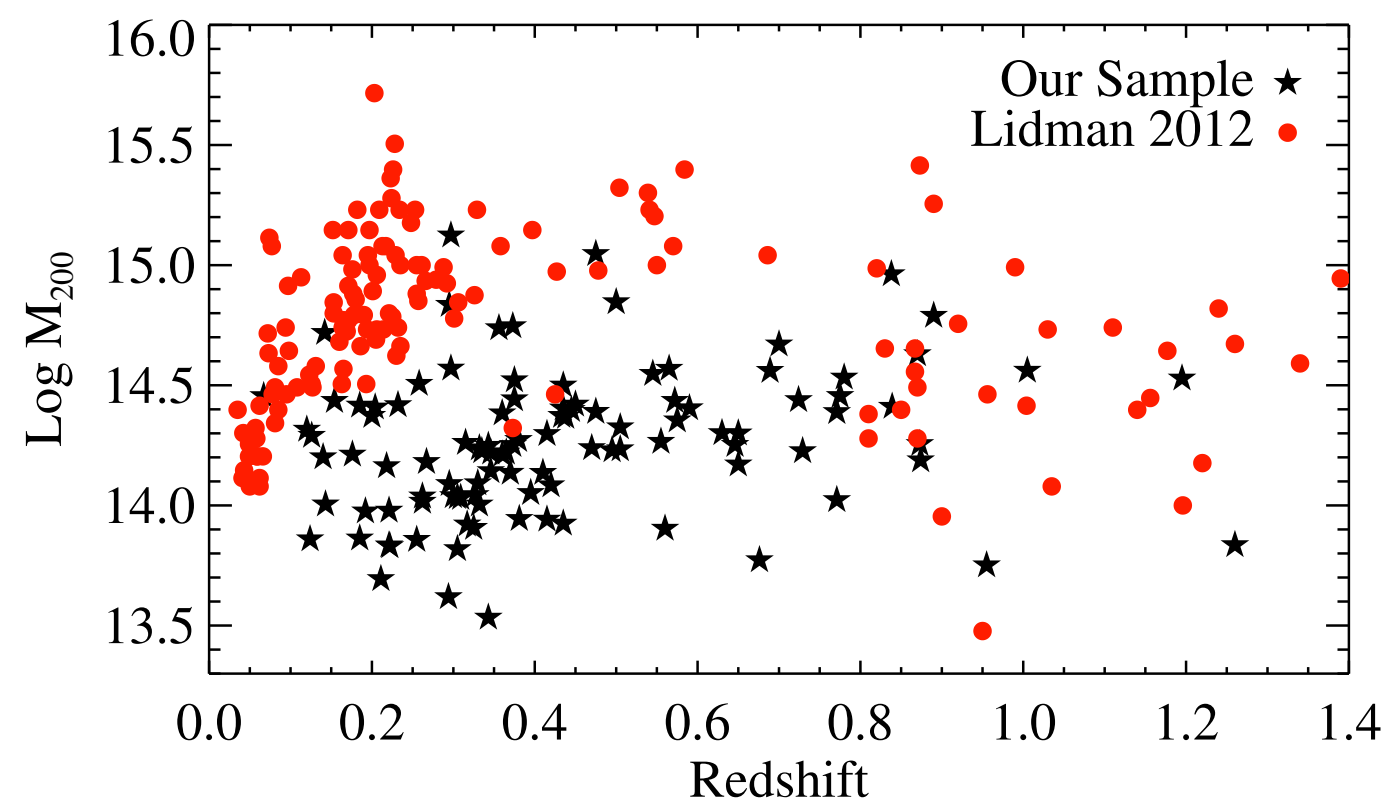

Figure 3.1: The mass and redshift distribution of the DES-XCS sample (black stars) compared to that of Lidman et al. (2012, red circles). 


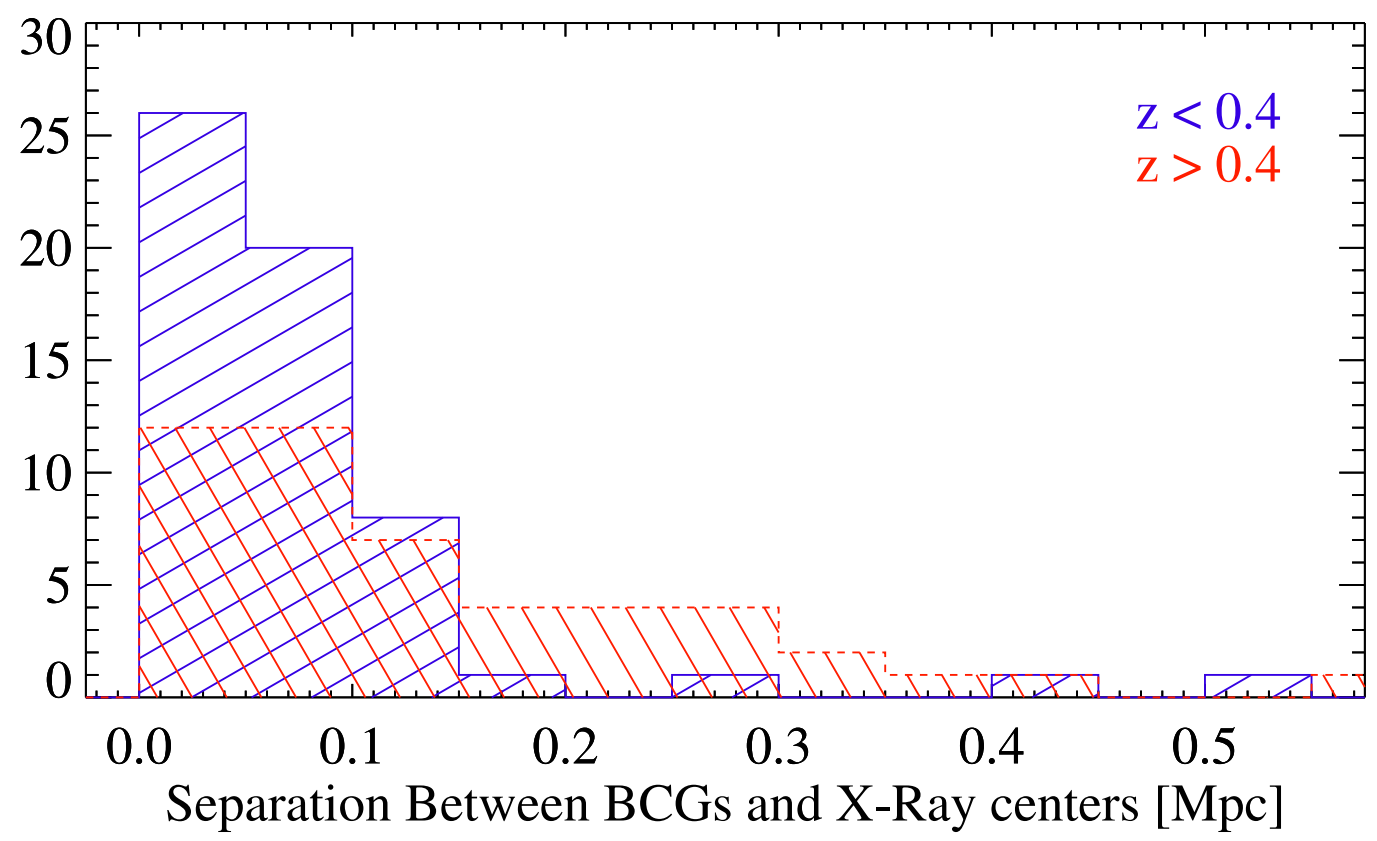

Figure 3.2: Distances between the BCGs and the X-ray emission centers for our cluster sample. Half of the BCGs are separated less than $0.07 \mathrm{Mpc}$ (transverse comoving distance with negligible uncertainties from redshifts measurements) from the X-ray centers, and the large separations $(>0.4 \mathrm{Mpc})$ happen in clusters that may not be relaxed or appear to have spurious foreground/background emissions.

study (Lidman et al., 2012). Our sample covers a lower mass range, and appears to be more evenly distributed in the redshift-mass space.

We note that a handful of the clusters do not seem to have significant galaxy overdensity associated with them. It is possible that our sample contains spurious clusters which originates from foreground/background X-ray contaminations. We have re-analyzed our analysis after removing 8 clusters that are not associated with significant galaxy overdensity. The results are consistent with those presented in this chapter within $0.5 \sigma$. Given that these 8 clusters are in the low mass range (generally below $10^{14} M_{\odot}$ ), removing them may introduce an artificial mass selection effect. We therefore do not attempt to do so in this work. We also note that other factors, including BCG photometry measurement and cluster mass scaling relations at the low mass end (see discussion in Section 3.7.3 and 3.8.2), have bigger effect on our results than the possible spurious clusters in the sample. 


\subsubsection{BCG Selection}

The BCGs are selected through visually examining the DES optical images, the X-ray emission contours, and the galaxy color-magnitude diagram. In this procedure, we aim to select a bright, extended, elliptical galaxy close to the X-ray emission center, which also roughly lies on the cluster red sequence. If there exist several red, equally bright and extended ellipticals close to the X-ray center, we select the nearest one. We did not notice a proper BCG candidate with a blue color.

We check our visual BCG selection against the central galaxy choice of a preliminary version of the DES SV RedMaPPer cluster catalog (see the algorithm in Rykoff et al., 2014). Out of the 106 XCS clusters and groups, 64 are matched to RedMaPPer clusters and the majority (61) identify the same BCG. In the cases where we disagree with the BCG, we choose the brighter, more extended galaxy closest to the X-ray center while RedMaPPer selects a galaxy further away. The other 42 non-matches are caused by the different data coverage, redshift limit, and mass selection of the two catalogs: the RedMaPPer catalog employs only a subset of the SV data to achieve relatively uniform depth for selecting rich clusters below redshift 0.9 .

In Figure 3.2, we show the distance distribution between the selected BCGs and the Xray emission centers. Half of the BCGs are separated by less than $0.07 \mathrm{Mpc}$ (comparable to Lin \& Mohr, 2004) from the X-ray emission centers, regardless of the redshifts of the clusters.

\subsection{Galaxy Cluster Mass}

The cluster mass $\left(M_{200}\right.$, the mass inside a 3D aperture within which the averaged matter density is 200 times the critical density) is either derived with X-ray temperature or Xray luminosity, using a lensing calibrated $M-T$ relation (Kettula et al., 2013). Because $\mathrm{XCS}$ is a serendipitous survey, not all the clusters have high quality X-ray temperature measurements. For these clusters, we derive their masses from X-ray luminosity. Further details about this procedure.

\subsubsection{Cluster Mass From X-ray Temperature}

We use a lensing calibrated $M-T$ scaling relation from Kettula et al. (2013) to derive cluster mass from X-ray temperature ( $T_{X}$, core not excised). In Kettula et al. (2013), weak lensing mass measurements are obtained for 10 galaxy groups in the mass range $0.3-$ $6.0 \times 10^{14} h_{70}^{-1} M_{\odot}$. Together with 55 galaxy clusters (many above $2 \times 10^{14} h_{70}^{-1} M_{\odot}$ ) from 

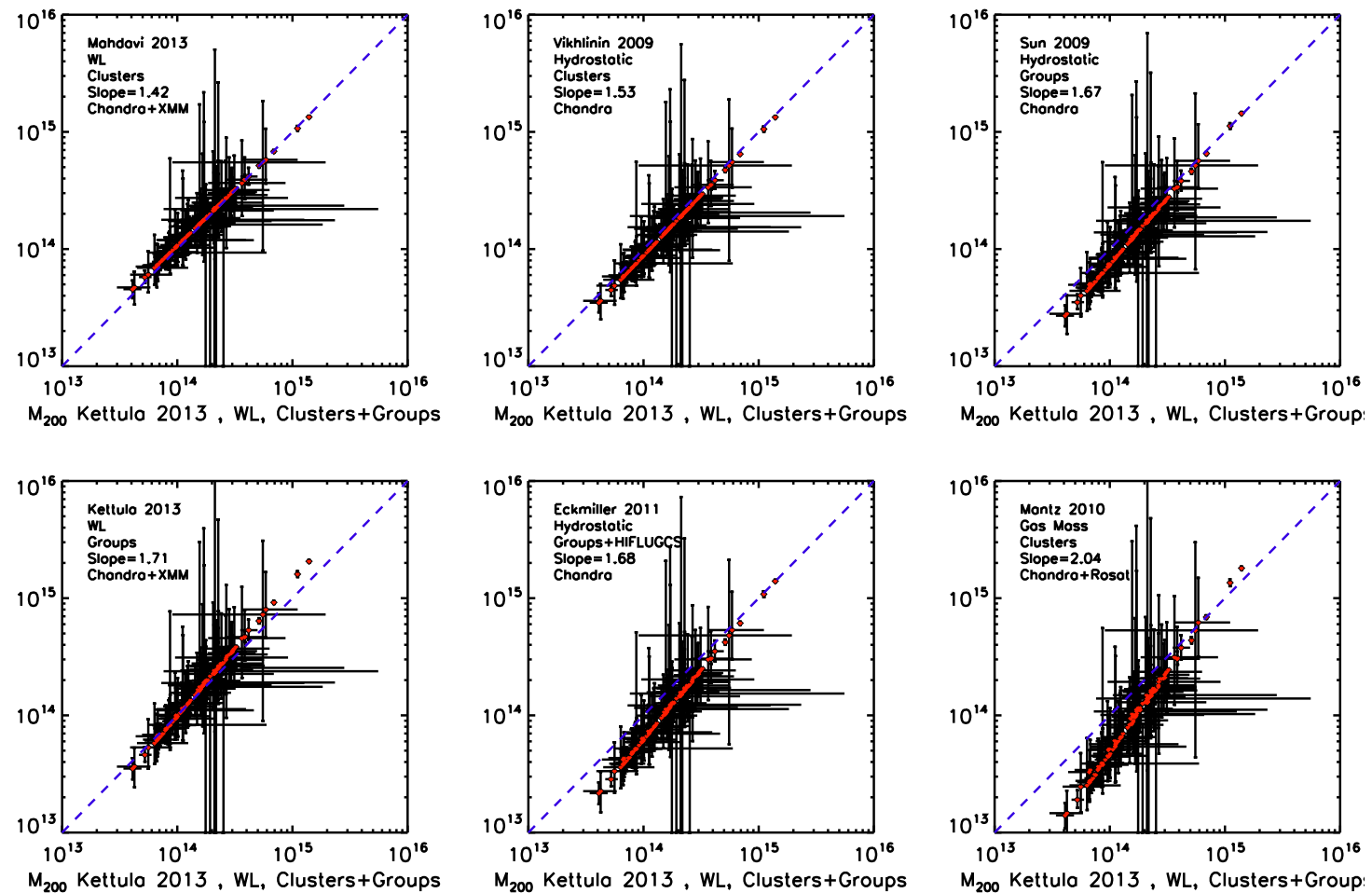

Figure 3.3: Comparison of the cluster masses derived from a few $M-T$ scaling relations. We plot the mass derived from the Kettula et al. 2013 relation on the $x$-axis of all the panels. The Kettula et al. $2013 M-T$ scaling relation agrees well with other relations at the cluster scale (See Section 3.3.1). For simplification, we only include X-ray temperature measurement uncertainty in this figure. 

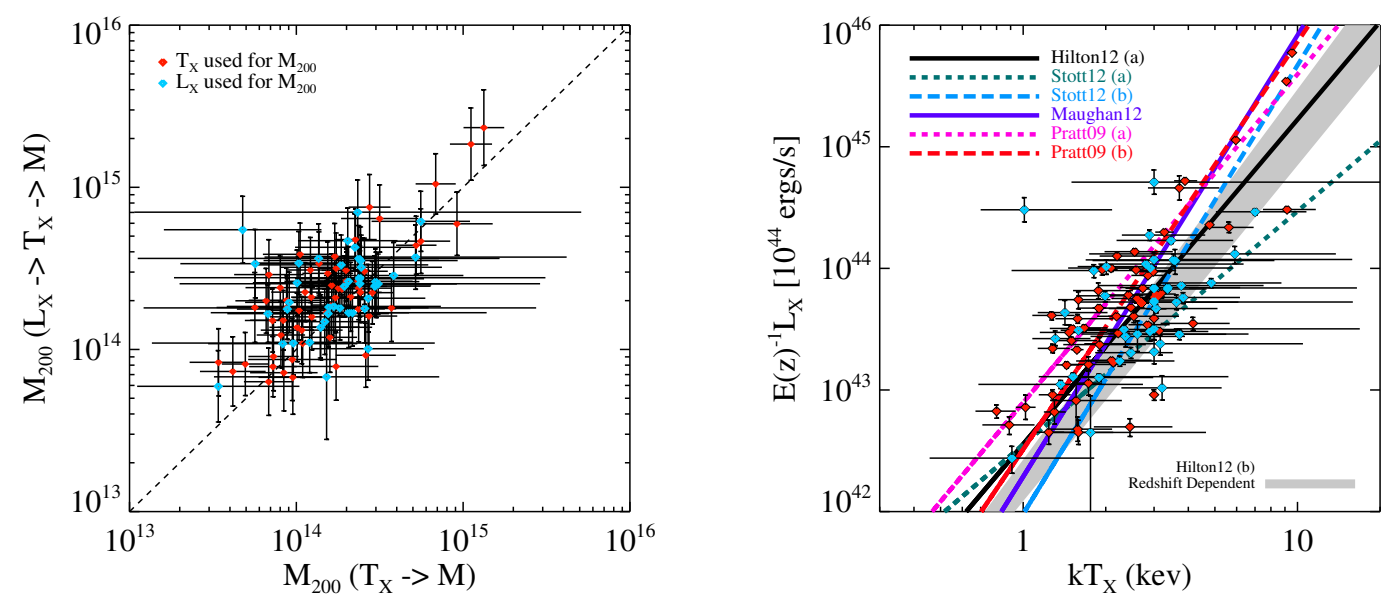

Figure 3.4: Left: We derive cluster masses from both X-ray luminosities and X-ray temperatures, and decide which one to use through comparing their uncertainties. Right: X-ray temperature and X-ray luminosity of the XCS clusters plotted against a few scaling relations in literature.The grey band shows the redshift-dependent $L-T$ relation in Hilton et al. 2012 between $\mathrm{z}=1$ and $\mathrm{z}=0$.

Hoekstra et al. (2011, 2012); Mahdavi et al. (2013), Kettula et al. (2013) derive weak lensing calibrated $M-T$ relation across the group to cluster range ${ }^{2}$.

We check the Kettula et al. (2013) $M-T$ scaling relation against a few other studies based on the gas content (Figure 3.3). The Hydrostatic Equilibrium (HSE, Sun et al., 2009; Eckmiller et al., 2011; Vikhlinin et al., 2009) and gas mass fraction (Mantz et al., 2010) calibrated relations agree with the Kettula et al. (2013) $M-T$ relation at the cluster scale, but have troubles matching to it at the group scale (Sun et al., 2009; Eckmiller et al., 2011). As known from simulations (Nagai et al., 2007; Rasia et al., 2012, see Kettula et al. (2013) for discussion), the disagreement is not surprising as HSE masses are biased low at the group scale and the gas mass fraction relation is only derived with the most massive clusters.

We use the Kettula et al. (2013) scaling relation to derive cluster $M_{500}$ from X-ray temperature, and then the $\mathrm{Hu} \&$ Kravtsov (2003) relation to derive $M_{200}$ from $M_{500}$. We assume the cluster concentration parameter to be 5 in this procedure. Using a different concentration parameter in the $[3,5]$ range only changes $M_{200}$ at percent level. We also assume the intrinsic scatter of $M_{500}$ to be $0.1 \mathrm{dex}$ as typically found in simulation studies

\footnotetext{
${ }^{2}$ Chandra temperatures in Mahdavi et al. (2013) are adjusted to match XMM calibration.
} 
(Kravtsov et al., 2006; Henry et al., 2009).

\subsubsection{Cluster mass from X-ray Luminosity}

We resort to X-ray luminosity ( $L_{X}$, core not excised) to estimate masses for clusters/groups that do not have high quality temperature measurements. We first derive $\mathrm{X}$-ray temperature using a $L-T$ scaling relation, and then derive $M_{200}$ using the procedure above.

We use a self-similar, redshift-dependent $L-T$ scaling relation from Hilton et al. (2012), but also experimented with a few other self-similar $L-T$ relations (see Figure 3.4) from Stott et al. (2012); Maughan et al. (2012); Pratt et al. (2009). The Hilton et al. (2012) relation, which is also based on a XCS sample (Mehrtens et al., 2012), provides the best fit to our data. We assume 0.1 dex intrinsic scatter for the derived temperatures, as it is constrained in Hilton et al. (2012).

\subsubsection{Mass Uncertainties and the Choice between Temperature and Luminosity Based Masses}

We decide between $L_{X}$ and $T_{X}$ based masses through comparing their uncertainties. To estimate the mass uncertainties associated with each method, we produce 200 "pseudomeasurements" for each cluster, sampling through temperature/luminosity measurement uncertainty, the scaling relation uncertainty, and the intrinsic scatter of the relations. We derive mass uncertainties for $L_{X}$ or $T_{X}$ based masses assuming log-normal distribution for the 200 "pseudo-measurements". If the uncertainty of $T_{X}$ mass is larger than the uncertainty of $L_{X}$ mass by 0.05 dex (we prefer $T_{X}$ mass since $L_{X}$ mass is more susceptible to biases), we use $L_{X}$ mass in lieu of $T_{X}$ mass. In the end, about half of the cluster masses are derived with $L_{X}$, and most of the clusters masses have $<0.25$ dex uncertainty.

The 200 "pseudo-measurements" are also used to derive the $M_{200}$ covariance between the cluster sample. Because we are including scaling relation uncertainty, the $M_{200}$ covariance matrix is not diagnonal.

\subsection{BCG Photometry}

Measuring BCG photometry is among the most controversial topics in BCG studies. We use magnitude measured with circular apertures of $15 \mathrm{kpc}, 32 \mathrm{kpc}, 50 \mathrm{kpc}$ and $60 \mathrm{kpc}$ radii. The main results are derived with the $32 \mathrm{kpc}$ radius apertures, considering the BCG half light radius measurements in Stott et al. (2011). 
Top Row: Kron Magnitude
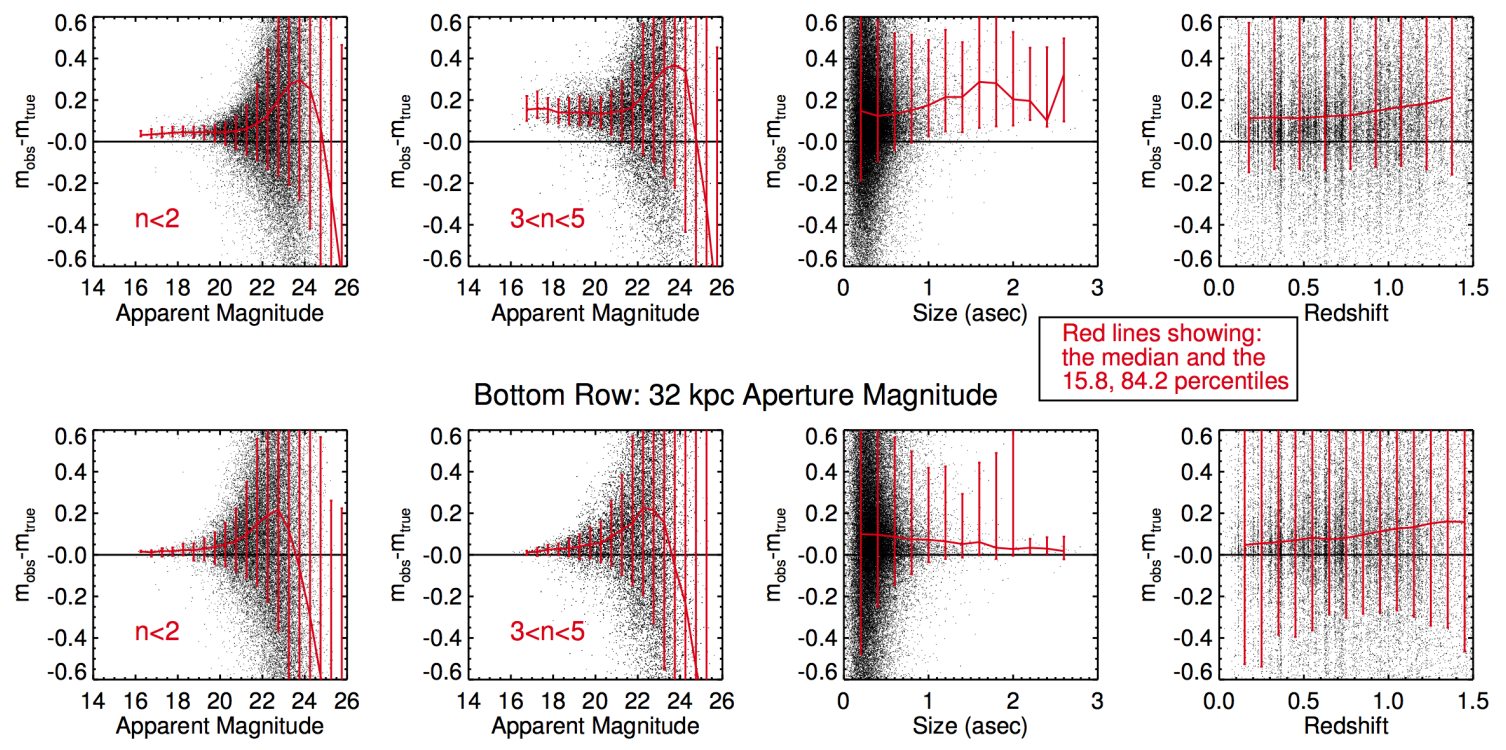

Figure 3.5: We investigate measurement bias associated with Kron magnitude and aperture magnitude using the UFIG sky simulation (see Section 3.4.4 for details). Note that this test is done for a general galaxy population rather than BCGs. BCGs below redshift 1.0 generally have Sersic index $>2$ and apparent magnitude below 22. In the top row, $m_{\text {true }}$ is the galaxy's input total magnitude, but in the bottom row, $m_{\text {true }}$ is the galaxy's input $32 \mathrm{kpc}$ aperture magnitude. To summarize this figure, Kron magnitude tend to under-estimate the brightness of bulge-like galaxies and extended galaxies, while aperture magnitude remain well-behaved for galaxies of all profiles and sizes. The measurements from both systems do become biased for faint galaxies with apparent magnitude above 23, but the bias is unimportant for this work. For efficiency, we use SExtractor output in this comparison, which matches our own measurements for a general galaxy population. 
(a)

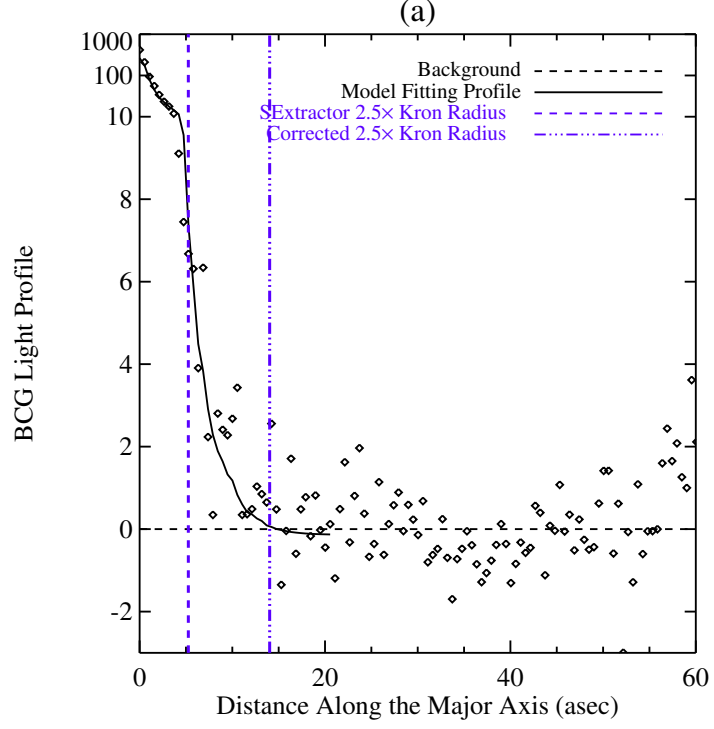

(b)

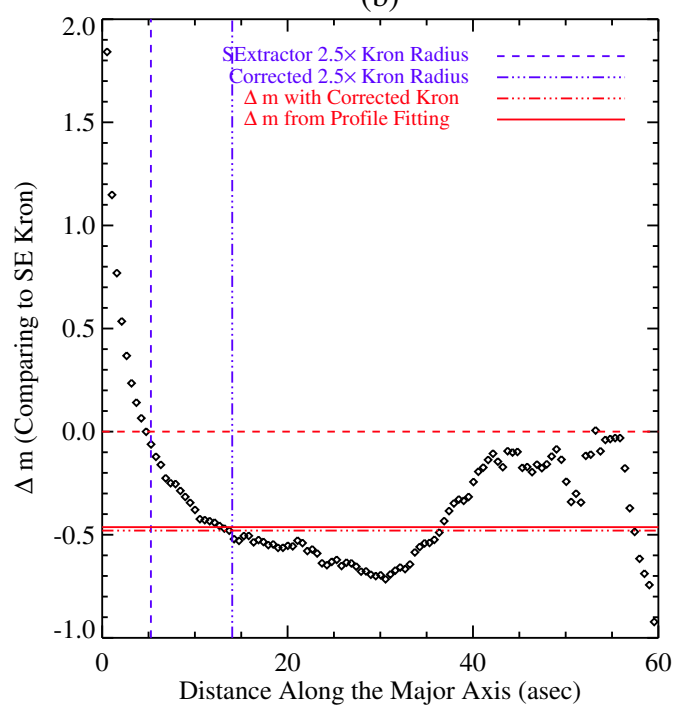

Figure 3.6: The popular SExtractor software tends to under-estimate BCG Kron radius, resulting in significant brightness under-estimation. In panel (b), we show the difference in Kron magnitude measurement, $\Delta m$, when the measurement is made to a different Kron radius ( $x$-axis).

(a)

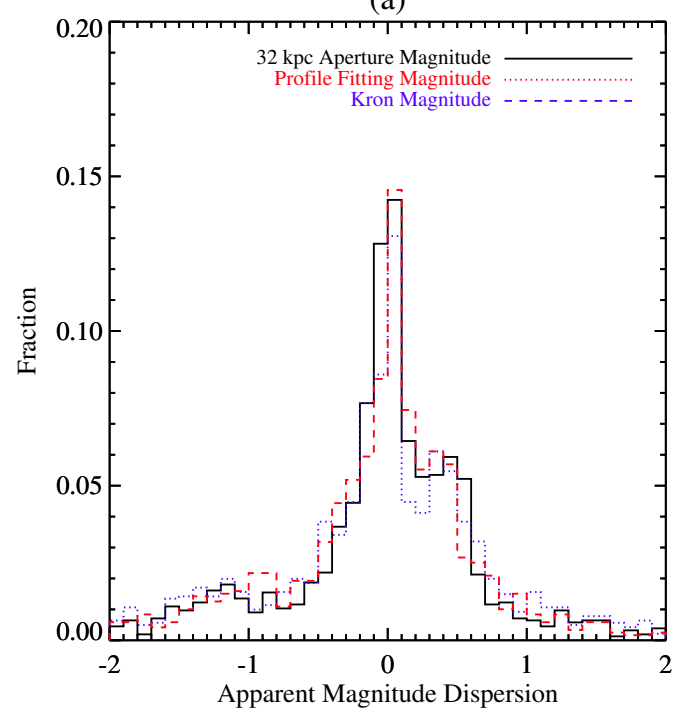

(b)

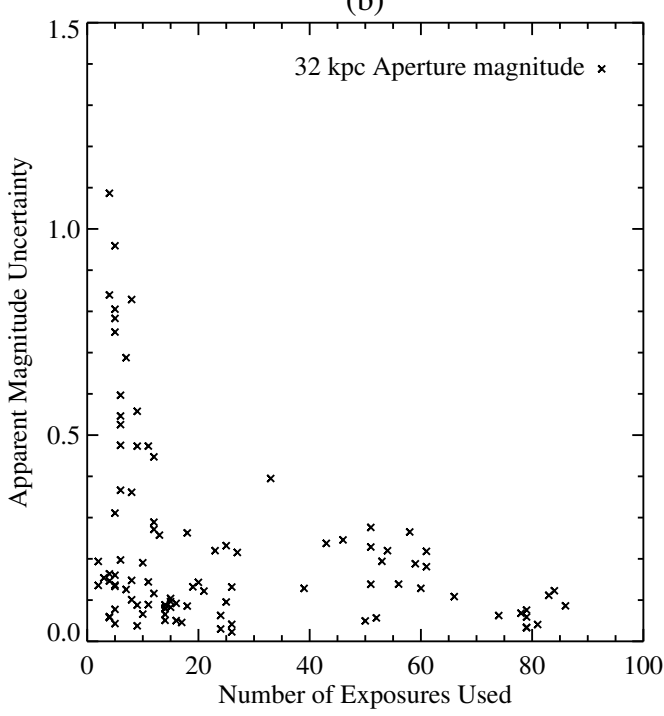

Figure 3.7: (a): Measurement dispersion of aperture magnitude, profile fitting magnitude and Kron magnitude. (b): We sample BCG apparent magnitude from multiple exposures to evaluate the measurement uncertainty, and our measurement accuracy is limited by the number of exposures we have. 
BCG photometry measurement is based on products from the official DES Data Management (DESDM) processing pipeline (Mohr et al., 2012). In this pipeline, single exposure images are processed, calibrated and later background-subtracted and co-added to produce coadd images. DESDM also runs an advanced version of SExtractor (Bertin, 2011; Bertin \& Arnouts, 1996) on processed single exposure images and coadd images to produce star/galaxy catalogs, which we do not use because of existing problems for BCGs. In this chapter, we derive BCG photometry from processed single exposure images. The following Sections 3.4.1 to 3.4.4 describe our explorations on measuring BCG flux with different magnitude conventions. We discuss potential problems associated with Petrosian magnitude, Kron magnitude, profile fitting magnitude, and aperture magnitude here, but most of the problems are already well analyzed in literature (especially, see Graham \& Driver, 2005; Häussler et al., 2007; von der Linden et al., 2007; Bernardi et al., 2014). Nevertheless, we provide a summary in this Section.

\subsubsection{Petrosian Magnitude}

Petrosian magnitude measures the flux enclosed within a scaled aperture known as the "Petrosian radius", which is calculated considering background noise level and object light profile (Petrosian, 1976; Blanton et al., 2001; Yasuda et al., 2001). It is extraordinarily robust under exposure to exposure variations, but not appropriate for extended galaxies. Although Petrosian magnitude accounts for most of the flux of a disk-like (Sersic index = 1) galaxy, it will only recover $80 \%$ of the flux for a bulge-like galaxy with a de Vaucouleurs (Sersic index $=4)$ profile (Blanton et al., 2001).

Indeed, a series of studies have found that using Petrosian magnitude (see Bernardi et al., 2013; He et al., 2013, for relevant discussion), the brightness of Luminous Red Galaxies (LRGs) is under-estimated by about $0.3 \mathrm{mag}$. Moreover, the missing flux problem is sensitive to the profiles of extended galaxies, and worsens quickly with higher Sersic index. Graham \& Driver (2005) estimate that Petrosian magnitude at its most popular configuration (one that is adopted by SDSS) under-estimates the luminosity of Sersic index $=10$ galaxies by $44.7 \%$ (0.643 mag)! For this reason, we are not exploiting Petrosian magnitude in this work.

\subsubsection{Kron Magnitude}

Kron magnitude is another scaled aperture magnitude, measuring the flux enclosed within a few "Kron radius" (usually 2.5 Kron radius), and the Kron radius is decided from the light profile (Kron, 1980). Kron magnitude is not as robust as Petrosian magnitude under 
exposure to exposure variations, but does appear to be more proper for extended galaxies.

Like Petrosian magnitude, Kron magnitude recovers most of the flux of a disk-like galaxy, but misses $10 \%$ of the flux for a bulge-like galaxy (Sersic index $\sim 4$, Andreon, 2002; Graham \& Driver, 2005). Unlike Petrosian magnitude, the flux missing ratio is insensitive to the galaxy Sersic index. Graham \& Driver (2005) estimate that the missing flux varies only at percent level when Sersic index changes from 2 to 10. Indeed, tests with simulated skies (see Andreon, 2002, or our test in Figure 3.5) show that Kron magnitude only underestimates the brightness of bulge-like galaxies by about $0.2 \mathrm{mag}$. It also appears to be indifferent to the presence of ICL: when we apply the measurement to simulated BCGs enclosed by ICL (we use the model in Giallongo et al., 2014), the measurement changes only $<\sim 0.1 \mathrm{mag}$.

As proper as the design of Kron magnitude seems to be, the real problem comes from observationally deriving the Kron radius. As pointed out in Graham \& Driver (2005), correctly estimating Kron radius requires integration over the light profile to a very large radius, usually many times the half light radius for extended galaxies. If the integration is improperly truncated, the measured Kron radius will be much smaller, and Kron magnitude turns out to be catastrophically wrong - it may under-estimate the flux of an extended galaxy by as much as $50 \%$ (Bernstein et al., 2002)!

We find this to be a frequent problem for BCG measurements from the widely-used SExtractor software (i.e., mag_auto), as demonstrated in Figure 3.6 (a) and (b). The Kron radius from SExtractor is two times smaller than it should be for one of the BCGs, and the BCG light intensity at 2.5 SExtractor Kron radius is still high. As a result, SExtractor underestimates the Kron flux of this BCG by $\sim 0.5$ mag. This problem seems purely algorithmic though. Using the galaxy intensity profile to re-calculate Kron radius until it converges, we are able to correct this measurement error. Comparing the corrected measurements to the magnitude measurements from profile fitting (see Section 3.4.3), we recover the 0.2 mag accuracy of Kron magnitude as discussed above.

For this work, we have re-done our analysis using Kron magnitude. We re-compute the Kron radius instead of using SExtractor output, but the result remains qualitatively similar.

\subsubsection{Profile Fitting Magnitude}

We have also experimented with BCG profile fitting magnitude from the GALFIT software (Peng et al., 2002, 2010a). We fit the BCGs with a model consisting two Sersic profiles, one with Sersic index $=1$ (i.e., a disk profile) and one with flexible Sersic index as suggested by Bernardi et al. (2014); Meert et al. (2015). We convolve these models to point spread 
functions (PSF) derived with the PSFex software (Bertin, 2011), and carefully mask all neighboring objects including blended objects identified with the GAIN deblender (Zhang et al., 2015b). Overall, the design of this procedure is similar to the Galapagos fitting software (Barden et al., 2012).

For this work, we only use the profile fitting magnitude for testing purpose (see Section 3.4.2 and Section 3.4.4). We hesitate about using it for scientific purpose as we realize that the measurement needs to be extensively tested with sky simulations as in Häussler et al. (2007); Bernardi et al. (2014); Meert et al. (2015). Upon evaluating the profile fitting magnitude uncertainties (see Figure 3.6), we do not find it to improve BCG measurement accuracy and therefore do not consider the testing efforts to be worthwhile for this work. We nevertheless have re-done our analysis using this magnitude, but the result remains qualitatively similar.

\subsubsection{Aperture Magnitude Used in this Work}

In this work, we measure BCG magnitude with circular apertures of $15 \mathrm{kpc}, 32 \mathrm{kpc}, 50$ $\mathrm{kpc}$, and $60 \mathrm{kpc}$ radii. The main results in this work are derived with the $32 \mathrm{kpc}$ measurements, considering the BCG half light radius measurements in (Stott et al., 2011). The 32 kpc aperture choice is also comparable to the popular Kron magnitude aperture (2.5 Kron radius) measurements from the SExtractor software. We carefully mask BCG neighbors (including blended objects identified with the GAIN deblender, Zhang et al., 2015b) and interpolate for the BCG intensity in the masked area. To realistically evaluate BCG magnitude uncertainty, we perform the procedure on processed single exposure images, use the median as the measurement, and evaluate the uncertainty through bootstrapping. We find our typical measurement uncertainty to be $\sim 0.4 \mathrm{mag}$, significantly larger than the SExtractor estimation from co-added images (but not larger when we bootstrap the SExtractor measurements from single exposure images). Since we perform the measurements independently on different exposures, our uncertainty is more comprehensive than the SExtractor uncertainty from co-added images (also see the magnitude measurement scatter test in Figure 3.5). Our measurement becomes uncertain when we have few exposures to work with (see Figure 3.6), which will be improved as DES assembles more exposures in the coming years.

To evaluate the sky background level around BCGs, we use background check maps generated with the SExtractor software from DESDM, configured with the "Global evaluation" process. We sample the values in a ring with inner and outer radius of $\sim 13$ arcsec and 18 arcsec from the BCG. We have investigated how sky background estimation af- 
Table 3.1: Parameters of the stellar population models

Formation Redshift

Metallicity

E-folding time (Gyrs)

Observed Redshift
$20,10,8,5,4,3,2.5,2,1.5,1.0,0.8,0.5$

$0.03,0.025,0.02,0.015,0.01,0.008,0.005,0.003,0.002$

$30,15,10,8,5,3,2,1,0.8,0.5,0.3,0.1$

$1.50,1.49,1.48, \ldots, 0.03,0.02,0.01$

fects our measurement, as it was considered a difficult task for BCGs. We find it to have only marginal influence. Indeed, even by using a "Global" setting, SExtractor still overestimates the background around some extremely bright sources, known as the dark halo problem within DES (after background subtraction, the light intensity of a bright object falls slightly below 0 at the outskirt). However, changing the background sampling location only marginally shift our final measurements. In fact, other details of the measurement procedure, like in-complete masking of neighboring sources may cause bigger problems.

We test for measurement bias associated with aperture magnitude and Kron magnitude with simulated DES images, using the UFIG simulation (Bergé et al., 2013; Chang et al., 2014). This sky simulation is based on an N-body dark matter simulation populated with galaxies using the Adding Density Determined GAlaxies to Lightcone Simulations (ADDGALS) algorithm (Wechsler, in prep.; Busha, in prep.; Dietrich et al., 2014, for a review). We find that both aperture magnitude and Kron magnitude tend to under-estimate the brightness of fainter sources, but the effect is negligible for even the furthest BCGs $(z$ band apparent magnitude is about 22). In addition, the bias would only have suppressed the significance of our result, as further objects are evaluated to be less massive/luminous. We also perform the test with sky simulations based on adding simulated galaxies into real DES co-add images, known as the Balrog simulation (Suchyta et al., 2015), and came to the same conclusion.

\subsection{BCG Luminosity and Stellar Mass}

We correct for galactic extinction using the stellar locus regression method (High et al., 2009; Kelly et al., 2014; Rykoff et al., in prep.), and compute BCG luminosities and stellar masses using the stellar population modeling technique. We use the best-fit model to compute the K-correction factor and the mass-to-light ratio.

The procedure begins with using the EZGal package (Mancone \& Gonzalez, 2012), the Chabrier (2003) Initial Mass Function (IMF) and the Conroy et al. (2009); Conroy \& Gunn (2010) simple stellar population (SSP) models to produce stellar population templates with various star formation histories and metallicities. We make templates with exponentially- 
decaying star formation histories (the $\tau$ model) characterized by various e-folding time, metallicity, formation redshift and observed redshift. In Table 3.1, we list the parameter values used for these templates.

We use a Chi-Square minimizing technique (see: Mitchell et al., 2013) to decide the stellar population template for each BCG. The fitting procedure is done with BCG photometry in DES $g, r, i, z$ bands and we fit the BCGs only to templates of their observed redshifts. After a best fit is identified for each BCG, we use the K-correction term from the template to compute BCG luminosity, and then the mass-to-light ratio to compute BCG stellar mass. We derive BCG luminosity in DES z band, and BCG stellar mass according to $z$ band luminosity. As an alternative, we also use the Blanton \& Roweis (2007) $\mathrm{K}$-correction package to derive BCG luminosity, but the result remains unchanged.

We then evaluate uncertainties associated with BCG apparent magnitude, redshift, and BCG mass-to-light ratio.

\subsubsection{BCG Luminosity Uncertainty}

We estimate BCG luminosity uncertainty combining BCG magnitude and redshift (see M15) uncertainties. To simplify subsequent analyses, we assume the redshift uncertainty to be statistical (systematic uncertainty is about $\sim 0.001$, comparing to $\sim 0.05$ statistical uncertainty, see M15). The redshift uncertainty is taken as 0.001 if archival spectroscopic redshift is available. We ignore $\mathrm{K}$-correction uncertainty as it is well decided.

\subsubsection{BCG Mass-to-Light Ratio Uncertainty}

We estimate BCG stellar mass uncertainty combining BCG luminosity uncertainty and BCG mass-to-light ratio (MLR) uncertainty. In this section, we pay special attention to estimating the MLR uncertainty from modeling star formation histories, which is the uncertainty from fitting a $\tau$-model to BCGs that formed through merging with galaxies of various star formation histories. We evaluate the uncertainty by applying the stellar population fitting procedure to DL07 BCGs (selected in Section 3.6.1). We compare the derived BCG MLR to their true values in the simulation.

In the left panel of Figure 3.8, we show the difference between the derived and true MLR plotted against redshift. We notice a systematic uncertainty of $\sim 0.05$ dex, likely caused by the mismatch of IMF in our procedure and in DL07 (a Chabrier IMF produces a mass-to-light ratio 0.05 dex higher than that of a Kroupa IMF, see Papovich et al., 2011).

We also notice a statistical scatter with the derived values, ranging from 0.05 dex to 0.1 dex with weak dependence on redshift (Figure 3.8), but no dependence on cluster mass or 

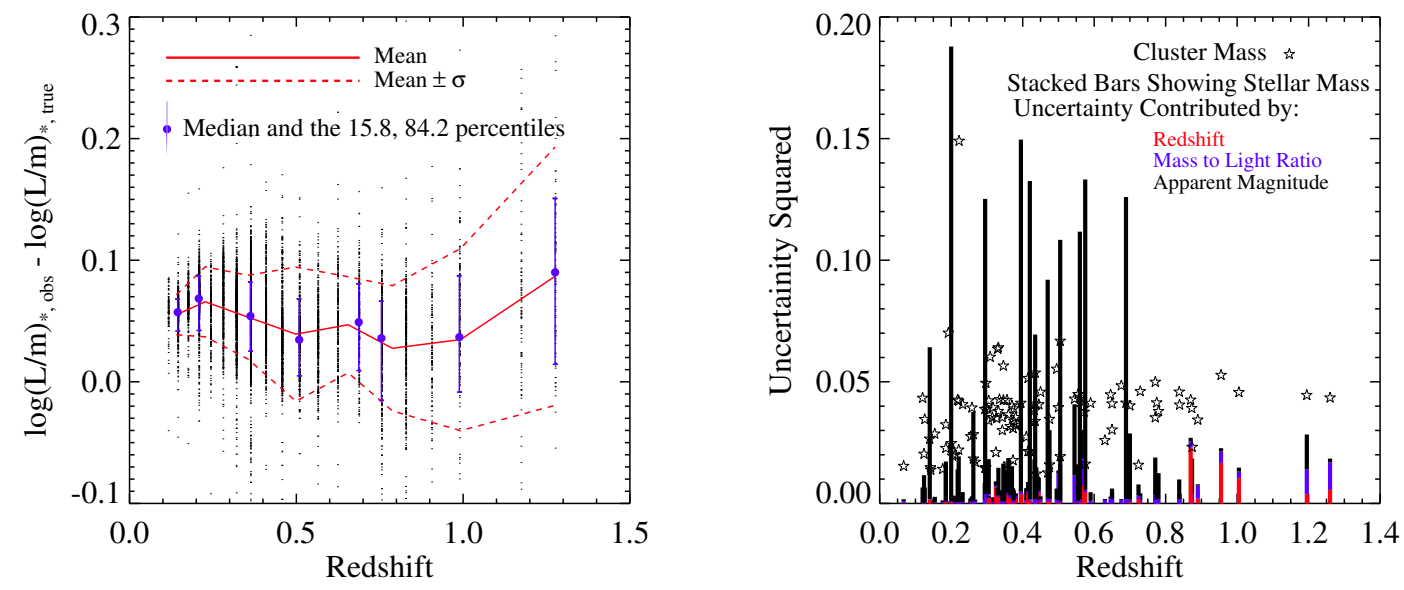

Figure 3.8: (a) We apply our stellar model fitting procedure to the DL07 simulation BCGs using their simulated SDSS $g, r, i, z$ photometry. Because the fitting procedure and the simulated BCG photometry are based on different SSP models and IMFs, there exists a 0.05 dex systematic offset. The derived mass-to-light ratio also has statistical uncertainty of $\sim 0.05$ dex at $z=0$ and $\sim 0.1$ dex at $z=1$. (b) We evaluate the stellar mass uncertainty combining redshift, magnitude and mass-to-light ratio uncertainties. The stellar mass uncertainty is dominated by apparent magnitude uncertainty at $z<0.8$, and redshift uncertainty at $z>0.8$. 
BCG stellar mass. We evaluate the uncertainty and covariance for our BCG sample taking the corresponding values in simulation. To elaborate, for each BCG in our sample, we assume its MLR to have been measured 100 times (each BCG is matched to 100 simulation BCGs in Section 3.6.1), and the error of each measurement is the offset between the derived and true MLR for one simulation BCG. As a result of this set-up, the MLR uncertainty for each BCG contains about 0.05 dex systematic uncertainty and 0.05 to 0.1 dex statistical uncertainty depending on its redshift.

Admittedly, it is more than likely that we are underestimating the BCG MLR uncertainty. In our simulation test, the systematic uncertainty originates from using slightly different SSP models and IMFs (Conroy et al. (2009); Conroy \& Gunn (2010) SSP models and Chabrier (2003) IMF in our procedure VS the Bruzual \& Charlot (2003) SSP models and Kroupa (2001) IMF in DL07). The statistical uncertainty originates from matching $\tau$ star formation history and fixed metallicity to DL07 BCGs. We have not considered uncertainties associated with SSP models, dust distributions and possible IMF variations

Estimating the uncertainties from these so-called "known unknowns" is difficult. Conroy et al. (2009) shows that one may at best recover the MLR of bright red galaxies with 0.15 dex uncertainty at $\mathrm{z}=0$, or $0.3 \mathrm{dex}$ at $\mathrm{z}=2.0$. According to this result, we would have under-estimated BCG MLR uncertainty by $\sim 0.1$ dex. We also experimented with the SSP models from Maraston (2005) and Bruzual \& Charlot (2003), but the derived MLR differences are lower than 0.1 dex.

Since the redshift dependence of our estimation is qualitatively similar to that presented in Conroy et al. (2009), it is un-likely that we are affected with our conclusion about BCG redshift evolution. We therefore do not attempt to include additional uncertainties from the "known unknowns". Eventually, the BCG stellar mass uncertainty is dominated by the uncertainty from magnitude measurement or redshift (See Figure 3.8b), rather than from MLR.

\subsection{Simulation Matching Analysis}

We first inspect the redshift evolution of BCG luminosity and stellar mass through matching our data with a semi-analytical simulation. We compare BCG luminosities and stellar masses to the corresponding values in the simulation, with diagrams analogous to those presented in many previous studies (Collins et al., 2009; Lu et al., 2014; Lidman et al., 2012; Tonini et al., 2012; Lin et al., 2013b; Oliva-Altamirano et al., 2014) that overlay 


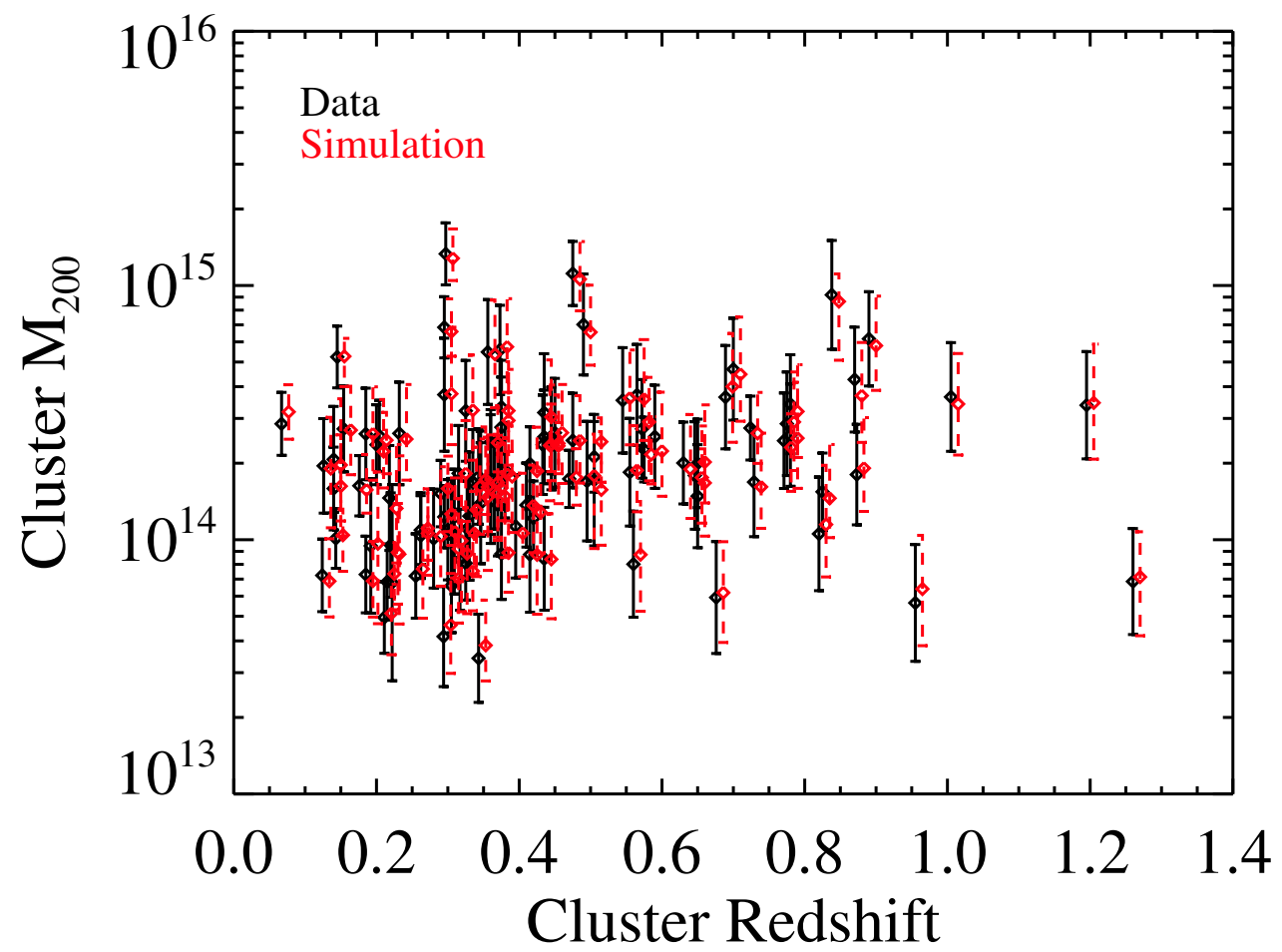

Figure 3.9: Mass and redshift distribution of the cluster sample used for this work and distribution of the simulation clusters drawn from DL07. The black data points show the masses and mass uncertainties of the XCS clusters. The red data points show the median masses and the 0.158 and 0.842 percentiles of the simulation clusters. For clarification, we show the mass distribution of the resampled DL07 clusters at the redshift of the corresponding XCS cluster with a small offset. 

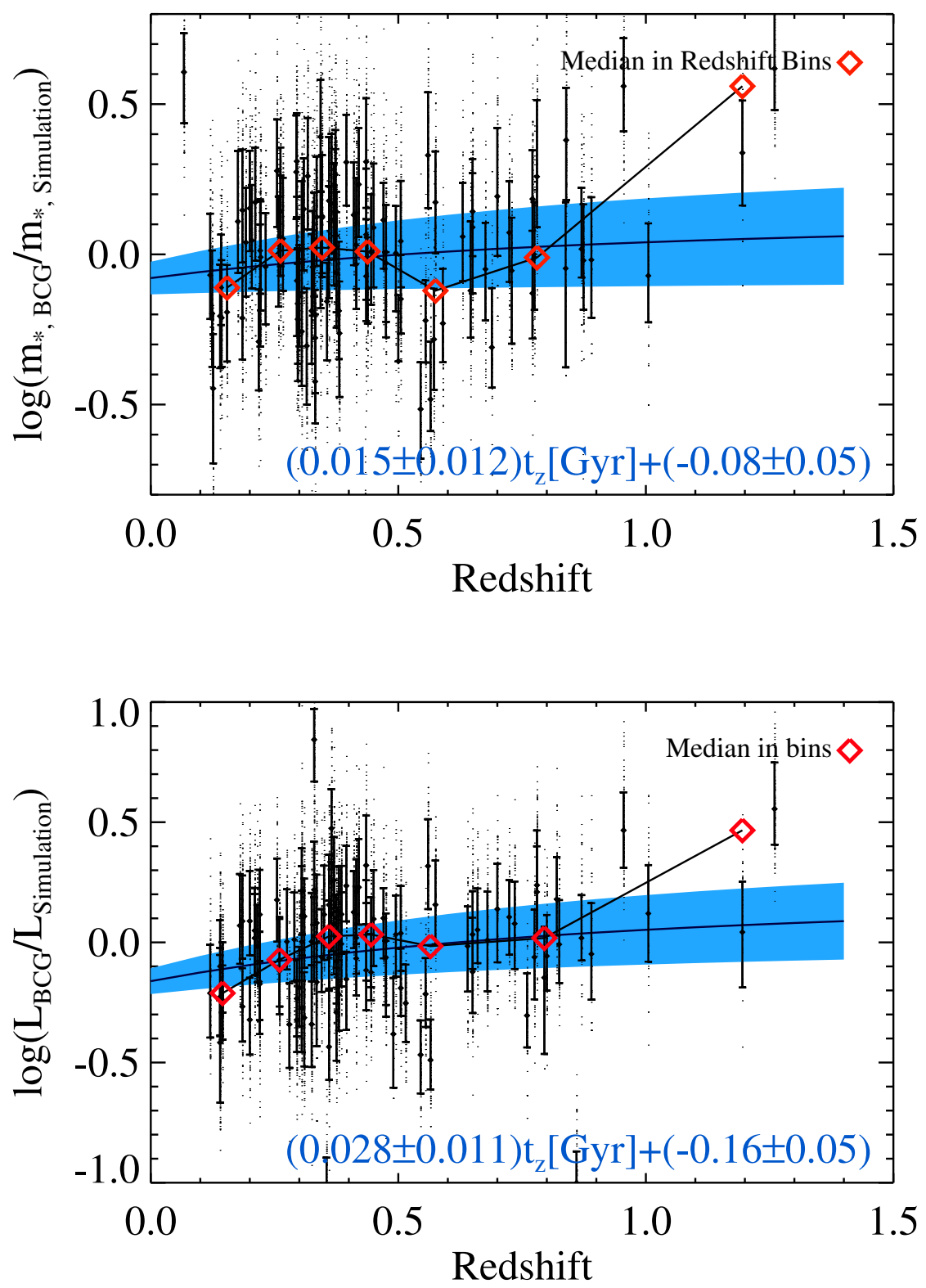

Figure 3.10: Comparison between the observed and simulated BCG properties. We show the median and $0.158,0.842$ percentiles of the differences. The dots are data points outside the range of the uncertainty whiskers, and the red diamonds are moving medians. The blue bands show the fitted linear model with dependence on look-back time, encompassing $1 \sigma$ uncertainties. We notice that the observed BCGs are becoming under-massive/underluminous at decreasing redshift. Note that we have not included stellar mass or luminosity measurement uncertainties in the linear fit. We also use a 95\% confidence interval clipping technique to remove outliers. 
redshift evolutions of the observed and simulated BCG properties. The simulation involved in this comparison is the De Lucia \& Blaizot (2007) semi-analytical (SAM) simulation (referred as DL07 hereafter) based on the Millennium project (Springel et al., 2005; Guo et al., 2013).

\subsubsection{Simulation Sample Selection}

Since BCG luminosity and stellar mass are known to be correlated with cluster mass, the comparison between observation and simulation need to be made between clusters of similar masses. For each BCG in our sample, we compare it to a simulation subsample of 100 BCGs hosted by clusters of similar masses and redshifts. The simulation data are selected with the following procedure.

1. Identify simulation clusters with redshifts closest to that of the XCS cluster. Ideally, we would have identified a cluster sub-sample with their redshift distribution matching the redshift uncertainty of the XCS cluster, but this is not possible since simulations are stored at discrete redshifts.

2. Select from the redshift sub-sample of 100 clusters with their posterior mass distribution (log-normal) matching the mass uncertainty of the XCS cluster. Note that we are not using the cluster mass function as a prior. Application of this prior leads to sampling clusters $\sim 0.1$ dex less massive, but leave the conclusions unchanged.

Note that in the above procedure, we are not considering additional cluster properties beyond $M_{200}$ and redshift. There is emerging evidence that X-ray selected clusters may be biased in terms of cluster concentration distribution (Rasia et al., 2013), but it is un-clear how the bias would affect BCG formation study. We also do not consider the Eddington bias associated with $L_{X}$. The $M_{200}$ of the lowest $L_{X} / T_{X}$ systems are derived with $T_{X}$. Future studies yielding higher precision on BCG growth may wish to take these selection effects into consideration.

In Figure 3.9, we show the redshift and the mass distribution of the XCS clusters together with the re-sampled DL07 simulation clusters. The above procedure produces a simulation sub-sample that well resembles the probability distribution of the XCS sample. 


\subsubsection{Redshift Evolution of the Observed BCGs}

We directly compute the relative luminosity ${ }^{3}$ and stellar mass difference between the observed and simulated BCGs, as shown in Figure 3.10.

We notice that the differences between the observed and simulated BCGs change with redshift. The effect suggests that the observed BCGs do not grow as rapidly as in DL07 a different redshift evolution history in the observation. We fit the differences with a linear dependence on lookback time: if the redshift evolution of the observed BCGs is consistent with that in DL07, the slope of the linear fit shall be 0 . This null hypothesis is not favored.

In Figure 3.10, we show the linear fitting result with blue bands which encompass the $1 \sigma$ uncertainties. The luminosity redshift evolution in the observation is different from the simulation with a $2.5 \sigma$ significance $(0.028 \pm 0.011)$. The significance from the stellar mass comparison is lower at $1.3 \sigma(0.015 \pm 0.012)$, but BCG stellar mass is less certain (recall that it requires a choice for the mass-to-light ratio) and therefore the result is noisier.

The redshift evolution difference shows that the observed BCGs become increasingly under-massive/under-luminous at decreasing redshift compared to DL07 (compare the result to Lidman et al., 2012; Lin et al., 2013b; Oliva-Altamirano et al., 2014). At the lowest redshift bin $(z \sim 0.1)$ in Figure 3.10, the observed BCGs appear to be 0.1 to 0.2 dex ${ }^{4}$ under-massive/under-luminous as a result of a different redshift evolution history.

Arguably, the above statement relies on a fitting function connecting the difference between the observed and simulated BCG properties to redshift. The significance level of this statement depends on the exact form of the fitting function. In Section 3.7 and 3.8, we present stronger evidence on this statement, through modeling the BCG redshift evolution for both observational data and simulation data, testing the model and eventually showing the model constraints being different in the observation and in the simulation.

In addition, we are not considering BCG luminosity and stellar mass uncertainties in this section (they are not included in the linear fitting procedure). We also address this in Section 3.7 and 3.8 .

\footnotetext{
${ }^{3}$ We are comparing the observer frame DES $z$ band luminosity to the observer frame SDSS $z$ band luminosity in DL07. The response curves of the DES $z$ band and the SDSS $z$ band are similar enough, that the magnitude measurements for one object in the two systems shall be close within 0.05 mag. We have tested this statement through cross matching galaxies in the SDSS stripe 82 database and the DES Year 1 coadd database. Although it is possible to transform between DES $z$ band magnitudes and SDSS $z$ band magnitudes, we avoid doing so because the transformation inevitably makes assumption about BCG SEDs.

${ }^{4} x \operatorname{dex}=10^{x}$
} 


\subsubsection{High Redshift BCGs}

At $z>0.9$, we notice that two of the four BCGs in our sample appear to be massive/luminous outliers by $\sim 0.5$ dex, which matches previous findings about massive BCGs at $z>1.0$. In Collins et al. (2009), five $1.2<z<1.5$ BCGs are identified to be $0.5 \sim 0.7$ dex more massive than the DL07 simulation BCGs, and in Liu et al. (2013), a massive $z=1.096 \mathrm{cD}$ type galaxy is discovered in a $5 \operatorname{arcmin}^{2}$ Hubble Deep Field. However, after considering cluster mass uncertainty, and the BCG luminosity and stellar mass uncertainties, we can only detect the over-massive/over-luminous BCG effect with $\sim 1 \sigma$ significance.

\subsection{BCG-Cluster Mass Relation}

To further investigate the growth of BCGs, we turn to modeling a redshift-dependent, stellar-to-halo mass relation. We refer to this relation as the BCG-Cluster mass relation in this chapter. Later, in Section 3.8, we use this relation to model the BCG growth rate from $\mathrm{z}=1.0$ to $\mathrm{z}=0$.

\subsubsection{Modeling the BCG-Cluster Mass Relation}

We model the BCG-Cluster Mass Relation as redshift dependent with the following equation,

$$
\log m_{*}=\log m_{0}+\alpha \log \left(\frac{M_{200, z}}{\mathrm{M}_{\mathrm{piv}}}\right)+\beta \log (1+z) .
$$

This equation adopts a power law dependence on cluster mass (Oliva-Altamirano et al., 2014; Kravtsov et al., 2014; Brough et al., 2008; Moster et al., 2010, 2013) as well as a power law dependence on redshift. We choose $\mathrm{M}_{\text {piv }}$ to be $1.5 \times 10^{14} M_{\odot}$, about the median mass of the XCS clusters. We also assume that there exists an intrinsic scatter, $\epsilon$, between the observed BCG stellar mass and this relation, as $\log m_{*, o b s} \sim \mathcal{N}\left(\log m_{*}, \epsilon^{2}\right)$. Hence, the relation contains four free parameters: $\log m_{0}, \alpha, \beta$ and $\epsilon$.

We perform a Markov Chain Monte Carlo (MCMC) analysis to sample from the following posterior likelihood:

$$
\log \mathcal{L}=-\frac{1}{2} \log |\mathbf{C}|-\frac{1}{2} \mathbf{Y}^{\mathrm{T}} \mathbf{C}^{-\mathbf{1}} \mathbf{Y}+\log p(\mathbf{Q}) .
$$

In this function, $\mathbf{Y}$ is a 106 dimension vector $\left(y_{1}, y_{2} \ldots, y_{106}\right)$, with the $k$ th element being 

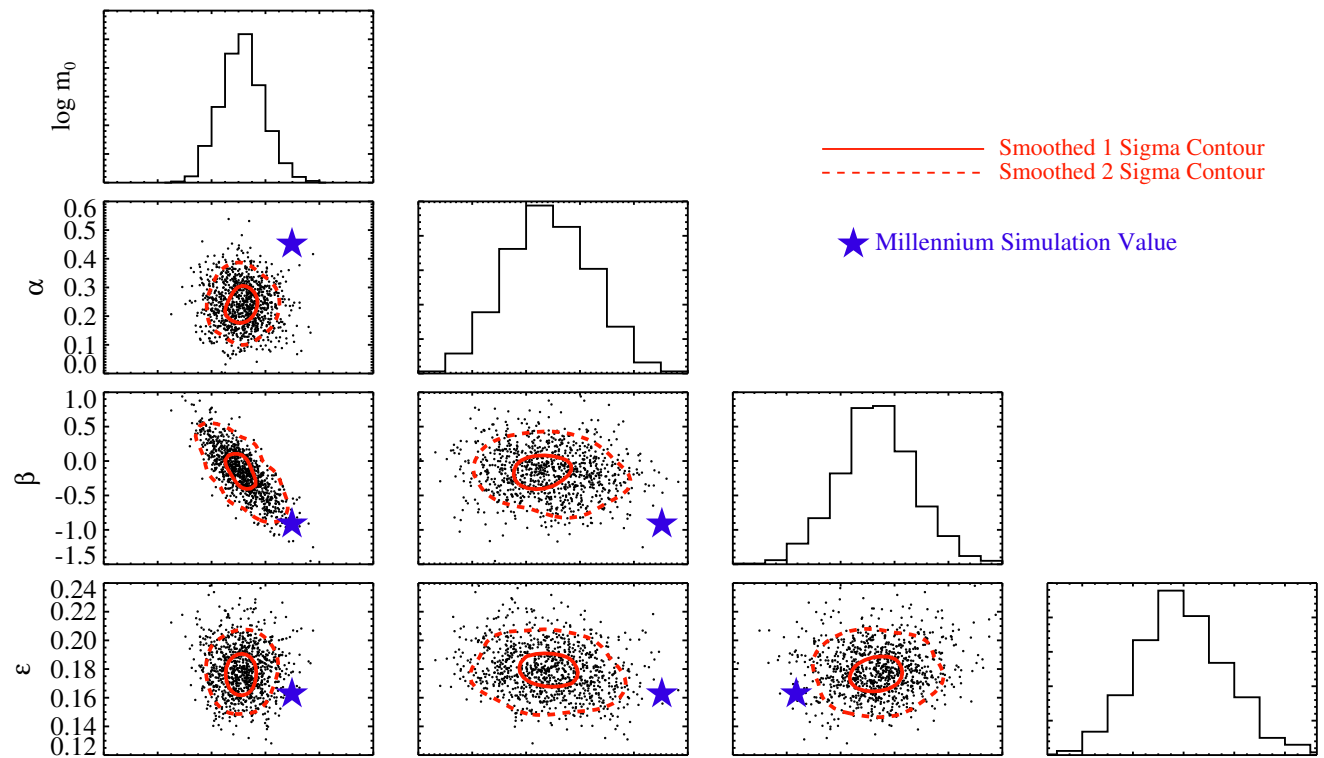

11.011 .211 .411 .611 .812 .0
$\log \mathrm{m}_{0}$

$\alpha^{0.3}$

$\beta$

0.140 .160 .180 .200 .22

Figure 3.11: Posterior distribution of the parameters, $\log m_{0}, \alpha, \beta$ and $\epsilon$, in the BCG-cluster mass relation (Equation 3.1), based on the BCG stellar mass derived in $32 \mathrm{kpc}$ aperture. The histogram in each column shows the marginalized distribution of the corresponding parameter. Other panels show the correlation between two parameters noted on the $\mathrm{x}$ and $\mathrm{y}$ axes. 


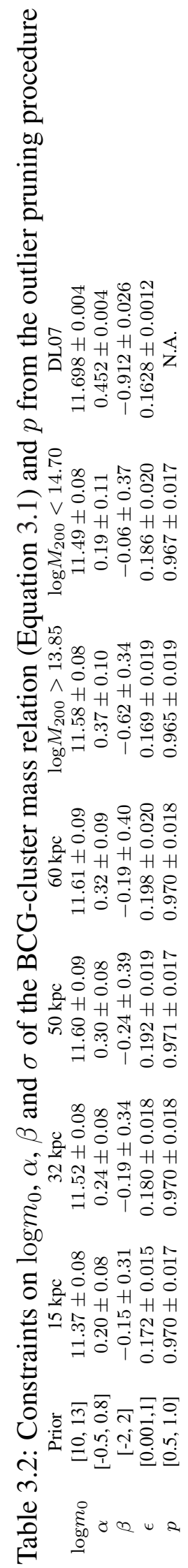


the difference between the modeled and the observed BCG stellar masses, as:

$$
y_{k}=y_{\text {model }, \mathrm{k}}-y_{\mathrm{obs}, \mathrm{k}} \text {. }
$$

The covariance matrix, $\mathrm{C}$, in Equation 3.2 is the combination of the covariance matrices for cluster mass measurements, BCG stellar mass measurements, redshift measurements and the intrinsic scatter. It has the following form:

$$
\mathbf{C}=\operatorname{Cov}\left(\mathbf{m}_{*}\right)+\alpha^{2} \operatorname{Cov}\left(\log \mathbf{M}_{\mathbf{2 0 0}}\right)+\beta^{2} \operatorname{Cov}(\log (1+\mathbf{z}))+\epsilon^{2} \mathbf{I} .
$$

Details about deriving this covariance matrix as well as the likelihood function are provided in the next sub-section.

Additionally, we implement an outlier pruning procedure as we "fit" (or sampling the posterior distribution in Bayesian statistics) for the BCG-cluster mass relation, as described in Hogg et al. (2010). To summarize this procedure, we adopt a set of binary integers $\mathbf{Q}=\left(\mathrm{q}_{1}, \mathrm{q}_{2}, \ldots, \mathrm{q}_{106}\right)$ as flags of outliers. $\mathrm{q}_{k}=0$ indicates an outlier and $y_{k}$ is correspondingly modified as,

$$
y_{k}=\log m_{*, k}-\log m_{\text {outlier }},
$$

where $m_{\text {outlier }}$ is treated as a 5 th free parameter. To penalize data pruning, we assume a Bernoulli prior distribution for $\mathbf{Q}$, characterized by another free parameter $p$ as,

$$
p(\mathbf{Q})=\prod_{k} p^{q_{k}}(1-p)^{1-q_{k}}
$$

Eventually, the parameters to be sampled from Equation 3.2 are $\log m_{0}, \alpha, \beta, \epsilon, \mathbf{Q}$, $p$, and $\log m_{\text {outlier. }}$. More details about deriving the posterior likelihood (Equation 3.2) as well as choosing the covariance matrix can be found in Section 3.7.2. We assume uniform truncated priors for all the free parameters except $\mathrm{Q}$, and the final result appears to be insensitive to this choice. We perform the fitting procedure for both the observed BCGs from the XCS sample and the simulation BCGs sampled from the DL07 simulation (Section 3.6.1).

\subsubsection{Additional Information on Covariance and the Likelihood Func- tion}

This section provides extra information about the likelihood function presented in the Section 3.7. We assume multivariate normal distribution for Y. Combined with a Bernoulli prior distribution for $\mathrm{Q}$, the posterior likelihood becomes: 


$$
\mathcal{L} \propto p(\mathbf{Q}) \times|\mathbf{C}|^{-\frac{1}{2}} \exp \left(-\frac{1}{2} \mathbf{Y}^{T} \mathbf{C}^{-1} \mathbf{Y}\right)
$$

Re-write the likelihood at the log scale and ignore the normalization of the probability distribution, we have:

$$
\log \mathcal{L}=-\frac{1}{2} \log |\mathbf{C}|-\frac{1}{2} \mathbf{Y}^{\mathrm{T}} \mathbf{C}^{-\mathbf{1}} \mathbf{Y}+\log p(\mathbf{Q})
$$

Because

$$
\mathbf{Y}=\mathbf{Y}_{\text {meas }}-\mathbf{Y}_{\text {model }}
$$

The covariance matrix used in the posterior likelihood can be further expanded as :

$$
\begin{aligned}
\mathbf{C} & =\operatorname{Cov}(\mathbf{Y}, \mathbf{Y}) \\
& =\operatorname{Cov}\left(\mathbf{Y}_{\text {model }}-\mathbf{Y}_{\text {obs }}, \mathbf{Y}_{\text {model }}-\mathbf{Y}_{\text {obs }}\right) \\
& =\operatorname{Cov}\left(\mathbf{Y}_{\text {obs }}, \mathbf{Y}_{\text {obs }}\right)+\operatorname{Cov}\left(\mathbf{Y}_{\text {model }}, \mathbf{Y}_{\text {model }}\right) \\
& =\operatorname{Cov}\left(\mathbf{m}_{*, \text { obs }}, \mathbf{m}_{*, \text { obs }}\right)+\alpha^{2} \operatorname{Cov}\left(\log \mathbf{M}_{\mathbf{2 0 0}}, \log \mathbf{M}_{\mathbf{2 0 0}}\right)+\beta^{2} \operatorname{Cov}(\log (1+\mathbf{z}), \log (1+\mathbf{z}))+\sigma^{2} \mathbf{I} .
\end{aligned}
$$

We have simplified this expression as Equation 3.4 in Section 3.7.

If the covariance matrices for cluster redshift, cluster $M_{200}$ and BCG stellar mass are all diagonal, our posterior likelihood function - Equation 3.2 - would have the same form as that derived in Hogg et al. (2010). In our case, the covariance matrices for $M_{200}$ and BCG stellar masses are not diagonal because of systematic uncertainties (See details in Sections 3.3 to 3.5$)$.

\subsubsection{Constraints on the BCG-Cluster Mass Relation}

In Figure 3.11, we plot the posterior distribution of $\log m_{0}, \alpha, \beta, \epsilon$ in Equation 3.1. We also list their marginalized means and standard deviations in Table 3.2.

The constraint we derive on $\alpha$ agrees well with the reported values from the literature (Oliva-Altamirano et al., 2014; Kravtsov et al., 2014; Brough et al., 2008). We also notice that $\alpha$ increases with bigger BCG apertures, indicating stronger correlation with cluster mass in the BCG outskirts (also see Stott et al., 2012). This effect seems to be justifiable, considering an inside-out growth scenario for BCGs (van Dokkum et al., 2010; Patel et al., 2013; Bai et al., 2014). Further analysis with large apertures is limited by the increasing amount of background noise at BCG outskirt, but a larger BCG sample may help quantifying the effect. This effect also illustrates the importance of understanding BCG photometry 
measurement when deriving BCG-cluster mass relations.

Our estimation of $\log m_{0}$, the normalization of Equation 3.1, appears to be lower than the corresponding value in DL07 by $0.1-0.2$. As $\log m_{0}$ is mainly constrained by low redshift BCGs, this result is completely consistent with BCGs being under-massive at low redshift as discussed in Section 3.6.2.

Our estimation of $\beta$, the index of the redshift component in Equation 3.1, also disagrees with the corresponding value in DL07. The constraint on $\beta$ derived from the whole cluster sample is different from the simulation value at a significance level of $2.3 \sigma$. The constraint from our data is closer to 0 , suggesting less change of BCG stellar mass with redshift. Note that a further, quantitative conclusion should not be drawn. Although $\beta$ is the dominant parameter that describes BCG redshift evolution in Equation 3.1, it is not the only one. The mass term in Equation 3.1 also contains information about BCG redshift evolution as cluster $M_{200}$ evolves with time. A quantitative analysis of BCG redshift evolution is presented in Section 3.8.

Our constraint on $\beta$ is highly co-variant with $\log m_{*}$ (recall the bi-variate normal distribution), but the co-variance shall not be interpreted as "degeneracy": a reasonable $m_{*}$ sampled from its marginalized posterior distribution does not make $\beta$ consistent with the simulation. We also notice that different conventions for BCG magnitude measurement can bias the constraint on $\beta$. For example, using the Kron magnitude from the popular SExtractor software (Bertin \& Arnouts, 1996), which tends to under-estimate BCG Kron Radius and therefore BCG total magnitude (See discussion in Section 3.4.2. This effect happens frequently for our intermediate redshift BCGs), shifts $\beta$ downward by $\sim 1 \sigma$.

We detect hints that the constraints on $\alpha$ and $\beta$ may depend on cluster mass (see Table 3.2). For clusters with $\log M_{200}$ above 13.85, we notice stronger correlation between BCG and cluster masses (larger $\alpha$, compare it to Chiu et al., 2014; van der Burg et al., 2014) and steeper redshift evolution (smaller $\beta$ ) at $\sim 1.0 \sigma$ significance level. However, BCGs in low mass clusters $\left(\log M_{200}<13.85\right)$ are possibly over-massive compared to our simulation calibrated BCG-cluster mass relation. Evaluating the masses of low mass clusters and groups through their X-ray observables needs to be handled with care. In this work, we use lensing calibrated $M-T$ relation of galaxy groups and clusters to derive $M_{200}$ for most low mass clusters (see Figure 3.4 and Section 3.3). Arguably, the accuracy of X-ray inferred masses of low mass clusters is less well characterized than the higher mass end. Thus, in our growth rate determinations we show the difference after excluding the lowest mass systems $\left(\log M_{200}<13.85\right.$, about $10 \%$ of the sample). For consistency, we also examine the effect of excluding the highest mass systems $\left(\log M_{200}>14.7\right.$, about $10 \%$ of the sample). 


\subsection{BCG Stellar Mass Growth since $z \sim 1.0$}

In this section, we compute the BCG stellar mass growth rate since redshift 1.0. We derive the growth rate conveniently using the redshift-dependent BCG-cluster mass relation from the previous section. Doing so, we are assuming that a redshift-dependent BCG-cluster mass relation not only describes the relation between BCG stellar mass and cluster mass at various redshifts, but also describes how BCG stellar mass evolves with time. There is no new measurement made with observational data in this section. The redshift-dependent BCG-cluster mass relation derived in the previous section is the only input from observational data. Our method, however, do need new input from simulation data, which is the mass evolution history of clusters.

In this section, we compute the stellar mass growth rate for the BCGs hosted by clusters of $\log M_{200}=13.8$ at $z=1.0$. The choice is made as the XCS sample well represents these clusters and their low redshift descendants (see Figure 3.12 for the mass evolution history of clusters with $\log M_{200}=13.8$ at $z=1.0$ ). The method is also applied to clusters of different masses, but we do not notice significant change of the conclusions.

\subsubsection{Method and Test}

We need to know how the cluster mass evolves with redshift in our method. To acquire this information, we select a sample of halos with $z \sim 1.0, \log M_{200} \sim 13.8$ from the Millennium simulation, and extract their evolution history by identifying descendants of these halos all the way to $z=0$ (using the descendantid keyword). We then compute the mean $M_{200}$ evolution of these halos, shown in Figure 3.12.

The second step is to use the BCG-cluster mass relation to derive the average stellar mass of the BCGs hosted by these halos at different redshifts. From Equation 3.1, the average $\mathrm{BCG}$ stellar mass relative to some normalization epoch, $z_{0}$, can be expressed as:

$$
\log \frac{m_{*, z}}{m_{*, z_{0}}}=\alpha \log \frac{M_{200, z}}{M_{200, z_{0}}}+\beta \log \frac{1+z}{1+z_{0}} .
$$

We take $\log \frac{m_{*, z}}{m_{*, z_{0}}}$ from the above equation as describing the average BCG stellar mass

growth. The $\frac{M_{200, z}}{M_{200, z_{0}}}$ component in the equation is the average cluster mass growth extracted from the simulation.

The result of applying Equation 3.11 to the average $M_{200}$ growth with the simulation data is also shown in Figure 3.12. We estimate the uncertainties on the BCG stellar mass growth rate through sampling the joint constraint on $\alpha$ and $\beta$. We do not consider the uncertainties of cluster mass evolution as it is marginal and is cosmology dependent. 

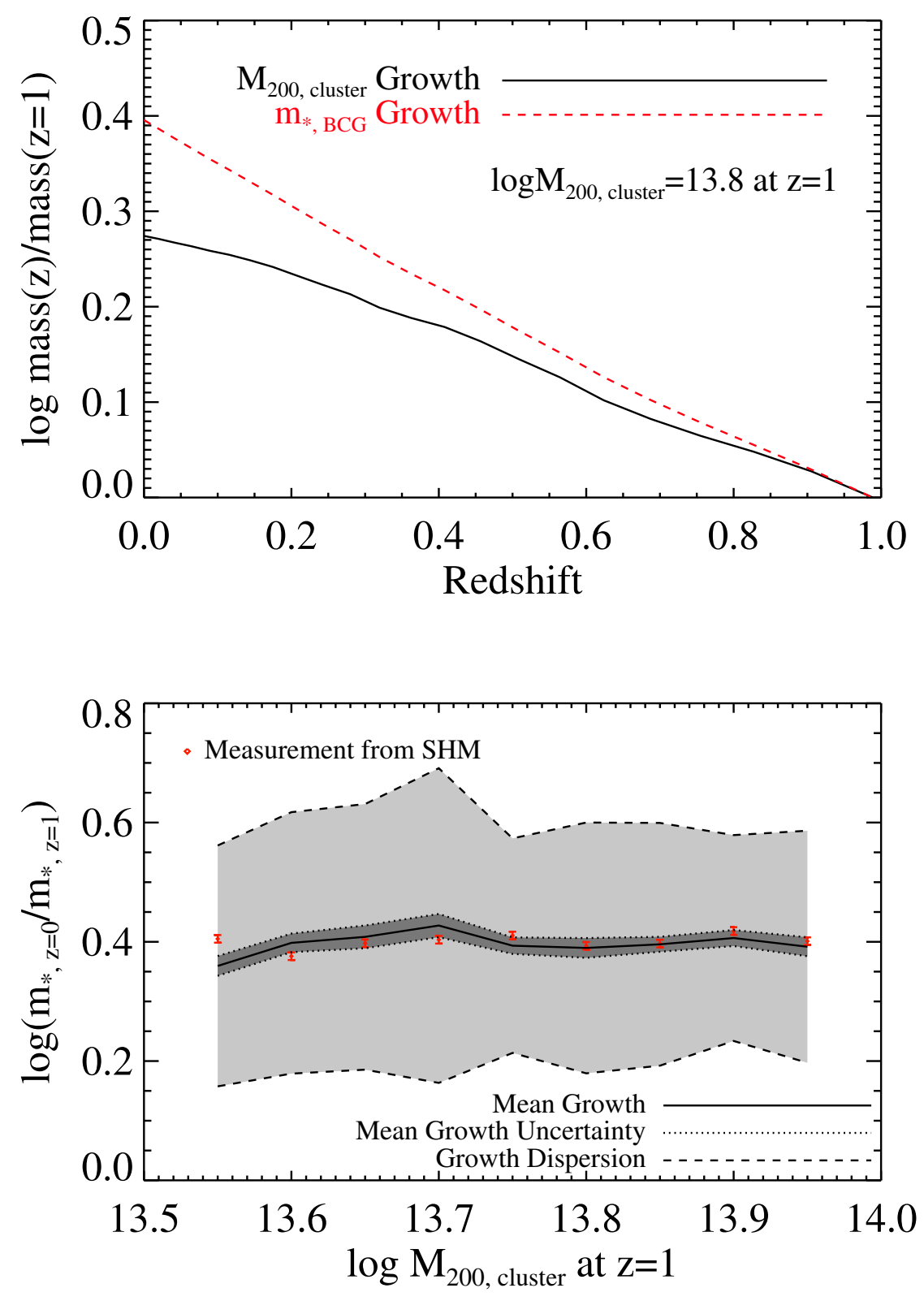

Figure 3.12: To derive BCG stellar mass growth rate from BCG-cluster mass relations, we will need to derive cluster mass growth history from simulations. In the top panel, we show the halo mass evolution history of $\sim 200$ halos with $\log M_{200}=13.8$ at $z=1.0$. We utilize Equation 3.11 (we are using the BCG-cluster mass relation in DL07) to derive the BCG stellar mass growth rate shown by the red dashed line. In the bottom panel, we show the test result for this method (see Section 3.8.1 for details). Overall, our approach well reproduces the average growth rate within $1 \sigma$ for simulation BCGs. 


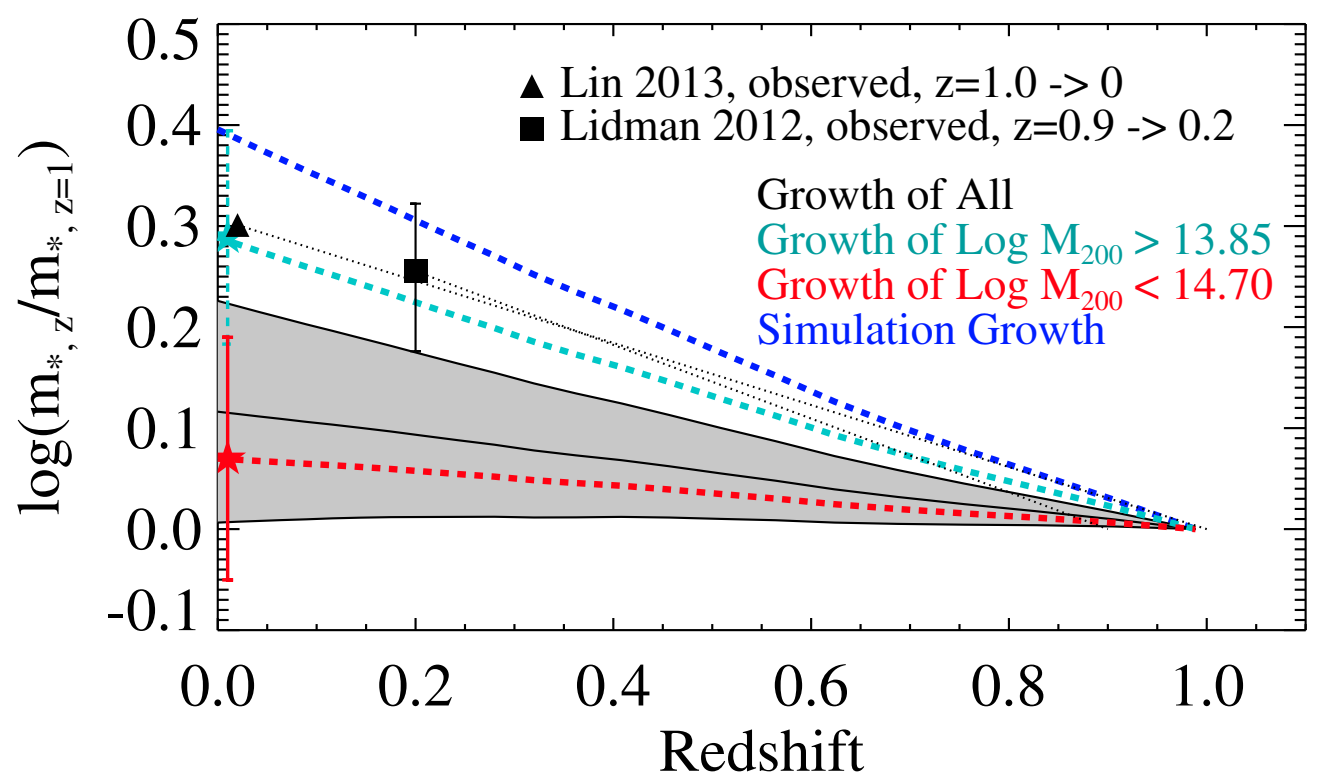

Figure 3.13: This plot shows our BCG stellar mass growth estimation with the full sample and with two mass-limited sub-samples. We also show the measurements in Lidman et al. 2012 and Lin et al. 2013 and the BCG growth rate in the DL07 simulation. Our estimation is consistent with previous measurements, but slower than DL07 by $\sim 2.5 \sigma$. The uncertainty from DL07 is extremely small as the simulation is well sampled.

We test our method by applying it to the DL07 simulation BCGs. We first derive the BCG-cluster mass relation in DL07 using the procedure from Section 3.7.1 for the sample drawn from Section 3.6.1. We compare the computed BCG growth rate to the values obtained through directly tracking cluster descendants. The latter is acquired through recording the central galaxy stellar mass of the halo descendants since redshift 1.0. We consider the result from this second approach as the true growth of simulation BCGs.

In the bottom panel of Figure 3.12, we show the BCG growth rate derived with Equation 3.1, and the true growth rate encompassed by uncertainty from bootstrapping. Overall, for low mass clusters, our approach reproduces the average BCG growth rate from $z=1.0$ to $z=0$ within $1 \sigma$. Bias associated with this method (like progenitor bias, see: Shankar et al., 2015), if there is any, appears to be negligible.

\subsubsection{Growth Rate from $z=1.0$ to $z=0$}

We compute the BCG stellar mass growth rate using Equation 3.11 and compare it to the simulation value obtained with the same method. We discuss the observational result based on BCG $32 \mathrm{kpc}$ aperture stellar masses in this work - the result derived with other apertures 
look similar. From $z=1.0$ to $z=0$, we estimate the BCG growth rate to be $0.13 \pm 0.11$ dex, comparing to $0.40 \pm 0.05$ dex in simulation (uncertainty estimated for the BCG sample in Section 3.6.1), as shown in Figure 3.13. This result is in agreement with our conclusion from the simulation matching analysis (Section 3.6.2), and also in agreement with previous studies (Lidman et al., 2012; Lin et al., 2013b). Even after considering all the uncertainties, biases and covariances associated with BCG luminosity and stellar mass measurements, we still confirm that the observed BCG growth is slower than the prediction from DL07 at a significance level of $\sim 2.5 \sigma$.

Like our constraint on the BCG-cluster mass relation, our result here shifts by $\sim 1 \sigma$ $(0.29 \pm 0.11 \mathrm{dex})$ when we exclude the lowest mass systems $\left(\log M_{200}<13.85\right.$, about $10 \%$ of the sample). Note that the shift may be caused by inaccuracy of X-ray cluster mass scaling relations at the low mass end (see discussion in Section 3.7.3). For consistency, we also show the result $(0.07 \pm 0.12 \mathrm{dex})$ after excluding the highest mass systems $\left(\log M_{200}>\right.$ 14.7 , about $10 \%$ of the sample). Our result is also susceptible to improper BCG magnitude measurements. Using the Kron magnitude from SExtractor, the result will be biased toward more rapid BCG growth by $\sim 1 \sigma$. We also considered applying our method with stellarto-halo mass relations from literature, but as many previous studies are based on magnitude conventions with various problems for BCGs (see discussion in Appendix 3.4), we opt for not using them in this work.

\subsubsection{Role of ICL Production}

In this chapter, we have shown from two different perspectives that the BCG stellar mass growth rate in clusters with $\log M_{200}=13.8$ at $z=1.0$ is slower than the prediction naively expected in a hierarchical formation scenario (De Lucia \& Blaizot, 2007). This effect is not that surprising with a second thought on the processes that contribute to (or counter-act) BCG formation.

A hierarchical structure formation scenario predicts that galaxy mergers add stars to BCGs. The BCG stellar build-up can be further augmented by in situ star formation, but a reduction in stellar mass is possible from mergers that eject stars into the intracluster space. The competition between these mechanisms remains a subject to large modeling uncertainties in simulations. If we assume that BCGs experience the rapid build-up events (mostly merging events) as prescribed in the DL07 simulation, there must be a mechanism that offsets BCG growth to mimic slower evolution we observe in this work.

In Figure 3.14, we experiment with incorporating extra stellar mass gain or loss into the DL07 simulation. Stellar mass gain tends to steepen BCG growth over time, while stellar 


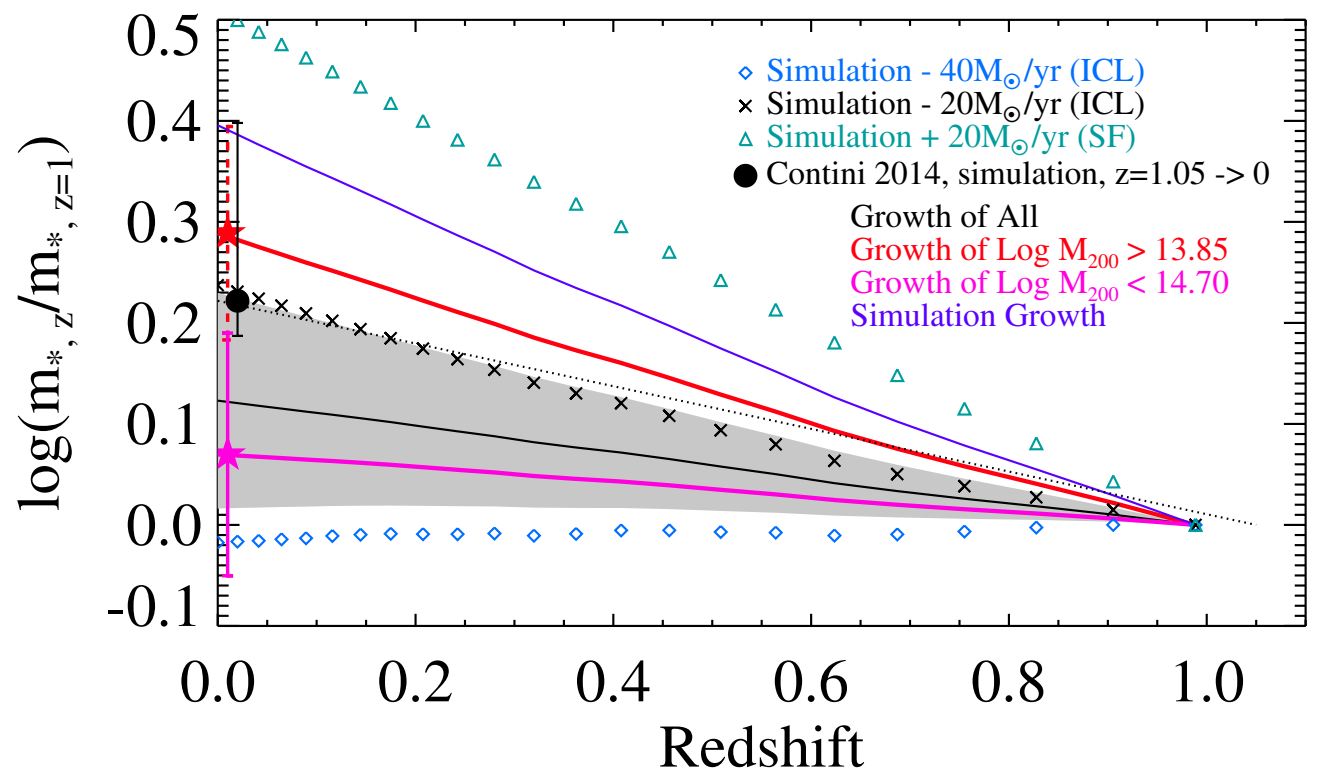

Figure 3.14: We show BCG growth rates from toy models adding more rapid ICL production or more dramatic BCG star formation to the DL07 simulation. Including extra constant ICL production at $20-40 M_{\odot} / \mathrm{yr}$ well reproduces our observed growth rate. We also show the predicted BCG growth rate from Contini et al. 2014, which has updated the DL07 simulation with more realistic ICL production. The BCG growth rate in Contini et al. 2014 agrees well with our measurement. 
mass loss tends to slow down BCG build-up and flatten the BCG growth curve. In order to explain the observed BCG growth rate in our data, the BCGs in DL07 would need to go through extra stellar mass loss at $20-40 M_{\odot} /$ yr, ending up with 2.0-3.5 $\times 10^{11} M_{\odot}$ at $z=0$, which agrees with our data.

Such stellar content stripping from mergers would produce intra-cluster light (ICL). Our result indicates that ICL accumulates at 20-40 $M_{\odot} /$ yr after $z=1.0$, totaling $(1.5-$ $3) \times 10^{11} M_{\odot}$ ICL in the present epoch. This amount corresponds to about $30 \%-60 \%$ the total of BCG and ICL stellar masses, consistent with the observed ICL fraction in low and medium redshift clusters $(z<0.5$ : Zibetti et al., 2005; Krick et al., 2006; Krick \& Bernstein, 2007; Gonzalez et al., 2007; Toledo et al., 2011; Montes \& Trujillo, 2014; Giallongo et al., 2014; Presotto et al., 2014).

In fact, ICL production has already been suggested as an explanation to the seemingly mild evolution of massive galaxies (Monaco et al., 2006; Conroy et al., 2007; Burke et al., 2012; Behroozi et al., 2013; Oliva-Altamirano et al., 2014). Although not completely settled, recent studies indicate that ICL possibly forms late, mostly after $z=1.0$ (Contini et al., 2014; Conroy et al., 2007). Specifically, the Contini et al. (2014) study updates the DL07 simulation with more realistic ICL production processes, and predicts slower BCG growth rate (Figure 3.14), in excellent agreement with our measurement. Hence, the slow BCG stellar mass growth since $z=1.0$ observed throughout this work is completely justifiable if ICL forms late after $z=1.0$.

Admittedly, the DL07 simulation also includes stellar stripping that would produce ICL. Unfortunately the amount of ICL from this simulation is not retrievable, and we are not able to analyze if it meets our expectation. The Guo et al. (2011) SAM simulation has explicitly included ICL production and predicts very similar BCG growth with DL07, but much of the ICL is already in place before $z=1.0$, which is not favored in our interpretation.

\subsection{Summary and Discussion}

Using new photometric data from DES and a new X-ray selected cluster and group sample from the XCS, we investigate the redshift evolution of BCG stellar mass since $z=1.2$. We derive constraints on the BCG-cluster mass relation, and compute the BCG stellar mass growth rate for our sample. From two different perspectives, we demonstrate that the BCG stellar mass growth since $z=1.0$ is slower than the expectation from a semi-analytical simulation implementing a simple hierarchical BCG formation scenario. The discrepancy is detected with a significance level as high as $2.5 \sigma$. We find this slow growth rate after $z=1.0$ to be compatible with the late formation of ICL . 
We have carefully considered various uncertainties related to studying BCG growth in this work, including the uncertainties of BCG stellar mass measurements, cluster/BCG redshift measurements and cluster mass measurements. We explicitly consider these uncertainties through likelihood analysis, and expect this analysis to help clarify ongoing discussions about how statistical and systematic uncertainties affecting BCG growth measurements.

We also adopt a simple but novel method to compute BCG stellar mass growth rate. Despite considerable attention paid to this topic in the literature, BCG stellar mass growth has been studied with various techniques inconsistent with each other. Ideally, one would like to evaluate BCG stellar mass growth by comparing the BCG masses within the same cluster at high and low redshifts, as we did for method testing in Section 3.8.1. This is not possible with observations. However, Lin et al. (2013b) have adopted the idea through constructing a cluster sample that resembles the average halo evolution history. In observational studies, the more common approach is to compare the BCG masses of a high redshift cluster sample and a low redshift cluster sample, while adjusting the cluster mass binning at different redshifts to account for cluster mass evolution (Collins et al., 2009; Lidman et al., 2012; Bai et al., 2014). The results from these observational studies are widely compared to De Lucia \& Blaizot (2007), which computes the BCG stellar mass growth rate through a "fixed space density" method, i.e, selecting the 125 most massive clusters at $z \sim 1.0$ and $z \sim 0$ respectively to compare their BCG masses. Compared to these previous studies, our method allows consistent comparison to simulation for clusters of specific masses and redshifts. Our test in Section 3.8.1 shows that the approach suffers from only negligible bias for the required precision.

Finally, the analyses presented here are based on DES SV data, a data set corresponding to only $5 \%$ of the nominal DES footprint. With spectroscopic and X-ray follow-up, Miller et al. (in prep) show that the final DES/XCS sample should be about 10 times larger than this data set. Comparing the constraints on the BCG-cluster mass relation derived with 1000 simulation clusters rather than 100 of them, we conclude that we expect $\sim 3$ times improvement in the measurement uncertainty of BCG growth. At this level of statistical power, it will be critically important to thoroughly understand the uncertainties associated with various observables. This project presents the first steps toward such an analysis. 


\section{CHAPTER 4}

\section{Evolution of Cluster Red Sequence Galaxies}

\subsection{Topic Introduction}

Galaxy clusters are special for both cosmology and astrophysics studies. As the structures that correspond to the massive end of halo mass function, they are sensitive probes of the $\Lambda$ CDM cosmology model (see reviews in Allen et al., 2011; Weinberg et al., 2013). As the most massive virialized structures in the universe, they provide the sites for studying astrophysics processes in dense environments.

Galaxy clusters are known to harbor red sequence galaxies, named as these galaxies rest on a tight relation in the color-magnitude diagram (Bower et al., 1992b). This phenomenon has been employed in finding clusters from optical data (e.g., Gladders \& Yee, 2000; Miller et al., 2005; Koester et al., 2007b; Rykoff et al., 2014) and developing cluster mass proxies (e.g., Rykoff et al., 2012), which in turn require a good understanding of these galaxies. Red sequence galaxies also attract attention from astrophysics studies as they exhibit little star formation activity. Their formation and evolution provide clues as to how galaxies have been transformed in the cluster environment.

It is relatively well-established that massive red sequence galaxies form at an early epoch (e.g., Mullis et al., 2005; Stanford et al., 2005; Mei et al., 2006; Eisenhardt et al., 2008; Kurk et al., 2009; Hilton et al., 2009; Papovich et al., 2010; Gobat et al., 2011; Jaffé et al., 2011; Grützbauch et al., 2012; Tanaka et al., 2013), but the formation of faint red sequence galaxies remain perplexing. The latter can be examined through inspecting the luminosity distribution of cluster galaxies, either with the dwarf-to-giant ratio approach (De Lucia et al., 2007), or as adopted in this study, with a luminosity function (LF) analysis. Results from these analyses are controversial to date, which problem has been extensively reviewed in literature (e.g., Crawford et al., 2009; Boselli \& Gavazzi, 2014; Wen \& Han, 2015).

To summarize, a few studies have reported a deficit of faint red sequence galaxies with 
increasing redshift (De Lucia et al., 2007; Stott et al., 2007; Gilbank et al., 2008; Rudnick et al., 2009; de Filippis et al., 2011; Martinet et al., 2015), indicating slower formation of faint red sequence galaxies comparing to the bright (and massive) ones. Yet, many others observe little evolution in red sequence luminosity distribution up to redshift 1.5 (Andreon, 2008; Crawford et al., 2009; De Propris et al., 2013), suggesting an early formation for both faint and bright red sequence galaxies. Differences in these results are hard to interpret given the different methods (see the discussion in Crawford et al., 2009), sample selections and possible dependence on cluster mass (Gilbank et al., 2008; Hansen et al., 2009; Lan et al., 2015), dynamical states (Wen \& Han, 2015; De Propris et al., 2013), and whether or not the clusters are fossils (Zarattini et al., 2015). Carrying out more detailed analysis using significantly larger samples especially in the 0.5 to 1.0 redshift range may help resolving these differences.

The luminosity distribution of cluster galaxies has also been modeled to connect galaxies with the underlying dark matter distribution. The luminosity function of galaxies in a halo/cluster of fixed mass, entitled the conditional luminosity function (CLF) in recent literature (Yang et al., 2003), statistically models how galaxies occupy dark matter halos. Modeling the Halo Occupation Distribution (HOD, Peacock \& Smith, 2000) provides another popular yet closely-related approach. Given a dark matter halo distribution, these models (HOD \& CLF) can be linked with several galaxy distribution and evolution properties (e.g., Cooray, 2006; Zheng et al., 2007; van den Bosch et al., 2007; Zehavi et al., 2011; Leauthaud et al., 2012), including galaxy correlation functions (e.g., Jing et al., 1998; Peacock \& Smith, 2000; Seljak, 2000), galaxy luminosity/stellar mass functions (e.g., Yang et al., 2009), global star formation rate (e.g., Behroozi et al., 2013) and galaxy-galaxy lensing signals (e.g., Mandelbaum et al., 2006).

The Sloan Digital Sky Survey has enabled detailed analysis of cluster LF (or CLF) with the identification of tens of thousands of clusters to redshift 0.5 (Yang et al., 2008; Hansen et al., 2009). Above redshift 0.5, studies are still limited to small samples containing a handful of clusters or groups (Andreon, 2008; Rudnick et al., 2009; Crawford et al., 2009; De Propris et al., 2013; Martinet et al., 2015). However, recent development of wide field surveys that are more sensitive than the SDSS, provides an opportunity to reinvigorate our knowledge in this regime.

In this chapter, we constrain the (conditional) red sequence luminosity function (RSLF) with an X-ray selected sample discovered in the DES science verification data. The sample contains clusters and groups in the mass range of $\left[3 \times 10^{13} M_{\odot}, 2 \times 10^{15} M_{\odot}\right]$, and the redshift range of $[0.1,1.3]$. Similar analyses can also be applied to SZ-selected clusters (e.g., clusters discovered from the South Pole telescope survey: Staniszewski et al., 2009; 
Reichardt et al., 2013; Bleem et al., 2015) and clusters selected from optical data. Our work with X-ray selected clusters benefits from the well-understood selection of the sample and the convenience of having X-ray temperature and luminosity as cluster mass proxies. Using this sample also avoids possible selection effect on red sequence galaxies.

This chapter focuses on constraining the luminosity function of red sequence galaxies. The luminosity function of blue galaxies generally deviates from that of the red, but constraining it seems to require a larger sample because of the blue galaxy low over-density. In the rest of the chapter, we describe and characterize our data sets in Section 4.2, evaluate the photometric data completeness limit in Section 4.4 and describe our methods in Section 4.5. We present our results in Section 4.6, and discuss the implications in Section 4.7. A summary of this chapter is provided in Section 4.8 .

\subsection{Data}

\subsubsection{Dark Energy Survey Science Verification Data}

We use the DES Science Verification (DES-SV) data taken in late 2012 and early 2013. The DES collaboration collected this data set with the newly mounted Dark Energy Camera (DECam, Flaugher et al., 2015b) for science verification purposes before the main survey began (see DES Year 1 operations in Diehl et al., 2014). In total, this data set covers $400 \mathrm{deg}^{2}$ of the sky. For about $200 \mathrm{deg}^{2}$ of it, data are available in all of the $g, r, i, z$ and $Y$ bands, and the total exposure time in each band fulfills the DES full depth requirement (23 to $24 \mathrm{mag}$ in $i$ and 22 to $23 \mathrm{mag}$ in $z$, see more details in Sánchez et al., 2014). A pilot supernovae survey (see Papadopoulos et al., 2015, for an overview) of $30 \mathrm{deg}^{2}$ sky in $g, r$, $i, z$ was conducted at the same time, reaching deeper depth after image coaddition $(\sim 25$ mag in $i$ and $\sim 24$ mag in $z$ ).

The Science Verification data are processed with the official DES data reduction pipeline (Mohr et al., 2012). In this pipeline, single exposure images are assessed, detrended, calibrated and coadded. The coadded images are then fed to the SExtractor software (Bertin \& Arnouts, 1996; Bertin, 2011) for object detection and photometry measurement.

\subsubsection{The XCS-SV cluster sample}

The XCS-SV cluster sample is a product from the XMM Cluster Survey (Lloyd-Davies et al., 2011; Mehrtens et al., 2012; Viana et al., 2013), which searches for galaxy cluster 


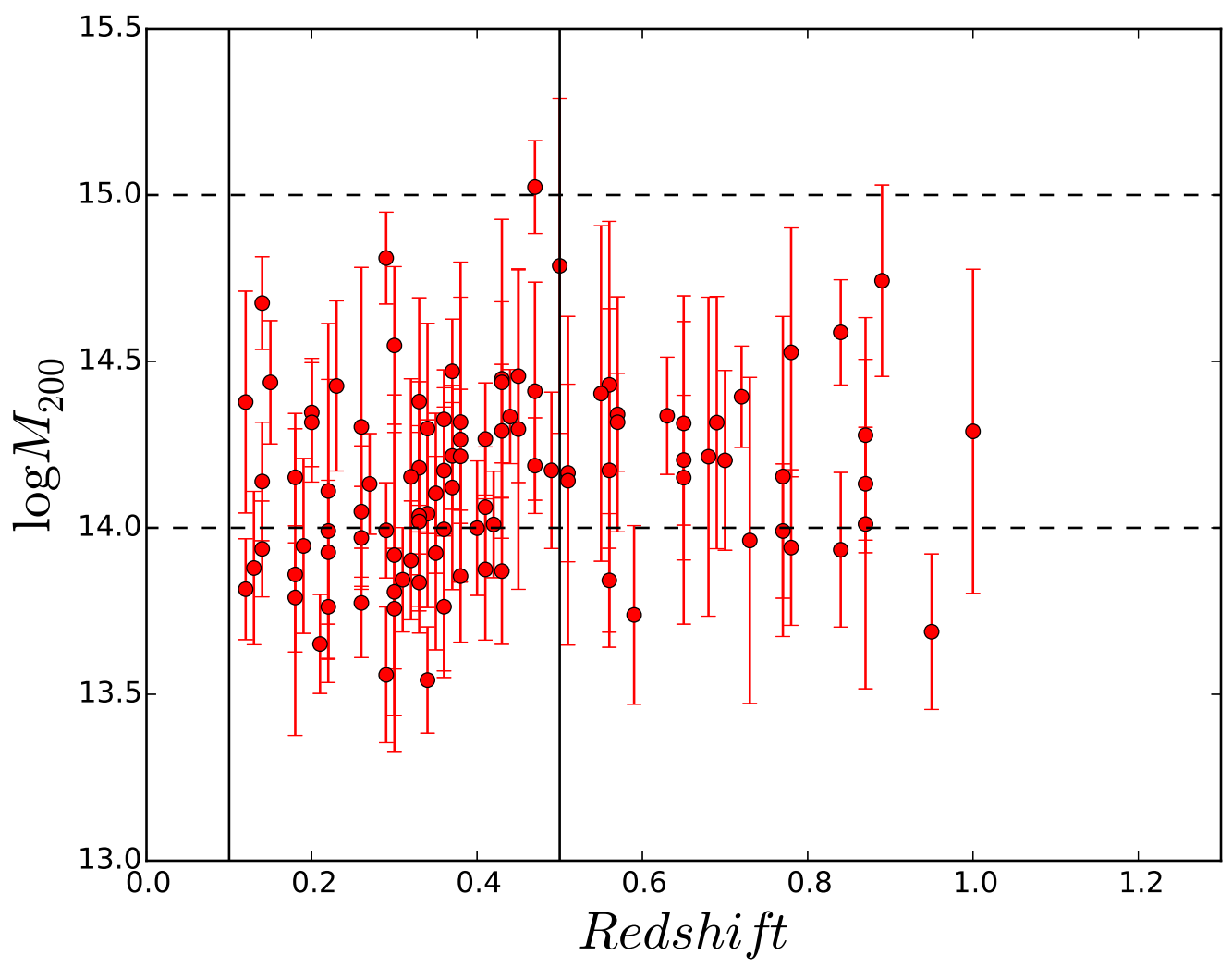

Figure 4.1: The redshifts, masses, and mass uncertainties of the XCS-SV clusters. Vertical solid lines and Horizontal dashed lines show the ranges of the subsamples used to constrain LF mass dependence and redshift evolution respectively. 
candidates in XMM-Newton archival data. The XCS-SV sample refers to the confirmed clusters with the DES-SV optical data (Miller et al. in prep., henceforce referenced as M15). This X-ray selected sample contains galaxy groups, low mass clusters and clusters as massive as $10^{15} \mathrm{M}_{\odot}$ up to redshift 1.3. In Zhang et al. (2015c, henceforce referenced as Z15), we review the selection and confirmation of the sample, as well as the cluster photometric redshift measurement.

Since this study evaluates cluster galaxy luminosity function with $z$ band photometry, we eliminate clusters above redshift 1.05 so that the rest frame $4000 \AA$ break of the RS galaxy spectrum do not lie in DES $z$-band (sensitive to beyond $\sim 8500 \AA$ ). We further eliminate one cluster below redshift 0.1 that is more susceptible to X-ray contaminations and also the clusters that are not in ideal photometry regions (Section 4.2.3). This chapter works with 102 clusters in total. In Figure 4.1, we show the redshifts, masses, and mass uncertainties of the used clusters.

The cluster masses and uncertainties are derived with $T_{X}-M$ or $L_{X}-M$ meaurements (Kettula et al., 2013; Hilton et al., 2012) (see details also in Z15). $R_{200}$ is derived with the $T_{X}-R_{200}$ relation from Arnaud et al. (2005).

\subsubsection{The DES Photometric Data}

We use the DESDM photometric catalog produced from DES coadded images with the SExtractor software. Detection threshold is set at $1.5 \sigma$ (DETECT_THRESH $=1.5)$ with the default SExtractor convolution filter. The minimum detection area is set at 6 pixels ${ }^{1}$ (DETECT_MINAREA =6). The runs were performed in dual mode, using the linear addition of $r, i$ and $z$ band images as the detection image.

A benchmark galaxy and stellar object sample is subsequently derived with the initial detections (see Bonnett et al., 2015, and also Rykoff et al. in prep., for more details), keeping only regions that are available in all of the $g, r, i, z$ bands. Regions with high density of outlier colors due to the impact of scattered light, satellite or airplane trails, and regions with low density of galaxies near the edge of the survey are removed. Objects near bright stars selected from The Two Micron All Sky Survey (2MASS Skrutskie et al., 2006) are also masked. The masking radius scales with stellar brightness in $J$ as $150-10 J$ (arcseconds) with a maximum of 120 arcseconds. Star masks with radius less than 30 arcseconds are discarded to avoid excessive masking. Coverage of the sample is recorded with the HEALPix ${ }^{2}$ software (Górski et al., 2005) gridded by $N=4096$. Photometry are re-calibrated and extinction-corrected using the Stellar Locus Regression technique (SLR:

\footnotetext{
${ }^{1}$ DECam pixel scale $0.263 "$

${ }^{2} \mathrm{http} / / /$ healpix.sourceforge.net
} 
MacDonald et al., 2004; Ivezić et al., 2007; High et al., 2009; Gilbank et al., 2011; Desai et al., 2012; Kelly et al., 2014).

For this work, we use the SExtractor Kron magnitude (mag_auto) measurements (Kron, 1980) for all detected objects. Since the SExtractor run was performed in dual mode, the Kron aperture and the centroid for different filters are the same, which are determined from the detection images. This work analyzes $z$-band luminosity function. We use any object $>10 \sigma$ in $z$ (which corresponds to magerr_auto $<0.218 \mathrm{mag}$ ). Because classifying stars/galaxies becomes inefficient beyond $22 \mathrm{mag}$ in $z$ - band, we do not attempt to separate stars and galaxies. We nevertheless refer to all the catalog objects as "galaxies" in this chapter.

\subsection{Red Sequence Galaxy Selection}

\subsubsection{Window Color Selection}

We select red sequence galaxies according to $g-r$ color at $z<0.5$, and then $r-i$ color at $0.5<z<1.05$ to account for the shifting of the $4000 \AA$ break.

For a cluster at redshift $z$, we first apply K-corrections (details explained below) to all objects in the cluster field that corrects $g-r$ or $r-i$ colors to $z=0.3$. We compare the corrected colors to a model color at $z=0.3$ with the following selection standard:

$$
\begin{array}{r}
\left|(g-r)_{z=0.3}-(g-r)_{\text {model }}\right|<\sqrt{\delta_{g-r}^{2}+\Delta_{g-r}^{2}}, \text { for } z \leq 0.5, \\
\left|(r-i)_{z=0.3}-(r-i)_{\text {model }}\right|<\sqrt{\delta_{r-i}^{2}+\Delta_{r-i}^{2}}, \text { for } z>0.5 .
\end{array}
$$

In this equation, $(g-r)_{\text {model }}$ and $(r-i)_{\text {model }}$ are the model colors (details explained below) that serve as the mid-points of the selection windows. $\delta_{g-r}$ and $\delta_{r-i}$ are the color measurement uncertainties. $\Delta_{g-r}$ and $\Delta_{r-i}$ are the width of the selection windows.

We set $\Delta_{g-r}$ to be $0.2 \mathrm{mag}$. This clipping width is chosen to encompass the intrinsic scatter and the slope of red sequence color-magnitude relations, while avoiding selecting a significant amount of blue galaxies. When selecting galaxies with $r-i$ colors at $z>0.5$, the selected populations should be consistent with those $z<0.5$. To achieve this, we optimize $\Delta_{r-i}$, the clipping width in $r-i$, so that the number counts of selected cluster galaxies with the $r-i$ standard and the $g-r$ standard are equal: $N_{\Delta r-i}=N_{\Delta_{g-r}}$.

We first perform selections with the $g-r$ standard $\left(\Delta_{g-r}\right.$ set at $\left.0.2 \mathrm{mag}\right)$, and record the number count of the selected galaxies as $N_{\Delta_{g-r}=0.2 \mathrm{mag}}$ (field contribution eliminated, 

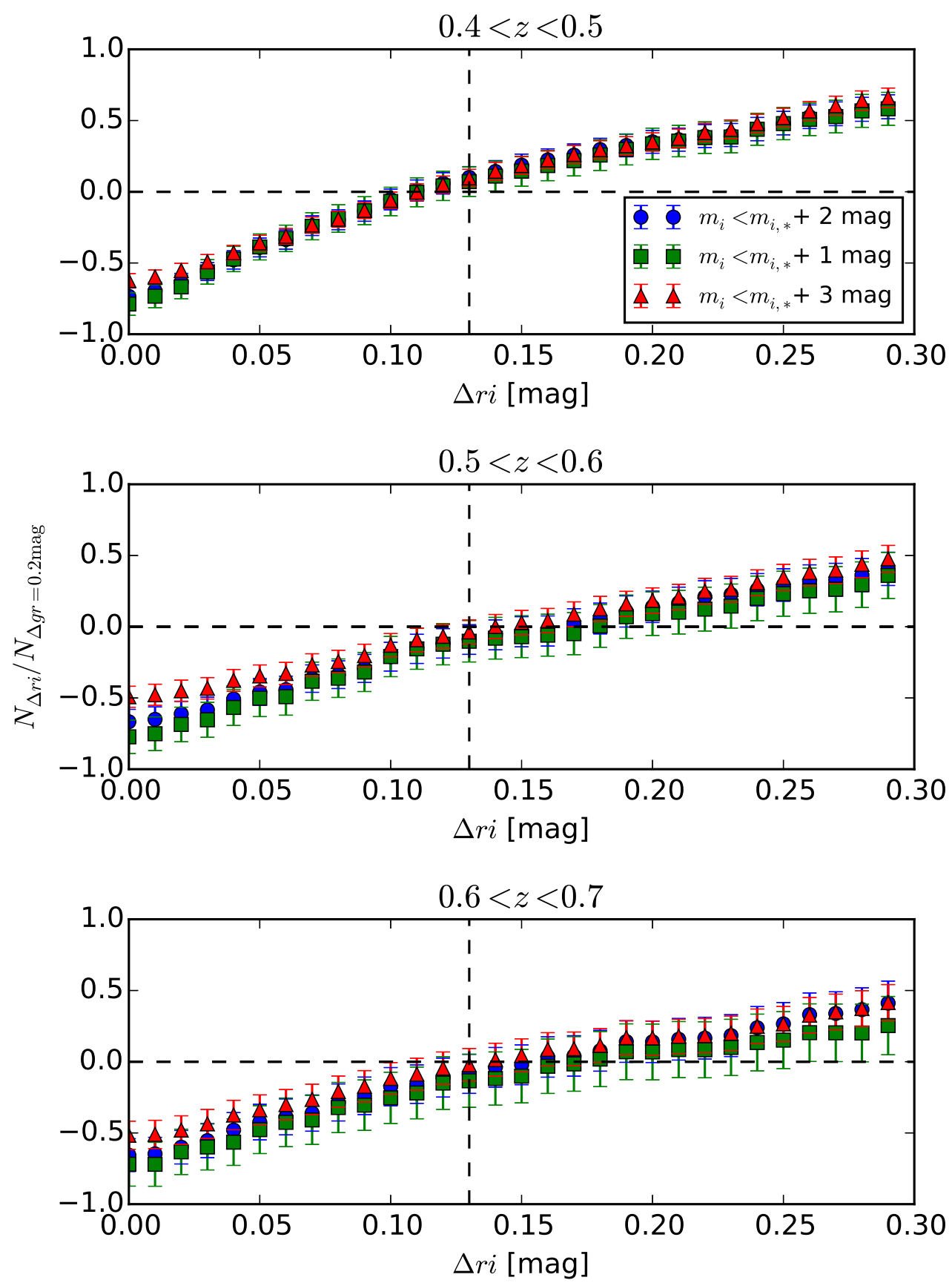

Figure 4.2: We select red sequence galaxies with $g-r$ color at $z<0.5$, and $r-i$ color at $z>0.5$. Having chosen the clipping width in $g-r$ to be 0.2 mag, we adjust the clipping width in $r-i$ to match the number of selected cluster galaxies. This figure shows how the clipping width in $r-i$ changes cluster galaxy counts in comparison to selecting with $g-r$. See Section 4.3 for details. Different symbols (circle, square and triangle) indicates cluster galaxy counts to different magnitude limits. We choose the clipping width in $r-i$ to be 0.13 (vertical dashed lines) according to this test. 
see explanations on field and cluster galaxies in Section 4.5). Then for the same clusters, we perform the selections with the $r-i$ standard while adjusting the value of $\Delta_{r-i}$, and compute the number count of the selected cluster galaxies, $N_{\Delta r-i}$ (Figure 4.2). We find

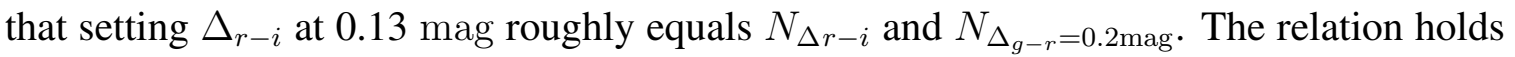
for almost all the clusters below redshift 0.9 (showing the redshift range of 0.4 to 0.7 in Figure 4.2) and for counting cluster galaxies to differnt magnitude limits.

The K-correction terms and the model colors are computed with a simple stellar population template from Bruzual \& Charlot (2003), assuming a single star burst of metallicity $Z=0.008$ at $z=3.0$, utilizing the EZGal package ${ }^{3}$ (Mancone \& Gonzalez, 2012).

\subsubsection{RedMaPPer Member Selection}

In this chapter, we also select red sequence galaxies with the RedMaPPer (Rykoff et al., 2014, and also Rykoff et al. in prep. for adaption to DES data) algorithm. This algorithm uses clusters with spectroscopic redshifts to calibrate the values and the intrinsic scatters of the red sequence colors. It then assigns a red sequence probability to each galaxy in the cluster field. Objects with red sequence probability below $1 \%$ are pruned from the final catalog. The computation of the probability considers the galaxy density profile, galaxy luminosity function and galaxy colors. Note that although a galaxy luminosity function ( $\alpha=-1$, see Rykoff et al. in prep. for details) is assumed when computing the red sequence probability, the probability is more sensitive to the colors because of the narrow intrinsic color scatter (roughly $0.025 \mathrm{mag}$ for $g-r$ and $0.015 \mathrm{mag}$ for $r-i$ ).

\subsection{Completeness Function}

\subsubsection{The Completeness Function Model}

The completeness function models the detection probability of objects in terms of their apparent magnitude. In this chapter, the completeness function is defined to answer the following question: given a real object of truth magnitude $m$, what is the probability of observing one object with observed magnitude $m$ ?

We model the completeness probability with a complementary error function of three parameters:

$$
p(m)=\lambda \frac{1}{2} \operatorname{erfc}\left(\frac{m-m_{50}}{\sqrt{2 w}}\right) .
$$

\footnotetext{
${ }^{3}$ http://www.baryons.org/ezgal/
} 


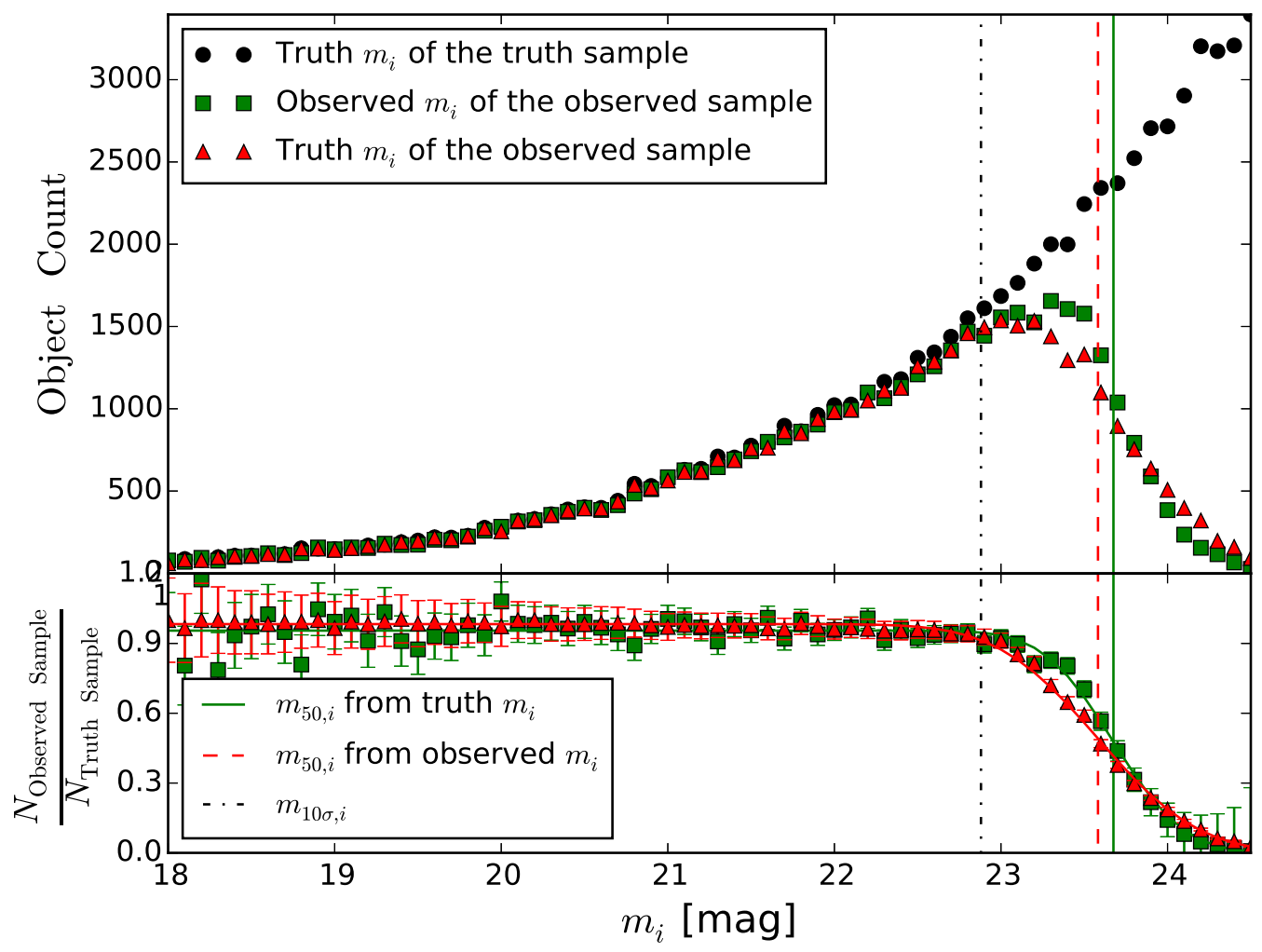

Figure 4.3: This figure demonstrates our procedure on evaluating the completeness function with the UFIGsimulation. We use a completeness function to model the difference between the observed magnitude distribution (green squares in top panel) of observed objects and the truth magnitude distribution of all truth objects (black circles in top panel). Given a truth object of truth magnitude $m$, we model the probability of observing an object with observed magnitude $m$ (green squares in lower panel), which is well described by a complementary error function. For comparison, we also show the truth magnitude distribution of the observed objects (red triangles). 

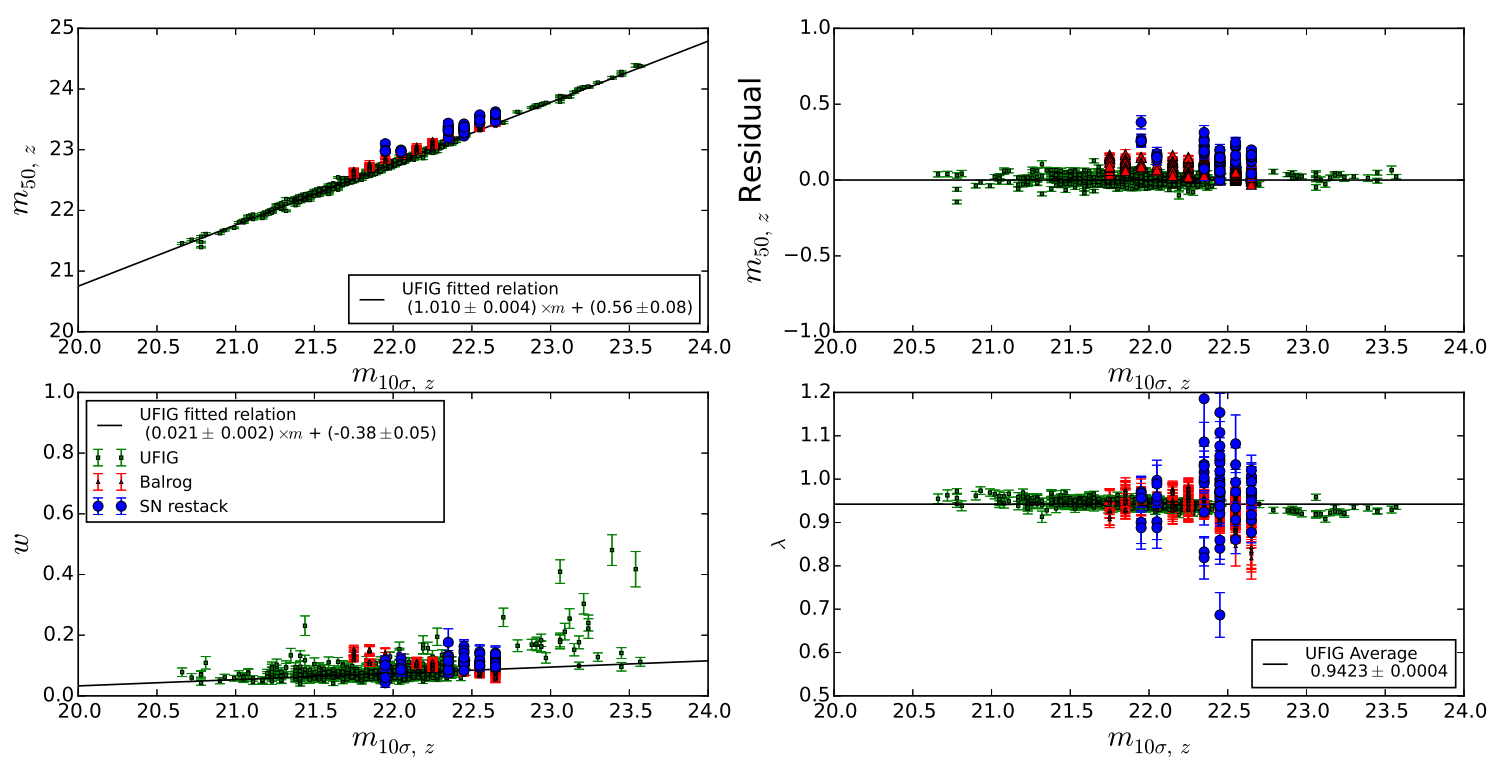

Figure 4.4: This figures shows the relations between completeness function parameters and image depth, characterized by $10 \sigma$ limiting magnitude. The relations derived from the UFIG simulation generally agree with those from the BALROG simulation. The $m_{50}$ values evaluated from re-stacking deep supernovae data appear to be 0.1-0.2 mag deeper than the relations derived from sky simulations, but the differences can be explained by Kron magnitude bias shown in Z15. Therefore, we use the relations from the UFIG simulation to model completeness function in this chapter.

In the above equation, $m_{50}$ is the $50 \%$ completeness magnitude, $w$ controls the steepness of the the detection drop-out rate and $\lambda$ is the overall amplitude of the completeness function. We further assume linear dependence of $m_{50}$ and $w$ on the depth of the image, which is characterized by the $10 \sigma$ limiting magnitude (where magerr_auto $=0.25 \times \ln 10$, or magerr_auto $=0.218$ mag assuming flux measurement, at a significance level of $10 \sigma$ ).

\subsubsection{Relations between Model parameters and Image Depth}

The $m_{50}-m_{10 \sigma}$ and $w-m_{10 \sigma}$ relations are evaluated with simulated DES images and real data. We have utilized two simulations in this procedure. The relations listed in Figure 4.4 are derived from the UFIG simulation (Bergé et al., 2013; Chang et al., 2015), which is a sky simulations that is further based on an N-body dark matter simulation populated with galaxies from the Adding Density Determined GAlaxies to Lightcone Simulations (ADDGALS) algorithm (see Lin et al., 2010; Busha et al., 2013; Dietrich et al., 2014, for an overview, Wechsler et al. in prep., Busha et al. in prep.). We use the UFIG product that matches the footprint of the benchmark sample in Section 4.2.3. The simulation is divided into fields of $0.53 \mathrm{deg}^{2}$, with the characteristic quantities like depth and seeing matching 
those of the corresponding DES-SV patches. SExtractor is applied to the simulated images with identical DES-SV setting. We select objects with magerr_auto $<0.218$ mag in $z$, derive their observed magnitude distribution, and compare it to the truth magnitude distribution of all input truth objects. The ratio between the two is well described by Equation 4.2. The derived $m_{50}$ and $w$ are tightly related to the depth of the image as shown in Figure 4.4.We also perform the analysis on the BALROG simulation (Suchyta et al., 2015), which inserts simulated objects into real DES-SV images. The results are similar.

To further verify the derived relations, we utilize restacks of high quality images from

the DES Supernovae survey with total exposure time of $\sim 1000 s$ to mimic main survey depth. The $z$-band depth of the restacks ranges from 21.5 mag to 22.5 mag, comparing to $>24$ mag coadding all eligible exposures. We compare the object counts in this set of coadds and the coaddition of all available exposures to evaluate $m_{50}$ and $w$ (also shown in Figure 4.4). The $m_{50}$ appears to be 0.1 - 0.4 mag deeper than the simulation relations, but this effect is consistent with mag_auto biases shown in Z15. In this test, we use the observed Kron magnitudes rather than the "truth" magnitudes (which is not known) from the deeper stack. Z15 shows that the observed Kron magnitudes can be biased fainter by 0.1 to 0.4 mag comparing to the "truth" mag at $<24$ mag, consistent with this test result.

Figure 4.4 indicates that the normalization of the complementary error function is lower than 1 in UFIG and BALROG. Object detection appears to be incomplete even at the bright end. This effect is caused by the same photometry measurement bias discussed above as objects are measured fainter.

We notice hints that the completeness function in galaxy clusters are different from that of the fields, possibly because of blending and larger sizes of cluster galaxies. We test this effect with simulated objects (galaxies and unresolved point sources) inserted into galaxy clusters identified with the redMaPPer algorithm $(\sim 250$ clusters in the redshift range of $[0.3,0.5])$, Figure 4.5$)$. We see evidence that $m_{50}$ inside galaxy clusters shift by $\sim 0.1 \mathrm{mag}$ comparing to fields of equivalent depth. Because the simulated galaxy sample is small, we are unable to characterize the distribution of the shifts. Therefore, we do not attempt to correct $m_{50}$ in this work.

\subsubsection{Magnitude Limits of the LF Analyses}

We determine the magnitude limits of the LF analyses according to the completeness functions. We perform the analyses only with galaxies brighter than the following magnitude limit: $m_{\lim }=m_{50}-2 w$. This cut ensures detection probability above $99.8 \% \times \lambda$ for the selected galaxies. 


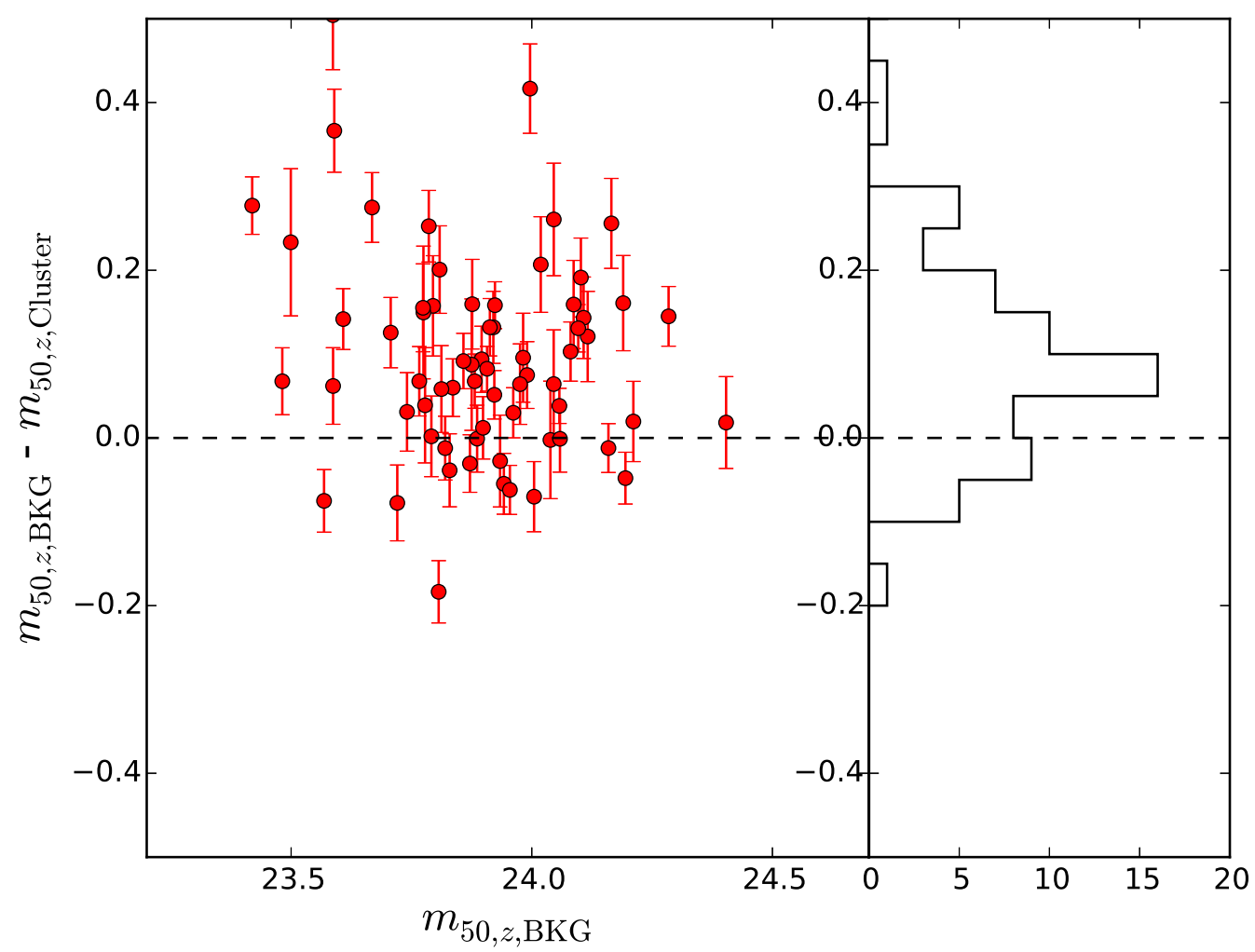

Figure 4.5: We evaluate the $m_{50}$ parameter for cluster and field regions of the same depth with the BALROG simulation. There appears to be a trend that the $m_{50}$ of the cluster regions are shallower by $\sim 0.1 \mathrm{mag}$, although the result is not well constrained due to small sample size. 


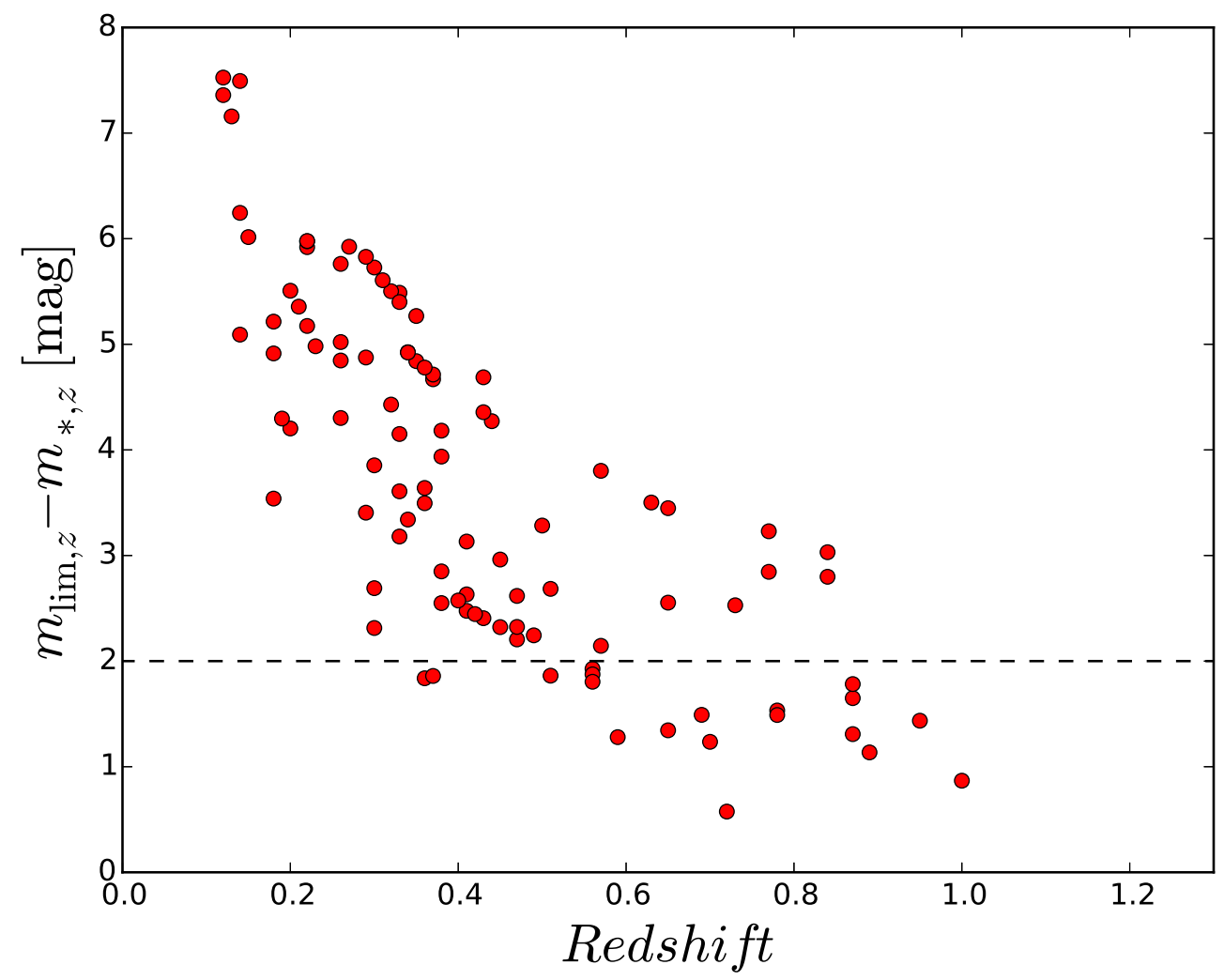

Figure 4.6: For each cluster, we derive a completeness magnitude limit, $m_{\text {lim }}$ from the completeness function. Most of the $z<0.4$ clusters are complete to $m_{z}^{*}+2$ mag and beyond. This is also true for more than $2 / 3$ of the clusters at $z>0.4$. 
In addition, if the completeness function parameters around clusters follow different relations as discussed in the above paragraph, the detection probability of the sample is still high (lower limit of $99 \% \times \lambda$ instead of $99.8 \% \times \lambda$ ).

For most of the $z<0.4$ clusters, $m_{\lim }$ is more than 2 mag fainter than the characteristic magnitude measured in Hansen et al. (2009). This is also true for more than $2 / 3$ of the clusters at $z>0.4$. We do not consider detection completeness probability in the rest of the chapter as the selected galaxy samples are highly complete.

\subsection{Methods}

In this work, we constrain the luminosity function for clusters of different masses and redshifts. We model the luminosity distribution with a single Schechter function (Schechter, 1976) up to the magnitude limit of $m_{*}+3 \mathrm{mag}$. The luminosity function are constrained with four different methods. One is based on computing histograms. Two others are developed under the Bayesian framework. The fourth one is applied to redMaPPer selected red sequence galaxies. Details of these methods are elaborated in the following sub-sections.

Generally, the input of our methods include the observed magnitudes, $\left\{m_{i}\right\}$, of objects inside galaxy clusters or in a "field" region. We define the cluster region as enclosed within $0.6 R_{200}$ of the cluster centers (BCG centers). We choose the field region to be annular, centered on the cluster, with the inner and outer radius being $3 R_{200}$ and $8 R_{200}$ respectively. The central galaxies selected in Z15 are eliminated from the analysis as they are known to be outliers to a Schechter function distribution.

The area of these region are traced with random points that cover the benchmark sample footprint. For each cluster, we generate $\sim 1.5$ million random points within $10 R_{200}$ of the cluster center. The number is high enough that the resulting uncertainty is negligible $(\sim 1 \%)$. We therefore ignore the uncertainties from using random points.

\subsubsection{The histogram method}

We first utilize a histogram approach that counts object numbers in magnitude bins. We use 150 bins from 15 mag to 30 mag spaced by 0.1 mag. We do not see significant change of the results when adjusting the bin size in the range of $0.2 \mathrm{mag}$ to $0.05 \mathrm{mag}$.

The histogram counting is performed for the cluster region, $N(m)$, and the field region, $N(m)_{\text {background }}$. To estimate the contribution of field galaxies to the cluster histogram, we 

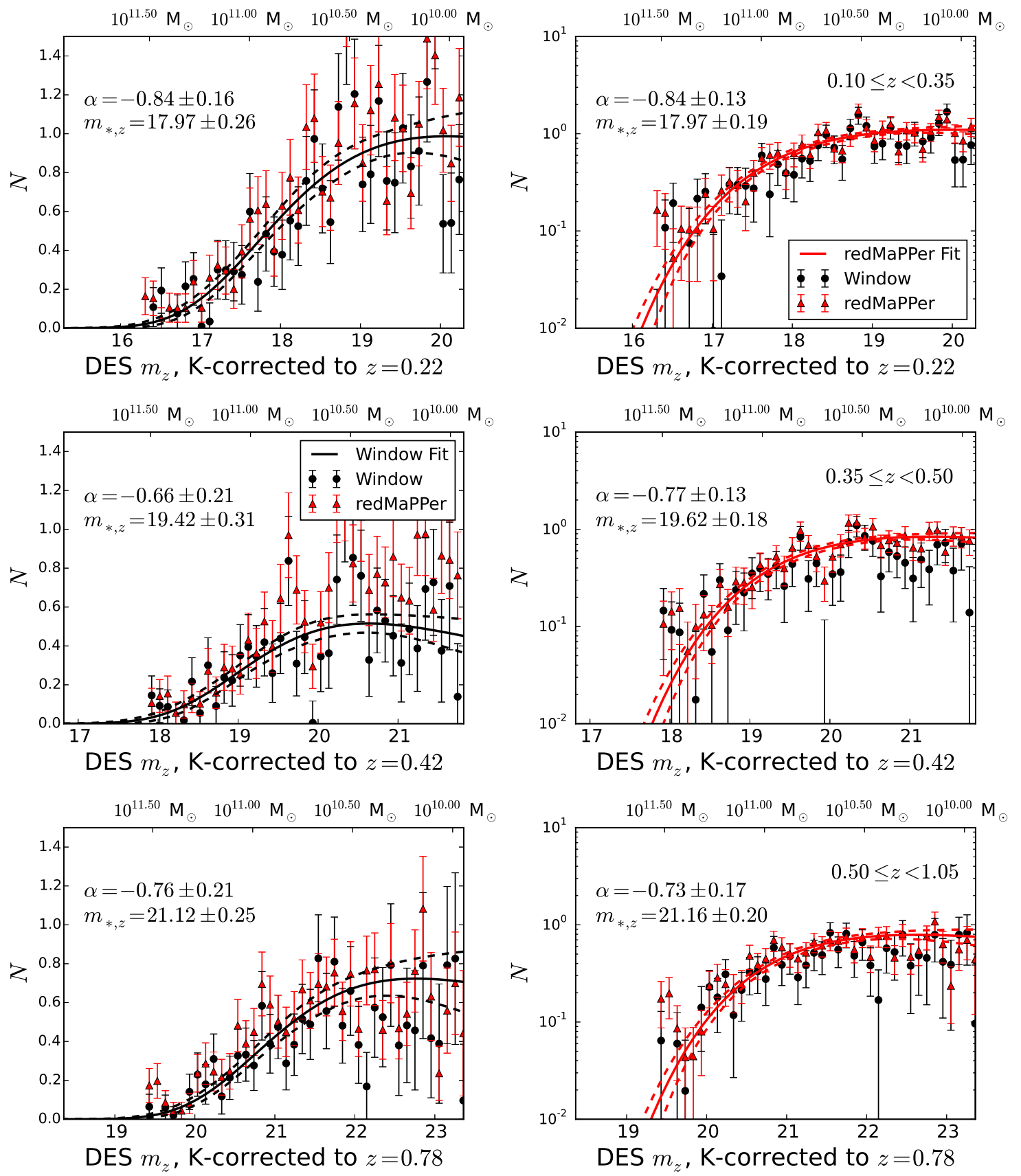

Figure 4.7: Cluster red sequence lumnosity function derived in redshift bins for the window color selection (black filled circles) and redMaPPer member selection (red squares). The left column shows cluster galaxy magnitude distribution at linear scale. The right column shows the same data but at log scale. We also plot the Schechter function fitting results for the window color selected galaxies (black solid and dashed lines in the left column) and for the redMapper selected members (red solid and dashed lines in the right column). 

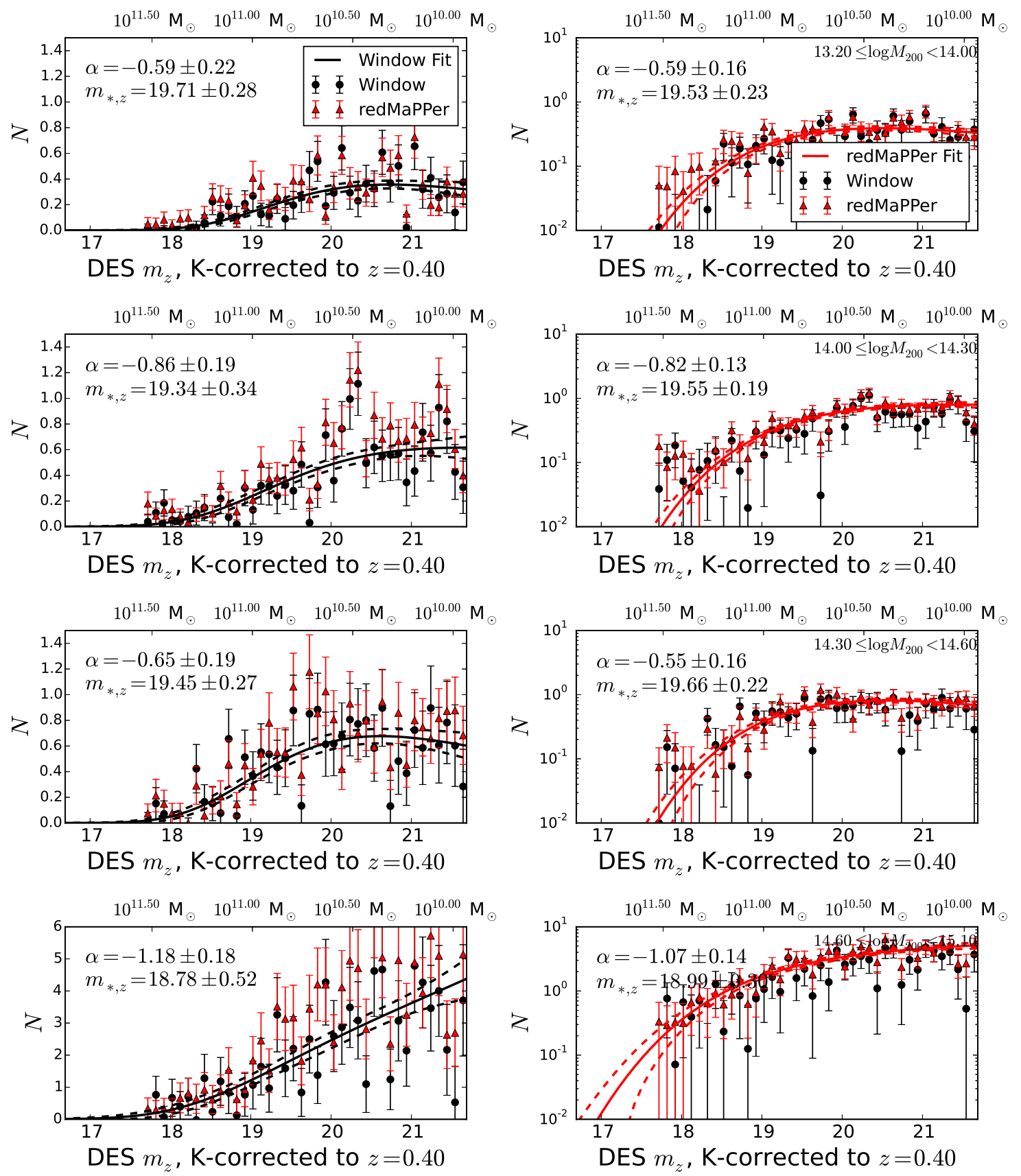

Figure 4.8: Cluster red sequence lumnosity function derived in $M_{200}$ bins for the window color selection (black filled circles) and redMaPPer member selection (red squares). The left column shows cluster galaxy magnitude distribution at linear scale. The right column shows the same data but at log scale. We also plot the Schechter function fitting results for the window color selected galaxies (black solid and dashed lines in the left column) and for the redMapper selected members (red solid and dashed lines in the right column). 
weight the number count of objects in the field region, with the random number ratio:

$$
N_{\text {bg }}(m)=N(m)_{\text {background }} \times \frac{N_{\text {random, cluster }}}{N_{\text {random, background }}} .
$$

We add up the histograms of clusters binned by redshifts (Figure 4.7) or cluster masses (Figure 4.8), and also record the number count of clusters in each magnitude bin, $C(\mathrm{~m})$. The histograms are then averaged for both the cluster region and the field region to obtain $\bar{N}(m)$ and $\bar{N}_{\text {bg }}(m)$.

We assume a Schechter function distribution for cluster galaxies

$$
S(m)=\phi(0.4 \ln 10) 10^{0.4\left(m^{*}-m\right)(\alpha+1)} \exp \left(-10^{0.4\left(m^{*}-m\right)}\right)
$$

therefore the expected number of galaxies in each magntiude bin in the cluster region is

$$
E(m)=S(m)+N_{\mathrm{bg}}(m)
$$

Assuming Poisson distribution for the total number of galaxies in each bin, we sample from the following likelihood (with the EMCEE package: Foreman-Mackey et al., 2013):

$$
\log \mathcal{L} \propto \sum_{m} \bar{N}(m) C(m) \log [E(m) C(m)]-E(m) C(m)
$$

\subsubsection{The Bayesian Inference methods}

Given a model with a set of parameters $\Omega$ that describe the distribution of observables, Bayesian theory provides a framework for inferring $\Omega$ with a set of observed quantities $\{x\}$. In this sub-section, we describe additional methods developed in this framework.

Denoting the probability of observing $\{x\}$ in model $\Omega$ to be $P(\{x\} \mid \Omega)$, and the prior knowledge about the model parameters to be $P(\Omega)$, after observations of $\{x\}$, the Bayesian theory updates the knowledge about model parameters, namely the posterior distribution, to be:

$$
P(\Omega \mid\{x\}) \propto P(\{x\} \mid \Omega) P(\Omega)
$$

The above equation uses a proportional sign instead of an equal sign as a probability function needs to be normalized to 1 . In practice, the normalization factor is almost always

un-interesting because the probability can be sampled with Markov chain Monte Carlo without knowing it.

In the Bayesian inference framework, constraining a model follows these steps: 
1. coming up with a model (with a few free parameters) that describe the observables, and

2. writing the probability of observing the observables in the model.

In our application, the observables include the observed magnitudes of objects in the cluster or field region. A major component of our models is the Schechter function. We develop two models that describe the cluster galaxy distribution of a cluster sample. The first model, called the binning model, resembles the histogram approach where the faint end slope and characteristic magnitude are fixed to be the same for all clusters in one redshift/mass bin. The second model, called the dependence model, assumes redshift and mass dependences for the faint end slope and characteristic magnitude, bypassing the binning step.

\subsubsection{Basic components of the models}

The Basic components that describe one galaxy cluster are the same for both of the models.

For one cluster galaxy, we assume that the probability of observing it with magnitude $m$ follows a Schechter function:

$$
f(x)=\psi_{f}(0.4 \ln 10) 10^{0.4\left(m^{*}-x\right)(\alpha+1)} \exp \left(-10^{0.4\left(m^{*}-x\right)}\right)
$$

In this equation, $\psi_{f}$ is the normalization parameter that normalizes $f(x)$ to $1 . \alpha$ and $m^{*}$ are the faint end slope and characteristic magnitude, treated as free parameters of the model.

For one object in the cluster region, it can be either a cluster galaxy or a field object. For a field object, we denote the probability of observing it with magnitude $m$ to be $g(m)$. $g(m)$ is approximated with a normalized histogram of the object magnitude distribution in the field region.

The probability of observing one object in the cluster region is the combination of observing it as a field object and observing it as a cluster galaxy. The probability writes as

$$
h(m)=\psi_{h}\left[N_{\mathrm{cl}} f(m)+N_{\mathrm{bg}} g(m)\right]
$$

In this equation, $N_{\mathrm{cl}}$ is the number of cluster galaxies in the cluster region, and $N_{\mathrm{bg}}$ is the number of field galaxies in the cluster region. Again, there exists a normalization factor $\psi_{h}$ that normalizes the probability function to 1 .

$N_{\text {bg }}$ can be easily extrapolated from the field region and the area ratio between the cluster and the field regions, with negligible uncertainty. We therefore treat $N_{\text {bg }}$ as a known

quantity. Equation 4.9 introduces only one additional free parameter, $N_{\mathrm{cl}}$, which controls 
the relative density between cluster and field galaxies in the cluster region. $N_{\mathrm{cl}}$ can be further related to the amplitude of the Schechter function, $\phi^{*}$ (in unit of total galaxy count), as the integration of the Schechter function over the interested magnitude range, writing as

$$
\begin{aligned}
N_{\mathrm{cl}} & =\int \frac{\phi^{*} f(m)}{\psi_{f}} \mathrm{~d} m \\
& =\frac{\phi^{*}}{\psi_{f}} \int f(m) \mathrm{d} m .
\end{aligned}
$$

Thus far, the free parameters in our models are $\alpha, m^{*}$ from Equation 4.8 and $\phi^{*}$.

We further constrain $\phi^{*}$ with the number count of observed objects in the cluster region $(N)$, assuming a Poisson distribution:

$$
N \sim \mathcal{P} \operatorname{oisson}\left(N_{\mathrm{cl}}+N_{\mathrm{bg}}\right)
$$

The log-likelihood explicitly writes as:

$$
\log \mathcal{P}(N) \propto N \log \left(N_{\mathrm{cl}}+N_{\mathrm{bg}}\right)-\left(N_{\mathrm{cl}}+N_{\mathrm{bg}}\right)
$$

For one cluster, we take the observables to be the observed magnitudes of cluster region objects, $\left\{m_{i}\right\}$, and the object number count $N$. The log-likelihood of observing these quantities writes as

$$
\begin{aligned}
\log \mathcal{P}( & \left.\left\{m_{i}\right\}, N \mid \alpha, m^{*}, \phi^{*}\right) \\
& \propto \log \mathcal{P}\left(N \mid \phi^{*}, \alpha, m^{*}\right)+\sum_{i} \log \mathcal{P}\left(\left\{m_{i}\right\} \mid \alpha, m^{*}, \phi^{*}\right) \\
& \propto \log \mathcal{P}(N)+\sum_{i} \log h\left(m_{i}\right) .
\end{aligned}
$$

\subsubsection{The Binning Model}

As most of the clusters in our sample have low masses, a single cluster will not yield statistically significant constraints on $\alpha, m^{*}, \phi^{*}$. We therefore bin the clusters by their redshifts or masses, and assume $\alpha, m^{*}$ to be the same for one cluster bin. $\phi^{*}$ are constrained independently for each cluster. For one cluster bin, the model parameters are $\alpha, m^{*}$, and $\left\{\phi_{j}^{*}\right\}$. The log-likelihood of observing these quantities writes as: 


$$
\begin{aligned}
\log \mathcal{L}\left(\left\{m_{i, j}\right\},\left\{N_{j}\right\} \mid \alpha, m^{*},\left\{\phi_{j}^{*}\right\}\right) \\
\propto \sum_{j}\left[\log \mathcal{P}\left(N_{j} \mid \phi_{j}^{*}, \alpha, m^{*}\right)+\sum_{i} \log \mathcal{P}\left(\left\{m_{i, j}\right\} \mid \alpha, m^{*}, \phi_{j}^{*}\right)\right] \\
\propto \sum_{j}\left[\log \mathcal{P}_{j}\left(N_{j}\right)+\sum_{i} \log h_{j}\left(m_{i}, j\right)\right] .
\end{aligned}
$$

The posterior likelihood of $\alpha, m^{*},\left\{\phi_{j}^{*}\right\}$ is the combination of their prior likelihood and $\log \mathcal{L}\left(\left\{m_{i, j}\right\},\left\{N_{j}\right\} \mid \alpha, m^{*},\left\{\phi_{j}^{*}\right\}\right)$, writing as

$$
\begin{aligned}
& \log \mathcal{L}\left(\alpha, m^{*},\left\{\phi_{j}^{*}\right\} \mid\left\{m_{i, j}\right\},\left\{N_{j}\right\}\right) \\
& \quad \propto \log \mathcal{L}\left(\alpha, m^{*},\left\{\phi_{j}^{*}\right\}\right)+\log \mathcal{L}\left(\left\{m_{i, j}\right\},\left\{N_{j}\right\} \mid \alpha, m^{*},\left\{\phi_{j}^{*}\right\}\right) .
\end{aligned}
$$

We assume a flat prior between $[-4,4]$ for $\alpha$ and $[-10,10]$ for $\left\{\phi_{j}^{*}\right\}$. We assume a Gaussian distribution for $m^{*}$, with the mean set to be the measurement from De Propris et al. (2013), and variance set to be $1 \mathrm{mag}^{2}$. This prior is only important for the highest redshift bin.

\subsubsection{The Dependence Model}

Instead of binning clusters into redshift/mass bins, it is possible to model the cluster redshift/mass dependences directly. We rewrite $\alpha$ and $m^{*}$ as

$$
\begin{aligned}
\alpha_{j} & =A_{\alpha} \log \left(1+z_{j}\right)+B_{\alpha}\left(\log M_{\text {true }, j}-14\right)+C_{\alpha} \\
m_{z=0.4, j}^{*} & =B_{m}\left(\log M_{\text {true }, j}-14\right)+C_{m} .
\end{aligned}
$$

In the above equations, $\log M_{\text {true }, j}$ represents the truth mass of the $j$ the cluster. $\log M_{\text {true }, j}$ for different clusters are treated as free parameters in the analysis, but we use observational constraints on $\log M_{200}$ from X-ray data as priors (Gaussian distributions): $\log M_{\text {true }, j} \sim$ $\mathrm{N}\left(\log M_{\mathrm{obs}, j}, \sigma_{M}^{2}\right) . \sigma_{M}$ is the measurement uncertainty of $\log M_{\mathrm{obs}, j}$ from X-ray data. The assumption about $\log M_{\text {true }, j}$ allows us to incorporate mass uncertainties into the analysis.

Again, $\phi^{*}$ for each cluster is constrained separately. We further assume a power law relation between $M_{\text {true }}$ and $\phi^{*}$ :

$$
\log \phi^{*}=B_{\phi} \times \log M_{\text {true }}+B_{\phi}
$$

We assume a Gaussian distribution of $\left\{\log \phi_{j}^{*}\right\}$ given the values predicted by the relation: $\phi_{j}^{*} \sim \mathrm{N}\left(\log \phi_{\text {relation }}, \sigma_{\log \phi}^{2}\right) . \sigma_{\log \phi}$ is the intrinsic scatter of the relation, fixed at 0.22 (close to the constraint from further testing that allows the parameter to float) to reduce the number 
of free parameters. The $\log$ likelihood of having $\phi_{j}^{*}$ given $M_{\mathrm{true}, j}$ writes as:

$$
g_{j}\left(\phi_{j}^{*}\right) \propto-\frac{\left(\phi_{j}^{*}-\phi_{\text {relation }}\right)^{2}}{2 \sigma_{\log \phi}^{2}}
$$

The free parameters of this model are $A_{\alpha}, B_{\alpha}, C_{\alpha}, B_{m}, C_{m}, B_{\phi}, C_{\phi},\left\{\phi_{j}^{*}\right\}$ and $\left\{M_{\text {true }, j}\right\}$. The observed quantities are $\left\{m_{i, j}\right\}$ and $\left\{N_{j}\right\}$ of all clusters. The log-likelihood of observing these quantities writes as:

$$
\begin{aligned}
\log \mathcal{L}( & \left.\left\{m_{i, j}\right\},\left\{N_{j}\right\} \mid A_{\alpha}, B_{\alpha}, C_{\alpha}, B_{m}, C_{m}, B_{\phi}, C_{\phi},\left\{\phi_{j}^{*}\right\},\left\{M_{\text {true }, j}\right\}\right) \\
& =\log \mathcal{L}\left(\left\{m_{i, j}\right\},\left\{N_{j}\right\} \mid \alpha_{j}, m_{j}^{*},\left\{\phi_{j}^{*}\right\}\right)+\log \mathcal{L}\left(\left\{\phi_{j}^{*}\right\} \mid\left\{M_{\text {true }, j}\right\}\right) \\
& \propto \sum_{j}\left[\log \mathcal{P}\left(N_{j} \mid \phi_{j}^{*}, \alpha_{j}, m_{j}^{*}\right)+\sum_{i} \log \mathcal{P}\left(\left\{m_{i, j}\right\} \mid \alpha_{j}, m_{j}^{*}, \phi_{j}^{*}\right)\right] \\
& +\sum_{j} \log \mathcal{L}\left(\phi_{j}^{*} \mid M_{\text {true }, j}\right) \\
& \propto \sum_{j}\left[\log \mathcal{P}_{j}\left(N_{j}\right)+\sum_{i} \log h_{j}\left(m_{i}, j\right)+g_{j}\left(\phi_{j}^{*}\right)\right] .
\end{aligned}
$$

Again, we assume flat priors of $[-10,10]$ for $\left\{\phi_{j}^{*}\right\}$, then $[-2,2]$ for $A_{\alpha}, B_{\alpha}$ and $C_{\alpha}$, $[-10,10]$ for $B_{m}$. For $C_{m}$, we assume a Gaussian distribution for $m^{*}$, with the mean set to be the measurement from De Propris et al. (2013), and variance set to be $1 \mathrm{mag}^{2}$.

\subsection{3 redMaPPer Selected objects}

For the red sequence members selected from the redMaPPer algorithm, we use a Bayesian approach to constrain the luminosity function of clusters binned by redshifts (Figure 4.7) or masses (Figure 4.8). The procedure is similar the one described in Sectiton 4.5.2.2, despite the different methods in assessing the field contribution.

For each redMaPPer member with magnitude $m_{i}$, the posterior probability of observing it is described by the Schechter function:

$$
S\left(m_{i}\right) \propto \psi_{s}\left(\alpha, m^{*}\right) 10^{0.4\left(m^{*}-m_{i}\right)(\alpha+1)} \exp \left(-10^{0.4\left(m^{*}-m_{i}\right)}\right)
$$

where $\psi_{s}$ normalizes the integration over $m_{i}$ to 1 . The redMaPPer algorithm assigns a member probability of $p_{i}$ to each galaxy. It is necessary to weight $S\left(m_{i}\right)$ when calculating 

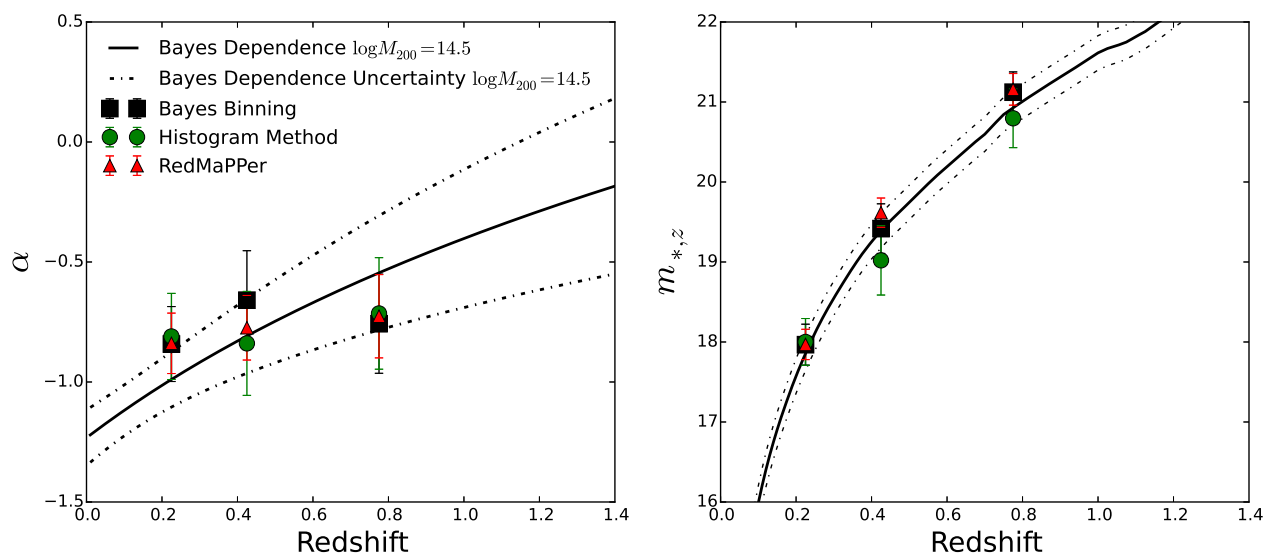

Figure 4.9: Redshift evolution of the faint end slope, $\alpha$, and the characteristic magnitude, $m^{*}$, derived with four different methods.

the posterior probability of observing $\left\{m_{i}, p_{i}\right\}$ :

$$
\log \mathcal{L}\left(\left\{m_{i}, p_{i}\right\} \mid \alpha, m^{*}\right) \propto \sum_{i} p_{i} \log S\left(m_{i}\right)
$$

The amplitude of the Schechter function, $\phi^{*}$, constrains the total number of cluster galaxies in a magnitude range:

$$
\begin{aligned}
N_{\mathrm{cl}} & =\int \frac{\phi^{*} s(m)}{\psi_{s}\left(\alpha, m^{*}\right)} \mathrm{d} m \\
& =\frac{\phi^{*}}{\psi_{s}\left(\alpha, m^{*}\right)} \int s(m) \mathrm{d} m .
\end{aligned}
$$

We take the the observed number of cluster galaxies to be the weighted total number of redMaPPer members: $N=\sum_{i} p_{i}$, and assume a Poisson distribution for $N$ with an expected value of $N_{\mathrm{cl}}$.

The final posterior likelihood for constraining $\alpha, m^{*}$ and $\phi^{*}$ becomes:

$$
\begin{aligned}
\log \mathcal{L}\left(\left\{m_{i}, p_{i}\right\}, N \mid \alpha, m^{*}\right) \\
\propto \log \mathcal{P}\left(N \mid \phi^{*}, \alpha, m^{*}\right)+\sum_{i} p_{i} \log S\left(m_{i}\right)
\end{aligned}
$$



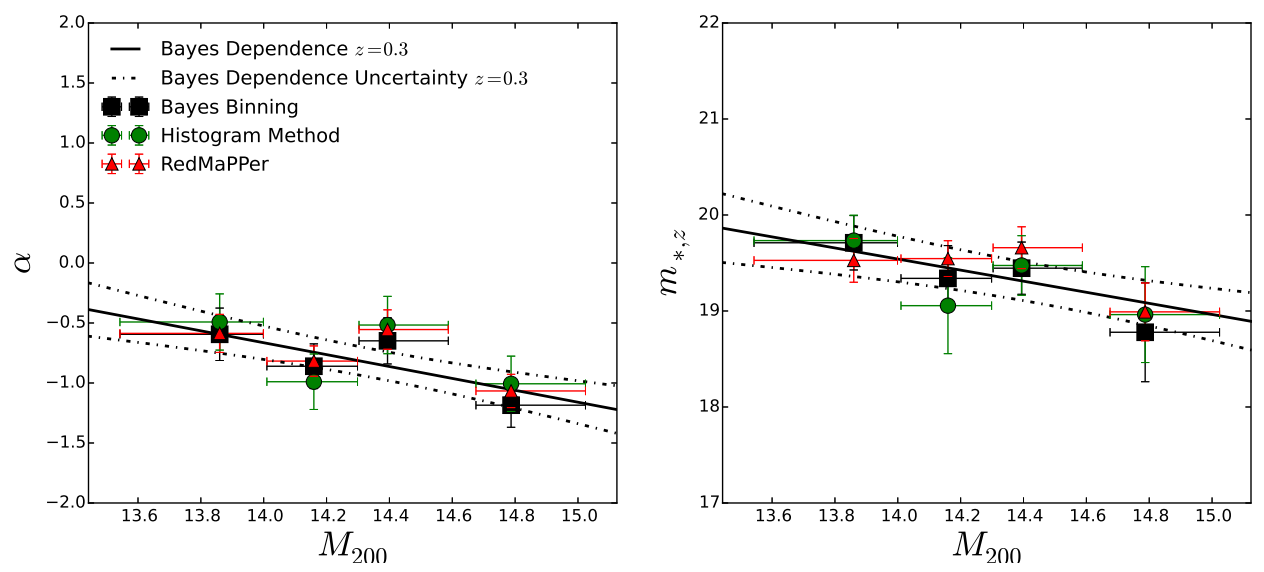

Figure 4.10: Mass dependence of the faint end slope, $\alpha$, and the characteristic magnitude, $m^{*}$, derived with four different methods.

\subsection{Results}

\subsubsection{Results in Redshift/Mass Bins}

To constrain the redshift evolution of RSLF, we select clusters with masses between $10^{14.0} M_{\odot}$ and $10^{15.0} M_{\odot}$ and bin them into three redshift bins: $0.1 \leq z<0.35,0.35 \leq$ $z<0.5$ and $0.5 \leq z$. The purpose of fixing the mass range is to reduce possible impact from RSLF mass dependence. The mass range selection is indicated in Figure 4.1. We have tested changing the mass selection range to $10^{13.8} M_{\odot}$ and $10^{14.8} M_{\odot}$, but the results are similar.

To constrain the mass dependence of RSLF, we select clusters below redshift 0.5 (the selection is also indicated in Figure 4.1) and bin them into four mass bins: $13.2 \leq \log M_{200}<$ 14.0, $14.0 \leq \log M_{200}<14.3,14.3 \leq \log M_{200}<14.4$ and $14.4 \leq \log M_{200}<15.1$. The purpose of fixing the redshift range is to reduce possible impact from RSLF redshift evolution. To reduce uncertainties from band shifting, we K-correct all galaxies to $z=0.4$ and constrain RSLF parameters at this redshift.

We then apply the Histogram method (Section 4.5.1), the Bayes binning method (Section 4.5.2.2), and the redMaPPer method (Section 4.5.3) to clusters in redshift or mass bins. For each cluster, we use galaxies in the $\left[m^{*}-2, m^{*}+2\right]$ magnitude range, estimated with the characteristic magnitude measurement from Hansen et al. (2009). If the completeness magnitude limit of the cluster, $m_{\text {lim }}$, is below $m^{*}+2$, we apply the methods only to the $\left[m^{*}-2\right.$, $\left.m_{\text {lim }}\right]$ magnitude range.

The results from the Histogram method, the Bayes binning method, and the redMaPPer method are shown in Figures 4.9 and 4.10. Overall, the three methods agree remarkably 
well.

We examine the redshift evolution of $\alpha$ through fitting a linear relation between $\alpha$ and $\log (1+z): \alpha=a \times \log (1+z)+b$. We take $z$ to be the middle values of the redshift bins. Deviation of $a$ from 0 indicates redshift evolution of $\alpha$. We derive $a$ to be $0.58 \pm 0.50$ with the results from the Histogram method, $0.57 \pm 0.99$ from the Bayes binning method, and $0.71 \pm 0.15$ from the redMaPPer method. These results suggest that it is not reliable to constrain redshift evolution with just three bins. We have attempted to implement four redshift bins, but the results are similar. On the other hand, although we have narrowed the cluster mass range in this exercise, it is possible that our results are still impacted by mass selection effect. This is evidenced by the different amplitude of the RSLF in different redshift bins (Figure 4.7).

We also examine the mass dependence of $\alpha$ through fitting a linear relation between $\alpha$ and $\log M_{200}: \alpha=a \times\left(\log M_{200}-14.0\right)+b$. Again, deviation of $a$ from 0 indicates mass dependence of $\alpha$. We take $\log M_{200}$ to be the middle values of the mass bins. We derive $a$ to be $-0.09 \pm 0.93$ with the results from the Histogram method, $-0.68 \pm 0.36$ from Bayes binning method, and $-0.22 \pm 0.14$ from the redMaPPer method. Results from the Bayes binning method hints mass dependence of $\alpha$. Similarly, we constrain the mass dependence for $m_{z, *}$ with $a$ being $-0.48 \pm 0.67,-1.0 \pm 0.78$ and $-0.15 \pm 0.06$ for the Histogram, Bayes binning and the redMaPPer methods respectively.

\subsubsection{Results from the Bayes Dependence Model}

We use the Bayes Dependence model (Section4.5.2.3) to simultaneously constrain the redshift evolution and mass dependence of $\alpha$ and mass dependence of $m_{*}$ :

$$
\begin{aligned}
\alpha & =A_{\alpha} \log (1+z)+B_{\alpha}\left(\log M_{200}-14\right)+C_{\alpha} \\
m_{z=0.4}^{*} & =B_{m}\left(\log M_{200}-14\right)+C_{m} .
\end{aligned}
$$

For each cluster, we perform the fitting only with galaxies in the $\left[m^{*}-2, m^{*}+2\right]$ magnitude range. If the completeness magnitude limit of the cluster, $m_{\text {lim }}$, is below $m^{*}+2$, we eliminate this cluster from the fitting procedure. We use a total of 77 clusters in this method. Including these clusters with galaxy selections in the $\left[m^{*}-2, m_{\text {lim }}\right]$ magnitude range do not appear to change our results.

The constraints on the $\alpha$ and $m *_{z=0.4}$ relations are listed in Table 4.1. The constraints show hints of both redshift evolution and mass dependence of $\alpha$ : the $A_{\alpha}$ parameter that controls redshift evolution deviates from 0 at a significance level of $2.6 \sigma$, while the $B_{\alpha}$ parameter that controls mass dependence deviates from 0 at a significance level of $2.4 \sigma$. 
Table 4.1: Constraints on the $\alpha$ and $m *_{z=0.4}$ relations

$\begin{array}{ccc} & \text { Prior } & \text { Posterior } \\ A_{\alpha} & {[-5,10]} & 2.3 \pm 0.9 \\ B_{\alpha} & {[-4,4]} & -0.50 \pm 0.21 \\ C_{\alpha} & {[-2,2]} & -0.93 \pm 0.13 \\ B_{m} & {[-10,10]} & -0.58 \pm 0.31 \\ C_{m} & \mathcal{N}\left(-22.15,1 \mathrm{mag}^{2}\right) & -22.28 \pm 0.24 \\ & \mathcal{N}\left(19.69,1 \mathrm{mag}^{2}\right) \text { at } z=0.4 & 19.54 \pm 0.24 \text { at } z=0.4 \\ B_{\phi} & {[-5,5]} & 0.41 \pm 0.14 \\ C_{\phi} & {[-10,10]} & 0.94 \pm 0.06\end{array}$

For clusters of $\log M_{200}=14.2$ (the median mass of our sample), we constrain $\alpha$ to be $-0.84 \pm 0.07$ at $z=0.2$, rising to $-0.52 \pm 0.12$ at $z=0.6$. For $\log M_{200}=15.0, \alpha$ is constrained to be $z=0.2$.

The dependence model assumes a passive evolution model for the RSLF characteristic magnitude in $z$-band. We do not notice evidence of $m^{*}$ deviating from the passive evolution model in this work (the results on $m^{*}$ in redshift bins agree with the interpolations from the model). However, we do notice tantalizing hint of $m^{*}$ being brighter for more massive clusters at a significance level of $1.9 \sigma$ (see constraint on $B_{m}$ in Table 4.1).

In Figures 4.9 and 4.10, we plot the $\alpha$ and $m *_{z=0.4}$ relations as well as their uncertainties. We show the redshift evolution of $\alpha$ with $\log M_{200}$ fixed at 14.5 to match the binning results, and show the mass dependence of $\alpha$ with $z$ fixed at 0.3 . The constraints from the binning approaches in Section 4.6.1 match well with the dependence model, testifying the accuracy of the dependence model results.

The Bayes dependence model also constrain the RSLF amplitudes, $\phi^{*}$, as well as the relations between $\phi^{*}$ and $\log M_{200} . \phi^{*}$ is the determinant parameter in modeling the total number of cluster galaxies. Our result shows strong correlation between $\phi^{*}$ and cluster masses (Figure 4.11).

The correlation between $\phi^{*}$ and $\log M_{200}$ may also help with constraining $\log M_{200}$. The dependence model constrain the $M_{\text {true }}$ parameters usring priors from X-ray measurements. We compare the constraints on $M_{\text {true }}$ to the X-ray measured $M_{\text {obs }}$ (Figure 4.12). For most of the clusters, $M_{\text {true }}$ agree remarkably well with $M_{\text {obs }}$. The constraints on cluster masses improves only when the uncertainties of $M_{\text {obs }}$ is larger than 0.3 dex. The result demonstrates the power of X-ray data on measuring cluster masses. 


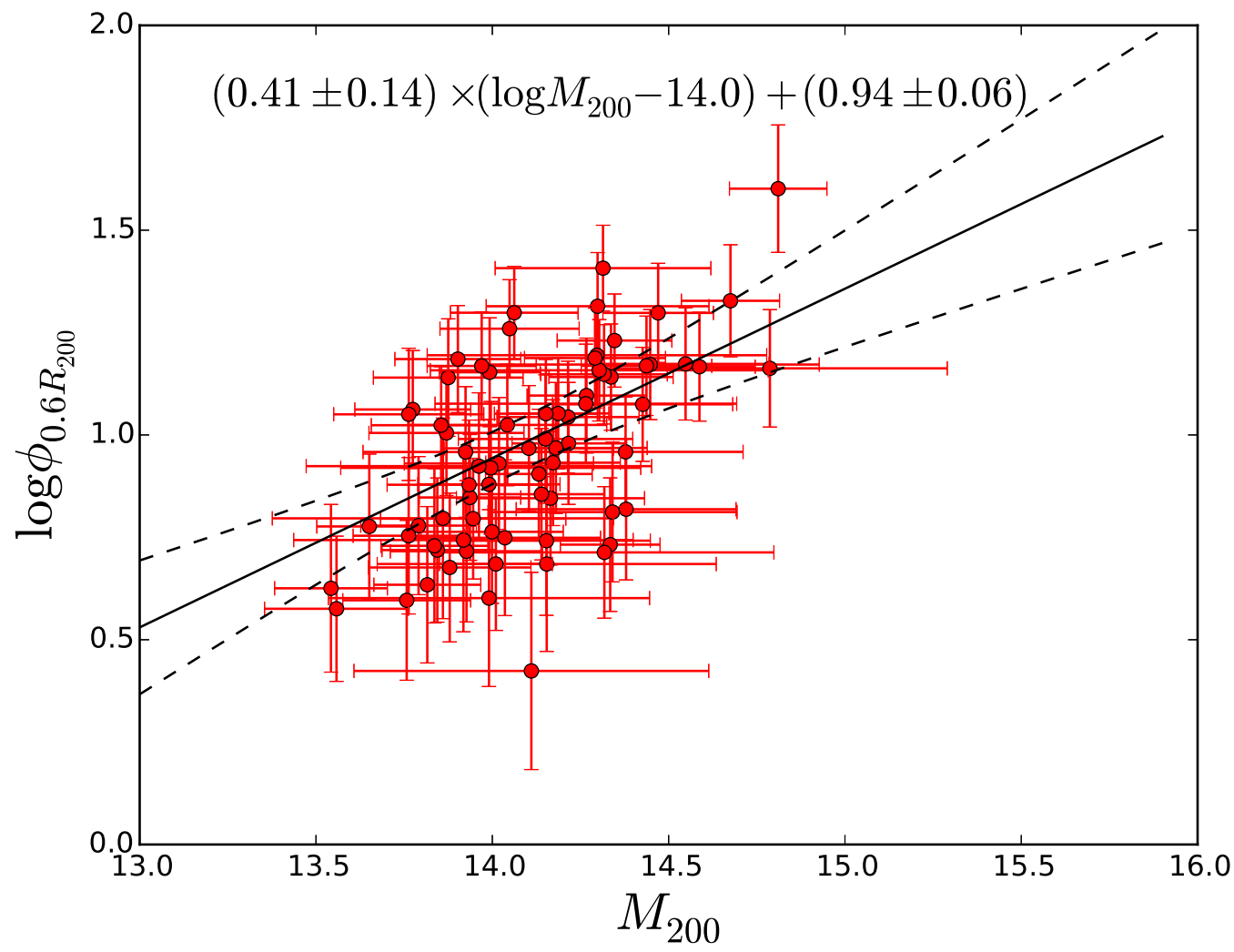

Figure 4.11: Constraints on the amplitude of the luminosity function for individual clusters. We do not observe significant redshift evolution effect of the $\phi^{*}-M_{200}$ relations, therefore we only model it as mass dependent in this work. The linear relation between $\phi^{*}$ and $M_{200}$ is determined at the same time in the Bayes dependence method (Section4.5.2.3). 

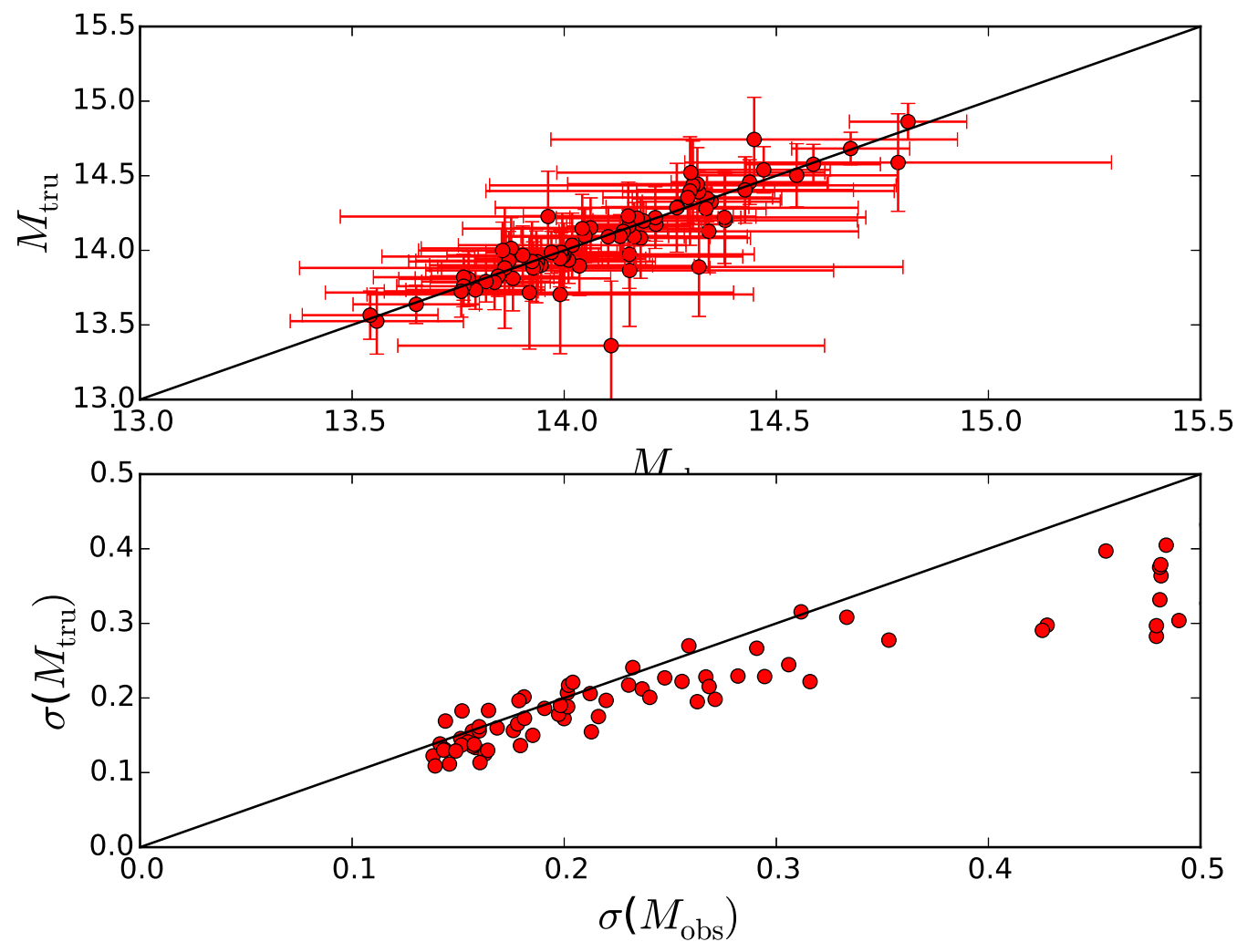

Figure 4.12: In the Bayesian dependence model, we probe cluster mass using priors from $\mathrm{X}$-ray measurements. The constrained cluster masses generally agree with X-ray measurements. The mass uncertainty improves when the X-ray measurement uncertainty, $M_{\mathrm{obs}}$, is larger than 0.3 dex.
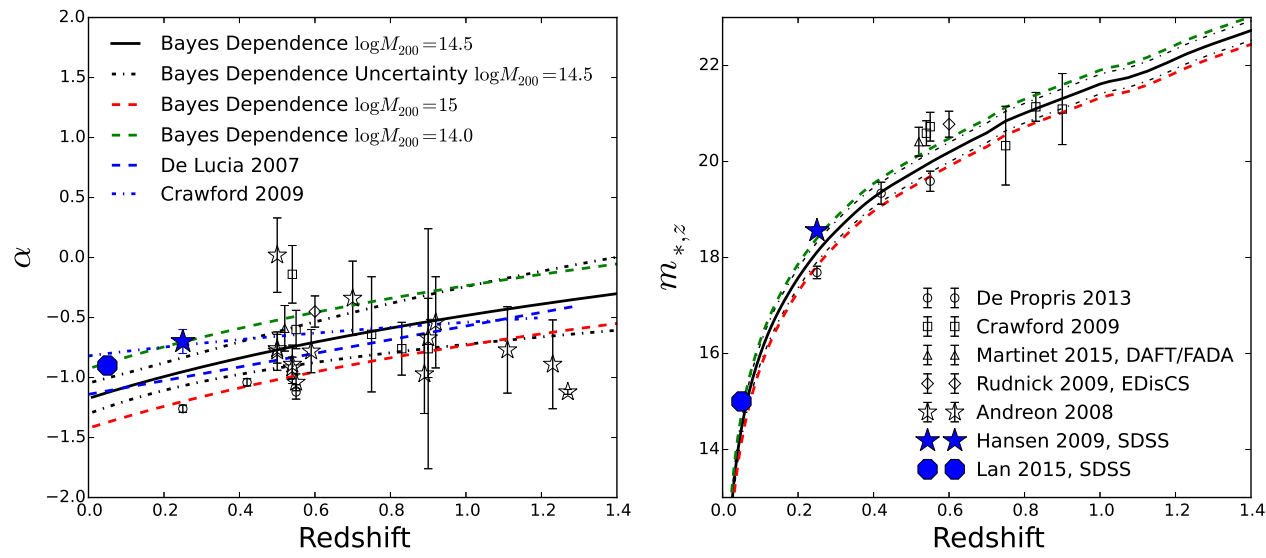

Figure 4.13: Comparison to literature on the redshift evolution of the faint end slope, $\alpha$, and the characteristic magnitude, $m^{*}$. 

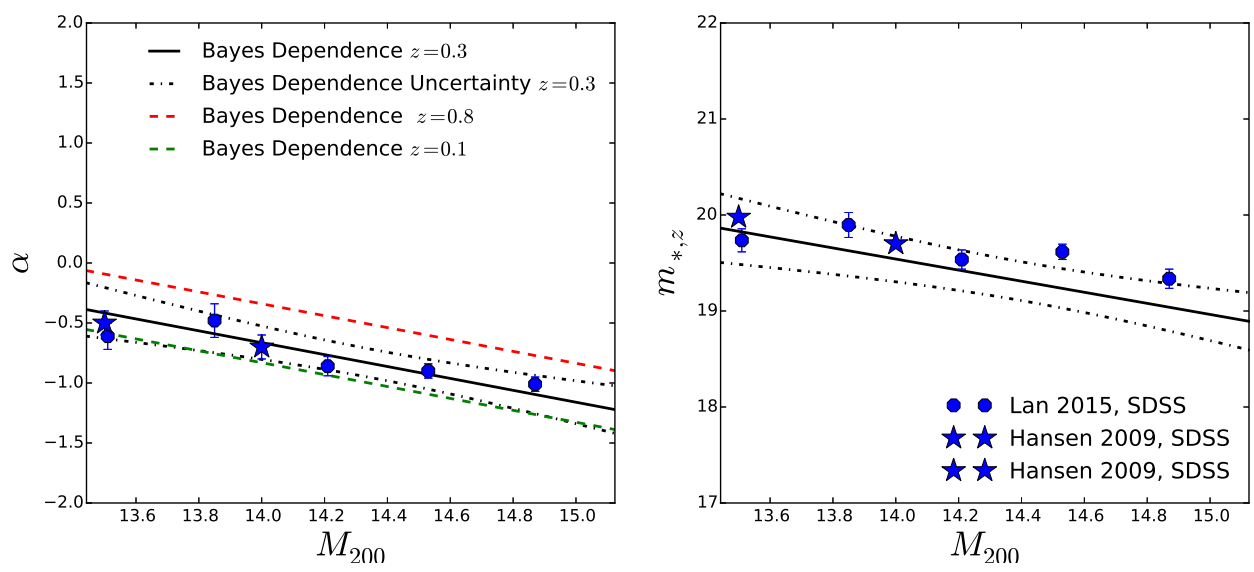

Figure 4.14: Comparison to literature on the mass dependence of the faint end slope, $\alpha$, and the characteristic magnitude, $m^{*}$.

\subsection{Discussion}

\subsubsection{Comparison to Literature}

In Figure 4.13 and Figure 4.14, we compare our results to literature measurements.

At low redshift, literature constraints are available from SDSS data in Hansen et al. (2009, $z \sim 0.25$ ) and Lan et al. (2015, $z<0.05)$. These SDSS constraints are modeled to be mass dependent. In Figure 4.13, we show our constraints derived for the $\log M_{200} \sim 14.5$ mass range. The SDSS faint end slope measurements, $\alpha$, appear to be $\sim 1.0 \sigma$ shallower than our result at the low redshift end. Meanwhile, the characteristic magnitude measurements, $m_{z}^{*}$, also appears to be $\sim 1.0 \sigma$ fainter than our result. Because $\alpha$ and $m_{z}^{*}$ measurements are correlated, the discrepancy can be caused by either $\alpha$ or $m_{z}^{*}$. In addition, the SDSS results are derived with $r$ (Lan et al., 2015, $z<0.05$ ) or $i$ (Lan et al., 2015, $z<0.05$ ) band data. Using $i$-band luminosity for the lower redshift range helps diminishing the discrepancy, but the results are not significantly different from using $z$-band luminosity. A larger sample is needed to better understand the difference.

In figure 4.14, the $\alpha$ measurements from Lan et al. (2015, $z<0.05)$ in different mass bins match well with our results. The mass dependent constraints for red sequence galaxies in Hansen et al. (2009) are not explicitly listed, but there is a clear trend of $\alpha$ steepening in the mass range of $\left[10^{13} \mathrm{M}_{\odot}, 10^{14} \mathrm{M}_{\odot}\right]$, and then stabilizing beyond $10^{14} \mathrm{M}_{\odot}$. The measurements qualitatively agrees with our result.

However, our constraint of $\alpha$ appears to be shallower than the measurements in Yang et al. (2009). This is possibly caused by the different magnitude limits of the two studies. Our measurement performs a single Schechter function to only the bright part of the lumi- 
nosity function ( $m<m_{*}+2$ mag), about $\sim 1$ mag shallower than the Yang et al. (2009) measurement. At lower luminosity, a single Schechter function may not provide a best fit to the data, as the luminosity function displays a turn-up feature (Trentham \& Hodgkin, 2002; Popesso et al., 2006; Barkhouse et al., 2007; Bañados et al., 2010; Yamanoi et al., 2012), mostly beyond $m_{*}+3$ mag (Lan et al., 2015).

At intermediate to high redshift, measurements of cluster red sequence luminosity function are still scarce. The literature constraints we show in Figure 4.13 are either based on a handful of clusters or are based on individual clusters. Note that the mass dependence of $\alpha$ makes it difficult to make a direct comparison in Figure 4.13. It is important to consider the cluster mass range when making comparison between our measurements and literature values. For example, De Propris et al. (2013) measures steeper $\alpha$, but the sample used in that study contains massive clusters.

The correlation between $m^{*}$ and cluster mass qualitatively agrees with the measurements in Lan et al. (2015, $z_{i}$ 0.05) from SDSS data. The SDSS $m^{*}$ measurement for red sequence galaxies at higher redshifts in Hansen et al. (2009, $z>\sim 0.25$ ) are not explicitly listed, but there is also a trend of brightening in the mass range of $\left[10^{13} \mathrm{M}_{\odot}, 5 \times 10^{14} \mathrm{M}_{\odot}\right]$, and then stabilizing beyond $5 \times 10^{14} \mathrm{M}_{\odot}$.

\subsubsection{The Methods}

To understand the accuracies on constraining the RSLF $\alpha$ and $m^{*}$ parameters, we simulate cluster galaxy luminosity distribution by sampling from a Schechter function distribution. We also generate field galaxies with an exponential magnitude distribution, and mix the simulated cluster galaxies and the simulated field galaxies. The number ratio between cluster galaxies and field galaxies is approximately 1.0 to $m^{*}+2$ mag, and the total number of cluster galaxies is approximately 350 (matching the typical numbers in one redshift bin/mass bin discussed in Section 4.6.1.

We apply the basic Bayes model (no need to use Bayes dependence model because $\alpha$ and $m_{*}$ are fixed) to the simulated data. The background distribution is estimated with a larger simulated background sample. In Figure 4.15, we show the differences between the derived $\alpha$ or $m_{*}$ and their truth values. The fitting was performed to different magnitude limit from $m_{*}+0.5 \mathrm{mag}$ to $m_{*}+4.5 \mathrm{mag}$. We find that fitting to a magnitude limit below $m_{*}+2.0$ mag tend to result in bias (Figure 4.15), although the direction of the biases change with the background galaxy distribution.

We have also tried applying the Histogram method to the simulated data. The conclusions are similar, but the results appear to be less robust compared to the Bayesian method. 

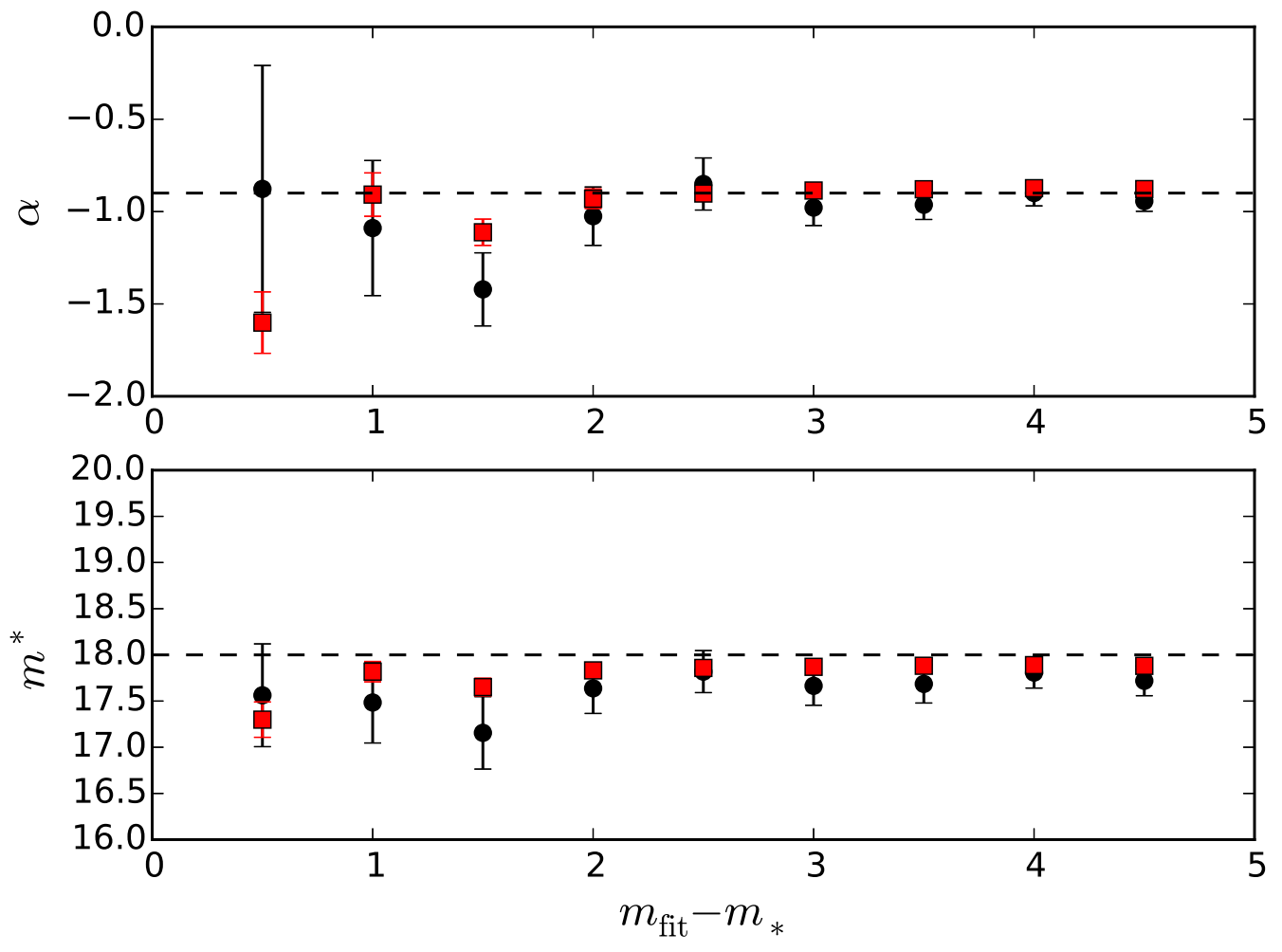

Figure 4.15: Simulation test of the Bayes method. The dashed lines show the truth input values of $\alpha$ and $m_{*}$. The black filled circles and red filled squares show the constraints derived with samples that match the sizes of our current data and future data release respectively. 
Finally, we expect XCS to find over 1000 clusters with future full DES data. On the other hand, optical cluster finders (Rykoff et al. in prep.) has already identified $\sim 1000$ clusters in DES data. Enlarging the sample size of the simulated galaxies by 10 times, we expect to see sensitivity improvement by $\sim 3$ times (red squares in Figure 4.15).

\subsubsection{Implication on Cluster Red Sequence Formation}

In this work, we provide evidence for mild redshift evolution of the faint end slope. The relative abundance of faint red sequence galaxies up to $m^{*}+2$ mag appears to have built up with time. However, since $\alpha$ is low in the redshift range of $[0.5,1.0]$, many of the faint red sequence galaxies must have already been in place.

Since the low mass field galaxy $\left(m^{*}+3 \mathrm{mag}\right)$ population contains significantly higher fraction of blue galaxies (e.g., Peng et al., 2010b; Hoyle et al., 2012; Zhang et al., 2013), the mild redshift evolution of $\alpha$ favors a processing scenario that many of the faint galaxies have already been transitioned to red before completing the cluster infall process. Still, the abundance of faint red sequence galaxies appears to increase mildly with time, which indicates that the transition from blue to red is not immediate. Galaxy clusters would only contain red sequence galaxies, and also many faint red sequence galaxies if the transition was immediate. This analysis is further supported by the observation of an increasing fraction of red sequence galaxies in literature (Butcher-Oemler Effect: Butcher \& Oemler, 1984). On the other hand, the transition time from blue to red for faint cluster galaxies can not last for long comparing to the $z=1.0$ to $z=0.2$ time span, as we would notice a stronger redshift evolution of $\alpha$ in that scenario. Combining the evolution of the faint end slope together with cluster accretion history in simulation may help constraining the transition time of red sequence galaxies (McGee et al., 2009).

Furthermore, we notice hints that $\alpha$ may be dependent on cluster mass, that more massive clusters are more abundant in terms of faint red sequence galaxies. It indicates a better evolved red sequence population in massive clusters. This mass dependence effect may provide one clue toward explaining the discrepancies in constraining the RSLF redshift evolution of previous studies. Examine the redshift evolution of RSLF may require comparing clusters in a similar mass range.

The characteristic magnitude also appears to change for clusters of different masses. More massive clusters appear to have brighter characteristic magnitude. This can be explained with the scenario that as clusters grow through merging and galaxy infall, cluster galaxies also grow in mass and size (Laporte et al., 2013). 


\subsection{Summary}

In this Chapter, we conduct a statistical analysis of the evolution of the cluster galaxy (or red sequence) luminosity function using Bayesian modeling methods. Specifically, we develop a Bayesian model that can simultaneously constrain the redshift evolution and the cluster mass dependence of red-sequence luminosity function. The model incorporates the mass uncertainties of the clusters.

We find weak statistical evidence of both mild redshift evolution and mass dependence of the Schechter function faint-end slope. We also confirm the previously known cluster mass dependence of the characteristic magnitude. These conclusions are detected at a significance level of $2 \sigma$.

The mass dependence of RSLF parameters may explain the different conclusions of previous studies that model RSLF redshift evolution. The redshift evolution and mass dependence of RSLF parameters will need to be further investigated with larger cluster samples that cover the redshift range from 0 to 1.0. Future XCS-DES data release, or DES optical cluster samples should allow us to improve the sensitivity of the analysis by $\sim 3$ times. 


\section{CHAPTER 5}

\section{Cluster Ambience - Galaxy Filaments}

\subsection{Topic Introduction}

In the standard picture of structure formation after the Big Bang, initial density perturbations grow via gravitational instability and form massive structures in a bottom-up, hierarchical growth scenario in which small, gravitationally bound structure like galaxies conglomerate into progressively larger structures like galaxy groups and clusters. On the largest scales, gravitational instability leads to a collapse of matter first into sheets (Zeldovich, 1970) and then into a network of filaments (Klypin \& Shandarin, 1983) with galaxy clusters at the intersection of filaments. This early result of Klypin \& Shandarin has been confirmed in countless $N$-body simulations since then (e.g., Davis et al., 1985; Bertschinger \& Gelb, 1991; Springel et al., 2005).

Observationally the filamentary large-scale structure (LSS) has been traced by the galaxy distribution in redshift surveys for decades; from first indications seen by Jõeveer et al. (1978) over the seminal work of Geller \& Huchra (1989) to modern redshift surveys like 2dF (Colless et al., 2001) and SDSS (Ahn et al., 2012). The gaseous WarmHot Intergalactic Medium residing in filaments was seen in X-ray emission (Werner et al., 2008; Fraser-McKelvie et al., 2011) and absorption (Buote et al., 2009; Fang et al., 2010). Malarecki et al. (2013) use the lobes of giant radio galaxies in an attempt to probe the physical condition of the WHIM below the densities accessible by X-ray observations. It is, however, not clear that assumption they make on the state of the gas in radio lobes (equipartition and minimum Lorentz factor) as well as the assumption on pressure equilibrium of the radio lobes with the surrounding WHIM are fully justified. More recently the underlying Dark Matter skeleton of large-scale structure filaments was also detected through its gravitational lensing effect (Dietrich et al., 2012; Jauzac et al., 2012).

Despite these observational advances, filaments remain relatively little studied, yet they are astrophysically interesting places: LSS filaments contain a plurality of all matter in the 
Universe (Aragón-Calvo et al., 2010) and they harbor the "missing baryons" at low redshift (Davé et al., 2001). It is well known that galaxies in clusters are redder than galaxies in the surrounding field and mostly have ceased star-formation. As galaxies are constantly accreted into clusters along filaments, it is only reasonable to assume that a portion of the transformation from actively star-forming galaxies to passively evolving ones happens in filaments. The specific role of the filamentary environment in this process has not been studied very much. An exception is, e.g., the work of Porter et al. (2008), which found a spike in star-formation activity in filament galaxies at fixed cluster centric distance. It is of course well established that the local density of galaxies is one of the factors deciding the efficiency with which star-formation is quenched (Peng et al., 2010b). The exact role of the filament versus the galaxy-group environment, however, has not been studied in detail.

The relative dearth of papers about filaments compared to, e.g., the number of studies done of galaxy clusters is due to the low-density contrast of filaments with respect to the mean density of the Universe. While galaxy clusters by definition have a density contrast $\delta>200$, the typical density contrast of filaments is $\delta<20$ (Aragón-Calvo et al., 2010). This makes filaments much more difficult to find, observe, and study, and they have generally been traceable in spectroscopic surveys only to redshift $\sim 0.2$.

In this chapter, we develop an algorithm to study the photometric properties of intercluster galaxy filaments from redshift 0.1 to 0.4 . Because close pairs of galaxy clusters are generally connected by filaments (Pimbblet et al., 2004; Colberg et al., 2005), instead of trying to detect single filaments, our approach is to identify galaxy cluster pairs and then stack the galaxy population between them. By comparing the stack of such populations to the stack of other selected galaxy fields, we can detect an overdensity of galaxies present in the connecting filaments. We go on to analyze the color and luminosity properties of these filament galaxies and to examine these for possible redshift evolution.

The remainder of this chapter is arranged as follows. In Section 5.2, we describe our algorithm and some tests we have conducted on it, and represent the statistical significance of our filament overdensity. Section 5.3 contains our results. We compute color evolution and luminosity function of filament galaxies, as well as their spatial and richness dependence. In Section 5.4, similar results from N-body simulation are provided for comparison. We summarize and discuss the implications of our results in Section 5.5. Throughout this chapter, we assume a flat cosmology with $\Omega_{\mathrm{m}}=0.30$ and $h=0.7$. 


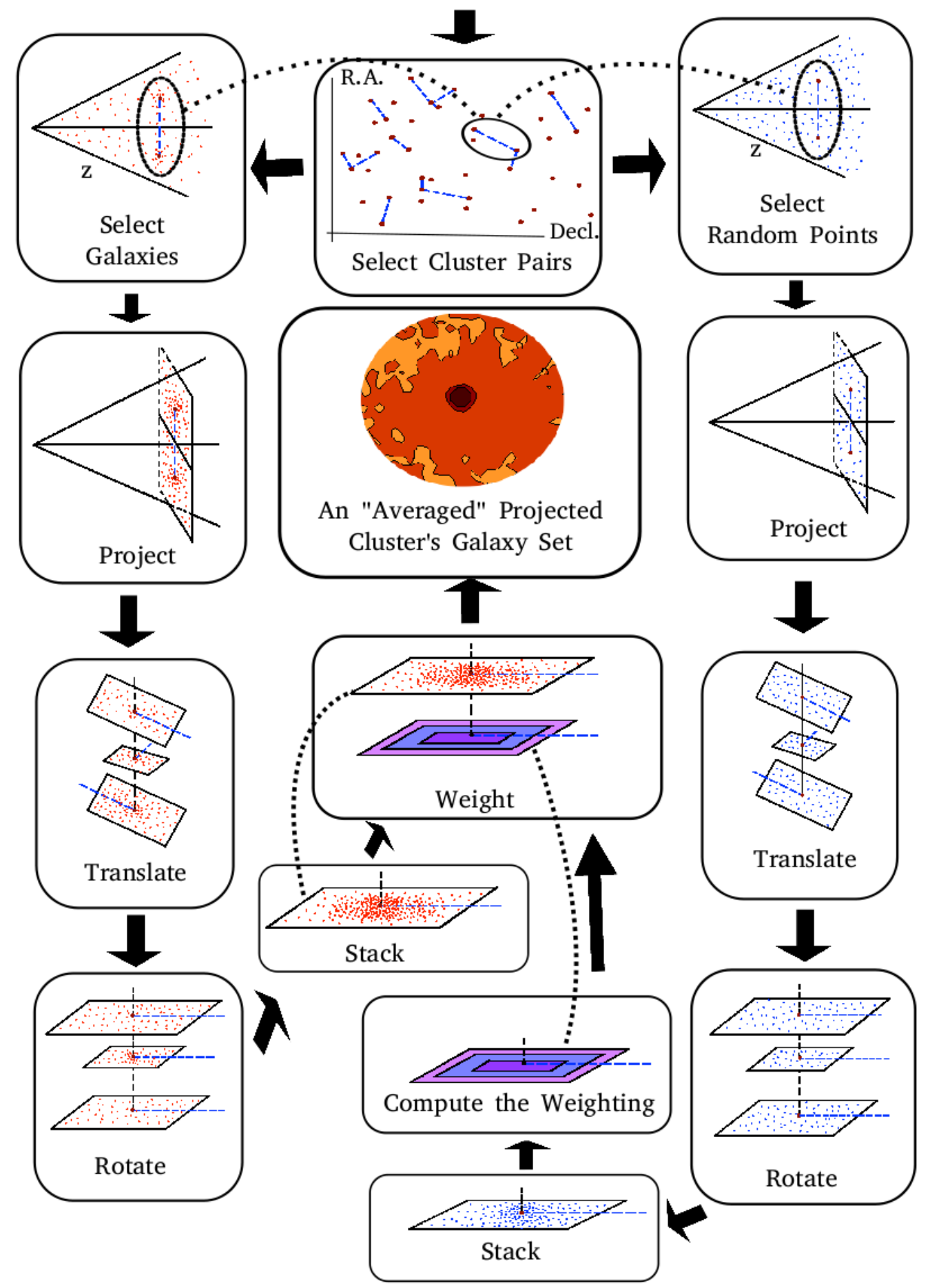

Figure 5.1: Flowchart illustration of the algorithm described in Section 5.2.2. The left side of the figure illustrates the manipulations on galaxy data in order to acquire stacked cluster pair galaxy fields. The right side illustrates the the manipulations on the random point catalog in order to acquire the sky coverage weighting, which tells how many times each pixel in the galaxy stacking is covered by real sky data. The manipulations on random points are identical to the manipulations on real galaxy data before "Compute the Weighting" step (See Section 5.2.2.5 for details). Arrows indicate the processing flow. 


\subsection{Methods}

\subsubsection{Data}

For this study, we use the gmBCG galaxy cluster catalog (Hao et al., 2010). This is a large optically selected galaxy cluster catalog constructed from the SDSS Data Release 7 (Abazajian et al., 2009). It includes 55,424 clusters in the redshift range $0.1<z<$ 0.55 , covering the whole SDSS Data Release 7 area.When building this catalog, Hao et al. searched for overdensities of red sequence galaxies around brightest cluster galaxy (BCG) candidates. The final catalog contains positions, estimated redshifts, and richness for each detected cluster. Comparing to other previously well accepted cluster catalogs (Miller et al., 2005; Koester et al., 2007a) in the SDSS footprint, this catalog has larger sky coverage, extends to deeper redshift, and has been extensively tested.

The galaxy catalog we use for this study is constructed from SDSS DR8 BOSS imaging data (Eisenstein et al., 2011; Aihara et al., 2011). The sky coverage of this catalog is described by a catalog of random points, which samples the same angular coverage as the survey objects. Instead of using SDSS DR7, we choose to use galaxy data from the BOSS survey in a newer SDSS data release because of its improved photometry. This choice leaves the galaxy catalog having a slightly different sky coverage with the gmBCG cluster catalog. However, with the sky coverage weighting described in Section 5.2.2.5, only the overlapping area of the galaxy catalog and the cluster catalog contributes to our final results.

\subsubsection{Algorithm}

Figure 5.1 outlines our algorithm, which is described in detail in this section. To summarize, we select pairs of galaxy clusters and identify the galaxies which occupy the regions around these cluster pairs. We stack these galaxies, weight for sky coverage, and compare the galaxy population between cluster pairs to the galaxy population seen in other fields.

To test our methods and compare to our SDSS results, we also apply our algorithm to the Millennium simulation (Springel et al., 2005). The procedures and results of this application are discussed in the appendix.

\subsubsection{Cluster Pair Selection}

We begin by selecting the cluster pairs to be used in our algorithm. According to Colberg et al. (2005), the probability of finding a filament between galaxy clusters is strongly dependent on their separation. Clusters separated by less than $5 h^{-1} \mathrm{Mpc}$ always have a connection between them, but this is primarily formed from the outer regions of the cluster 


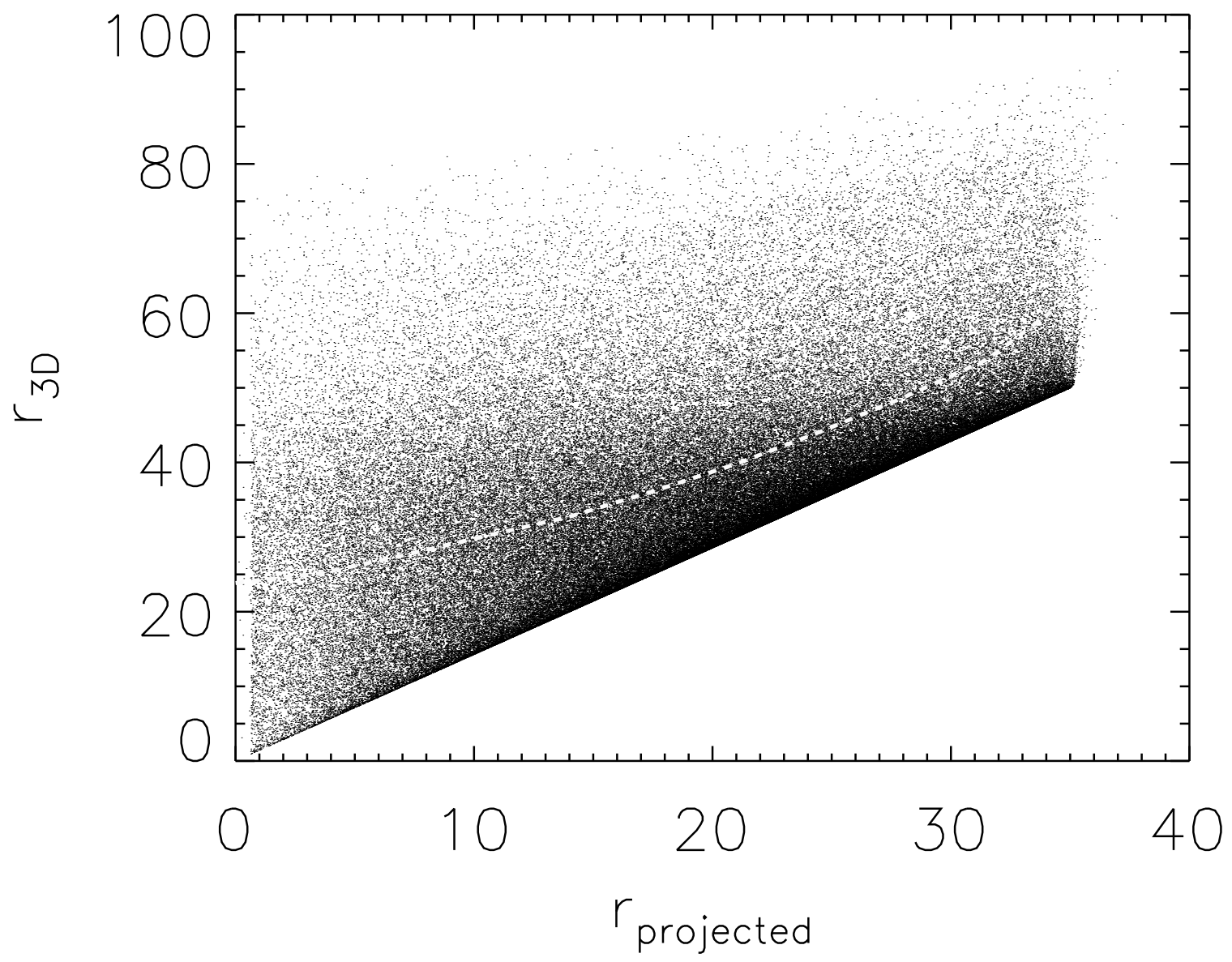

Figure 5.2: Cluster pairs' projected separation on the plane of the sky at their median redshift, $r_{\text {projected, }}$, versus their 3-d physical separation, $r_{3 \mathrm{D}}$. The white dashed line shows the mean $r_{3 \mathrm{D}}$ value at different $r_{\text {projected. }}$. We assume the clusters' photometric redshift are purely due to Hubble flow and use the law of cosine for calculating $r_{3 \mathrm{D}}$. The cluster pairs' projected separation on the plane of the sky is cut off at $\sim 35 h^{-1} \mathrm{Mpc}$, depending on the exact redshift of the projection, but their 3-d separation can be as large as $90 h^{-1} \mathrm{Mpc}$. 
populations rather than a separate filament population. For clusters that are separated between $15 h^{-1} \mathrm{Mpc}$ to $25 h^{-1} \mathrm{Mpc}$, about $1 / 3$ of them have a filament connection. For clusters that are separated less than $35 h^{-1} \mathrm{Mpc}$, the probability of filament existence between them is larger than $10 \%$. Clusters separated more than $50 h^{-1} \mathrm{Mpc}$ are unlikely to have filaments between them. Also, richer clusters tend to have more filaments connecting to them, but the number of connections rarely exceeds 5 . Informed by these expectations from simulation, we select cluster pairs separated by less than $\sim 35 h^{-1} \mathrm{Mpc}$ while limiting the maximum number of cluster pairs one cluster can appear in. For each cluster, the clusters paired to it satisfy the following conditions,

1. The difference in photometric redshift of the BCGs of the two clusters should differ by $<0.02$. This redshift difference roughly corresponds to a comoving distance of $50 h^{-1} \mathrm{Mpc}$.

2. The angular separation between the two clusters is smaller than $35 h^{-1} \mathrm{Mpc}$ on the plane of the sky at the redshift of the the cluster to-be-paired-to. Because we do not directly constrain the two clusters' 3-d physical separation, the cluster pairs' 3-d physical separation can be larger than $35 h^{-1} \mathrm{Mpc}$, as shown in Figure 5.2.

3. Each cluster can appear in several cluster pairs if it has more than one neighbor meeting the above criteria. If a cluster appears in more than 5 cluster pairs, we keep its 5 closest pairs along with all pairs that include clusters with fewer than 5 neighbors.

With this selection procedure, we identify a total of 160,954 cluster pairs. As discussed in Section 5.2.2.6, when stacking galaxy populations that are around these cluster pairs, we apply an absolute magnitude cut which is above the SDSS completeness limit at $z=$ 0.38 , even with dust extinction correction and K-correction. To avoid including galaxy populations in very dusty sky regions, we exclude cluster pairs which fall within $1^{\circ} \times 1^{\circ}$ angular boxes around galaxy objects which have dust extinction in r-band larger than 0.4. This lowers the number of cluster pairs to 158,897 in the final catalog. In this cluster pair catalog, one cluster on average appears in 5.7 cluster pairs.

\subsubsection{Selection of Galaxies}

Inter-cluster filaments do not always strictly lie on the inter-cluster axis. According to the studies of Pimbblet et al. and Colberg et al., maybe $40 \%$ filaments run straightly from one cluster to the other one, and maybe a similar portion still appearing to be strand-like but 
curves from one cluster to the other one, and the rest being wall or sheet-like, irregular or even permeative with nondefinitive morphologies. In this work, we do not try to distinguish filaments of different morphologies. We try to incorporate all kinds of genuine structures that cause an overdensity of matter between a cluster pair.

For every cluster pair, we identify all galaxies in and around the two clusters. We first identify all galaxies in a rectangular box centered on the filament midpoint, with the width of the inter-cluster distance but twice as long. Galaxies in the square centered on each cluster form the "galaxy set" for that cluster. Each galaxy set includes the cluster, an intercluster region in which we expect to find a filament, and an opposing field region in which we do not (necessarily) expect a filament.

When selecting these neighboring galaxies, we do not make any redshift cuts. It is possible that a carefully designed photometric redshift cut could increase the significance of the results discussed in Section 5.2.3, but such a cut risks to introducing color and magnitude dependent selection effects. While photometric redshifts for bright passively evolving galaxies can be smaller than 0.05 (Cunha et al., 2009), those for fainter or star-forming galaxies can be substantially worse.

\subsubsection{Cluster Galaxy Set Processing}

For each cluster in one cluster pair, there is one galaxy set corresponding to it. We denote the angular coordinates of one cluster in one cluster pair to be $\left(\alpha_{0}, \delta_{0}\right)$, its comoving distance to be $d_{\mathrm{C}}$, the angular coordinates of the $g$ th galaxy in its galaxy set to be $\left(\alpha_{\mathrm{g}}, \delta_{\mathrm{g}}\right)$, and the angular coordinates of the other cluster BCG in this cluster pair to be $\left(\alpha_{0 \mathrm{p}}, \delta_{0 \mathrm{p}}\right)$. Before stacking the galaxy sets of different clusters, we need to redefine galaxy coordinates so that they convey the same physical scale at different redshifts:

1. Project these galaxies onto a tangent plane at the cluster BCG,

$$
\begin{aligned}
& \theta_{\mathrm{g}}=\alpha_{\mathrm{g}} \cos \left(\delta_{0}\right), \\
& \delta_{\mathrm{g}}=\delta_{\mathrm{g}},
\end{aligned}
$$

so that $1^{\circ}$ in the $\theta_{\mathrm{g}}$ and $\delta_{\mathrm{g}}$ dimension corresponds to the same great-circle distance.

2. Translate $\left(\theta_{\mathrm{g}}, \delta_{\mathrm{g}}\right)$ as in

$$
\begin{aligned}
& \theta_{\mathrm{g}}^{\prime}=\theta_{\mathrm{g}}-\left(\alpha_{0} \cos \left(\delta_{0}\right)\right), \\
& \delta_{\mathrm{g}}^{\prime}=\delta_{\mathrm{g}}-\delta_{0},
\end{aligned}
$$

so that the cluster center of the galaxy set is placed at $(0,0)$. 
3. Rotate the galaxy set with a rotation matrix $M$,

$$
\left[\begin{array}{c}
\theta_{\mathrm{g}}^{\prime \prime} \\
\delta_{\mathrm{g}}^{\prime \prime}
\end{array}\right]=M \times\left[\begin{array}{c}
\theta_{\mathrm{g}}^{\prime} \\
\delta_{\mathrm{g}}^{\prime}
\end{array}\right]
$$

to have the inter-cluster axis lay on $y=0$, with the direction extending to the other cluster aligned toward $x>0$. The rotation matrix $M$ has the form of:

$$
M=\left[\begin{array}{cc}
\cos \left(\arctan \frac{\delta_{0 \mathrm{p}}-\delta_{0}}{\left(\alpha_{0 \mathrm{p}}-\alpha_{0}\right) \cos \delta_{0}}\right) & \sin \left(\arctan \frac{\delta_{0 \mathrm{p}}-\delta_{0}}{\left(\alpha_{0 \mathrm{p}}-\alpha_{0}\right) \cos \delta_{0}}\right) \\
-\sin \left(\arctan \frac{\delta_{0 \mathrm{p}}-\delta_{0}}{\left(\alpha_{0 \mathrm{p}}-\alpha_{0}\right) \cos \delta_{0}}\right) & \cos \left(\arctan \frac{\delta_{0 \mathrm{p}}-\delta_{0}}{\left(\alpha_{0 \mathrm{p}}-\alpha_{0}\right) \cos \delta_{0}}\right)
\end{array}\right] .
$$

4. Redefine the coordinates to be

$$
\begin{aligned}
& x=\theta_{\mathrm{g}}^{\prime \prime} \times\left(d_{\mathrm{C}} \tan 1^{\circ}\right), \\
& y=\delta_{\mathrm{g}}^{\prime \prime} \times\left(d_{\mathrm{C}} \tan 1^{\circ}\right) .
\end{aligned}
$$

Here, $d_{\mathrm{C}} \tan 1^{\circ}$ is the physical distance that corresponds to $1^{\circ}$ angular separation on the tangent plane at the cluster center. If all the galaxies in the galaxy set are at about the same redshift with the cluster BCG, the coordinates re-defined above would reflect the projected physical distance between these galaxies to the cluster BCG in the plane of the sky. This argument does not hold for the whole population since we do not make any redshift selection. However, this definition of coordinates should be proper for filament and cluster galaxies after foreground and background subtraction.

\subsubsection{Stacking}

When stacking, we create a multidimensional galaxy stack in bins of cluster pair redshift. Each stack is four dimensional, including galaxy coordinates $(x, y)$, galaxy absolute magnitude $M_{r}$, and the observer frame dust extinction corrected $g-r$ color. The binning size in each dimension is chosen to be small enough not to smear out the details of $x, y, M_{r}$ and $g-r$ dependence, but not so small as to leave many bins unoccupied. For the $x$ and $y$ dimensions, the bin sizes are both $0.7 \mathrm{Mpc}$, because of the low $\mathrm{S} / \mathrm{N}$ in one spatial bin. For binning in $M_{r}$ and $g-r$, the bin sizes are $0.2 \mathrm{mag}$ and $0.125 \mathrm{mag}$. The value of the stack at one particular bin $X_{i-1}<x \leq X_{i}, Y_{j-1}<y \leq Y_{j}, M_{r(l-1)}<M_{r} \leq M_{r l}$ and $(g-r)_{k-1}<(g-r) \leq(g-r)_{k}$ is denoted as $G_{z}\left(x_{i}, y_{j}, M_{r l},(g-r)_{k}\right)$. To include a comparison of cluster galaxies to filament galaxies, we also build a two dimensional stack in $M_{r}$ and $g-r$ with galaxies around cluster centers which satisfy $\sqrt{x^{2}+y^{2}}<0.7 \mathrm{Mpc}$. 
This stack of cluster galaxies is denoted as $C L_{z}\left(M_{r}, g-r\right)$.

\subsubsection{Stack Weighting}

Because the galaxy cluster pairs have different separations, and the galaxy sets of different clusters are selected based on their cluster pair separations, the physical sizes of our cluster galaxy sets vary. Also, the cluster pairs may fall at the edge of the survey, and the galaxy sets will have irregular sky coverage. Without accounting for this, we would observe artificial galaxy density gradients in the $x$ and $y$ dimension of the stacking. To remove such an artificial gradient, we weight $G_{z}\left(x, y, M_{r}, g-r\right)$ with a coverage weighting function $W_{z}(x, y)$. We first explain the physical interpretation of $W_{z}(x, y)$ and the weighting procedure in this and the next paragraph, and describe details on computing $W_{z}(x, y)$ in the rest of this section. The value of $W_{z}(x, y)$ at $X_{i-1}<x \leq X_{i}$ and $Y_{j-1}<y \leq Y_{j}$, denoted as $W_{z}\left(X_{i}, Y_{j}\right)$, tells how many cluster galaxy sets include valid data for the pixel at $X_{i-1}<x \leq X_{i}$ and $Y_{j-1}<y \leq Y_{j}$. For example, $W_{z}\left(X_{i}, Y_{j}\right)=3$ when there are 3 clusters'

galaxy sets fully covering this pixel, or $W_{z}\left(X_{i}, Y_{j}\right)=\frac{10}{3}$ when there are 3 clusters' galaxy sets fully covering this pixel and another cluster's galaxy set covering $1 / 3$ of this pixel.

We weight $G_{z}\left(x, y, M_{r}, g-r\right)$ with Equation 5.6, and acquire a weighted distribution, $g_{z}\left(x, y, M_{r}, g-r\right)$, the value of which at one bin, $X_{i-1}<x \leq X_{i}, Y_{j-1}<y \leq Y_{j}$, $M_{r(l-1)}<M_{r} \leq M_{r l}$ and $(g-r)_{k-1}<(g-r) \leq(g-r)_{k}$, is denoted as $g_{z}\left(x_{i}, y_{j}, M_{r l},(g-\right.$ $\left.r)_{k}\right)$ :

$$
g_{z}\left(x_{i}, y_{j}, M_{r l},(g-r)_{k}\right)=\frac{G_{z}\left(x_{i}, y_{j}, M_{r l},(g-r)_{k}\right)}{W_{z}\left(x_{i}, y_{j}\right)} .
$$

This weighted distribution, $g_{z}\left(x, y, M_{r}, g-r\right)$, can be interpreted as representing properties of one "averaged" cluster's galaxy set with filament connection on the $x>0$ side.

The $W_{z}(x, y)$ we use is built from the random point catalog described in Section 5.2.1. This random point catalog first goes through the same algorithm with the galaxy catalog as described in Section 5.2.2.2 and Section 5.2.2.3, and then is stacked together. Unlike Section 5.2.2.4, in which we build a four dimensional distribution from stacking galaxies, we can only build a two dimensional distribution in $x$ and $y$ from stacking random points, denoted as $R_{z}(x, y)$. At $X_{i-1}<x \leq X_{i}$ and $Y_{j-1}<y \leq Y_{j}$, the value of $R_{z}(x, y)$, denoted as $R_{z}\left(X_{i}, Y_{j}\right)$, tells how many random points there are in this bin. Because the random point catalog covers the survey area evenly, $R_{z}(x, y)$ can be used as a proxy of $W_{z}(x, y)$. The relation between $R_{z}(x, y)$ and $W_{z}(x, y)$ at one particular bin is

$$
R_{z}\left(x_{i}, y_{j}\right)=W_{z}\left(x_{i}, y_{j}\right) \times C(z)
$$


Here $C(z)$ is the average number of random points per $\left[h^{-1} \mathrm{Mpc}\right]^{2}$ at redshift $z$, and $z$ is the median redshift value of the clusters which have their galaxy sets or random point sets stacked to build $G_{z}(x, y, M-r)$ or $R_{z}(x, y)$. $C(z)$ can be computed from the comoving distance, denoted by $d_{\mathrm{C}}(z)$, at redshift $z$, and the average number of random points per $\operatorname{deg}^{2}$, denoted by $C$, through

$$
C(z)=C \times\left[\arctan \left(\frac{0.7 h^{-1} \mathrm{Mpc}}{d_{\mathrm{C}}(z)}\right)\right]^{2} .
$$

With known values of $R_{z}(x, y)$, and $C$, We compute $W_{z}(x, y)$ from Equation 5.7 and Equation 5.8, and then $g_{z}\left(x, y, M_{r}, g-r\right)$ from Equation 5.6, which is used for deriving the scientific results in this chapter.

To weight $C L_{z}\left(M_{r}, g-r\right)$, which is used for comparison with $g_{z}\left(x, y, M_{r}, g-r\right)$, we record the total number of random points that fall around cluster centers with $\sqrt{x^{2}+y^{2}}<$ $0.7 h^{-1} \mathrm{Mpc}$, denoted by $R_{z}$, and compute the weighted function of $C L_{z}\left(M_{r}, g-r\right)$, which is denoted by $c l_{z}\left(M_{r}, g-r\right)$, with following equations:

$$
\begin{aligned}
R_{z} & =W_{z} \times C(z) \\
c l_{z}\left(M_{r l},(g-r)_{k}\right) & =\frac{C L_{z}\left(M_{r l},(g-r)_{k}\right)}{W_{z}} .
\end{aligned}
$$

In this weighting procedure, one might worry that the poisson noise of the random point sampling would lower the $\mathrm{S} / \mathrm{N}$ of $g\left(x, y, M_{r}, g-r\right)$ or $c l_{z}\left(M_{r}, g-r\right)$. Here, we show that with proper random point density, this influence is insignificant. The poisson noise in $R_{z}(x, y)$ and $R_{z}$ increases with redshifts because $C(z)$ decreases with redshifts. Our random point catalog samples the survey coverage with $C=9,275$ and at $z=0.40$, $C(z)=5.98$. Since we always stack galaxy sets of more than 5,000 clusters in each redshift bin, the $\mathrm{S} / \mathrm{N}$ of $R_{z}(x, y)$ at most spatial bins, and of $R_{z}$, is larger than 100 . At this significance level, the weighting constructed from $R_{z}(x, y)$ and $R_{z}$ brings in negligible noise to $g\left(x, y, M_{r}, g-r\right)$ or $c l_{z}\left(M_{r}, g-r\right)$.

\subsubsection{Galaxy Absolute Magnitude and Luminosity Cut}

For every galaxy selected in Section 5.2.2.2, we compute an absolute magnitude from its apparent magnitude, $m_{r}$, SDSS dust extinction correction, $e$, K-correction, $K$, and its clus- 
ter BCG's luminosity distance $d_{\mathrm{L}}$, with equation

$$
M_{r}=m_{r}-5 \log \left(\frac{d_{\mathrm{L}}}{10 \mathrm{pc}}\right)-e-K .
$$

We compute the K-correction for each galaxy with analytical approximations provided by Chilingarian et al. (2010), since the more popular SED template fitting method is inefficient to implement with our large volume of data. This Chilingarian et al. approach approximates K-corrections computed by Fioc \& Rocca-Volmerange (1997) and Blanton \& Roweis (2007) with analytical polynomials of two parameters: redshift and one observed color, and the residual between values measured by this approach and others (Fioc \& Rocca-Volmerange, 1997; Blanton \& Roweis, 2007; Roche et al., 2009) is close to zero for r-band in the redshift range [0.1,0.45] (Chilingarian et al., 2010). In our application of this method, we use each galaxies own $g-r$ color and the cluster BCG's photometric redshift.

Because SDSS is an apparent magnitude limited survey (Stoughton et al., 2002), its imaging data contains different galaxy populations at different redshifts: at lower redshift, the galaxy catalog is more complete, including more intrinsically faint galaxies than at higher redshift. To ensure we are selecting the same galaxy population for comparison in different redshift bins, we apply a $-24.2<M_{r}<-20.4$ cut in Section 5.2.3, Section 5.2.5, Section 5.3.1, Section 5.3.3 and Section 5.3.4. Under this magnitude cut, even with a maximum dust extinction correction, $e_{\max }=0.4$, and a maximum K-correction $K_{\max }=$ 0.6 in the galaxy catalog, a galaxy's absolute magnitude is still above the $95 \%$ completeness limit of r-band at redshift $z=0.38$. In Section 5.3.2, the $M_{r}$ cut is different for different redshift bins, which is listed in Table 5.1. This cut ensures that the galaxy population is more than $95 \%$ complete in each redshift bin.

\subsubsection{Foreground/Background Subtraction and Filament Detection Significance}

In this section, we describe our foreground and background galaxy subtraction and quantify the detection significance of our filament signal. Within the weighted average cluster galaxy set $g_{z}\left(x, y, M_{r}, g-r\right)$ we define a "filament" region (denoted FL), a "cluster" region (denoted CL), and two comparison regions $\mathrm{C} 1$ and $\mathrm{C} 2$. All of these regions are displayed in Figure 5.3. We search for filament galaxies by subtracting the galaxy population observed in $\mathrm{C} 1$ or $\mathrm{C} 2$ from that seen in FL.

Since the filament fields are placed on the right side of the galaxy sets, the "filament" 
(a)

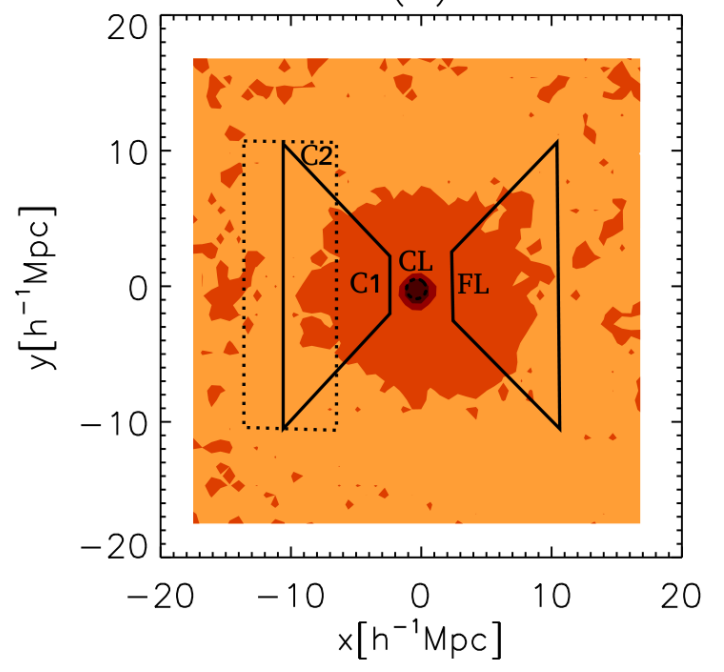

(b)

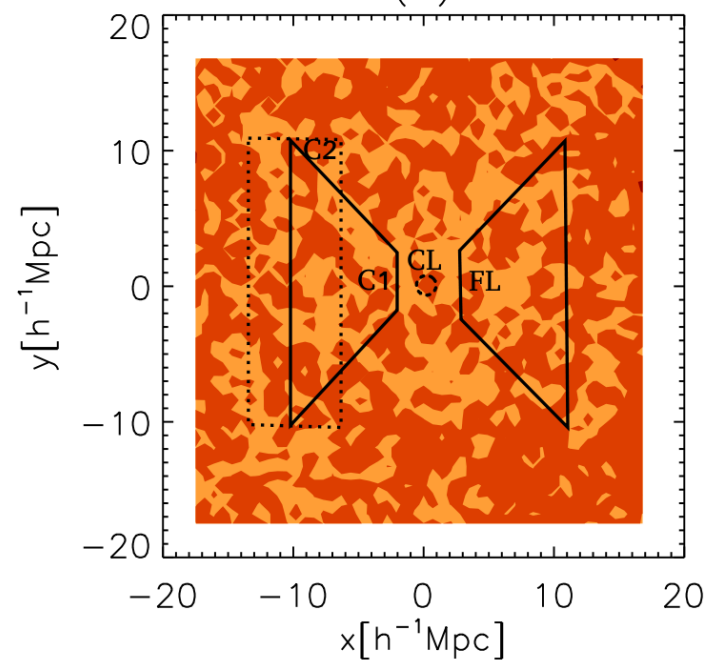

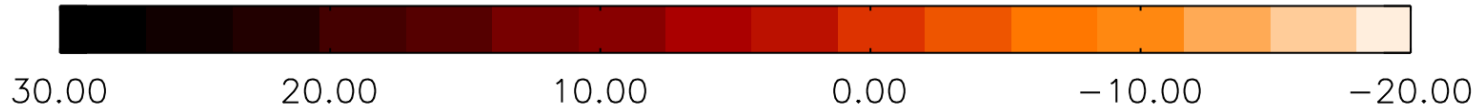

Figure 5.3: (a) Shows a galaxy overdensity significance contour map from stacking galaxy sets of clusters at $0.14<z<0.18$. There is a significant overdensity in the middle of the map, caused by the presence of many cluster galaxies. (b) Shows null test results from stacking randomly re-positioned pairs, where the whole field is noisily flat. In (a) and (b), the solid line and dashed line boxes mark out the four regions defined in Section 5.2.3. 


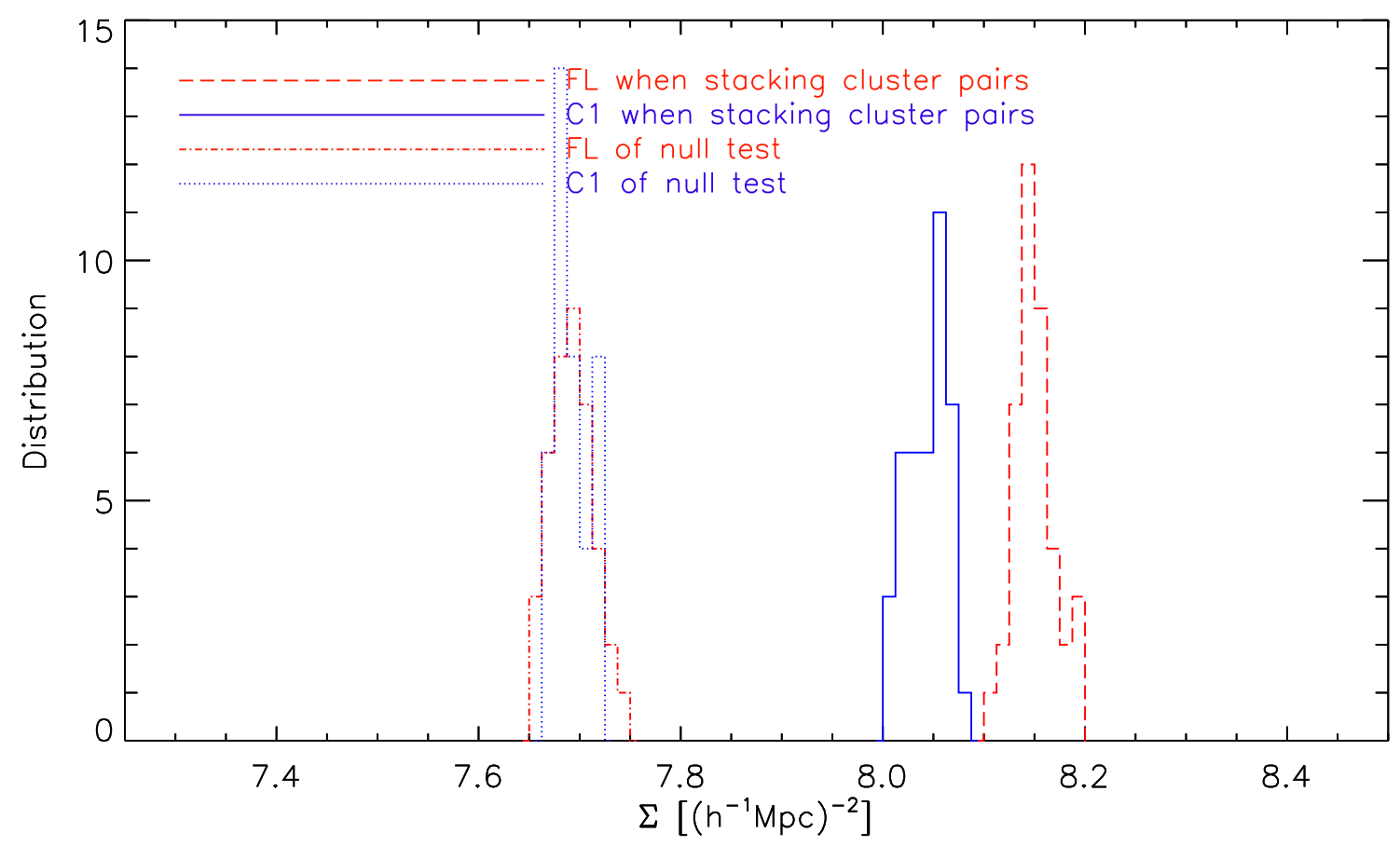

Figure 5.4: Projected galaxy number density in the filament region (FL) and the comparison 1 region $(\mathrm{C} 1)$ at $0.14<z<0.18$ when stacking real cluster pairs or when performing null test with randomly re-positioned pairs (see Section 5.2.5 for details). The histograms shows distributions of 40 bootstrapped resamplings of the original stacking. When stacking galaxy sets of real cluster pairs, the FL region (red dashed line) displays a galaxy overdensity above the $\mathrm{C} 1$ region (blue solid line) with a significance of $\sim 5 \sigma$. Galaxy number counts of FL and $\mathrm{C} 1$ at other redshift slices also show similar high significance detection. In null test of stacking randomly re-positioned pairs, we don't observe any overdensity in F1 (blue dotted line) over $\mathrm{C} 1$ (red dash dot line). Because the galaxy overdensity in cluster fields, the galaxy count in $\mathrm{F} 1$ and $\mathrm{C} 1$ from stacking real cluster pairs are higher than stacking randomly re-positioned pairs. 

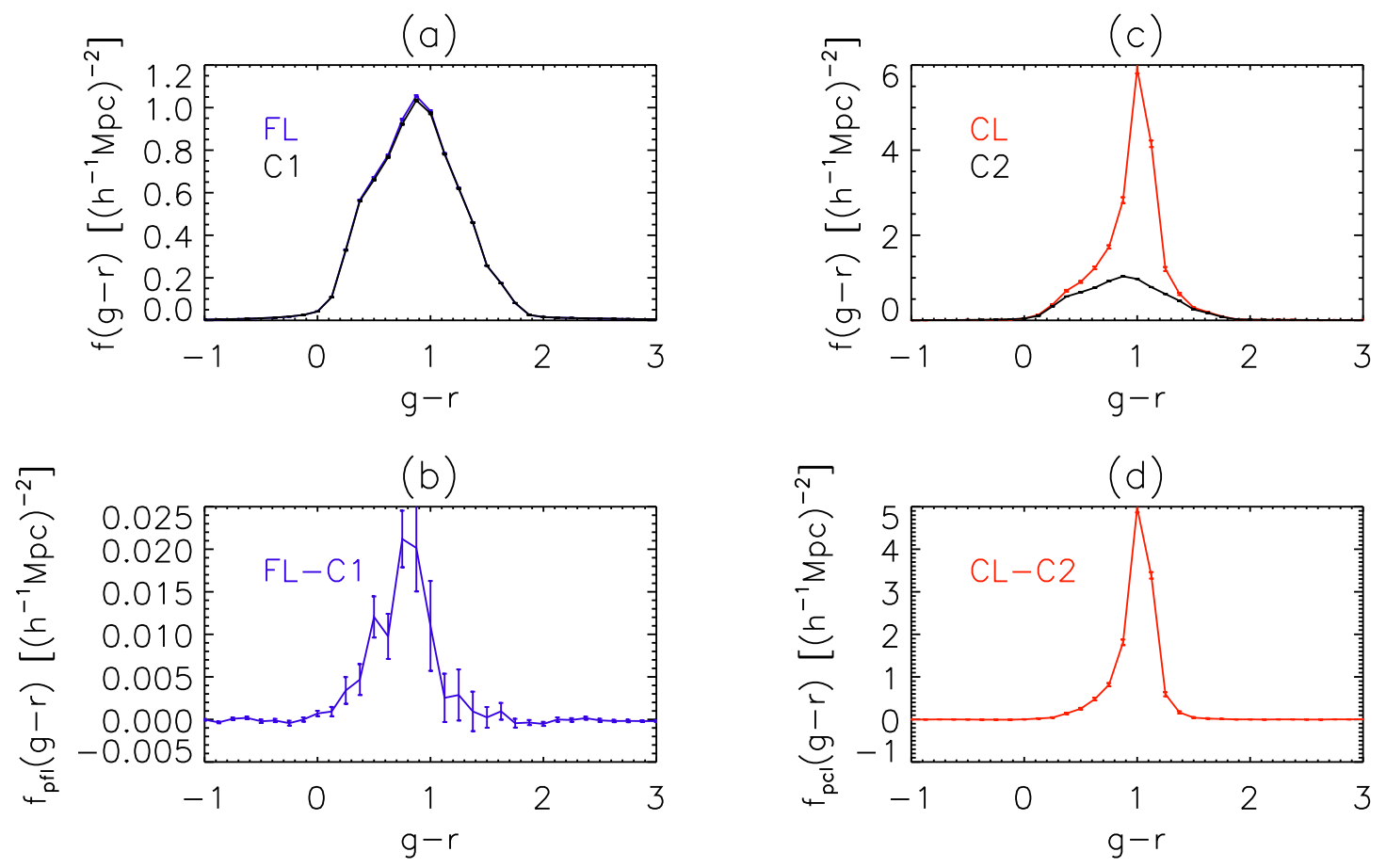

Figure 5.5: How we get the "pure" color distributions of filament galaxies and cluster galaxies. (a) shows the color distributions of the FL region and the $\mathrm{C} 1$ region, which are almost indistinguishable. After subtracting the $\mathrm{C} 1$ region distribution from the FL region distribution, we obtain the color distribution of pure filament galaxies, which is shown in (b). Subtraction between the CL region and the C2 region is also applied to get the pure color distribution of cluster galaxies. (c) shows the color distributions of the CL region galaxies and the $\mathrm{C} 2$ region, and (d) shows the pure color distribution of cluster galaxies after subtraction. These plots are based on stacking galaxy sets of clusters in the redshift bin $[0.14,0.18]$. Unless otherwise noted, the error bars in this figure and the rest of the chapter are single standard deviation errors estimated from bootstrapping the galaxy sets being stacked. Because one cluster on average appears 5.7 times in the stack $(158,897$ cluster pairs versus 55,424 clusters), and the error for clusters tend to be underestimated, we enlarge the error estimation of clusters by a factor of $\sqrt{5.7}$ through out the chapter. 
region is defined with the following constraints:

$$
\begin{gathered}
2.1 \mathrm{Mpc}<x<10.5 \mathrm{Mpc} \\
|\theta=\arctan (y / x)| \leq 45^{\circ} .
\end{gathered}
$$

We define this region to be $2.1 \mathrm{Mpc}$ away from the cluster center to avoid including contamination by cluster galaxies. Although cluster galaxy contents can be removed with the foreground/background subtraction procedure as described later in this section, contamination from this population would lower the $\mathrm{S} / \mathrm{N}$ of filament signals. We also eliminate galaxies that are too far away from cluster centers (with $x>10.5 h^{-1} \mathrm{Mpc}$ ), to avoid lowering the $S / N$ of filament detections with too noisy spatial bins. Since few cluster pairs extend to $10.5 h^{-1} \mathrm{Mpc}$, at the spatial bins beyond this distance, not only the galaxy count noise becomes significant, the random point count noise also starts to influence, resulting in exceptionally low $S / N$ of these bins. The filament region angular extent $|\theta|=45^{\circ}$ is chosen based on discussion in Section 5.3.4. Defining the cluster region is more straightforward. Since cluster BCGs are placed at $(x, y)=(0,0)$, we define the region enclosed within $\sqrt{x^{2}+y^{2}}<0.7 h^{-1} \mathrm{Mpc}$ to be the cluster region, which is also the coverage of functions $C L_{z}\left(M_{r}, g-r\right)$ and $c l_{z}\left(M_{r}, g-r\right)$.

To evaluate signals from the foreground/background galaxy population, we define two comparison regions. The first, $\mathrm{C} 1$, satisfies these constraints:

$$
\begin{aligned}
& -10.5 h^{-1} \mathrm{Mpc}<x<-2.1 h^{-1} \mathrm{Mpc} \\
& |\theta=\arctan (y / x)| \leq 45^{\circ},
\end{aligned}
$$

The second comparison region, C2, satisfies,

$$
\begin{aligned}
& -13.3 h^{-1} \mathrm{Mpc}<x<-6.3 h^{-1} \mathrm{Mpc} \\
& -10.5 h^{-1} \mathrm{Mpc}<y<10.5 h^{-1} \mathrm{Mpc} .
\end{aligned}
$$

The $\mathrm{C} 1$ region is symmetrical to the $\mathrm{FL}$ region, and is used for filament foreground/background subtraction. Both FL and $\mathrm{C} 1$ contain galaxies present in the outskirts of the clusters, so using $\mathrm{C} 1$ for foreground/background subtraction should, to first order, eliminate these. The $\mathrm{C} 2$ region is used for foreground/background signal evaluation in estimating the cluster population. It is picked to be a region away from the cluster center.

In Figure 5.4, we resample with replacement, i.e. bootstrap, cluster pairs which have their galaxy sets stacked, and show the projected galaxy number density in the FL region and the $\mathrm{C} 1$ region. The averaged galaxy number density in the filament region is 
$\sim 5 \sigma$ higher than that of the $\mathrm{C} 1$ region. Since cluster outskirt galaxies have already been taken into consideration when making comparison, this galaxy overdensity is unlikely to be caused by the existence of one galaxy cluster alone, but related to cluster pair structures exclusively. We associate this galaxy overdensity with the filament population between cluster pairs. Such a galaxy overdensity is very small comparing to galaxy overdensity caused by cluster fields. In Figure 5.3(a), we show projected galaxy overdensity significance contour map of stacked galaxy sets at $0.14<z<0.18$. At $\left(X_{i}, Y_{j}\right)$, the projected galaxy overdensity significance, denoted by $S\left(X_{i}, Y_{j}\right)$, is defined as,

$$
S_{z}\left(X_{i}, Y_{j}\right)=\frac{\sum_{k, l} g_{z}\left(X_{i}, Y_{j},\left(M_{r}\right)_{k},(g-r)_{l}\right)-\mu_{z}}{\sigma_{z}} .
$$

Here, $\mu_{z}$ and $\sigma_{z}$ are the mean and scatter of projected galaxy count per spatial pixel in the $\mathrm{C} 2$ region, computed with,

$$
\begin{aligned}
\mu_{z} & =\frac{\sum_{(i, j) \text { in } \mathrm{C} 2} \sum_{k, l} g_{z}\left(X_{i}, Y_{j},\left(M_{r}\right)_{k},(g-r)_{l}\right)}{\sum_{(i, j) \text { in } \mathrm{C} 2}}, \\
\sigma_{z} & =\sqrt{\frac{\sum_{(i, j) \text { in } \mathrm{C} 2}\left[\sum_{k, l} g_{z}\left(X_{i}, Y_{j},\left(M_{r}\right)_{k},(g-r)_{l}\right)-\mu_{z}\right]^{2}}{\sum_{(i, j) \text { in } \mathrm{C} 2}}} .
\end{aligned}
$$

In Figure 5.3(a), We observe a significant overdensity in the center because of cluster galaxy population. The filament overdensity is not directly observable in this figure, due to the very small density contrast of the filaments.

Because more than $90 \%$ of the galaxies observed in the FL region come from foreground and background populations, the properties of filament galaxies would not be distinguishable without foreground/background subtraction. To get the pure properties of galaxy filaments, like color distribution and luminosity distribution, we first get such distributions of the total galaxy population in the FL region and the $\mathrm{C} 1$ region, and then subtract the $\mathrm{C} 1$ region count from the FL region count. For example, to get the color distribution of pure filament galaxies at one redshift bin, we first get color distribution of the FL region with

$$
f_{\mathrm{FL}}(g-r)=\frac{\sum_{(i, j) \text { in FL }} \sum_{k} g_{z}\left(X_{i}, Y_{j},\left(M_{r}\right)_{k},(g-r)\right)}{\sum_{(i, j) \text { in FL }} 0.49\left(h^{-1} \mathrm{Mpc}\right)^{2}}
$$


and color distribution of the $\mathrm{C} 1$ region with

$$
f_{\mathrm{C} 1}(g-r)=\frac{\sum_{(i, j) \text { in } \mathrm{C} 1} \sum_{k} g_{z}\left(X_{i}, Y_{j},\left(M_{r}\right)_{k},(g-r)\right)}{\sum_{(i, j) \text { in } \mathrm{C} 1} 0.49\left(h^{-1} \mathrm{Mpc}\right)^{2}},
$$

and then subtract $f_{\mathrm{C} 1}(g-r)$ from $f_{\mathrm{FL}}(g-r)$ as in,

$$
f_{\mathrm{pfl}}(g-r)=f_{\mathrm{FL}}(g-r)-f_{\mathrm{C} 1}(g-r)
$$

$f_{\mathrm{pfl}}(g-r)$ is the color distribution of pure filament galaxies after removing foreground/background galaxy contents. In Equation 5.13 and Equation 5.14, the $0.49\left(h^{-1} \mathrm{Mpc}\right)^{2}$ factor is in the denominators because one pixel in the $x$ and $y$ dimension of $g\left(x, y, M_{r}, g-r\right)$ is $0.7 h^{-1} \mathrm{Mpc} \times 0.7 h^{-1} \mathrm{Mpc}$. This subtraction is also illustrated in Figure 5.5. To acquire properties of cluster galaxies for comparison with filament galaxies, similar subtraction between the CL region and the $\mathrm{C} 2$ region is applied:

$$
\begin{aligned}
f_{\mathrm{CL}}(g-r) & =\frac{\sum_{k} c l_{z}\left(\left(M_{r}\right)_{k},(g-r)\right)}{0.49\left(h^{-1} \mathrm{Mpc}\right)^{2}}, \\
f_{\mathrm{C} 2}(g-r) & =\frac{\sum_{(i, j) \text { in } \mathrm{C} 2} \sum_{k} g_{z}\left(X_{i}, Y_{j},\left(M_{r}\right)_{k},(g-r)\right)}{\sum_{(i, j) \text { in } \mathrm{C} 2} 0.49\left(h^{-1} \mathrm{Mpc}\right)^{2}}, \\
f_{\mathrm{pcl}}(g-r) & =f_{\mathrm{CL}}(g-r)-f_{\mathrm{C} 2}(g-r) .
\end{aligned}
$$

Here, $f_{\mathrm{pcl}}(g-r)$ is the color distribution of pure cluster galaxies.

\subsubsection{Justification on Using C1/C2 for Foreground/Background Sub- traction}

When using $\mathrm{C} 1 / \mathrm{C} 2$ for foreground/background subtraction, a concern arises that $\mathrm{C} 1 / \mathrm{C} 2$ not only contain foreground/background galaxies, but also filaments which are not in the FL region. In this section, we show that such scenarios do exist but the detection of filament galaxies is still effective.

Indeed, more often than not, galaxy filaments also appear in regions other than the FL fields because each cluster is typically connected to more than one neighbor. In Figure 5.6(a), we show the distribution of pair connections for each cluster in our cluster pair catalog. Most clusters have more than 5 pair connections, and some of them even have 
(a)

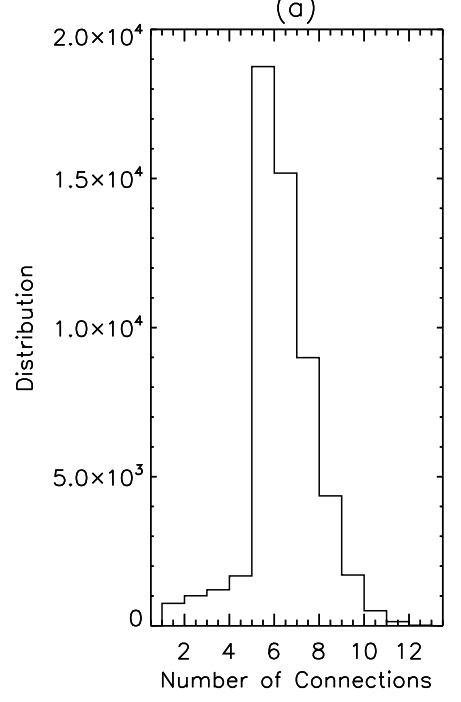

(b)

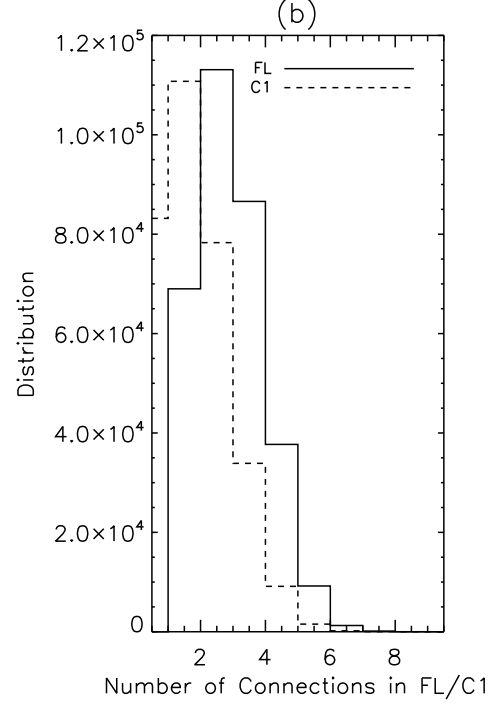

(c)

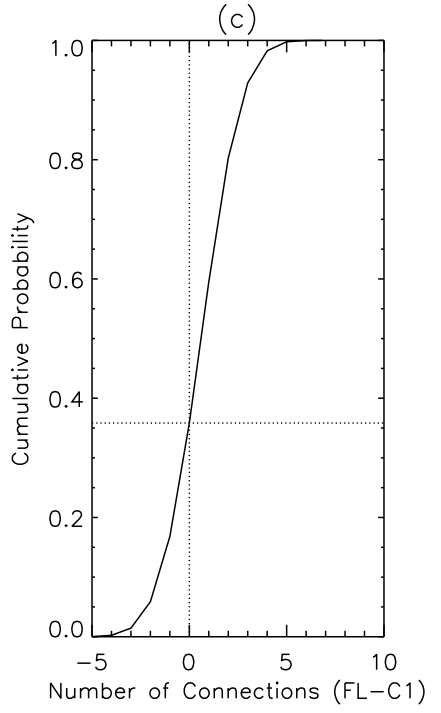

Figure 5.6: (a) Distribution of the number of pair connections each cluster have in the cluster pair catalog. Most clusters are connected to more than 5 other clusters. (b) Distribution of the number of cluster pair connections in the $\mathrm{C} 1$ region (dashed line) and the FL region (solid line). There is no cluster pair connection in $\mathrm{C} 1$ for $30 \%$ of the time, and the average number of cluster pair connections in $\mathrm{C} 1$ is lower than that in FL. Meanwhile, the FL region always contain at least one cluster pair connection since one cluster pair is always aligned along $y=0$ at the FL side. (c) Cumulative probability distribution of the difference between number of cluster pair connections in FL and C1. C1 is unlikely to have equal or more cluster pairs than FL $(\sim 35 \%)$. The horizontal and vertical dotted lines indicate where number of cluster pair connections in FL and $\mathrm{C} 1$ are equal. 
more than 10 pair connections. However, the probability of one cluster having a filament connection in $\mathrm{C} 1$ region is relatively low. In Figure 5.6(b), we show the distribution of cluster pair connections appeared in $\mathrm{C} 1$ and FL while placing one cluster pair on the $y=0$ axis in the FL region. About $30 \%$ of the clusters do not have cluster pair connection appearing in $\mathrm{C} 1$, and the number of cluster pair connections in $\mathrm{C} 1$ tend to be smaller than this number in FL. In Figure 5.6(c), we show the cumulative probability distribution of the difference between number of cluster pair connections in FL and $\mathrm{C} 1$. The probability of $\mathrm{C} 1$ having more or or equal number of cluster pair connections than the FL region is less than $40 \%$. On average, the FL region contains $1.091 \pm 0.007$ more cluster pair connections than the $\mathrm{C} 1$ region. Therefore, the FL region always tend to contain more filament galaxies than the $\mathrm{C} 1$ region, and using $\mathrm{C} 1$ for foreground/background subtraction would still leave proper galaxy counts similar to stacking pure inter-cluster galaxies.

Similarly to $\mathrm{C} 1$, the $\mathrm{C} 2$ region would also contain cluster-pair field galaxies. Using $\mathrm{C} 2$ for cluster foreground/background subtraction will then remove cluster-pair field galaxy count in addition to foreground and background galaxy count. However, because of the very high galaxy overdensity in clusters, the absence of such a population have negligible influence $(\sim 1 \%)$ and won't change the main conclusions of this chapter.

Another concern about our method of searching for filaments between cluster pairs is that since clusters tend to cluster, the filament signal we are seeing might come from clusters which cluster between cluster pairs. We compare the number of clusters two cluster finders, gmBCG (Hao et al., 2010) and redMaPPer (Rykoff et al., 2013), find around cluster pairs and between cluster pairs. We notice that although clusters might have slightly higher chance to appear between cluster pairs than appearing randomly around them, this clustering of clusters effect contributes to $<5 \%$ of the filament signal we observe.

\subsubsection{Null Test with Random Cluster Pair Re-Positioning}

To test the robustness of our algorithm, we randomly translate the angular coordinates of the cluster pairs and rerun our algorithm. We expect to get a stack of galaxies which are completely flat with no overdensity either at the "cluster center" or in the "filament region".

\subsubsection{Random Cluster Pair Re-Positioning}

For one cluster pair in the 160,954 pre-masked cluster pair catalog, we first generate a random point inside the angular region $110^{\circ}<\alpha<260^{\circ}$ and $0^{\circ}<\delta<60^{\circ}$. We then translate the angular coordinates of the two clusters in this cluster pair so that the left cluster is laid on the generated random point. After randomly re-position every cluster 
pair in the 160,954 pre-masked cluster pair catalog, we mask out these pairs which fall into regions with dust extinction in r-band larger than 0.4 , and make a new pair catalog which will be referred as the randomly re-positioned pair catalog. This new pair catalog and the original cluster pair catalog overlaps significantly in sky coverage, and have similar physical separation distributions, as well as similar cluster pair count in each redshift bin.

\subsubsection{Galaxy Set Profile of Randomly Re-positioned Pairs}

We run the algorithm described in Section 5.2.2 and Section 5.2.3 with the randomly repositioned pair catalog, and get the $S(x, y)$ contour map as shown in Figure 5.3(b), and the distributions of galaxy number count per $\left[h^{-1} \mathrm{Mpc}\right]^{2}$ in the FL region and the $\mathrm{C} 1$ region as shown in Figure 5.4. We do not observe an overdensity either in the filament region or in the cluster region when stacking galaxy sets of randomly re-positioned pairs. We therefore state that the treatment of sky coverage geometry in our algorithm is proper, and the filamentary as well as cluster overdensity observed in Section 5.2.3 are real attributes of cluster pair fields.

\subsection{Results}

\subsubsection{Color Distributions}

This section studies the $g-r$ color evolution of filaments at $0.10<z<0.42$. For SDSS filters, despite the $4000 \AA$ break transition from g-band to r-band around $z \sim 0.38$, practically, $g-r$ works almost equally well for separating blue late-type galaxies from red early-type galaxies at $0.3<z<0.42$. Hao et al. (2010) have a detailed discussion on using $g-r$ for detecting the red sequence in cluster galaxies out to $z=0.42$.

With Equations 5.13-5.16, we compute the color distributions of galaxy filaments, $f_{\mathrm{pfl}}(g-r)$, as well as the color distributions of galaxy clusters, $f_{\mathrm{pcl}}(g-r)$, at different redshifts. Results are shown in Figure 5.7. The red sequence population is observed in both clusters and filaments. Comparing to galaxy clusters, galaxy filaments have a bimodal color distribution and a larger blue galaxy population.

More interestingly, galaxy clusters and filaments both show evidence of redshift evolution, with a blue cloud galaxy population increasing steadily from $z=0.1$ to $z=0.4$. In galaxy clusters, such evolution is observed as the Butcher-Oemler effect (Butcher \& Oemler, 1978a,b, 1984), although the exact nature of it is much debated. Many embrace this effect as testimony of hierarchical clustering (Kauffmann, 1995; Baugh et al., 1996; Ellingson et al., 2001; McGee et al., 2009; Li et al., 2009), but there are also strong voices 
(a)

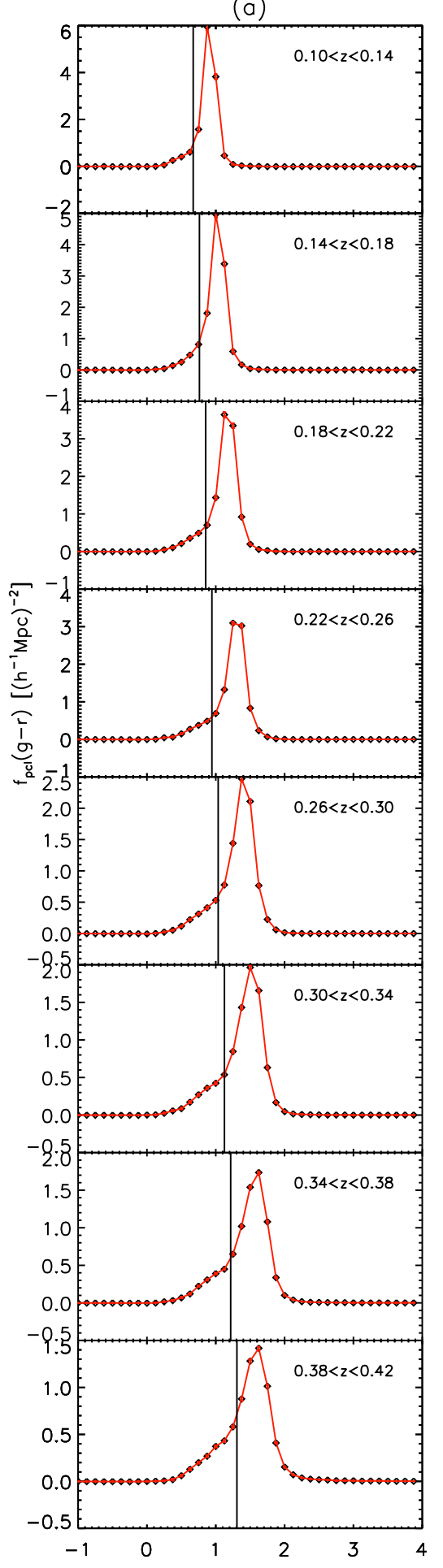

(b)

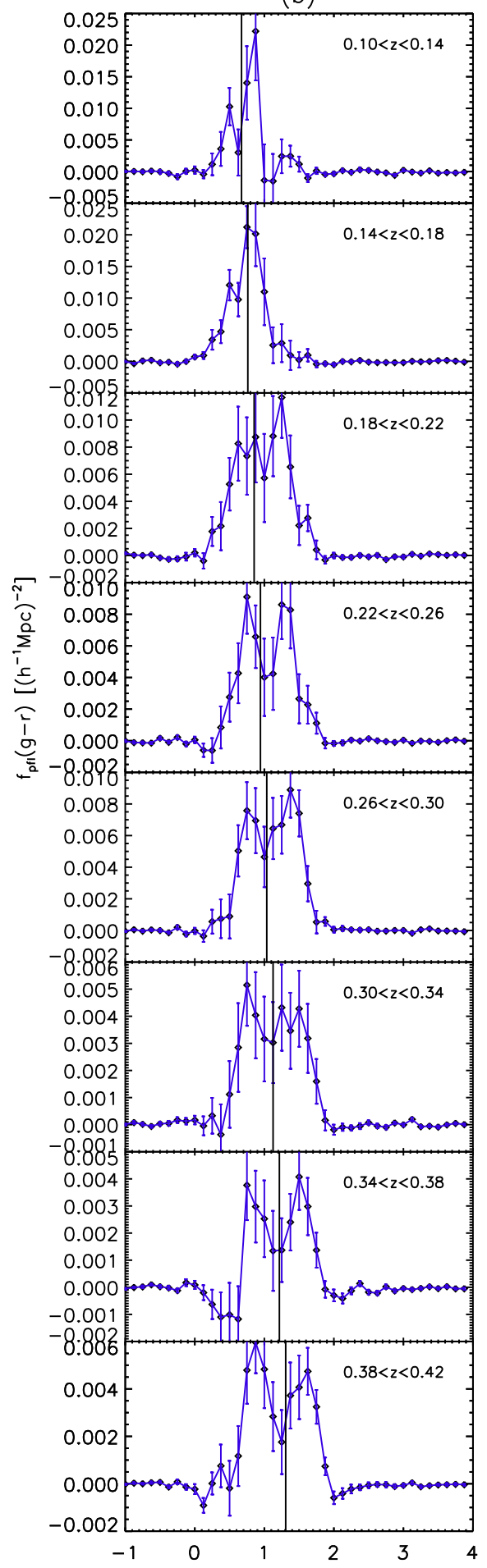

Figure 5.7: $g-r$ distributions of clusters (a) and filaments (b) in 8 redshift bins. Filaments are bimodal with their color distribution and contain a larger blue galaxy bump than clusters. Also, the filament color distribution shows evidence of redshift evolution in the form of a blue galaxy population that becomes increasingly important at higher redshift. The vertical solid lines in these plots mark the blue/red galaxy color cut used in Section 5.3.1.1. Note that the galaxy population sampled here is somewhat incomplete at $0.38<z<0.42$. 
(a)

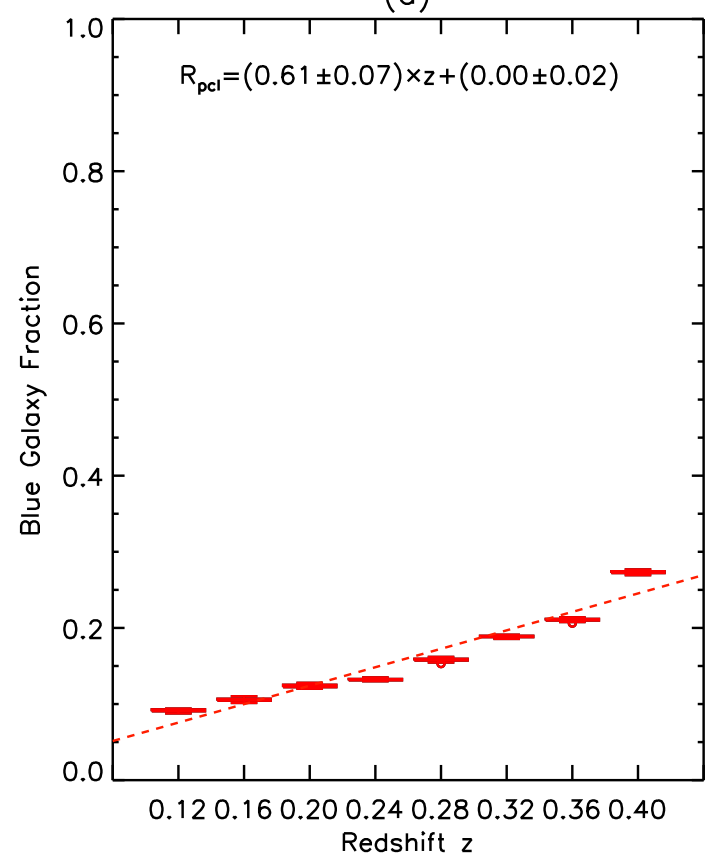

(b)

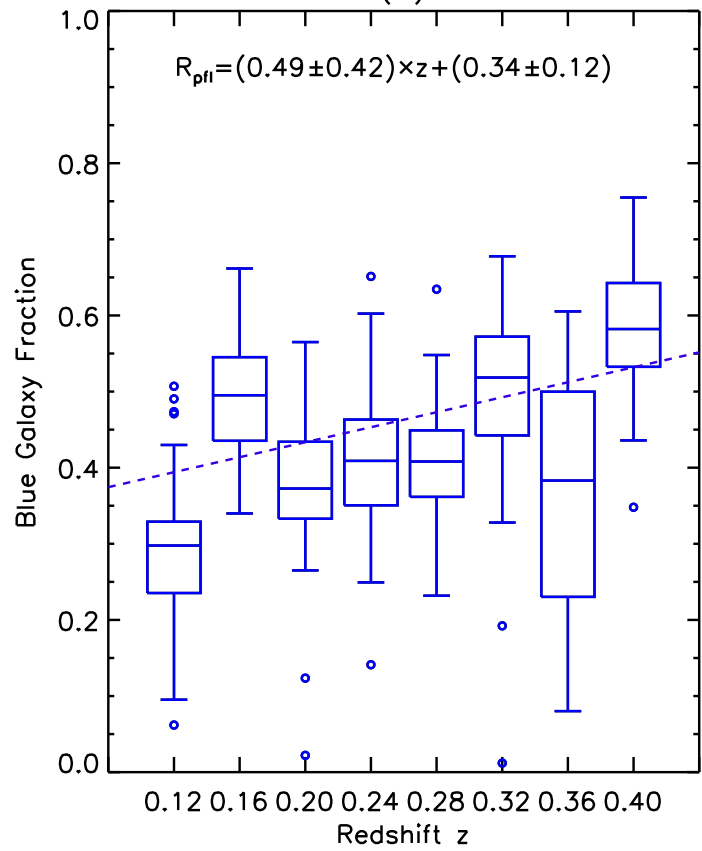

Figure 5.8: Redshift evolution of blue galaxy fraction in clusters (a) and filaments (b). These two figures show box plots of the blue galaxy fraction evaluation in clusters and filaments of 40 bootstrapped stacks at each redshift bin. The bottom and top of the boxes represent the lower and upper quartiles, and the whiskers represent the minimum and maximum values of the data, or 1.5 times the quartiles. Data points outside this range are plotted as open circles. We also fit the blue galaxy fraction linearly to the redshift (dashed lines). The blue galaxy population in clusters and filaments both seems to increase at higher redshift, but the change in filaments is less dramatic. Also note that the observed galaxy population is somewhat incomplete at $0.38<z<0.42$ (see discussion in Section 5.2.2.6).

that remain critical (Dressler, 1984; Smail et al., 1998; Andreon \& Ettori, 1999; Andreon et al., 2004, 2006), saying that either the error of measurements are underestimated, or this effect is no more than selection bias. Nevertheless, our data show a very strong redshift evolution of the cluster blue fraction and suggest that a similar effect may also exist in filaments. At redshifts $z<0.2$, the blue galaxy cloud is only a small bump comparing to the red sequence population. Moving to higher redshifts, the blue cloud becomes more prominent, taking up the more than half of the population at $z>0.28$.

\subsubsection{Blue Galaxy Fraction Evolution}

Figure 5.7 shows that filaments and clusters both contain blue and red galaxies. This figure suggests redshift evolution in the filament galaxy population. This section quantifies this 
evolution by measuring the blue galaxy fraction in filaments and clusters as a function of redshift.

To measure the blue galaxy fraction, we determine a blue/red galaxy cut to separate the blue/red galaxy population. We take the minimum point between the blue and red galaxy peak in the bimodal filament color distribution, and fit it with a linear relation to redshift. In Figure 5.7, we have these linearly fitted values marked out as a vertical solid line in both cluster and filament color distributions, and use them as the blue/red galaxy cut. We tested other blue/red galaxy cut criteria, including using local minimum in the color distributions, double gaussian mixture fitting minimums and values extrapolated from passive evolution models, but the conclusions here don't change much. We compute the blue galaxy fraction in filaments $R_{\mathrm{pfl}}$ and clusters $R_{\mathrm{pcl}}$ per redshift bin by evaluating,

$$
\begin{aligned}
R_{\mathrm{pfl}} & =\frac{\int^{s_{z}} f_{\mathrm{pfl}}(g-r) \mathrm{d}(g-r)}{\int f_{\mathrm{pfl}}(g-r) \mathrm{d}(g-r)}, \\
R_{\mathrm{pcl}} & =\frac{\int^{s_{z}} f_{\mathrm{pcl}}(g-r) \mathrm{d}(g-r)}{\int f_{\mathrm{pcl}}(g-r) \mathrm{d}(g-r)} .
\end{aligned}
$$

Here $s_{z}$ is the $g-r$ color of the separation between blue and red galaxies in this redshift bin. The integrations in the above equations are performed with a five-point Newton-Cotes integration formula on the binned color distributions.

In Figure 5.8 we show this blue galaxy fraction estimation in filaments and clusters at different redshifts. We also fit a linear evolution of the blue galaxy fraction with redshift (dashed lines) using the MPFITEXY routine (Williams et al., 2010) (dependent on the MPFIT package from Markwardt (2009)). Our measurement suggest that the blue galaxy fraction increases with redshift with a linear fitting slope of $0.61 \pm 0.07$.

For filaments, the linear fit of the blue galaxy fraction as a function of redshift slightly prefers a positive slope, $0.49 \pm 0.42$, with a significantly higher intersect, which reflects the overall bluer filament population. We change our FL and $\mathrm{C} 1$ region definition to avoid including the cluster pre-processing area $\left(>4.2 h^{-1} \mathrm{Mpc}\right.$ away from cluster center), but do not find noticeable change in this effect. This indicates the independence of this redshift evolution feature on pre-processing around clusters. We briefly discuss the implications of this result in Section 5.5.1.

\subsubsection{Luminosity Function}

In this section, we study the luminosity functions of the filament and the cluster galaxy populations. Because our data does not allow us to constrain the 3-dimensional distribution 


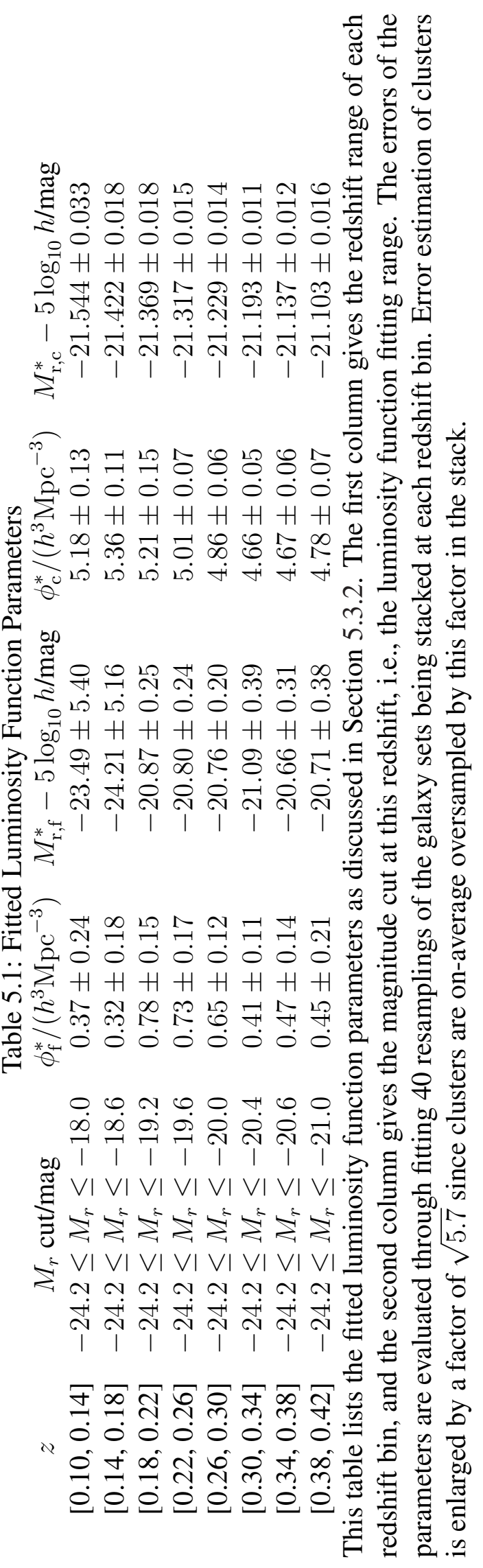



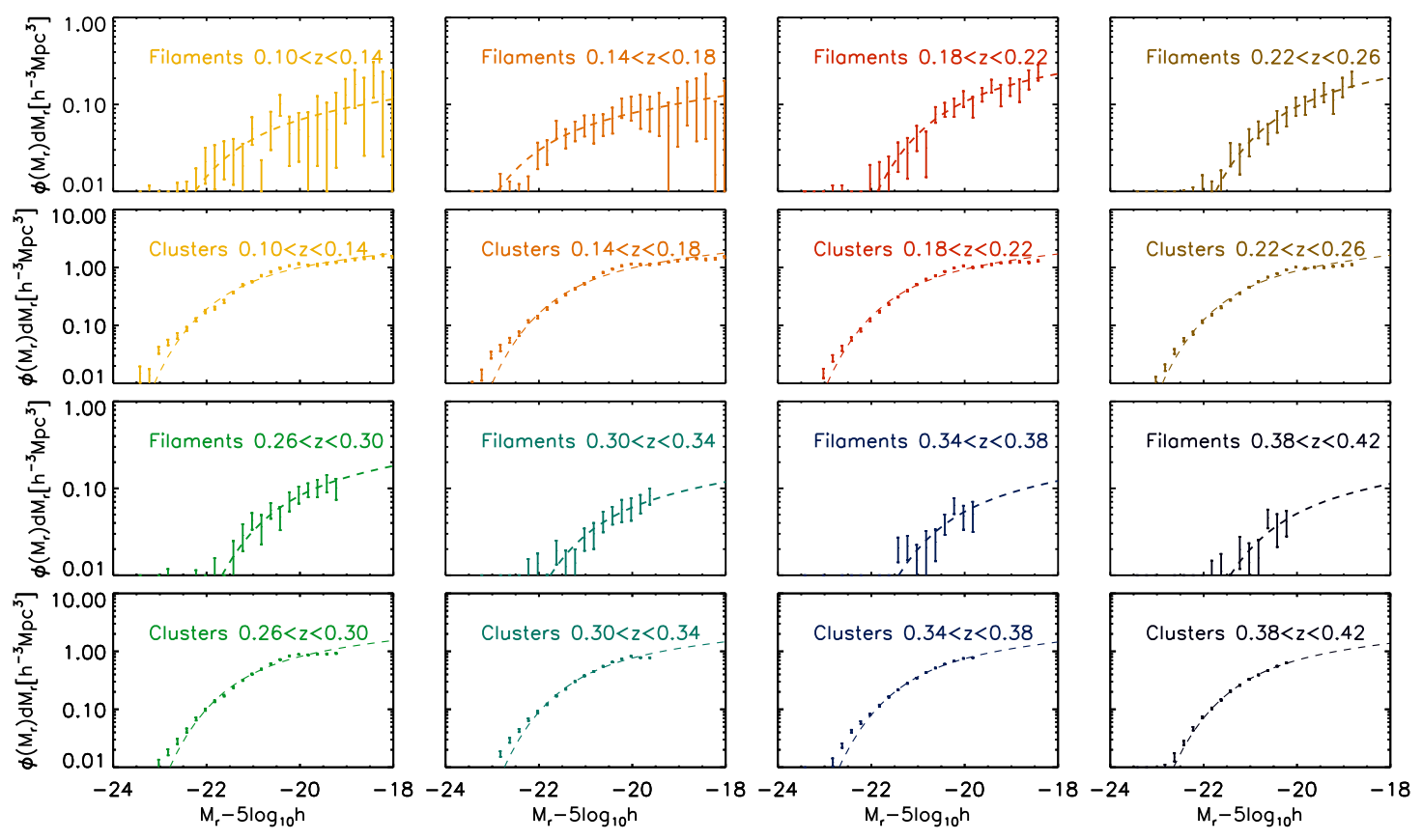

Figure 5.9: Luminosity Distributions of filaments (1st and 3rd rows) and clusters (2nd and 4th rows) and fits to the Schechter function (dashed lines) using the MPFIT package. The fitting parameters are listed in Table 5.1. We do not fit for the $M_{r}-5 \log _{10} h<-22.3$ bright end of the luminosity distributions since a LRG population component is possibly present, and it luminosity distribution tend to deviate from the Schechter function. 
of cluster and in particular of filament galaxies, we make the following assumptions when computing the number density of galaxies:

1. $N$-body simulations predict that the density profiles fall off quickly between $1 h^{-1} \mathrm{Mpc}$ and $2 h^{-1} \mathrm{Mpc}$ from the intercluster axis (Colberg et al., 2005). Thus, a single filament will typically not occupy the whole FL region. We assume that one filament occupies $1 / 3$ of the filament region defined in Section 5.2.3, and extends $\pm 1 h^{-1} \mathrm{Mpc}$ along the line of sight.

2. Since most galaxy clusters are at the scale of $1 h^{-1} \mathrm{Mpc}$, we assume that one cluster lies within the aperture of $\sqrt{x^{2}+y^{2}}<0.7 h^{-1} \mathrm{Mpc}$ and also extends $\pm 1 h^{-1} \mathrm{Mpc}$ along the line of sight. Note that this does not imply a strong triaxiality of the cluster but rather limits the choice of cluster galaxies to those found in a cylinder fully contained within the cluster.

3. We assume that the purity of the gmBCG cluster catalog is $80 \%$, which is the lower limit estimated for the gmBCG catalog (Hao et al., 2010).

4. We also assume that on average $20 \%$ of the cluster pairs in our sample are connected by filaments. This again is a lower limit, estimated from the results of Colberg et al. (2005).

The assumptions listed here are clearly only rough approximations adopted for convenience. We therefore stress that they only impact the normalization of the luminosity function and not the determination of the characteristic magnitudes as a function of environment. Consequently, we caution the reader against comparing the measured 3-d galaxy number density of filaments and clusters in this chapter to measurements obtained in other ways without taking these approximations into account.

Under the above assumptions, the luminosity distributions of filaments and clusters in one redshift bin, denoted by $\phi_{\mathrm{pfl}}\left(M_{r}\right)$ and $\phi_{\mathrm{pcl}}\left(M_{r}\right)$, respectively, are computed from 
$g_{z}\left(x, y, M_{r}, g-r\right)$ and $c l_{z}\left(M_{r}, g-r\right)$ with the following equations:

$$
\begin{aligned}
& \Phi_{\mathrm{FL}}\left(M_{r}\right)=\frac{\sum_{(i, j) \text { in FL }} \sum_{l} g_{z}\left(X_{i}, Y_{j}, M_{r},(g-r)_{l}\right)}{\sum_{(i, j) \text { in FL }} 0.49\left(h^{-1} \mathrm{Mpc}\right)^{2}}, \\
& \Phi_{\mathrm{CL}}\left(M_{r}\right)=\frac{\sum_{l} c l_{z}\left(M_{r},(g-r)_{l}\right)}{0.49\left(h^{-1} \mathrm{Mpc}\right)^{2}}, \\
& \Phi_{\mathrm{C} 1}\left(M_{r}\right)=\frac{\sum_{(i, j) \text { in } \mathrm{C} 1} \sum_{l} g_{z}\left(X_{i}, Y_{j}, M_{r},(g-r)_{l}\right)}{\sum_{(i, j) \text { in } \mathrm{C} 1} 0.49\left(h^{-1} \mathrm{Mpc}\right)^{2}}, \\
& \Phi_{\mathrm{C} 2}\left(M_{r}\right)=\frac{\sum_{(i, j) \text { in } \mathrm{C} 2} \sum_{l} g_{z}\left(X_{i}, Y_{j}, M_{r},(g-r)_{l}\right)}{\sum_{(i, j) \text { in } \mathrm{C} 2} 0.49\left(h^{-1} \mathrm{Mpc}\right)^{2}}, \\
& \phi_{\mathrm{pfl}}\left(M_{r}\right)=\frac{\Phi_{\mathrm{FL}}\left(M_{r}\right)-\Phi_{\mathrm{C} 1}\left(M_{r}\right)}{\frac{1}{3} \times \frac{1}{20 \%} \times 2 h^{-1} \mathrm{Mpc}}, \\
& \phi_{\mathrm{pcl}}\left(M_{r}\right)=\frac{\Phi_{\mathrm{CL}}\left(M_{r}\right)-\Phi_{\mathrm{C} 2}\left(M_{r}\right)}{\frac{1}{80 \%} \times 2 h^{-1} \mathrm{Mpc}} \text {. }
\end{aligned}
$$

In Figure 5.9, we plot the luminosity distributions of filaments and clusters. We also fit the luminosity distribution of filaments and clusters with a Schechter (1976) function:

$$
\phi\left(M_{r}\right) \mathrm{d} M_{r}=0.4 \ln (10) \phi^{*}\left(10^{-0.4\left(M_{r}-M_{r}^{*}\right)}\right)^{\alpha+1} e^{10^{-0.4\left(M_{r}-M_{r}^{*}\right)}} \mathrm{d} M_{r}
$$

Because our data can only put weak constraints on the faint end of the luminosity function, we chose to fix $\alpha=-1.2$, the value found by Blanton et al. (2001) and close to the measurements of Hoyle et al. (2005) and Popesso et al. (2005). The very bright end of the luminosity distribution tends to depart from a Schechter function (Yang et al., 2008). We therefore restrict our fits to galaxies with $M_{r}-5 \log _{10} h \geq-22.3$. We list the results of our fits in Table 5.1. The normalizations, $\Phi^{*}$ and r-band characteristic magnitude, $M_{r}^{*}$, of filaments and clusters are denoted by $\phi_{\mathrm{f}}^{*}, M_{\mathrm{r}, \mathrm{f}}^{*}$ and $\phi_{\mathrm{c}}^{*}, M_{\mathrm{r}, \mathrm{c}}^{*}$, respectively.

In two individual redshift bins at $0.10<z<0.14$ and $0.14<z<0.18$ our measurement of $M_{\mathrm{f}}^{*}$ has large errors. Visual inspection of these fits suggests that the fixed faint end slope may not be a good representation of the data in these bins. Especially in the low redshift bins the data, however, is too noisy to leave the faint end slope as a free parameter. We choose to ignore these two bins in our subsequent discussion and analysis. 
Table 5.2: $\Sigma_{\mathrm{pfl}}\left[\left(h^{-1} \mathrm{Mpc}\right)^{-2}\right]$ versus richness at two redshift ranges

$\begin{array}{ccc}\text { Richness range } & 0.10 \leq z<0.26 & 0.26 \leq z<0.42 \\ 8 \leq \text { Richness }<10 & 0.053 \pm 0.030 & 0.012 \pm 0.020 \\ \text { Richness } \geq 10 & 0.075 \pm 0.016 & 0.061 \pm 0.008\end{array}$

\subsubsection{Cluster Richness Dependence}

This section studies the dependence of filament overdensity on cluster richness. When stacking galaxy sets of clusters in the cluster pair catalog, in addition to two redshift bins $0.10<z<0.26$ and $0.26<z<0.42$, we further bin the cluster pairs into two richness bins: one bin with both clusters in the pair having richness estimation between 8 and 10 and one bin with both clusters in the pair having richness larger than 10. Here, by richness, we mean the GM_SCALED_NGALS or GM_NGALS_WEIGHTED richness estimation of the gmBCG cluster catalog, whichever is recommended for a specific galaxy cluster (Hao et al., 2010).

We evaluate the filament galaxy overdensity of different bins with a quantity $\Sigma_{\mathrm{pfl}}$, which is computed with equations:

$$
\begin{aligned}
\Sigma_{\mathrm{FL}} & =\frac{\sum_{(i, j) \text { in FL }} \sum_{k, l} g_{z}\left(X_{i}, Y_{j},\left(M_{r}\right)_{k},(g-r)_{l}\right)}{\sum_{(i, j) \text { in FL }} 0.49\left(h^{-1} \mathrm{Mpc}\right)^{2}}, \\
\Sigma_{\mathrm{C} 1} & =\frac{\sum_{(i, j) \text { in } \mathrm{C} 1} \sum_{k, l} g_{z}\left(X_{i}, Y_{j},\left(M_{r}\right)_{k},(g-r)_{l}\right)}{\sum_{(i, j) \text { in } \mathrm{C} 1} 0.49\left(h^{-1} \mathrm{Mpc}\right)^{2}}, \\
\Sigma_{\mathrm{pfl}} & =\Sigma_{\mathrm{FL}}-\Sigma_{\mathrm{C} 1} .
\end{aligned}
$$

This quantity is the 2-d projected galaxy number count per $\left[h^{-1} \mathrm{Mpc}\right]^{2}$ of our detected filament galaxy overdensity. However, this quantity should not be misinterpreted as the typical surface number density of filament galaxies. Not every cluster pair being stacked has a filament connection between them, and single filaments typically occupy only a small subset of the FL region. Consequently, the real projected galaxy count of filaments per unit area can be significantly larger than the values given by $\Sigma_{\text {pfl. }}$ In Table 5.2, we list the $\Sigma_{\text {pfl }}$ between cluster pairs at the above richness and redshift binning. The $\Sigma_{\text {pfl }}$ between richer cluster pairs tends to be larger than the $\Sigma_{\text {pf }}$ between poorer cluster pairs. Assuming the geometrical distribution of filaments does not change with cluster richness, this result suggests that richer cluster pairs tend to be connected by higher density filaments. 


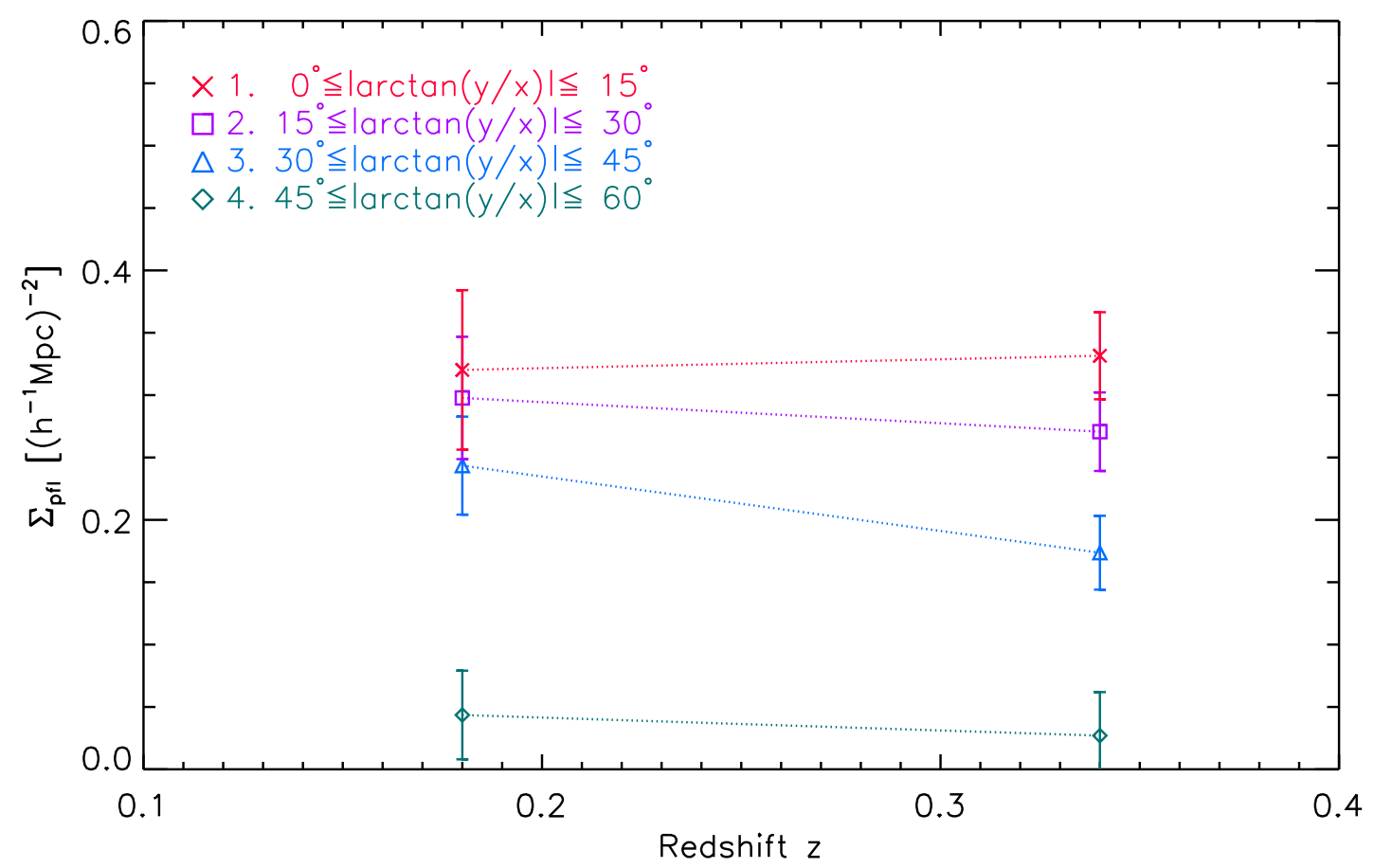

Figure 5.10: The 2-d projected galaxy number density of filaments, $\Sigma_{\mathrm{pf}}$, with FL and C1 boxes placed at different extension angles. The exact definition of FL and C1 are listed in Table 5.3. Filament galaxies still can be seen at increasing angles off the intercluster pair axis, but eventually die out when the extension angle from this axis is larger than $45^{\circ}$.

\subsubsection{Spatial Distribution}

Galaxy filaments can be warped and do not always strictly lie along the intercluster axis (Pimbblet et al., 2004; Colberg et al., 2005). Therefore, significant filamentary overdensities still can be seen off the intercluster axis. In this section, we rotate the FL region and the $\mathrm{C} 1$ region away from the intercluster axis as described in Table 5.3 and compare the surface number density of galaxies $\Sigma_{\mathrm{pfl}}$ at two redshift ranges $[0.10,0.26]$ and $[0.26,0.42]$. Our result is shown in Figure 5.10. As we move away from the intercluster axis, the observed filament population gradually falls off. At $\theta>45^{\circ}$, the filament galaxy overdensity is almost consistent with zero.

\subsection{Detection of Filaments in Simulation Data}

As further test of our method as well as for comparison with simulation data, we run our algorithm on the Millennium simulation (Springel et al., 2005), a large volume, high resolution N-body simulation under $\Lambda \mathrm{CDM}$ cosmology. This simulation includes information 


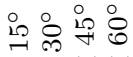

$\frac{V}{\overline{8}} \frac{V}{8} \frac{V}{8} \frac{8}{8}$

के क्रक्त

금ำ

$\underset{0}{0}$

|| || $\|$ \|

으으요

VIVIVIVI

용 is

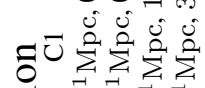

-

\& i i i

$\Xi \vee \vee \vee v$

D $\forall \forall \forall \&$

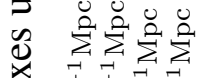

ช 111 1

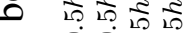

已 위윙ำ

สิ

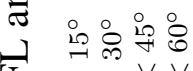

II $\vee v \vee v$

Q $\bar{\delta} \frac{8}{\overline{8}} \overline{8}$

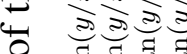

\&

일

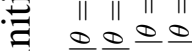

V VIVIVIV

ด : 용

نं

0 1) 1

त्ञ

$\checkmark \vee v \vee$

\&

$\checkmark \vee \vee V$

苔莞芯芯

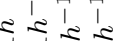

बi

产 
of dark matter structures, i.e., Friends-of-Friends (FOF) groups, and dark matter substructures. It also tracks the merging history of dark matter structures and substructures for galaxy formation simulation. For our purpose, we use the all sky lightcone realization of this simulation constructed with methods fully described in Henriques et al. (2012) and Blaizot et al. (2005), under the galaxy formation simulation of Guo et al. (2011).

Due to differences between these simulation data and SDSS observational data, we make a few adjustments of our methods before running our algorithm. First, instead of using gmBCG clusters to build the cluster pair catalog, we search for pairs of central BCGs in cluster-sized FOF groups with $M_{200}$ (the mass within the radius where the enclosed average density is 200 times the critical density of the simulation) larger than $10^{14} M_{\odot}$. We make this adjustment because running the gmBCG cluster finder on these simulations does not yield satisfying purity and completeness, possibly due to the lack of clear color bimodality in the simulated galaxy population. Secondly, the simulation galaxy catalog has a apparent magnitude limit in i-band at $i=21.0 \mathrm{mag}$. Therefore, when making the brightness cut to ensure fair comparison of the galaxy population at different redshifts (as described in Section 5.2.2.6), the luminosity limit is adjusted to $-25.3 \leq M_{i} \leq-21.9$. This luminosity limit is above the $i=21.0 \mathrm{mag}$ apparent magnitude limit at $z=0.38$. For a fair comparison, we also rerun our codes with SDSS data under the new luminosity cut. When comparing results from simulation data and SDSS data in this section, all the results presented are under this new luminosity cut.

In Figure 5.11, we show the color distributions of filament galaxies in the Millennium simulations compared to those in the SDSS data. As noted by Guo et al. (2011), the simulation seems to contain too many red sequence galaxies in filaments, and the fraction of the blue galaxies does not match that seen in SDSS.

In addition, filament galaxies in the simulation appear to be fainter than observed in SDSS. In Figure 5.12, we show the luminosity distributions of filament galaxies in simulation and SDSS, and fit them to a Schechter function with the faint end slope fixed to be $\alpha=-1.2$, as having been discussed in Section 5.3.2. The fit parameters of the distributions are listed in Table 5.4. Filament galaxies in simulation appear to have a fainter characteristic magnitude in i-band than in SDSS. Possibly because of the magnitude cut $-25.3 \leq M_{i} \leq-21.9$ being too shallow, the Schechter function fittings yield smaller estimations of $\phi^{*}$ than Section 5.3.2. 
(a)

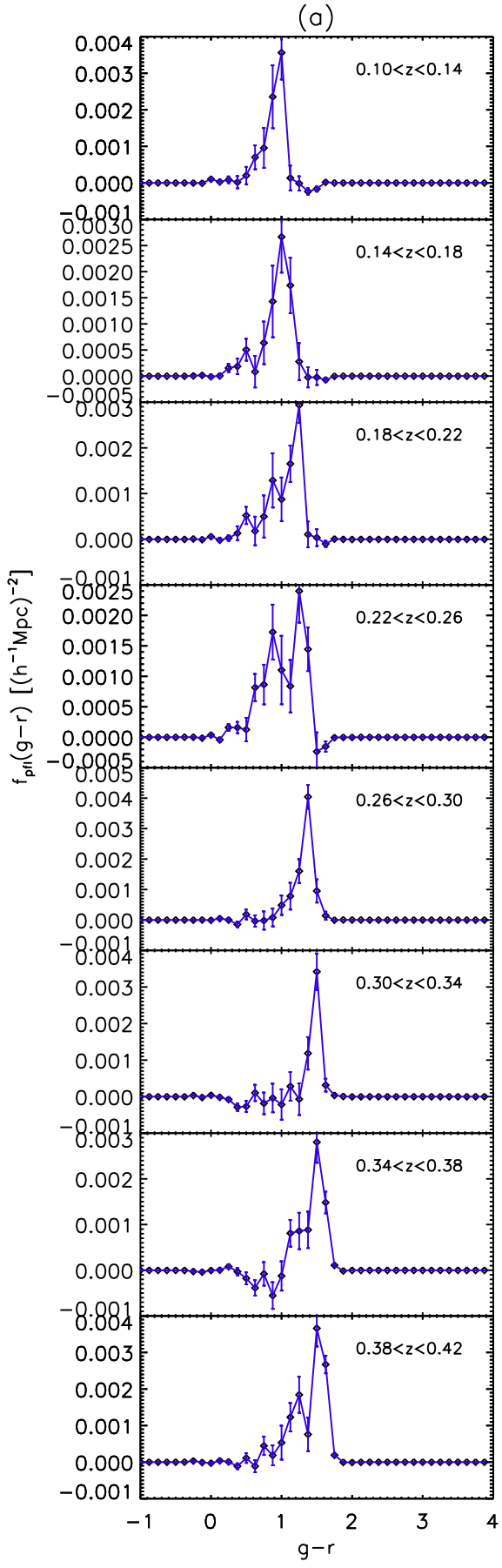

(b)

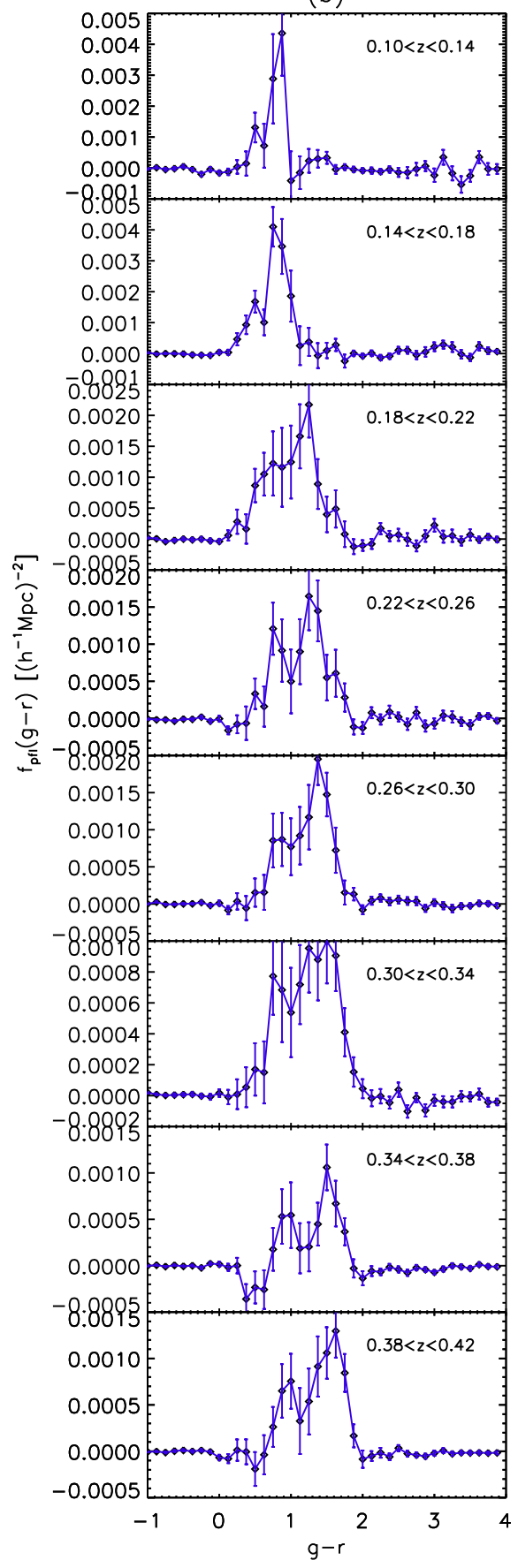

Figure 5.11: $g-r$ distributions of filaments at different redshift bins in simulation (a) and SDSS (b). Comparing to SDSS data, the simulation is over-populated with red galaxies. Note that the galaxy population is incomplete at $0.38<z<0.42$ (See discussion in Section 5.2.2.6) and also (b) differs from Figure 5.7 (b) because of the different luminosity cut applied. 

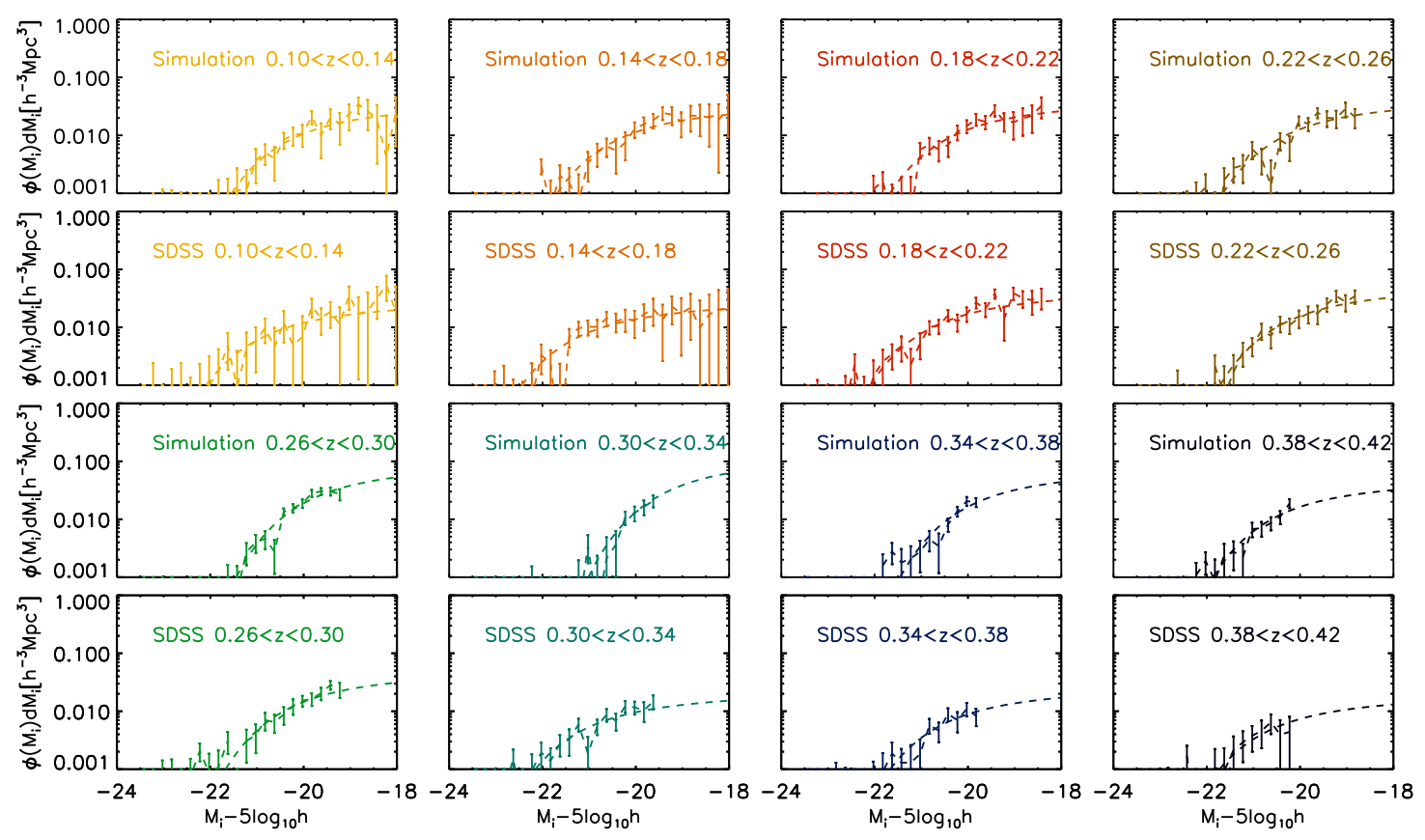

Figure 5.12: Luminosity distributions of filament galaxies in simulation (1st and 3rd rows) and SDSS (2nd and 4th rows) and their Schechter function fits (dotted lines).The Schechter function fit has a fixed faint end slope, and the rest of the fitting parameters are listed in Table 5.4. The characteristic magnitude of the fitted Schechter function is brighter in simulation than in SDSS, indicating overpopulation of bright galaxies in the simulation. 


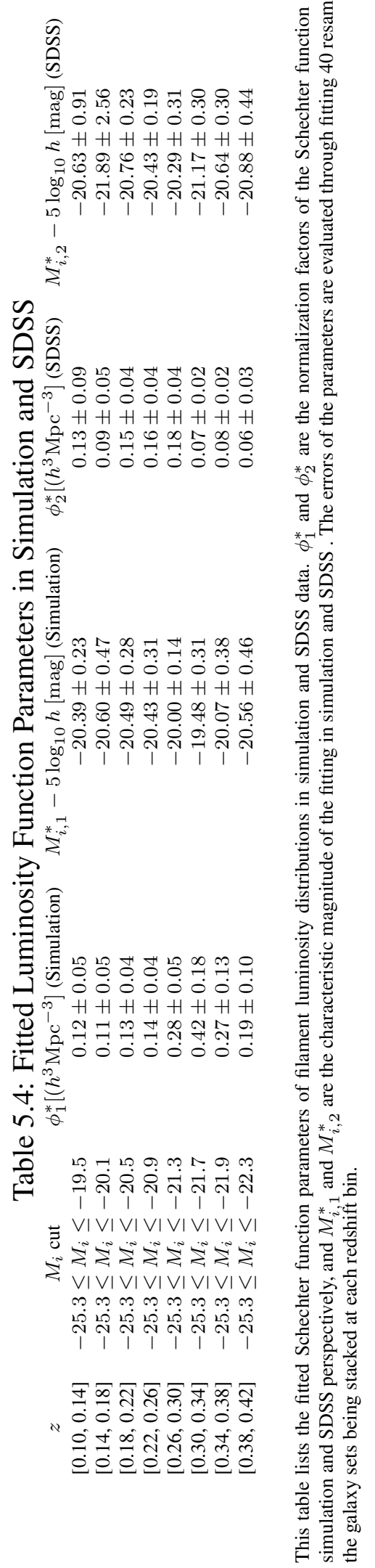




\subsection{Summary and Discussion}

In this chapter we developed an algorithm to detect and study the properties of galaxies in filaments statistically. Our method makes use of the fact that close pairs of galaxy clusters have a large probability of being connected by filaments. This is a robust prediction of numerical simulations of structure formation (e.g., Colberg et al., 2005) and has been confirmed observationally in galaxy redshift surveys (e.g., Pimbblet et al., 2004). This allows us to study filament galaxies statistically without the need to spectroscopically identify them. Our algorithm is applicable to all photometric surveys, in which large numbers of galaxy clusters can be found. As a result we can study the properties of representative filament galaxies out to much higher redshifts than is currently possible with wide-field spectroscopic surveys.

We applied our new method to the gmBCG galaxy cluster catalog and the photometric SDSS DR8 BOSS galaxy catalog. Although the projected filament galaxy densities exceed that of the surrounding field by only $\sim 2 \%$, we can detect these relative overdensities at high significance (often $\sim 5 \sigma$ ) in redshift bins of thickness $\Delta z=0.04$ at $0.10<z<0.42$. The limit of this redshift range is governed by the gmBCG cluster finding algorithm and the small survey volume in the local Universe at the low redshift end, and by the flux limit of SDSS at the high redshift end. Using data from deep wide-field surveys like the Dark Energy Survey ${ }^{1}$ will allow us to push this method to higher redshifts.

As an application of our method, we study the color and luminosity distributions of galaxy filaments, as well as the dependence of the filament richness on the richness of the clusters to which they are connected. Finally, we put limits on the warping angles of large-scale structure filaments.

As verification additional to that already presented in Section 5.2, we also apply our algorithm to the Millennium Simulation (Springel et al., 2005). These tests support the efficacy of our methods and also confirm previous suggestions that the Millennium Simulation is overpopulated with red galaxies (Guo et al., 2011).

Finally, this stacking approach to studying filaments is not only applicable with optical galaxy data, but can also be applied to weak lensing measurements, Sunyaev-Zeldovich effect observations, and X-ray emission from the warm-hot intercluster medium, provided appropriately large survey data exist. We look forward to its broader application in the era of combined data from deep wide-field optical, SZ, and X-ray surveys.

\footnotetext{
${ }^{1}$ http://www . darkenergeysurvey . org/
} 


\subsubsection{Color Evolution}

We find that filament galaxies have a clear bimodal color distribution. At redshift $0.10<$ $z<0.14$, the filament $g-r$ color distribution is qualitatively comparable to the $u-r$ color distribution of bright wall galaxies at $z<0.107$ presented in Hoyle et al. (2012): both being bimodal, and the blue cloud galaxy taking up $\sim 1 / 3$ of the whole population.

We find clear indications for a Butcher-Oemler effect in galaxy clusters at very high significance. Fitting a linear function to the blue galaxy fraction we find that it increases with redshift with a slope of $0.61 \pm 0.07$ over the redshift range studied. This is comparable to the results of Hansen et al. (2009), which showed that from $z=0.28$ to $z=0.2$, there is $\mathrm{a} \sim 5 \%$ decrease in the blue galaxy fraction, which our linear fit agrees with. Care needs to be exercised when comparing our measurement to many other works studying the Butcher-Oemler effect, though. For example, Margoniner et al. studied the Butcher Oemler effect with 295 Abell clusters, and showed that the blue fraction in these clusters increases with a slope of $1.24 \pm 0.07$ at redshift range $z<0.4$. Since Margoniner et al. adopted different cluster aperture $\left(0.49 h^{-1} \mathrm{Mpc}\right.$ instead of $\left.0.7 h^{-1} \mathrm{Mpc}\right)$, brighter magnitude limit $\left(M_{r}^{*}-1<M_{r}<M_{r}^{*}+1\right.$, which is approximately $-22.2<M_{r}<-19.2$ according to our measurements of $M_{r}^{*}$ ) and completely different blue/red galaxy cut (rest frame $g-r$ color 0.2 magnitude fainter than the red sequence ridgeline), it is not particularly surprising that we have different estimation on the blue galaxy fraction evolution strength, but a more specified study on this effect in clusters may wish to refine the choice of cluster aperture, brightness limit, and blue/red galaxy cut used here.

We marginally detect an evolution in the blue fraction of filament galaxies with a linear slope of $0.49 \pm 0.42$. This indicates that there might be a trend that the population of blue galaxies in filaments is increasing at higher redshift, but this redshift evolution feature is weaker than in clusters. The implications of such an observation are complicated, though. Should galaxy filaments be considered as ensemble of galaxy groups and galaxies in less dense environments, our observation can be the result of a Butcher-Oemler effect in galaxy groups, as discussed in Li et al. (2012). Li et al. measured the red/blue galaxy fraction in galaxy groups at different mass ranges and found that galaxy groups of all different masses show a trend of increasing blue galaxy fraction at higher redshifts. Their

measurements of the blue galaxy fraction in galaxy groups at their lowest total steller mass bin $\log \left(M_{*, \operatorname{grp}} / M_{\odot}\right)<11.2$ are $\sim 48 \%$ at $z=0.25$ and $\sim 52 \%$ at $z=0.35$, is consistent with our interpolated values $\sim 46.3 \%$ at $z=0.25$ and $\sim 51.2 \%$ at $z=0.35$. The possible redshift evolution of the blue galaxy fraction in filaments can also be a "by-product" of changes in filament galaxy contents or morphologies at different redshifts. Our results on the evolution of the blue galaxy fraction in filaments are currently limited by the signal-to- 
Table 5.5: SDSS r-band characteristic magnitude measurements

$$
\begin{array}{cc}
\text { Filament } M_{r}^{*} \text { (our measurement) } & -20.85 \pm 0.11 \\
\text { Blanton et al. (2001) } M_{r}^{*} & -20.83 \pm 0.03 \\
\text { Montero-Dorta \& Prada (2009) }{ }^{0.1} M_{r}^{*} & -20.71 \pm 0.04^{a}
\end{array}
$$

${ }^{a}$ Note that this magnitude is measured for the SDSS r-band blue shifted by 0.1 , i.e., ${ }^{0.1} r$. (Blanton et al., 2003a,b), and are not directly comparable to the rest in the table. At $z=0.1$, the K-correction to such bands are $-2.5 \log _{10} 1.1=-0.1035$, but the K-correction for our system at $z=0.1$ ranges from -0.06 to 0.37 . Here, we make a simple assumption that there is a uniform offset between the galaxy absolute magnitude measured in the ${ }^{0.1} r$ band and the regular $r$ band. Since the the K-correction we use at $z=0.1$ scatters around 0.08 , which is the K-correction for galaxies of $z=0.1$ and $g-r=0.8$ using the Chilingarian et al. (2010) approximation, we assume the offset between the two systems to be 0.18 . The r-band characteristic magnitude measured in Montero-Dorta \& Prada (2009) is now closer to our filament measurement.

noise ratio per redshift bin. Future deep surveys will give us a longer lever arm in redshift to study this effect in more detail. We also expect that improvements in galaxy cluster selection will lead to an enhanced catalog purity at all redshifts. This will improve the SNR at all redshifts.

\subsubsection{Luminosity Function}

We measured the characteristic r-band magnitude of cluster and filament galaxies in redshift bins by fitting a standard Schechter luminosity function with a fixed faint end slope of $\alpha=$ -1.2. For cluster galaxies, Popesso et al. (2005), who studied the RASS-SDSS clusters to $z=0.26$, is a suitable comparison to our work. Their measurements of the cluster $\mathrm{r}-$ band characteristic magnitude at $z<0.26$ are $-21.35 \pm 0.19$ mag or $-21.40 \pm 0.20$ mag, depending on the choice of background. This is in good agreement with our measurements. An increase of $M_{\mathrm{r}}^{*}$ of cluster galaxies with redshift is seen at high statistical significance. This result, however, may be affected by a potential redshift dependence of the purity of the gmBCG catalog, as well as a redshift dependent richness threshold in the cluster finder. A detailed study of these factors is beyond the scope of the present work and the redshift evolution of $M^{*}$ should be treated with caution.

In redshift bins where we obtain reliable measurements, the r-band $M^{*}$ of filament galaxies is significantly lower than that of cluster galaxies. We find no significant redshift evolution of $M_{\mathrm{r}, \mathrm{f}}^{*}$.

When we stack the luminosity distributions of filament galaxies over $0.18<z<0.42$, 
we obtain a characteristic luminosity of filament galaxies of $M_{\mathrm{r}, \mathrm{f}}^{*}=-20.85 \pm 0.11 \mathrm{mag}$. This value is compatible with two r-band $M^{*}$ measurements (listed in Table 5.5) of SDSS galaxies, in which galaxies were not separated by their environment. This re-enforces the result of Aragón-Calvo et al. (2010) that most matter and thus most galaxies live in filaments and not in clusters. Consequently, the filament environment is expected to dominate globally averaged measurements. Our value is also close to the r-band $M^{*}$ measurement of wall galaxies in Hoyle et al. (2005), who found $M^{*}=-20.62 \pm 0.08$ mag. The filament galaxies in this work fall into the category of wall galaxies in Hoyle et al. (2005) (galaxies in denser environments than extremely underdense cosmic voids). Not surprisingly, filament galaxies are significantly brighter than void galaxies, whose r-band $M^{*}$ was measured to be $-19.74 \pm 0.11$ mag by Hoyle et al. (2005).

\subsubsection{Filament Richness and Geometry}

We find that richer clusters are connected to richer filaments. This is consistent with description of structure formation given by Bond et al. (1996). In this picture, superclusters with pronounced filaments between their massive constituent clusters are the result of rare initial density peaks. In turn, less massive clusters were "disadvantaged" from the beginning by being more isolated and having fewer and less dense filaments connected to them. Because we select up to 5 neighbors for any cluster to form cluster pairs with, independent of the cluster richness, both factors - the density and the number of filaments - affects the measured surface density of galaxies. Consistent with this expectation, we find that the correlation of filament richness with cluster richness exists at two redshift ranges studied here.

Finally, we also studied the distribution of matter in what we call the filament region between the clusters. Slightly more than half of all filaments are curved and some connect the clusters at which they terminate with offsets from the intercluster axis. These reasons make it impossible to disentangle the density profile of filaments from the distribution of filament geometries in our stacks. Nevertheless, the galaxy density in the filament region should decrease as one moves away from the intercluster axis. We verified this by varying the position angle of the filament region when rotating it around the cluster center. We find that filaments are unlikely to be warped by more than $45^{\circ}$ from the intercluster axis. 


\section{CHAPTER 6}

\section{Closing Remarks}

This dissertation reports on new findings about cluster galaxy evolution from redshift 1.0 to 0. Most of them have been published as first-author papers (See discussion in Section 1.4). Compared to previous literature reports, the studies either extend to higher redshift, or improve the understanding of a previously little-understood topic. The statistical power of the data from the Dark Energy Survey (DES) and the Sloan Digital Sky Survey (SDSS) made this work possible.

Yet we are still in the rising phase of understanding cluster galaxies. SDSS has transformed our understanding about the $z<0.5$ universe in the past fifteen years. DES and a couple of other wide field surveys are actively producing deeper data sets for galaxy cluster research. The opportunity to thoroughly study the $0.5<z<1.0$ regime is now at our finger tips. Combining the data from DES, SDSS, WISE and Spitzer telescope surveys, we shall be able to make a dent in understanding the $z>1.0$ universe. The past a few years have also witnessed discoveries of new cluster galaxy populations like ultra compact dwarf galaxies (Hilker et al., 1999; Drinkwater et al., 2000; Phillipps et al., 2001; Drinkwater et al., 2003) and ultra diffuse galaxies (van Dokkum et al., 2015a,b; Koda et al., 2015; Muñoz et al., 2015; Mihos et al., 2015). Astrophysics implications of these galaxies are still being investigated. Looking further away, more ambitious optical and infrared sky surveys are being developed for the next decade. The studies presented in this dissertation are only early efforts in this wave of development.

In this chapter, I first overview the studies performed in this dissertation and then discuss follow-up opportunities for the next a couple of years.

\subsection{Recapitulation}

The dissertation starts with a new technique developed for DES on improving cluster galaxy detection and then continues to analyze cluster galaxy evolution with DES early data. The 
last part of the dissertation is devoted to characterizing the properties of cosmic filaments. DES has not covered enough area at the time the filament study was performed, so the analysis was performed with SDSS data. A recapitulation of these studies follows:

\section{- Data processing.}

Deep optical images are often crowded with overlapping objects. This is especially true in the cores of galaxy clusters, where images of dozens of galaxies may lie atop one another. Accurate measurements of cluster properties require deblending algorithms designed to automatically extract a list of individual objects and decide what fraction of the light in each pixel comes from each object. This dissertation introduces a new software tool called the Gradient And INterpolation (GAIN) based deblender. GAIN is a secondary software package that improves the separation of overlapping objects in galaxy cluster cores in Dark Energy Survey images. It uses image intensity gradients and an interpolation technique originally developed to correct flawed digital images. The dissertation describes the algorithm of the GAIN deblender and its applications, as well as modest tests of the software based on real Dark Energy Survey coadd images. GAIN helps to extract unbiased photometry measurement for blended sources and improve detection completeness while introducing few spurious detections. When applied to processed Dark Energy Survey data, GAIN serves as a useful quick fix when a high level of deblending is desired.

\section{- Central galaxy formation.}

The $\Lambda \mathrm{CDM}$ cosmology predicts a hierarchical structure formation scenario where smaller galaxies merge to form more massive ones, but galaxy stripping may suppress this procedure while in situ star formation may contribute to it. Using DES data and an X-Ray selected cluster sample from the XMM Cluster Survey, we investigate this scenario with cluster Bright Central Galaxies (BCGs). Compared with the expectation in a semi-analytical model applied to the Millennium Simulation, the observed BCGs do not grow as dramatically as expected. We incorporate the uncertainties associated with cluster mass, redshift, and BCG stellar mass measurements into analysis of a redshift-dependent BCG-cluster mass relation,

$m_{*} \propto\left(\frac{M_{200}}{1.5 \times 10^{14} M_{\odot}}\right)^{0.24 \pm 0.08}(1+z)^{-0.19 \pm 0.34}$, and compare the observed relation to the model prediction. We estimate the average growth rate since $z=1.0$ for BCGs hosted by clusters of $M_{200, z}=10^{13.8} M_{\odot}$, at $z=1.0: m_{*, B C G}$ appears to have grown by $0.13 \pm 0.11 \mathrm{dex}$, in tension at $\sim 2.5 \sigma$ significance level with the 0.40 dex growth rate expected from the semi-analytic model. We show that the buildup of extended intra-cluster light after $z=1.0$ may alleviate this tension in BCG growth rates. This 
finding illustrates the importance of astrophysics processes in galaxy cluster formation and evolution.

\section{- Red sequence galaxy formation}

Using the same sample mentioned above, we investigate the luminosity function evolution of red sequence galaxies and find that many are already in place at $0.5<z<1$.0. We develop Bayesian modeling techniques that can simultaneously constrain the dependence of luminosity function parameters on redshift and cluster mass. We notice hints of mild redshift evolution and mass dependence of the faint end slope. The characteristic magnitude also appears to vary for clusters of different masses, being brighter for more massive ones. This study probes the connection between dark matter halos and galaxies at the massive end. For cosmological studies, the results raises a concern for using cluster richness as cluster mass proxies. Since the results indicate that the number of faint red sequence galaxies possibly change with redshift and cluster mass, including these faint galaxies may result in cluster mass proxies that is dependent on cluster mass or redshifts. The redshift and cluster mass trends presented in this dissertation need to be further investigated.

\section{- Galaxy filaments.}

Galaxy filaments, which are thin structures of galaxies organized like strands or walls between galaxy clusters, provide environments for galaxy cluster formation and evolution. A large fraction of the galaxies in the universe are distributed in galaxy filaments, instead of being in the rare galaxy clusters. Characterizing the properties of filament galaxies helps understanding how cluster galaxies differ from the typical "field" galaxies.

Galaxy filaments are very difficult to study individually (e.g., see Dietrich et al., 2012) because of their low density. As they almost only exist between clusters (Colberg et al., 2005), I explore statistical analysis of filament properties through stacking cluster pairs with SDSS data. The method can detect the inter-cluster filament galaxy over-density at a significance level of $\sim 5 \sigma$ out to $z=0.40$, and allows us to study the $g-r$ color and the luminosity distribution of filament galaxies as a function of redshift. Consistent with expectation, filament galaxies are bimodal in their color distribution and contain a larger fraction of blue galaxies than clusters. Filament galaxies are also generally fainter than cluster galaxies. More interestingly, the observed filament population seems to show redshift evolution at $0.12<z<0.40$ : the blue galaxy fraction has a trend to increase at higher redshift: a filament "Butcher 
Oemler Effect". We test the dependence of the observed filament density on the richness of the cluster pairs: richer clusters are connected by higher density filaments. We also test the spatial dependence of filament galaxy overdensity: this quantity decreases when moving away from the inter-cluster axis between a cluster pair. The method provides an economical way to probe the photometric properties of filament galaxies.

\subsection{Outlook}

The analyses discussed in this dissertation can be extended in a few ways in the next a couple of years. The new cluster galaxy detection technique discussed in Chapter 2 has produced a few science opportunities upon application to DES data. The precision of the analyses presented in Chapter 3 to 5 can be improved with newer data sets from DES. It is also possible to study the investigated topics at higher redshift combining data from DES and a few other survey programs.

\section{- New science opportunities with the GAIN deblender.}

Chapter 2 of this dissertation presents a new deblending package - GAIN - that improves the detection efficiency of blended objects, and prepares them for unbiased photometry measurements. We have applied GAIN to provide a de-blending valueadded catalog for DES science verification data. The catalog has now contributed to measuring cluster photometric redshifts, measuring BCG photometry, estimating cluster richness as well as finding strong lensing clusters.

Specifically, I would like to discuss one new science opportunity on characterizing the properties of compact galaxies with this software. Compact galaxies like Ultra Compact Dwarf Galaxies (UCDs) or Compact Elliptical galaxies (CEs) are mysterious objects. Being extremely small ( $<200$ pc for UCDs, and $<600$ pc for CEs) and highly concentrated with stars, they may (Seth et al., 2014) or may not (Norris et al., 2015) contain a central black hole and do not appear to be associated with a massive dark matter halo (Tollerud et al., 2011; Mieske et al., 2013). It is likely that many of these objects are stripped cores of massive galaxies (Bekki et al., 2001, 2003; Goerdt et al., 2008; Paudel et al., 2010; Brodie et al., 2011; Pfeffer \& Baumgardt, 2013; Zhang et al., 2015a; Janz et al., 2015; Liu et al., 2015). Therefore, their distribution inside clusters may contain information about the galaxy stripping processes, which in turn helps with understanding intra-cluster light creation and cluster central galaxy 
growth. However, a couple of other mechanisms may also lead to their formation (a brief review can be found in Liu et al., 2015).

One biggest challenge toward studying these objects is that they are extremely difficult to find. Only a few hundreds (brief reviews can be found in Norris et al., 2014; Chilingarian \& Zolotukhin, 2015) have been identified. Since these objects appear around luminous galaxies, the GAIN software can be used to improve their detection efficiency.

Examining the GAIN DES science verification catalog, there exist many unresolved objects at cluster core. Preliminary size analysis shows that these objects fall into the category of UCD and CEs. A study of 17 clusters with the CLASH high precision imaging data has confirmed the phenomenon: about 10 massive UCDs are clustered around cluster core (Zhang et al. in prep.). Further carrying out the CLASH analysis with the GAIN DES catalog will enlarge the cluster sample size by $\sim 100$ times, making it possible to statistically characterize the distribution of UCDs and CEs and probe their formation mechanisms.

\section{- Optical cluster sample for cluster galaxy study.}

Chapter 3 and Chapter 4 describe studies about cluster bright central galaxies (BCGs) and cluster red sequence galaxies with an X-ray selected sample. The sample provides accurate $\mathrm{X}$-ray mass proxies and alleviates selection effects on cluster galaxies, but the results are statistically limited because of the small sample size. Further more, the redshift-dependent and mass-dependent results presented in Chapter 4 need to be further investigated to improve cluster richness estimators for DES cluster abundance cosmology studies. Future release of $\mathrm{X}$-ray cluster catalog from the $X M M$ Cluster survey $(\mathrm{XCS}$ ) is predicted to be $\sim 10$ times larger, improving the significance of the results by $\sim 3$ times (simulation predictions). Further more, it is possible to extend the work to samples of optically selected clusters.

The redMaPPer (Rykoff et al., 2014) cluster finder is one of the algorithms that are finding optically selected clusters. The algorithm has identified $\sim 1,000 z<$ 0.9 clusters in about $150 \mathrm{deg}^{2}$ DES science verification data, and $\sim 7,500 z<0.7$ clusters in about 2,500 deg${ }^{2}$ Year 1 data. DES Year 2 data that covers another 2,500 $\operatorname{deg}^{2}$ of the sky will enlarge the redMaPPer cluster sample by about 2 times. The following 3 years of DES observation will gradually build up the imaging depth, eventually allowing detecting clusters to $z=1.0$. Estimating from the comoving volume at $z=0.7$ and $z=1.0$, the final DES redMapper catalog will contain about 
30,000 to 40,000 optically-selected clusters. A sample of this size means that the constraints on BCG and cluster red sequence formation will improve by $\sim 20$ times.

A cluster sample of the above size will also allow studies of cluster galaxies that have not yet transformed onto the red sequence. These galaxies constitute a small fraction of the cluster galaxy population and their luminosity measurements are noisier. We were not able to acquire statistically meaningful results about these galaxies in (Chapter 4) with the X-ray selected sample, but it shall become possible with the redMaPPer catalogs. In addition, the optically selected clusters up to $z \sim 1.0$ can be used to study galaxy filaments to the same redshift range.

The analyses can also be conducted with clusters selected by other cluster finding algorithms (C4, VT and WAZP: Miller et al., 2005; Soares-Santos et al., 2011; Benoist, 2014).

\section{- Intra-cluster light and central galaxy star formation}

There are three processes that affect the growth of Bright Central galaxy (BCG) stellar content: BCG merging events that bring in new materials, in situ star formation that converts gas into stars, and the creation of intra-cluster light (ICL) that slows down material deposition onto the BCGs. Chapter 3 confirms that the BCG growth rate after $z=1.2$ is in tension at $\sim 2.5 \sigma$ significance level with the expectation from a merging dominated scenario, and show that the stellar content stripping from the BCGs towards forming intra-cluster light may alleviate this tension. This picture may be further tested with observational quantification of both ICL creation and BCG in situ star formation.

In fact, the work presented in Chapter 3 is not the only one that emphasizes the importance of ICL creation in BCG growth. Most structure formation models include some form of stripping between galaxies that would produce ICL, yet ICL is barely constrained by observations. Although measuring ICL individually for a large cluster sample is extremely difficult, it is possible to use a stacking technique that averages the images of thousands of clusters binned by mass or redshift to acquire statistical measurements (Zibetti et al., 2005). Based on the redMaPPer (Rykoff et al., 2014) cluster density in DES science verification data and account for cosmic dimming, the stacking technique shall allow for detecting ICL to redshift 0.7 - 0.8. Previous case to case studies, especially studies based on Hubble space telescope data (Montes \& Trujillo, 2014), will help verifying the results acquired by the stacking technique.

The BCG star formation rate can be directly measured with spectroscopic data 
(Liu et al., 2012; Groenewald \& Loubser, 2014). To date, such studies are limited to using low redshift data from SDSS or data from small observing programs. However, the spectroscopic follow-up program established between DES and OzDES is taking spectra for a few hundreds of clusters up to redshift $\sim 1.0$. The program may provide one of the largest data sets that extends to high redshift for BCG star formation study.

\section{- Galaxy clusters above redshift 1.0.}

The properties of galaxy clusters above $z=1.0$ are still little known, but on-going deep sky surveys are providing the opportunity to conduct statistical analysis. Above redshift 1.0 and below redshift 3.0, the cluster samples discovered in various programs contain a few hundreds of clusters at most (e.g., searches performed with data from the Spizter telescope: Rettura et al., 2014; Webb et al., 2015). A large sample with well understood properties will still be helpful for improving the understanding of high redshift clusters.

Combining the deep data sets from DES and existing infra-red surveys, it is possible to identify at least hundreds of clusters to redshift 1.5 and beyond. Using a stacking technique on archival data from X-ray telescopes (XMM and Chandra) and cosmic microwave background telescopes (Planck and the South Pole Telescopes) will help quantifying these clusters' gas contents.

Spectroscopic follow-ups are necessary for performing cluster redshift calibration, cluster mass calibration and also cluster galaxy evolution measurements. At redshift above 1.0, archival spectroscopic data for clusters are scarce. This may become one limiting factor for high redshift cluster study. Designated follow-up programs need to be performed on large telescopes because of the faint brightness of high redshift galaxies. Clusters discovered in a small patch of the DES footprint may become eligible for OzDES targeting.

These are some of the near-term opportunities for the topics in this dissertation. The momentum of observationally studying cluster stellar content should continue to accumulate to 2020, thanks to the development of the Large Synoptic Survey Telescope ${ }^{1}$ and the Euclid mission ${ }^{2}$. The Large Synoptic Survey Telescope aims to image 25,000 $\mathrm{deg}^{2}$ of the sky with a $8.2 \mathrm{~m}$ ground telescope starting from 2022. The Euclid is a space mission that will be launched in 2020 to image $15,000 \mathrm{deg}^{2}$ the sky. The two programs will relay the expansion of observational data availability, fueling future galaxy cluster research.

\footnotetext{
${ }^{1}$ http://www.lsst.org

${ }^{2} \mathrm{http} / / / \mathrm{www}$. euclid-ec.org
} 
The last decade has witnessed many fundamental improvements in cluster astrophysics studies, enabled by wide field surveys like SDSS. Nowadays, wide field sky observations are available in a variety of wavelength ranges. More ambitious programs like the Dark Energy Survey are smoothly running. The Large Synoptic Survey Telescope and the Euclid mission are being actively developed. It is likely that another wave of improvements still awaits us. 


\section{BIBLIOGRAPHY}

Abazajian, K. N., Adelman-McCarthy, J. K., Agüeros, M. A., et al. 2009, ApJS, 182, 543

Abell, G. O. 1958, ApJS, 3, 211

Ackermann, M., Ajello, M., Albert, A., et al. 2014, ApJ, 787, 18

-. 2015, ApJ, 812, 159

Adelman-McCarthy, J. K., Agüeros, M. A., Allam, S. S., et al. 2006, ApJS, 162, 38

Ahn, C. P., Alexandroff, R., Allende Prieto, C., et al. 2012, ApJS, 203, 21

Aihara, H., Allende Prieto, C., An, D., et al. 2011, ApJS, 193, 29

Allen, S. W., Evrard, A. E., \& Mantz, A. B. 2011, ARA\&A, 49, 409

Andreon, S. 2002, $A \& A, 382,495$

-. 2008, MNRAS, 386, 1045

Andreon, S., \& Ettori, S. 1999, ApJ, 516, 647

Andreon, S., Gargiulo, G., Longo, G., Tagliaferri, R., \& Capuano, N. 2000, MNRAS, 319, 700

Andreon, S., Lobo, C., \& Iovino, A. 2004, MNRAS, 349, 889

Andreon, S., Quintana, H., Tajer, M., Galaz, G., \& Surdej, J. 2006, MNRAS, 365, 915

Aragón-Calvo, M. A., van de Weygaert, R., \& Jones, B. J. T. 2010, MNRAS, 408, 2163

Arnaud, M., Pointecouteau, E., \& Pratt, G. W. 2005, A\&A, 441, 893

Bañados, E., Hung, L.-W., De Propris, R., \& West, M. J. 2010, ApJ, 721, L14

Bahé, Y. M., \& McCarthy, I. G. 2015, MNRAS, 447, 969

Bai, L., Yee, H. K. C., Yan, R., et al. 2014, ApJ, 789, 134

Barden, M., Häußler, B., Peng, C. Y., McIntosh, D. H., \& Guo, Y. 2012, MNRAS, 422, 449

Barkhouse, W. A., Yee, H. K. C., \& López-Cruz, O. 2007, ApJ, 671, 1471 
Baugh, C. M., Cole, S., \& Frenk, C. S. 1996, MNRAS, 283, 1361

Beard, S. M., MacGillivray, H. T., \& Thanisch, P. F. 1990, MNRAS, 247, 311

Behroozi, P. S., Wechsler, R. H., \& Conroy, C. 2013, ApJ, 770, 57

Bekki, K., Couch, W. J., \& Drinkwater, M. J. 2001, ApJ, 552, L105

Bekki, K., Couch, W. J., Drinkwater, M. J., \& Shioya, Y. 2003, MNRAS, 344, 399

Benoist, C. 2014, in Building the Euclid Cluster Survey - Scientific Program, proceedings of a conference held July 6-11 2014 at the Sexten Center for Astrophysics. Online at http://www.sextencfa.eu/images/stories/conferenze2014/buildingclusters/Building_program_talks.pdf, id. 8,8

Bergé, J., Gamper, L., Réfrégier, A., \& Amara, A. 2013, Astronomy and Computing, 1, 23

Bernardi, M., Hyde, J. B., Sheth, R. K., Miller, C. J., \& Nichol, R. C. 2007, AJ, 133, 1741

Bernardi, M., Meert, A., Sheth, R. K., et al. 2013, MNRAS, 436, 697

Bernardi, M., Meert, A., Vikram, V., et al. 2014, MNRAS, 443, 874

Bernstein, R. A., Freedman, W. L., \& Madore, B. F. 2002, ApJ, 571, 107

Bertalmio, M., Bertozzi, A., \& Sapiro, G. 2001, in Computer Vision and Pattern Recognition, 2001. CVPR 2001. Proceedings of the 2001 IEEE Computer Society Conference on, Vol. 1, I-355-I-362 vol.1

Bertalmio, M., Sapiro, G., Caselles, V., \& Ballester, C. 2000, in Proceedings of the 27th Annual Conference on Computer Graphics and Interactive Techniques, SIGGRAPH '00 (New York, NY, USA: ACM Press/Addison-Wesley Publishing Co.), 417-424

Bertin, E. 2011, in Astronomical Society of the Pacific Conference Series, Vol. 442, Astronomical Data Analysis Software and Systems XX, ed. I. N. Evans, A. Accomazzi, D. J. Mink, \& A. H. Rots, 435

Bertin, E., \& Arnouts, S. 1996, A\&AS, 117, 393

Bertschinger, E., \& Gelb, J. M. 1991, Computers in Physics, 5, 164

Biviano, A. 2000, in Constructing the Universe with Clusters of Galaxies, 1

Blaizot, J., Wadadekar, Y., Guiderdoni, B., et al. 2005, MNRAS, 360, 159

Blanton, M. R., \& Roweis, S. 2007, AJ, 133, 734

Blanton, M. R., Dalcanton, J., Eisenstein, D., et al. 2001, AJ, 121, 2358

Blanton, M. R., Brinkmann, J., Csabai, I., et al. 2003a, AJ, 125, 2348 
Blanton, M. R., Hogg, D. W., Bahcall, N. A., et al. 2003b, ApJ, 592, 819

Bleem, L. E., Stalder, B., de Haan, T., et al. 2015, ApJS, 216, 27

Bond, J. R., Kofman, L., \& Pogosyan, D. 1996, Nature, 380, 603

Bonnett, C., Troxel, M. A., Hartley, W., et al. 2015, ArXiv e-prints

Boselli, A., \& Gavazzi, G. 2014, A\&A Rev., 22, 74

Bower, R. G., Lucey, J. R., \& Ellis, R. S. 1992a, MNRAS, 254, 601

-. 1992b, MNRAS, 254, 589

Brodie, J. P., Romanowsky, A. J., Strader, J., \& Forbes, D. A. 2011, AJ, 142, 199

Brough, S., Collins, C. A., Burke, D. J., Mann, R. G., \& Lynam, P. D. 2002, MNRAS, 329, L53

Brough, S., Couch, W. J., Collins, C. A., et al. 2008, MNRAS, 385, L103

Bruzual, G., \& Charlot, S. 2003, MNRAS, 344, 1000

Budzynski, J. M., Koposov, S. E., McCarthy, I. G., \& Belokurov, V. 2014, MNRAS, 437, 1362

Buote, D. A., Zappacosta, L., Fang, T., et al. 2009, ApJ, 695, 1351

Burke, C., Collins, C. A., Stott, J. P., \& Hilton, M. 2012, MNRAS, 425, 2058

Burke, C., Hilton, M., \& Collins, C. 2015, ArXiv e-prints

Busha, M. in prep., TBD.

Busha, M. T., Wechsler, R. H., Becker, M. R., Erickson, B., \& Evrard, A. E. 2013, in American Astronomical Society Meeting Abstracts, Vol. 221, American Astronomical Society Meeting Abstracts \#221, 341.07

Butcher, H., \& Oemler, Jr., A. 1978a, ApJ, 219, 18

-. 1978b, ApJ, 226, 559

-. 1984, ApJ, 285, 426

Byram, E. T., Chubb, T. A., \& Friedman, H. 1966, Science, 152, 66

Carlberg, R. G., Morris, S. L., Yee, H. K. C., \& Ellingson, E. 1997, ApJ, 479, L19

Chabrier, G. 2003, PASP, 115, 763

Chang, C., Busha, M. T., Wechsler, R. H., et al. 2014, ArXiv e-prints

-. 2015, ApJ, 801, 73 
Chilingarian, I., \& Zolotukhin, I. 2015, Science, 348, 418

Chilingarian, I. V., Melchior, A.-L., \& Zolotukhin, I. Y. 2010, MNRAS, 405, 1409

Chiu, I., Mohr, J., Mcdonald, M., et al. 2014, ArXiv e-prints

Colberg, J. M., Krughoff, K. S., \& Connolly, A. J. 2005, MNRAS, 359, 272

Colless, M., Dalton, G., Maddox, S., et al. 2001, MNRAS, 328, 1039

Collins, C. A., Stott, J. P., Hilton, M., et al. 2009, Nature, 458, 603

Conroy, C., \& Gunn, J. E. 2010, ApJ, 712, 833

Conroy, C., Gunn, J. E., \& White, M. 2009, ApJ, 699, 486

Conroy, C., Wechsler, R. H., \& Kravtsov, A. V. 2007, ApJ, 668, 826

Contini, E., De Lucia, G., Villalobos, Á., \& Borgani, S. 2014, MNRAS, 437, 3787

Cooray, A. 2006, MNRAS, 365, 842

Crawford, S. M., Bershady, M. A., \& Hoessel, J. G. 2009, ApJ, 690, 1158

Criminisi, A., Perez, P., \& Toyama, K. 2004, Image Processing, IEEE Transactions on, 13, 1200

Croton, D. J., Springel, V., White, S. D. M., et al. 2006, MNRAS, 367, 864

Cui, W., Murante, G., Monaco, P., et al. 2014, MNRAS, 437, 816

Cunha, C. E., Lima, M., Oyaizu, H., Frieman, J., \& Lin, H. 2009, MNRAS, 396, 2379

Dark Energy Survey Collaboration, Abbott, T., Abdalla, F. B., et al. 2016, ArXiv e-prints

Davé, R., Cen, R., Ostriker, J. P., et al. 2001, ApJ, 552, 473

Davis, M., Efstathiou, G., Frenk, C. S., \& White, S. D. M. 1985, ApJ, 292, 371

de Filippis, E., Paolillo, M., Longo, G., et al. 2011, MNRAS, 414, 2771

De Lucia, G., \& Blaizot, J. 2007, MNRAS, 375, 2

De Lucia, G., Poggianti, B. M., Aragón-Salamanca, A., et al. 2007, MNRAS, 374, 809

De Propris, R., Phillipps, S., \& Bremer, M. N. 2013, MNRAS, 434, 3469

DeMaio, T., Gonzalez, A., Zabludoff, A., Zaritsky, D., \& Bradac, M. 2015, ArXiv e-prints

Desai, S., Armstrong, R., Mohr, J. J., et al. 2012, ApJ, 757, 83

Diehl, H., Abbott, T., Annis, J., et al. 2014, in SPIE Astronomical Telescopes+ Instrumentation, International Society for Optics and Photonics, 91490V-91490V 
Diehl, H. T., Abbott, T. M. C., Annis, J., et al. 2014, in Society of Photo-Optical Instrumentation Engineers (SPIE) Conference Series, Vol. 9149, Society of Photo-Optical Instrumentation Engineers (SPIE) Conference Series, 0

Dietrich, J. P., Werner, N., Clowe, D., et al. 2012, Nature, 487, 202

Dietrich, J. P., Zhang, Y., Song, J., et al. 2014, MNRAS, 443, 1713

Diolaiti, E., Bendinelli, O., Bonaccini, D., et al. 2000, A\&AS, 147, 335

Donahue, M., Connor, T., Fogarty, K., et al. 2015, ArXiv e-prints

D’Onofrio, M., Bindoni, D., Fasano, G., et al. 2014, A\&A, 572, A87

Dressler, A. 1984, ARA\&A, 22, 185

Drinkwater, M. J., Gregg, M. D., Hilker, M., et al. 2003, Nature, 423, 519

Drinkwater, M. J., Jones, J. B., Gregg, M. D., \& Phillipps, S. 2000, pasa, 17, 227

Dubinski, J. 1998, ApJ, 502, 141

Eckmiller, H. J., Hudson, D. S., \& Reiprich, T. H. 2011, A\&A, 535, A105

Eisenhardt, P. R. M., Brodwin, M., Gonzalez, A. H., et al. 2008, ApJ, 684, 905

Eisenstein, D. J., Weinberg, D. H., Agol, E., et al. 2011, AJ, 142, 72

Ellingson, E., Lin, H., Yee, H. K. C., \& Carlberg, R. G. 2001, ApJ, 547, 609

Fabian, A. C. 1994, $A R A \& A, 32,277$

-. 2012, ARA\&A, 50, 455

Fang, T., Buote, D. A., Humphrey, P. J., et al. 2010, ApJ, 714, 1715

Federici, L., Fusi Pecci, F., Zavaroni, C., et al. 1983, Ap\&SS, 90, 405

Ferrari, C., Govoni, F., Schindler, S., Bykov, A. M., \& Rephaeli, Y. 2008, Space Sci. Rev., 134,93

Fioc, M., \& Rocca-Volmerange, B. 1997, A\&A, 326, 950

Flaugher, B., Diehl, H. T., Honscheid, K., et al. 2015a, AJ, 150, 150

-. 2015b, ArXiv e-prints

Foreman-Mackey, D., Hogg, D. W., Lang, D., \& Goodman, J. 2013, PASP, 125, 306

Fraser-McKelvie, A., Brown, M. J. I., \& Pimbblet, K. A. 2014, MNRAS, 444, L63

Fraser-McKelvie, A., Pimbblet, K. A., \& Lazendic, J. S. 2011, MNRAS, 415, 1961 
Galametz, A., Grazian, A., Fontana, A., et al. 2013, ApJS, 206, 10

Geller, M. J., \& Huchra, J. P. 1989, Science, 246, 897

Giallongo, E., Menci, N., Grazian, A., et al. 2014, ApJ, 781, 24

Gilbank, D. G., Gladders, M. D., Yee, H. K. C., \& Hsieh, B. C. 2011, AJ, 141, 94

Gilbank, D. G., Yee, H. K. C., Ellingson, E., et al. 2008, ApJ, 673, 742

Gladders, M. D., \& Yee, H. K. C. 2000, AJ, 120, 2148

Gobat, R., Daddi, E., Onodera, M., et al. 2011, A\&A, 526, A133

Goerdt, T., Moore, B., Kazantzidis, S., et al. 2008, MNRAS, 385, 2136

Gonzalez, A. H., Sivanandam, S., Zabludoff, A. I., \& Zaritsky, D. 2013, ApJ, 778, 14

Gonzalez, A. H., Zaritsky, D., \& Zabludoff, A. I. 2007, ApJ, 666, 147

Górski, K. M., Hivon, E., Banday, A. J., et al. 2005, ApJ, 622, 759

Graham, A. W., \& Driver, S. P. 2005, PASA, 22, 118

Groenewald, D. N., \& Loubser, S. I. 2014, MNRAS, 444, 808

Grützbauch, R., Bauer, A. E., Jørgensen, I., \& Varela, J. 2012, MNRAS, 423, 3652

Guennou, L., Adami, C., Da Rocha, C., et al. 2012, A\&A, 537, A64

Guo, Q., White, S., Angulo, R. E., et al. 2013, MNRAS, 428, 1351

Guo, Q., White, S., Boylan-Kolchin, M., et al. 2011, MNRAS, 413, 101

Hansen, S. M., Sheldon, E. S., Wechsler, R. H., \& Koester, B. P. 2009, ApJ, 699, 1333

Hao, J., McKay, T. A., Koester, B. P., et al. 2010, ApJS, 191, 254

Harrison, C. D., Miller, C. J., Richards, J. W., et al. 2012, ApJ, 752, 12

Hasselfield, M., Hilton, M., Marriage, T. A., et al. 2013, JCAP, 7, 8

Hausman, M. A., \& Ostriker, J. P. 1978, ApJ, 224, 320

Häussler, B., McIntosh, D. H., Barden, M., et al. 2007, ApJS, 172, 615

He, Y. Q., Xia, X. Y., Hao, C. N., et al. 2013, ApJ, 773, 37

Henriques, B. M. B., White, S. D. M., Lemson, G., et al. 2012, MNRAS, 421, 2904

Henry, J. P., Evrard, A. E., Hoekstra, H., Babul, A., \& Mahdavi, A. 2009, ApJ, 691, 1307

High, F. W., Stubbs, C. W., Rest, A., Stalder, B., \& Challis, P. 2009, AJ, 138, 110 
Hilker, M., Infante, L., Vieira, G., Kissler-Patig, M., \& Richtler, T. 1999, A\&AS, 134, 75

Hilton, M., Stanford, S. A., Stott, J. P., et al. 2009, ApJ, 697, 436

Hilton, M., Romer, A. K., Kay, S. T., et al. 2012, MNRAS, 424, 2086

Hoekstra, H., Donahue, M., Conselice, C. J., McNamara, B. R., \& Voit, G. M. 2011, ApJ, 726,48

Hoekstra, H., Mahdavi, A., Babul, A., \& Bildfell, C. 2012, MNRAS, 427, 1298

Hogg, D. W., Bovy, J., \& Lang, D. 2010, ArXiv e-prints

Hoyle, F., Rojas, R. R., Vogeley, M. S., \& Brinkmann, J. 2005, ApJ, 620, 618

Hoyle, F., Vogeley, M. S., \& Pan, D. 2012, MNRAS, 426, 3041

Hu, W., \& Kravtsov, A. V. 2003, ApJ, 584, 702

Huber, B., Tchernin, C., Eckert, D., et al. 2013, A\&A, 560, A64

Inagaki, T., Lin, Y.-T., Huang, H.-J., Hsieh, B.-C., \& Sugiyama, N. 2015, MNRAS, 446, 1107

Ivezić, Ž., Smith, J. A., Miknaitis, G., et al. 2007, AJ, 134, 973

Jõeveer, M., Einasto, J., \& Tago, E. 1978, MNRAS, 185, 357

Jaffé, Y. L., Aragón-Salamanca, A., De Lucia, G., et al. 2011, MNRAS, 410, 280

Janz, J., Forbes, D. A., Norris, M. A., et al. 2015, MNRAS, 449, 1716

Jarvis, J. F., \& Tyson, J. A. 1981, AJ, 86, 476

Jauzac, M., Jullo, E., Kneib, J.-P., et al. 2012, MNRAS, 426, 3369

Jing, Y. P., Mo, H. J., \& Börner, G. 1998, ApJ, 494, 1

Johnston, D. E., Sheldon, E. S., Wechsler, R. H., et al. 2007, ArXiv e-prints

Kauffmann, G. 1995, MNRAS, 274, 153

Kelly, P. L., von der Linden, A., Applegate, D. E., et al. 2014, MNRAS, 439, 28

Kettula, K., Finoguenov, A., Massey, R., et al. 2013, ApJ, 778, 74

Klypin, A. A., \& Shandarin, S. F. 1983, MNRAS, 204, 891

Koda, J., Yagi, M., Yamanoi, H., \& Komiyama, Y. 2015, ApJ, 807, L2

Koester, B. P., McKay, T. A., Annis, J., et al. 2007a, ApJ, 660, 239

-. 2007b, ApJ, 660, 221 
Kravtsov, A., Vikhlinin, A., \& Meshscheryakov, A. 2014, ArXiv e-prints

Kravtsov, A. V., Vikhlinin, A., \& Nagai, D. 2006, ApJ, 650, 128

Krick, J. E., \& Bernstein, R. A. 2007, AJ, 134, 466

Krick, J. E., Bernstein, R. A., \& Pimbblet, K. A. 2006, AJ, 131, 168

Kron, R. G. 1980, ApJS, 43, 305

Kroupa, P. 2001, MNRAS, 322, 231

Kurk, J., Cimatti, A., Zamorani, G., et al. 2009, A\&A, 504, 331

Lan, T.-W., Ménard, B., \& Mo, H. 2015, ArXiv e-prints

Lang, D., Hogg, D. W., \& Schlegel, D. J. 2014, ArXiv e-prints

Laporte, C. F. P., White, S. D. M., Naab, T., \& Gao, L. 2013, MNRAS, 435, 901

Lauer, T. R., Faber, S. M., Richstone, D., et al. 2007, ApJ, 662, 808

Leauthaud, A., Tinker, J., Bundy, K., et al. 2012, ApJ, 744, 159

Li, I. H., Yee, H. K. C., \& Ellingson, E. 2009, ApJ, 698, 83

Li, I. H., Yee, H. K. C., Hsieh, B. C., \& Gladders, M. 2012, ApJ, 749, 150

Li, Y., \& Bryan, G. L. 2014a, ApJ, 789, 54

-. 2014b, ApJ, 789, 153

Lidman, C., Suherli, J., Muzzin, A., et al. 2012, MNRAS, 427, 550

Lin, H., Soares-Santos, M., Diehl, H., \& Dark Energy Survey Collaboration. 2013a, in American Astronomical Society Meeting Abstracts, Vol. 221, American Astronomical Society Meeting Abstracts \#221, 352.26

Lin, H., Kuropatkin, N., Wechsler, R., et al. 2010, in Bulletin of the American Astronomical Society, Vol. 42, American Astronomical Society Meeting Abstracts \#215, 470.07

Lin, Y.-T., Brodwin, M., Gonzalez, A. H., et al. 2013b, ApJ, 771, 61

Lin, Y.-T., \& Mohr, J. J. 2004, ApJ, 617, 879

Lindeberg, T. 1993, Scale-space theory in computer vision (Springer)

Liu, C., Peng, E. W., Côté, P., et al. 2015, ApJ, 812, 34

Liu, F. S., Mao, S., \& Meng, X. M. 2012, MNRAS, 423, 422

Liu, F. S., Guo, Y., Koo, D. C., et al. 2013, ApJ, 769, 147 
Lloyd-Davies, E. J., Romer, A. K., Mehrtens, N., et al. 2011, MNRAS, 418, 14

Lu, Z., Mo, H. J., Lu, Y., et al. 2014, MNRAS, 439, 1294

Lupton, R., Gunn, J. E., Ivezić, Z., Knapp, G. R., \& Kent, S. 2001, in Astronomical Society of the Pacific Conference Series, Vol. 238, Astronomical Data Analysis Software and Systems X, ed. F. R. Harnden, Jr., F. A. Primini, \& H. E. Payne, 269

MacDonald, E. C., Allen, P., Dalton, G., et al. 2004, MNRAS, 352, 1255

Maddox, S. J., Efstathiou, G., Sutherland, W. J., \& Loveday, J. 1990, MNRAS, 243, 692

Mahdavi, A., Hoekstra, H., Babul, A., et al. 2013, ApJ, 767, 116

Malarecki, J. M., Staveley-Smith, L., Saripalli, L., et al. 2013, MNRAS

Mancone, C. L., \& Gonzalez, A. H. 2012, PASP, 124, 606

Mandelbaum, R., Seljak, U., Kauffmann, G., Hirata, C. M., \& Brinkmann, J. 2006, MN$R A S, 368,715$

Mandelbaum, R., Hirata, C. M., Seljak, U., et al. 2005, MNRAS, 361, 1287

Mantz, A., Allen, S. W., Ebeling, H., Rapetti, D., \& Drlica-Wagner, A. 2010, MNRAS, 406, 1773

Maraston, C. 2005, MNRAS, 362, 799

Margoniner, V. E., de Carvalho, R. R., Gal, R. R., \& Djorgovski, S. G. 2001, ApJ, 548, L143

Markwardt, C. B. 2009, in Astronomical Society of the Pacific Conference Series, Vol. 411, Astronomical Data Analysis Software and Systems XVIII, ed. D. A. Bohlender, D. Durand, \& P. Dowler, 251

Marriage, T. A., Acquaviva, V., Ade, P. A. R., et al. 2011, ApJ, 737, 61

Martinet, N., Durret, F., Guennou, L., et al. 2015, A\&A, 575, A116

Martizzi, D., Jimmy, Teyssier, R., \& Moore, B. 2014, MNRAS, 443, 1500

Martizzi, D., Teyssier, R., \& Moore, B. 2012, MNRAS, 420, 2859

Matthews, T. A., Morgan, W. W., \& Schmidt, M. 1964, ApJ, 140, 35

Maughan, B. J., Giles, P. A., Randall, S. W., Jones, C., \& Forman, W. R. 2012, MNRAS, 421,1583

McDonald, M., Bayliss, M., Benson, B. A., et al. 2012, Nature, 488, 349

McGee, S. L., Balogh, M. L., Bower, R. G., Font, A. S., \& McCarthy, I. G. 2009, MNRAS, 400, 937 
Meece, G., O’Shea, B., \& Voit, M. 2015, ArXiv e-prints

Meert, A., Vikram, V., \& Bernardi, M. 2015, MNRAS, 446, 3943

Mehrtens, N., Romer, A. K., Hilton, M., et al. 2012, MNRAS, 423, 1024

Mei, S., Holden, B. P., Blakeslee, J. P., et al. 2006, ApJ, 644, 759

Meyer, F. 1992, in Image Processing and its Applications, 1992., International Conference on, 303-306

Mieske, S., Frank, M. J., Baumgardt, H., et al. 2013, A\&A, 558, A14

Mihos, J. C., Durrell, P. R., Ferrarese, L., et al. 2015, ApJ, 809, L21

Miller, C. J., Rooney, P., \& The DES collaboration. in prep., TBD.

Miller, C. J., Nichol, R. C., Reichart, D., et al. 2005, AJ, 130, 968

Mitchell, P. D., Lacey, C. G., Baugh, C. M., \& Cole, S. 2013, MNRAS, 435, 87

Mohr, J. J., Armstrong, R., Bertin, E., et al. 2012, in Society of Photo-Optical Instrumentation Engineers (SPIE) Conference Series, Vol. 8451, Society of Photo-Optical Instrumentation Engineers (SPIE) Conference Series

Monaco, P., Murante, G., Borgani, S., \& Fontanot, F. 2006, ApJ, 652, L89

Montero-Dorta, A. D., \& Prada, F. 2009, MNRAS, 399, 1106

Montes, M., \& Trujillo, I. 2014, ApJ, 794, 137

Moster, B. P., Naab, T., \& White, S. D. M. 2013, MNRAS, 428, 3121

Moster, B. P., Somerville, R. S., Maulbetsch, C., et al. 2010, ApJ, 710, 903

Muñoz, R. P., Eigenthaler, P., Puzia, T. H., et al. 2015, ApJ, 813, L15

Mullis, C. R., Rosati, P., Lamer, G., et al. 2005, ApJ, 623, L85

Muzzin, A., Wilson, G., Demarco, R., et al. 2013, ApJ, 767, 39

Nagai, D., Vikhlinin, A., \& Kravtsov, A. V. 2007, ApJ, 655, 98

Norris, M. A., Escudero, C. G., Faifer, F. R., et al. 2015, MNRAS, 451, 3615

Norris, M. A., Kannappan, S. J., Forbes, D. A., et al. 2014, MNRAS, 443, 1151

Oliva-Altamirano, P., Brough, S., Lidman, C., et al. 2014, MNRAS, 440, 762

Ostriker, J. P., \& Tremaine, S. D. 1975, ApJ, 202, L113

Papadopoulos, A., D’Andrea, C. B., Sullivan, M., et al. 2015, MNRAS, 449, 1215 
Papovich, C., Finkelstein, S. L., Ferguson, H. C., Lotz, J. M., \& Giavalisco, M. 2011, MNRAS, 412, 1123

Papovich, C., Momcheva, I., Willmer, C. N. A., et al. 2010, ApJ, 716, 1503

Patel, S. G., van Dokkum, P. G., Franx, M., et al. 2013, ApJ, 766, 15

Paudel, S., Lisker, T., \& Janz, J. 2010, ApJ, 724, L64

Peacock, J. A., \& Smith, R. E. 2000, MNRAS, 318, 1144

Peng, C. Y., Ho, L. C., Impey, C. D., \& Rix, H.-W. 2002, AJ, 124, 266

-. 2010a, AJ, 139, 2097

Peng, Y., Maiolino, R., \& Cochrane, R. 2015, Nature, 521, 192

Peng, Y.-j., Lilly, S. J., Kovač, K., et al. 2010b, ApJ, 721, 193

Petrosian, V. 1976, ApJ, 209, L1

Pfeffer, J., \& Baumgardt, H. 2013, MNRAS, 433, 1997

Phillipps, S., Drinkwater, M. J., Gregg, M. D., \& Jones, J. B. 2001, ApJ, 560, 201

Pike, S. R., Kay, S. T., Newton, R. D. A., Thomas, P. A., \& Jenkins, A. 2014, MNRAS, 445, 1774

Pimbblet, K. A., Drinkwater, M. J., \& Hawkrigg, M. C. 2004, MNRAS, 354, L61

Planck Collaboration, Ade, P. A. R., Aghanim, N., et al. 2014, A\&A, 571, A29

-. 2015, ArXiv e-prints

Popesso, P., Biviano, A., Böhringer, H., \& Romaniello, M. 2006, A\&A, 445, 29

Popesso, P., Böhringer, H., Romaniello, M., \& Voges, W. 2005, A\&A, 433, 415

Porter, S. C., Raychaudhury, S., Pimbblet, K. A., \& Drinkwater, M. J. 2008, MNRAS, 388, 1152

Pratt, G. W., Croston, J. H., Arnaud, M., \& Böhringer, H. 2009, A\&A, 498, 361

Presotto, V., Girardi, M., Nonino, M., et al. 2014, A\&A, 565, A126

Puchwein, E., Springel, V., Sijacki, D., \& Dolag, K. 2010, MNRAS, 406, 936

Ragone-Figueroa, C., Granato, G. L., Murante, G., Borgani, S., \& Cui, W. 2013, MNRAS, 436,1750

Rasia, E., Borgani, S., Ettori, S., Mazzotta, P., \& Meneghetti, M. 2013, ApJ, 776, 39

Rasia, E., Meneghetti, M., Martino, R., et al. 2012, New Journal of Physics, 14, 055018 
Reichardt, C. L., Stalder, B., Bleem, L. E., et al. 2013, ApJ, 763, 127

Rettura, A., Martinez-Manso, J., Stern, D., et al. 2014, ApJ, 797, 109

Richstone, D. O., \& Malumuth, E. M. 1983, ApJ, 268, 30

Roche, N., Bernardi, M., \& Hyde, J. 2009, MNRAS, 398, 1549

Rudick, C. S. 2010, PhD thesis, Case Western Reserve University

Rudick, C. S., Mihos, J. C., \& McBride, C. K. 2011, ApJ, 732, 48

Rudnick, G., von der Linden, A., Pelló, R., et al. 2009, ApJ, 700, 1559

Ruszkowski, M., \& Springel, V. 2009, ApJ, 696, 1094

Rykoff, E., Bechtol, R., \& DES Collaboration. in prep., TBD

Rykoff, E. S., Koester, B. P., Rozo, E., et al. 2012, ApJ, 746, 178

Rykoff, E. S., Rozo, E., Busha, M. T., et al. 2013, ArXiv e-prints

-. 2014, ApJ, 785, 104

Sánchez, C., Carrasco Kind, M., Lin, H., et al. 2014, MNRAS, 445, 1482

Sánchez, E. 2010, Journal of Physics Conference Series, 259, 012080

Santos, J. S., Fassbender, R., Nastasi, A., et al. 2011, A\&A, 531, L15

Sarazin, C. L. 1988, X-ray emission from clusters of galaxies

Savage, R. S., \& Oliver, S. 2007, ApJ, 661, 1339

Schechter, P. 1976, ApJ, 203, 297

Seljak, U. 2000, MNRAS, 318, 203

Seth, A. C., van den Bosch, R., Mieske, S., et al. 2014, Nature, 513, 398

Sethian, J. A. 1996, Proceedings of the National Academy of Sciences, 93, 1591

Shankar, F., Buchan, S., Rettura, A., et al. 2015, ArXiv e-prints

Sheldon, E. S., Johnston, D. E., Frieman, J. A., et al. 2004, AJ, 127, 2544

Sheldon, E. S., Johnston, D. E., Scranton, R., et al. 2009a, ApJ, 703, 2217

Sheldon, E. S., Johnston, D. E., Masjedi, M., et al. 2009b, ApJ, 703, 2232

Simard, L., Willmer, C. N. A., Vogt, N. P., et al. 2002, The Astrophysical Journal Supplement Series, 142, 1 
Skrutskie, M. F., Cutri, R. M., Stiening, R., et al. 2006, AJ, 131, 1163

Smail, I., Edge, A. C., Ellis, R. S., \& Blandford, R. D. 1998, MNRAS, 293, 124

Soares-Santos, M., de Carvalho, R. R., Annis, J., et al. 2011, ApJ, 727, 45

Springel, V., White, S. D. M., Jenkins, A., et al. 2005, Nature, 435, 629

Stanford, S. A., Eisenhardt, P. R., Brodwin, M., et al. 2005, ApJ, 634, L129

Staniszewski, Z., Ade, P. A. R., Aird, K. A., et al. 2009, ApJ, 701, 32

Stetson, P. B. 1987, PASP, 99, 191

Stott, J. P., Collins, C. A., Burke, C., Hamilton-Morris, V., \& Smith, G. P. 2011, MNRAS, 414,445

Stott, J. P., Smail, I., Edge, A. C., et al. 2007, ApJ, 661, 95

Stott, J. P., Collins, C. A., Sahlén, M., et al. 2010, ApJ, 718, 23

Stott, J. P., Hickox, R. C., Edge, A. C., et al. 2012, MNRAS, 422, 2213

Stoughton, C., Lupton, R. H., Bernardi, M., et al. 2002, AJ, 123, 485

Suchyta, E., Huff, E. M., Aleksić, J., et al. 2015, ArXiv e-prints

Sun, M., Voit, G. M., Donahue, M., et al. 2009, ApJ, 693, 1142

Sunyaev, R. A., \& Zeldovich, Y. B. 1969, Nature, 223, 721

—. 1972, Comments on Astrophysics and Space Physics, 4, 173

Tanaka, M., Finoguenov, A., Mirkazemi, M., et al. 2013, PASJ, 65, 17

Telea, A. 2004, Journal of Graphics Tools, 9, 23

The Fermi-LAT Collaboration, \& Rephaeli, Y. 2015, ArXiv e-prints

Toledo, I., Melnick, J., Selman, F., et al. 2011, MNRAS, 414, 602

Tollerud, E. J., Bullock, J. S., Graves, G. J., \& Wolf, J. 2011, ApJ, 726, 108

Tonini, C., Bernyk, M., Croton, D., Maraston, C., \& Thomas, D. 2012, ApJ, 759, 43

Tremaine, S. D., \& Richstone, D. O. 1977, ApJ, 212, 311

Trentham, N., \& Hodgkin, S. 2002, MNRAS, 333, 423

van den Bosch, F. C., Yang, X., Mo, H. J., et al. 2007, MNRAS, 376, 841

van der Burg, R. F. J., Muzzin, A., Hoekstra, H., et al. 2014, A\&A, 561, A79 
van Dokkum, P. G., Abraham, R., Merritt, A., et al. 2015a, ApJ, 798, L45

van Dokkum, P. G., Whitaker, K. E., Brammer, G., et al. 2010, ApJ, 709, 1018

van Dokkum, P. G., Romanowsky, A. J., Abraham, R., et al. 2015b, ApJ, 804, L26

Viana, P. T. P., Mehrtens, N., Harrison, C. D., et al. 2013, Astronomische Nachrichten, 334, 462

Vikhlinin, A., Burenin, R. A., Ebeling, H., et al. 2009, ApJ, 692, 1033

Voit, G. M. 2005, Reviews of Modern Physics, 77, 207

Voit, G. M., Donahue, M., Bryan, G. L., \& McDonald, M. 2015, Nature, 519, 203

von der Linden, A., Best, P. N., Kauffmann, G., \& White, S. D. M. 2007, MNRAS, 379, 867

Webb, T. M. A., Muzzin, A., Noble, A., et al. 2015, ApJ, 814, 96

Wechsler, R. in prep., TBD.

Weinberg, D. H., Mortonson, M. J., Eisenstein, D. J., et al. 2013, Phys. Rep., 530, 87

Wen, Z. L., \& Han, J. L. 2015, MNRAS, 448, 2

Werner, N., Finoguenov, A., Kaastra, J. S., et al. 2008, A\&A, 482, L29

Whiley, I. M., Aragón-Salamanca, A., De Lucia, G., et al. 2008, MNRAS, 387, 1253

White, S. D. M. 1976, MNRAS, 174, 19

Williams, M. J., Bureau, M., \& Cappellari, M. 2010, MNRAS, 409, 1330

Yamanoi, H., Komiyama, Y., Yagi, M., et al. 2012, AJ, 144, 40

Yang, X., Mo, H. J., \& van den Bosch, F. C. 2003, MNRAS, 339, 1057

-. 2008, ApJ, 676, 248

—. 2009, ApJ, 695, 900

Yasuda, N., Fukugita, M., Narayanan, V. K., et al. 2001, AJ, 122, 1104

Yee, H. K. C. 1991, PASP, 103, 396

York, D. G., Adelman, J., Anderson, Jr., J. E., et al. 2000, AJ, 120, 1579

Zarattini, S., Aguerri, J. A. L., Sánchez-Janssen, R., et al. 2015, A\&A, 581, A16

Zehavi, I., Zheng, Z., Weinberg, D. H., et al. 2011, ApJ, 736, 59

Zeldovich, Y. B. 1970, A\&A, 5, 84 
Zhang, H.-X., Peng, E. W., Côté, P., et al. 2015a, ApJ, 802, 30

Zhang, Y., Dietrich, J. P., McKay, T. A., Sheldon, E. S., \& Nguyen, A. T. Q. 2013, ApJ, 773,115

Zhang, Y., McKay, T. A., Bertin, E., et al. 2015b, PASP, 127, 1183

Zhang, Y., Miller, C., Mckay, T., et al. 2015c, ArXiv e-prints

Zheng, Z., Coil, A. L., \& Zehavi, I. 2007, ApJ, 667, 760

Zibetti, S., White, S. D. M., Schneider, D. P., \& Brinkmann, J. 2005, MNRAS, 358, 949 